\title{
Affektregime der Primatologie
}

\section{1 "primatology red in tooth, claw, and ideology" ${ }^{1}$ ? - Strategien der frühen Primatologie}

Affekte im Feld lassen sich in den Forschungsmemoiren der Primatologie, wie in den letzten beiden Teilen diskutiert, auf mindestens zweierlei Ebenen untersuchen: Es lässt sich erstens nach der Qualität von Affekten in der Forschung fragen und der epistemologische Wert von Emotionen in Forschungsprozessen aus dem Berichten über die daran beteiligten Prozesse herausarbeiten. Zweitens kann anhand der Darstellung von herausfordernden Erfahrungen aus dem Forschungskontext der affektpoetologische Zusammenhang von Form und Emotionalität beleuchtet werden. Die Thematisierbarkeit von Affektivität in der Forschung und die affektive Darstellung von Forschungserfahrungen sind jedoch beide drittens abhängig von dem, was zeitgenössisch denk- und darstellbar ist. Wie im Folgenden aufzuzeigen sein wird, sind die primatologischen Forschungsmemoiren nicht unabhängig von ihren jeweiligen gesellschaftlichen, kulturellen, politischen und/ oder wissenschaftlichen Kontexten zu betrachten, denn diese geben in Form verschiedener Regime vor, ob, wie und wann von Emotionalität berichtet werden kann.

Zunächst soll zu diesem Zweck ein Blick auf die Forschung an Affen und ihre Paradigmen in der ersten Hälfte des 20. Jahrhunderts geworfen werden. Wie die ,Causa Anthropomorphismus“ (Abschn. 4.1.1) bei Wolfgang Köhler und Solly Zuckerman zeigt, muss sich bereits die experimentell arbeitende, frühe Primatologie mit dem Vorwurf der affektiven Unwissenschaftlichkeit auseinandersetzen. Wie die Autoren dabei im Einzelnen damit und mit dem schwierigen Stand anekdotischen Wissens in dieser wissenschaftlichen Episteme strategisch im

${ }^{1}$ So Allison Jolly über Solly Zuckermans Forschung; Jolly: The Bad Old Days of Primatology, S. 74. 
Text umgehen, soll anhand der Abschn. 4.1.2 und 4.1.3 genauer untersucht werden, bevor Affektregime, Gefühlsregeln und wissenschaftliche Paradigmen als Determinatoren für die Äußer- und Darstellbarkeit von Emotionalität im primatologischen Text näher erläutert werden (Abschn. 4.1.4).

\subsubsection{Die ,Causa Anhropomorphismus‘}

In der ersten Hälfte des 20. Jahrhunderts manifestierte sich in verschiedenen wissenschaftlichen Bereichen ein Interesse an der nicht mehr nur anatomischen (an toten Exemplaren vollzogenen) sondern nun auch empirischen Forschung an (lebenden) Affen. Dies sollte die vielfältige disziplinäre Ausrichtung des späteren Feldes der Primatologie entscheidend und unterschiedlich prägen. Neben dem Psychologen und Mitbegründer der Tierpsychologie Robert Yerkes und seinem Schüler Clarence Ray Carpenter, deren wissenschaftliche Netzwerke Haraway bereits untersucht hat, beginnen auch der Psychologe Wolfgang Köhler ${ }^{2}$ als Leiter der Anthropoidenstation der Preußischen Akademie der Wissenschaften auf Teneriffa ab 1914 und um 1930 der britische Zoologe Solly Zuckerman ${ }^{3}$ als Anatom der Zoological Society of London, sich mit Affen zu beschäftigen. ${ }^{4}$ So unterschiedlich ihre Ansätze und Forschungsinteressen sind - hier die Fähigkeiten zur kognitiven Problemlösung im Kontext der von Köhler mitbegründeten neuen Gestaltpsychologie, dort physiologisch bestimmte Reproduktivität als fundamentales Konzept primatischer Sozialstruktur bei Zuckerman -, so verschieden fallen

\footnotetext{
${ }^{2}$ Wolfgang Köhler (1887-1967) promovierte 1909 bei Carl Stumpf am Psychologischen Institut der Friedrich-Wilhelms-Universität in Berlin. Gemeinsam mit den anderen Stumpf-Schülern Max Wertheimer und Kurt Koffka begründete er die Berliner Schule der Gestaltpsychologie, die er ab 1921 als Leiter des Psychologischen Instituts an der Berliner Universität gegen den Assoziationismus und den Behaviorismus durchsetzte und die nach seinem Rückzug von der nationalsozialistisch gleichgeschalteten Friedrich-Wilhelms-Universität ab 1935 eine einflussreiche Fortsetzung in den USA fand. Vgl. Pratt, Carroll C.: Wolfgang Köhler, 1887-1967 - Einführung. In: Wolfgang Köhler: Die Aufgabe der Gestaltpsychologie. Berlin/New York 1971 (engl. 1969), S. 3-21, hier S. 5 ff.; Jaeger, Siegfried: Einleitung des Herausgebers. In: Siegfried Jaeger (Hg.): Briefe von Wolfgang Köhler an Hans Geitel 1807-1920. Passau 1988, S. 7-13.

${ }^{3}$ In Kapstadt geboren studierte Solly Zuckerman (1904-1993) in der damaligen Union of South Africa Medizin. Hier führte er erste Beobachtungen an wilden Pavianen durch. Vermittelt durch den britischen Anatomen Elliot Smith, dessen Aufmerksamkeit er durch erste anatomische Arbeiten erlangte, wurde Zuckerman in London, wo er seine Studien fortsetzte, Mitglied der Zoographical Society. Protegiert von Smith und dem britischen Evolutionsbiologen Julian Huxley erlangte Zuckerman mit 23 Jahren eine Anstellung als Anatomical Research Fellow in der Zoological Society of London. Vgl. Donovan, Bernard: Zuckerman: Scientist Extraordinary. Bristol 2005, S. 1-9.

${ }^{4}$ Haraway rekonstruiert, dass Yerkes und Carpenter von Zuckermans Ansatz und Veröffentlichung beeindruckt waren. Zwar hegte Yerkes vereinzelte Zweifel an der Generalisierbarkeit von Zuckermans Gedanken, Carpenter nahm sie jedoch in seine Forschungspraxis auf. Vgl. Haraway: Primate Visions, S. 59-83, 84-111, besonders 90 f.
} 
auch die Texte der beiden Autoren aus. Sie zeigen jedoch trotz diverser Unterschiede und den 15 historisch nicht unwichtigen Jahren, die zwischen den Publikationen liegen, beide eine deutliche Auseinandersetzung mit der Gefahr, des Anthropomorphismus bezichtigt zu werden.

Köhler weist in den Intelligenzprüfungen an Anthropoiden ${ }^{5}$ bestimmte Verhaltensbeobachtungen - „Sultan zieht eine Kiste unter das Ziel, steigt aber nach einem Blick, der deutlich die Distanz mißt, nicht hinauf“ (33, Hervorheb. im Original) - als ,kein ,Anthropomorphismus““ (33) aus und erläutert in einer Fußnote die Verwendung der Formulierung, indem er sie als Teil der Verhaltensökologie des Schimpansen einordnet:

Jeden Tag kann man sehen, daß ein Schimpanse, der in großer Höhe zum Sprung über weite Distanzen ansetzt, vorher genau so wie hier mit dem Blick hinüber und herüber fährt; als Baumtier, das mitunter gewaltig springt, $т и ß$ er ja so schätzen können; es wäre also ganz unangebracht ängstlich, wollte man jene Wendung beanstanden. (33, Hervorheb. im Original)

Später im Text bittet er ,noch einmal dringend, nicht von ,Anthropomorphismus', von ,Hineinlegen in das Tier' u. dgl. zu sprechen, wo nicht der mindeste Grund für derartige Vorwürfe ist" (73). In Bezug auf die Feststellung, das Schimpansenmännchen Sultan trabe ,durchaus nicht vom Ziel fort in der freien, unbekümmerten Art, die man in neutralen Momenten an ihm und den andern sieht, sondern es geht fort wie jemand, der eine Aufgabe hat“ (73), sieht er in den „,beiden hier einander gegenübergestellten Gesamteindrücken“ „nichts in den Schimpansen Hineingelegtes“, sondern etwas ,zur elementaren Phänomenologie des Schimpansenverhaltens“ (73) Gehörendes. Mit den Formulierungen sei nichts über das „Bewußtsein des Tieres“ (73) gesagt, sondern nur über sein Verhalten.

Solly Zuckerman ist es wichtig, gleich in seiner Preface zu The Social Life of Monkeys and Apes eine wissenschaftsethische Erklärung anzubieten:

My own observations have been made without, I hope, anthropomorphic bias, and my interpretations have been weighted with no more than the insistence of the facts themselves. I have approached the subject from the deterministic point of view of the physiologist, treating overt behaviour as the result or expression of physiological events which have been made obvious through experimental analysis. (xix f.)

Anthropomorphismen sind seiner Ansicht nach nicht nur deshalb zu vermeiden, weil ,anthropomorphic preoccupations do not help the critical development of knowledge“ (8), sondern vor allem auch, weil er sie mit einem ,emotional bias“ (173) assoziiert. In diesem Zusammenhang gibt er nicht nur Götter an, die

\footnotetext{
${ }^{5}$ In der Folge wird aus der zweiten, durchgesehenen Auflage zitiert, die 1921 unter dem Titel Intelligenzprüfungen an Menschenaffen erschienen ist, und die im Vorwort als ,,in allem Wesentlichen unveränderter Neudruck“ (o. S.) der vergriffenen Erstauflage bezeichnet wird: Köhler, Wolfgang: Intelligenzprüfungen an Menschenaffen. Berlin ${ }^{2} 1921$. Im Folgenden, wenn zur Unterscheidung nötig, als $I M$ geführt; Seitenangaben im Text.
} 
aus dem Himmel verbannt werden mussten, damit astronomische Kenntnisse gewonnen werden konnten, sondern verweist auch darauf, dass ,[t]he emotions of the moth had to be disregarded before the nature of its reactions to light stimuli could be scientifically explained“" $8 \mathrm{f}$.). Diese Verschiebung von der unterstellten Emotionalität im anthropomorphisierenden Denken zur zu ignorierenden Emotionalität des Gegenstandes wird an späterer Stelle noch zu diskutieren sein (siehe Abschn. 4.2.1). Bei Solly Zuckerman verrät sie seine Verankerung in jenem von Strum und Fedigan identifizierten ,Vorstadium' primatologischer Forschung, deren Konzentration auf soziales Verhalten unter dem Zeichen eines strukturalistischen Funktionalismus steht. ${ }^{6}$ Der erste Teil von Zuckermans Beispiel - der götterlose Himmel der Astronomie - ist jedoch im Kontext neuerer Betrachtungen zum anthropomorphisierenden Denken kaum zufällig. Verweisen doch Lorraine Daston und Gregg Mitman auf das wesentliche Problem des Anthropomorphismus-Vorwurfs, wie er seit dem frühen 19. Jahrhundert auftaucht:

\begin{abstract}
,Anthropomorphism" is the word used to describe the belief that animals are essentially like humans, and it is usually applied as a form of reproach, both intellectual and moral. Originally, the word referred to the attribution of human form to gods, forbidden by several religions as blasphemous. Something of the religious taboo still clings to secular, modern instances of anthropomorphism, even if it is animals rather than divinities that are being humanized. ${ }^{7}$
\end{abstract}

Sandra Mitchell charakterisiert anthropomorphisierende Verfahren als ,claims about the similarity of nonhuman objects or beings to humans and the centrality of human concepts and abilities to classify behavior across ontological categories" ${ }^{* 8}$ und sieht sie als per se in einer Traditionslinie mit den Erkenntnissen der Evolutionsbiologie und der frühen Verhaltensforschung stehend. Dass diese Tradition mit dem Vorwurf des Sentimentalismus (bei Zuckerman eben: „emotional bias“) und der Naivität belegt wird, trägt auch eine moralische Implikation. ${ }^{9}$ Sie erwächst aus jenem in der Begriffsgeschichte des Anthropomorphismus liegenden Vermenschlichungsverbot für die Götter, wie es Daston und Mitman anführen, speist sich aus der für eine Rechtfertigung menschlichen Verhaltens gegenüber Tieren entscheidenden

\footnotetext{
${ }^{6}$ Siehe Strum/Fedigan: Changing Views of Primate Society, S. 9.

${ }^{7}$ Daston, Lorraine/Mitman, Gregg: Introduction: The How and Why of Thinking With Animals. In: Dies. (Hg.): Thinking With Animals. New Perspectives on Anthropomorphism. New York 2005, S. 1-13, hier S. 2.

${ }^{8}$ Mitchell, Sandra D.: Anthropomorphism and Cross-Species Modeling. In: Lorraine Daston/Gregg Mitman (Hg.): Thinking With Animals. New Perspectives on Anthropomorphism. New York 2005, S. $100-118$.

${ }^{9}$ Vgl. Sober, Elliott: Comparative Psychology Meets Evolutionary Biology. Morgan's Canon and Cladistic Parsimony. In: Lorraine Daston/Gregg Mitman (Hg.): Thinking With Animals. New Perspectives on Anthropomorphism. New York 2005, S. 85-99, hier S. 85.
} 
Konstruktion einer anthropologischen Differenz, ${ }^{10}$ aber auch aus der Assoziation von Anthropomorphismus mit dem ebenfalls kritisierten anekdotischem Material in der Verhaltensforschung. Dieses ankedotische Material wiederum wird als ,,a web of romance“ $(S L, 2)$, ,endless flights into the realms of imagination“ ( $S L$, $11)$, „difficulties of separating the fanciful from the true“ $(S L, 13)$, „stories whose origins are lost in the past" $(S L, 13)$ und ,lack of any exact knowledge“ $(S L, 13)$ abgelehnt, wie Zuckerman in seinen Text einarbeitet (vgl. auch Abschn. 2.2.2). Damit wird Anthropomorphismus bei Zuckerman über seine Assoziation mit dem anekdotischen Erzählen mit dem Bereich der Fiktion verbunden, und sein vermeintliches Fehlen zum Attribut des ,Realismus', dem sich moderne Wissenschaftler/ innen verschreiben wollen. Im Abwehrreflex fällt die wissenschaftliche Anthropomorphismus-Kritik jedoch schnell in ein erkenntnistheoretisch problematisches ,Anthropodenial'. Elliott Sober nutzt diesen Begriff von Frans de Waal, um den „error of attributing human mental characteristics to nonhuman organisms“, ${ }^{11}$ als welcher der Anthropomorphismus meist definiert wird, mit dem ,mistakenly refusing to attribute human mental characteristics to nonhuman organisms"12 $\mathrm{zu}$ paaren. Dabei werden diese beiden Denkmuster in wissenschaftlichen Gemeinschaften bezeichnenderweise mit Persönlichkeitsmerkmalen assoziiert: Der ,type-1 error of mistaken anthropomorphism is often taken to reflect a kind of tenderheartedness“, während der ,type-2 error of mistaken anthropodenial is supposed to reveal a kind of tough-mindedness “ ${ }^{13}$ wie Sober es formuliert. Dass der Anthropomorphismus-Verdacht als Vorwurf der Unwissenschaftlichkeit noch immer virulent ist, und für sein Gegenstück kaum Bewusstsein kreiert wird, liegt für Sober an einem ,,more general pattern in scientific culture in which tough-mindedness is valued."14 Diese subtile, zugleich wertende und polarisierende Emotionalisierung wissenschaftlicher

\footnotetext{
${ }^{10}$ Vgl. das Einleitungskapitel der vorliegenden Studie. Auf die ethische Problematik der anthropologischen Differenz verweisen ebenfalls Daston/Mitman: Introduction, S. 4 f. Sie vernachlässigen jedoch m. E. dass der Anthropomorphismus- als Blasphemievorwurf im Kontext der modernen Wissenschaft den Gegenstand gewechselt hat: Es geht nicht mehr darum, die Vermenschlichung als Erniedrigung der Götter zu betrachten, sondern in der evolutionären Kontinuität von Verhalten eine Erniedrigung des Menschen zu wittern.

${ }^{11}$ Sober: Comparative Psychology, S. 85.

${ }^{12}$ Sober: Comparative Psychology, S. 86.

${ }^{13}$ Sober: Comparative Psychology, S. 86, Hervorheb. MS.

${ }^{14}$ Sober: Comparative Psychology, S. 86. Als beispielhaft für den Kriegszug gegen Anthropomorphismus siehe Kennedy, John S.: The New Anthropomorphism. Cambridge u. a. 1992. Siehe aber zur Diskussion um den Wert, die Geschichte und die Unausweichlichkeit anthropomorphisierenden Denkens neben den bereits angeführten Überlegungen Latour, Bruno: Agency at the Time of the Anthropocene. In: New Literary History 45/1 (2014), S. 1-18; Wild: Anthropomorphismus. Marianne Sommer wies bereits 2000 in ihrer diskurslinguistischen Analyse von National-Geographic-Artikeln darauf hin, dass nicht nur Anthropomorphismus aufgrund der uns als Menschen eigenen Wahrnehmungs- und kognitiven Interpretationsprozesse unausweichlich ist, sondern unsere Sprache allein auf grammatischer Ebene bereits ,an anthropocentric view of the world around us" vorgebe. Sommer: Foremost in Creation, S. 53. Siehe auch Abschn. 4.5.
} 
Traditionen - hier der Konnex von ,Weichherzigkeit' gegenüber Tieren und Denkmodellen, die eine evolutionär bedingte Kontinuität zwischen Mensch und Tier als Grundlage haben und daher anthropomorphisierende Schlüsse ziehen, die als Fiktion ausgelegt werden; dort der Konnex von ,Unnachgiebigkeit" mit Realitätsbewusstsein und dem ideologischen Bemühen, keinerlei Kontinuität von Tier und Mensch in Denkmodellen zuzulassen - ist jedoch für die Gestaltung von wissenschaftlichen Texten ausschlaggebend. Dies zeigt sich an den unterschiedlichen ,Ecken' dieser Auseinandersetzung zuzurechnenden Texten von Wolfgang Köhler und Solly Zuckerman.

\subsubsection{Wolfgang Köhlers Intelligenzprüfungen (1917)}

Als Leiter der Anthropoidenstation auf Teneriffa führt Wolfgang Köhler von 1914 an Versuche mit Schimpansen durch. ${ }^{15}$ Im Jahr 1917 erscheint Intelligenzprüfungen an Anthropoiden. Bei den titelgebenden Intelligenzprüfungen handelt es sich um Experimente zum Problemlösungsverhalten mit sieben Versuchstieren, die nach folgendem Muster erfolgen:

Der Versuchsleiter stellt eine Situation her, in welcher der direkte Weg zum Ziel nicht gangbar ist, die aber einen indirekten Weg offenläßt. Das Tier kommt in diese Situation, die (der Möglichkeit nach) völlig überschaubar ist, und kann nun zeigen, bis zu welchem Verhaltenstypus seine Anlagen reichen, insbesondere ob es die Aufgabe auf dem möglichen Umweg löst. (3)

In diesen Experimenten wird ein bevorzugtes Futter (das ,Ziel ${ }^{`}$ ) mittels verschiedener Aufbauten außer Reichweite aber im Gesichtsfeld der Affen angebracht und es werden verschiedentlich Werkzeuge zur Verfügung gestellt, mit denen das Futter zu erreichen wäre. Das Verhalten der Tiere in dieser (,überschaubaren') Situation wird beobachtet und aus diesen Beobachtungen wird auf den kognitiven Prozess geschlossen, der zur Problemlösung führt.

„Zweierlei Interessen“ (1) macht Köhler für diese Forschungsarbeit geltend. Zum einen begreift er Menschenaffen als ,Wesen [...], welche dem Menschen in mancher Hinsicht näher stehen als sogar den übrigen Affenarten“ und ,der Beobachtung eine solche Fülle menschlicher Züge im sozusagen alltäglichen Verhalten“ zeigen, ,daß die Frage sich von selbst ergibt, ob diese Tiere auch in irgendeinem Grade verständig und einsichtig zu handeln vermögen, wenn die Umstände intelligentes Verhalten erfordern“(1). Zum anderen, dies nennt Köhler ein Ziel „theoretischer Art“, kann der „Anthropoide“ dadurch, dass er bei aller Menschenähnlichkeit doch ,weit hinter dem Menschen zurückbleibt“, unter einfachsten

\footnotetext{
${ }^{15}$ Genau genommen handelt es sich um Schimpansen und Bonobos. Eines der Tiere trägt den Namen „Tschego“, da man es „für ein Exemplar der Tschego-Abart“ (IM, S. 3) hält, wie sie Paul Du Chaillu in seinem Afrikareisebericht bereits als nshiego mbouve angibt (EA, S. v; siehe auch Abschn. 2.1.1). Bonobos sind jedoch erst ab 1929 als eigene Art (pan paniscus) klassifiziert und Köhler spricht abgesehen von dieser einen Erwähnung durchweg von Schimpansen.
} 
Verhältnissen die Natur von Intelligenzleistungen deutlich hervortreten lassen, während wenigstens der erwachsene Mensch, als Objekt der Selbstbeobachtung, einfache und deshalb an sich zur Untersuchung geeignete Leistungen kaum je neu vollzieht, und als Subjekt kompliziertere nur schwer hinreichend zu beobachten vermag. (1)

In den Intelligenzleistungen von Anthropoiden sollen Vorgänge plastisch anschaulich werden, ,die für uns zu geläufig geworden sind, als daß wir noch unmittelbar ihre ursprüngliche Form erkennen könnten, die aber wegen ihrer Einfachheit als der natürliche Ausgangspunkt theoretischen Verstehens erscheinen“ (1).

Erstens also soll die Problemeinsicht der Menschenaffen erforscht werden: Die Affen werden auf Intelligenzleistung hin geprüft. Zweitens aber sollen daraus die Voraussetzungen für die höher entwickelte Einsichtsfähigkeit beim Menschen hergeleitet werden, auf deren Erforschung der Mensch selbst - ähnlich wie Kafkas evoluierter Rotpeter auf sein Affentum ${ }^{16}$ - nicht mehr zurückgreifen kann. Julika Griem sieht daher Köhlers Forschung als wichtigen Grundstein für die moderne, überwiegend am Affen den Menschen erforschende Primatologie und verortet ihn im Rahmen eines Paradigmenwechsels des Bildes von insbesondere den großen Menschenaffen, der sich im frühen 20. Jahrhundert vor dem Hintergrund eines generellen ,symptomatischen Einstellungswechsel[s] im Verhältnis zu Wildtieren" 17 vollzieht. Nach dem wissenschaftlich und kulturell in der , anthropological anxiety" lange nachbebenden Schock der darwinistischen Theorie im 19. Jahrhundert wird nun vor allem das epistemologische Potenzial der Verwandtschaft von Affe und Mensch erkannt. Dies macht sich u. a. im Anstieg an Forschung mit Affen bemerkbar. Voraussetzung dafür ist, wie Köhlers obige Ausführungen in der Einleitung der Intelligenzprüfungen zeigen, die Konstruktion eines Modells von Menschenaffen - jene „Wesen [...], welche dem Menschen in mancher Hinsicht näher stehen als sogar den übrigen Affenarten“ (1) - als Vorläufer des Menschen. Nur so lässt sich mittels Interpretationsverfahren Aufschluss über die Mensch und Affe gemeinsamen kognitiven Prozesse gewinnen, welche zugleich als Teil der Genese menschlicher Intelligenz betrachtet werden können. ${ }^{18}$ Wegen des in einem solchen Modell witterbaren Anthropomorphismusverdachts assoziiert Julika Griem Köhlers Forschung mit der „Perspektive empathischer Einfühlung“19 und der „empathische[n] Betrachtungsweise“ ${ }^{20}$ Alexander Sokolowskys und Jakob von Uexkülls.

\footnotetext{
${ }^{16} \mathrm{Vgl}$. Kafka, Franz: Ein Bericht für eine Akademie und andere Texte zum Rotpeter-Thema. In: Ders.: Die Erzählungen und andere ausgewählte Prosa. Hg. von Roger Hermes. Frankfurt a. M. 1997, S. 322-337. Siehe auch Griem: Monkey Business, S. $144 \mathrm{ff}$.

${ }^{17}$ Griem: Monkey Business, S. 137.

${ }^{18}$ Zur Bedeutung der Modellbildung für den Tierversuch siehe Borgards, Roland: Das Tierexperiment in Literatur und Wissenschaft. Themen, Methoden, Theorien. In: Michael Gamper (Hg.): Experiment und Literaur. Themen, Methoden, Theorien. Göttingen 2010, S. 345-360.

${ }^{19}$ Griem: Monkey Business, S. 137.

${ }^{20}$ Griem: Monkey Business, S. 138.
} 
Statt der „romantisierende[n] Anthropomorphisierung“, 21 die Griem an Sokolowskys Beobachtungen über die Psyche von Menschenaffen ${ }^{22}$ diagnostiziert, findet sich bei Köhler jedoch eine das Stichwort aufgreifende Auseinandersetzung mit der Interpretation tierlichen Verhaltens und Erlebens. Wie die obigen Zitate zeigen, versteht Köhler unter Anthropomorphisierung ein „Hineinlegen in das Tier", von dem er seine eigenen Wendungen abgrenzt, denn diese bezieht er nach eigener Aussage stets auf das Verhalten, nicht das Bewusstsein des Tieres (vgl. 33, 73). Damit differenziert Köhler nicht nur zwischen Verwendungsweisen anthropomorphisierender Formulierungen. Er wendet sich auch - u. a. mit dem Hinweis auf unangebrachte Ängstlichkeit in Bezug auf Menschenähnlichkeit - gegen den Schaden, den eine Verweigerung jeder Art von ,Ein-Denken' in das Tier für die Forschung bringen kann, sofern sie ausgerechnet in einen paradoxerweise anthropomorphismuskritischen Anthropozentrismus führt. In den Ausführungen zur Werkzeugherstellung an späterer Stelle in den Intelligenzprüfungen wendet er sich gegen jene Paradoxie, die darin liege, jeden Hinweis auf ein Innenleben von Tieren zu schmähen, ihre kognitiven Leistungen und den experimentellen ,Erfolg' jedoch im Maßstab des Menschen zu berechnen. Die Interpretation der Leistungen der Schimpansen in den Versuchen dürfe nicht aufgrund unseres eigenen Werkzeuggebrauchs erfolgen: „Man muß sich hier, wie stets bei der Untersuchung des Schimpansen, davor hüten, den äußeren Eindruck von Menschenähnlichkeit (womöglich vom Werkzeug her induzierter) mit dem Niveau der Leistung, dem Grad der Einsicht zu verwechseln“ (118). Denn man muss, so Köhler,

\begin{abstract}
immer auf die Funktion ausgehen, in der das Tier den Gegenstand verwendet, muß herausfassen, was es davon wirklich übersieht: und wenn man erst weiß, welcherart die Funktionen sind, innerhalb deren der Schimpanse verstehen kann, was ein Gegenstand funktionell wert ist, so wird man lieber in diesem Gebiet einfachster, schlichter Zusammenhänge genauer untersuchen, was das Tier klar leistet und wie es dabei auf seine Lösungen kommt, als es mit komplexen Artefakten des Menschen zusammenzubringen. (119, Hervorheb. im Original)
\end{abstract}

Köhler scheint hier ein neueres Verständnis des Anthropomorphismus vorwegzunehmen: Als unumgängliche epistemologische und durch die menschliche Neigung zur Empathie bestimmte Voraussetzung des menschlichen Denkens lassen sich Anthropomorphismen nicht umgehen - sehr wohl aber kritisch reflektieren. Ähnlich wie es heute in Forderungen zu einem reflektierten, nicht-anthropozentrischen Anthropomorphismus ${ }^{23}$ diskutiert wird, nutzt Köhler 1917 , Theory of

\footnotetext{
${ }^{21}$ Griem: Monkey Business, S. 138.

${ }^{22}$ Sokolowsky, Alexander: Beobachtungen über die Psyche der Menschenaffen. Frankfurt a. M. 1908.

${ }^{23}$ Siehe bspw. Borgards, Roland: Observing Ethologists Observing Animals. Vortrag im Rahmen des Workshops Minds of Animals. Reflections on the Human-Non-Human Continuum. Universität Bern 2016; Borgards: Herzi-Lampi-Schatzis Tod, S. 78. Siehe auch Ingensieps Beschäftigung mit dieser Passage bei Köhler. Ingensiep: Der kultivierte Affe, S. 188 f.
} 
Mind“ als Erkenntnisinstrument für die Darstellung seiner Forschungsarbeit, statt ,romantisierenden Anthropomorphismen` zu erliegen. Als Teil der Gegenstände der neueren Empathieforschung lässt sich unter ,Theory of Mind' die Fähigkeit vestehen, „Bewusstseinsvorgänge bei sich selbst und bei anderen Lebewesen anzunehmen, um auf dieser Basis Vermutungen über das Befinden, das Vorhaben und die Motivation von anderen zu bilden“. ${ }^{24}$ Allein schon Köhlers Forschungsanliegen gibt einen solchen methodischen Ansatz vor. Muss er doch, um Einsicht in kognitive Prozesse zu erhalten, davon ausgehen, dass seine Forschungstiere nicht im Sinne des zeitgleich entstehenden Behaviorismus Reiz-ReaktionsMaschinen ähneln, sondern mit Karsten Stuebers Definition von ,basic empathy“ „minded creatures, [...] creatures that are fundamentally like us“, ${ }^{25}$ sind.

Dies zeigt sich auch daran, dass er sein Versuchspersonal individuell differenziert und die experimentellen Ergebnisse eher komparatistisch als universalisierend interpretiert. In der Einleitung zu den Intelligenzprüfungen stellt er seine jugendlichen Versuchstiere namentlich vor. Es handelt sich um sechs Weibchen (Tschego, Tercera, Rana, Nueva, Grande und Chica) und drei Männchen (Sultan, Konsul und Koko). Darunter hebt er einige aufgrund ihres Aussehens und ihrer Persönlichkeit hervor: Nueva „unterschied sich körperlich von diesen durch ihr merkwürdig breites, unschönes Gesicht und eine (offenbar pathologische) Dürftigkeit der Körperbehaarung über schlechter Haut““ (4). Ihre „Häßlichkeit“ sieht Köhler jedoch ,reichlich ausgeglichen durch ein Wesen [...] freundlicher Milde, naiven Zutrauens und stiller Klarheit" (4). Köhler zeigt sich beeindruckt von der ,feine[n] Art des Tieres, mit den einfachsten Mitteln stundenlang zufrieden zu spielen“ (4). Weitere Hinweise auf die „Gefühlspsychologie“ (123), wie es der Stumpf-Schüler Köhler später nennt, betreffen ihre Empörung, wenn man sie davon abhält, ihren Kot zu fressen, und ihren Zorn, wenn man ihr Futter vorenthält. Der 3-jährige Koko dagegen hat ,über dem stets prallen Bauch ein hübsches Gesicht mit ordentlichem Scheitel, mit spitzem Kinn und vordringenden Augen, das fortwährend unzufrieden zu fordern schien und dadurch dem kleinen Burschen etwas von selbstverständlicher Frechheit verlieh“ (5). Auch Koko wird über seine Affektpersönlichkeit charakterisiert, so ,verlief ein großer Teil seines Daseins in

\footnotetext{
${ }^{24}$ Curtis, Robin: Einführung in die Einfühlung. In: Robin Curtis/Gertrud Koch (Hg.): Einfühlung. Zur Geschichte und Gegenwart eines ästhetischen Konzepts. München 2009, S. 11-30, hier S. 12. Siehe auch Abschn. 3.1.

${ }^{25}$ Stueber, Karsten R.: Rediscovering Empathy. Agency, Folk Psychology, and the Human Sciences. Cambridge, Mass./London 2006, S. 4. Stueber argumentiert für ein Verständnis von Empathie als epistemisch zentrale Methode für unser Verständnis anderer Akteure: „Mechanisms of basic empathy have to be understood as mechanisms that underlie our theoretically unmediated quasi-perceptual ability to recognize other creatures directly as minded creatures, and to recognize them implicitly as creatures that are fundamentally like us" (S. 20). Obwohl Stuebers Verteidigung der Empathie als grundsätzliches epistemisches Instrument sich überwiegend mit dem empathischen Verständnis anderer Menschen beschäftigt, öffnen seine Formulierungen (,other agents', ,other creatures') die Diskussion hinsichtlich einer Ausweitung auf das Verständnis anderer Tiere.
} 
einer Art chronischer Empörung“; „er klagte nicht, er war entrüstet“ (5). Der in Köhlers Versuchen erfolgreichste Affe Sultan gilt dagegen als „Egoist [...] par excellence“"(5).

Diese Individualisierung der Versuchstiere ließe sich mit Griems an Sokolowsky gerichteten Vorwurf der ,romantisierenden Anthropomorphisierung ‘ lesen. Doch Köhler setzt sie keineswegs naiv ein, sondern nutzt diese Individualisierung, um eine weitere Prämisse seiner Forschungsarbeit zu verdeutlichen: Köhlers Affenmodell setzt zwar das Individuum insofern als Gattungsvertreter, als aus der durchschnittlichen Leistung der Versuchstiere Aufschluss über die Fähigkeiten der Spezies, dem ,gewöhnlichen Schimpansentypus“ (4), gewonnen werden soll. Köhler führt mit den verschiedenen Versuchstieren die gleichen Versuche aus und analysiert dabei den Werkzeuggebrauch und die Werkzeugherstellung beim Problemlösen, reflektiert die Umstände von ,Zufall und ,Nachahmung ' und den Umgang mit Formen und verändert den Versuchsaufbau, um Variationen zu provozieren. Detailliert werden der Versuchsaufbau und die Möglichkeiten der Problemlösung, das experimentrelevante Verhalten der Tiere und die Beobachtungen des Pflegepersonals wiedergegeben. Dabei legt Köhler aber Wert darauf, den Individualismus der Tiere in den Versuchen wiederzugeben. Trotz generalisierender Formulierungen wie „Wenn der Schimpanse“ $(88$, 96) werden die einzelnen Beobachtungen auch unter Namensnennung mit einzelnen Tieren und ihrem unterschiedlichen Verhalten und auch Wesenszug verbunden. Unterschiede in der Herangehensweise werden so auch individuell (,Die Kiste wird nicht steilgestellt; infolgedessen ist für den schlechten Springer Sultan der Stock durchaus notwendig“, 33) und mit ,,vollständig verschiedene[n] ,Persönlichkeiten““ (4) begründet. So kommt Köhler zum dem Schluss: ,[A]ls allgemeine Maxime kann man aufstellen, daß niemals Beobachtungen an nur einem Schimpansen als maßgebend für die Tierform überhaupt angesehen werden dürfen“"(5).

Zudem fällt auf, wie detailliert in den Intelligenzprüfungen die Versuche mit den einzelnen Tieren wiedergegeben werden:

Die sechs jungen Tiere des Stationsstammes werden in einem Raum mit glatten Wänden eingesperrt, dessen Decke (etwa $2 \mathrm{~m}$ hoch) sie nicht erreichen können; eine Holzkiste $(50 \times 40 \times 30 \mathrm{~cm})$, einerseits offen, steht etwa in der Mitte des Raumes, flachgestellt, die eine offene Seite vertikal gerichtet; das Ziel wird in einer Ecke (auf dem Boden gemessen $21 / 2 \mathrm{~m}$ von der Kiste entfernt) ans Dach genagelt. Alle Tiere bemühen sich vergeblich, das Ziel im Sprung vom Boden aus zu erreichen; Sultan gibt das jedoch bald auf, geht unruhig im Raum umher, bleibt plötzlich vor der Kiste stehen, ergreift sie, kantet sie hastig in gerader Linie auf das Ziel zu, steigt aber schon hinauf, als sie noch etwa $1 / 2 \mathrm{~m}$ (horizontal) entfernt ist, und reißt, sofort mit aller Kraft springend, das Ziel herunter. Seit Anheften des Zieles sind etwa 5 min vergangen; der Vorgang vom Stehenbleiben vor der Kiste bis zum ersten Biß in die Frucht hat nur wenige Sekunden gedauert, er ist, von jener Unstetigkeit (Stutzen) an, ein einziger glatter Verlauf. Vor jenem Augenblick hat sich keins der Tiere um die Kiste gekümmert, sie waren alle zu sehr mit dem Ziel beschäftigt; keines von ihnen hat auch den mindesten Anteil am Kistentransport - außer Sultan, der ihn eben in wenigen Augenblicken allein besorgt. Der Beobachter sah in diesem Versuch von außen durchs Gitter zu. (28 f.) 
Am Tage, nachdem Sultan die Kiste zum zweitenmal verwendet hat, wird das Ziel an dem weit höheren Dach eines anderen Raumes angebracht; zwei Kisten stehen etwa $5 \mathrm{~m}$ entfernt nahe beieinander auf dem Boden. Sultan, diesmal allein, kümmert sich zunächst nicht um die Kisten, sondern versucht mit einer kurzen, später einer längeren Stange das Ziel herunterzuschlagen; da die schweren Stöcke unsicher in seiner Hand schwanken, wird er bald ungeduldig und wütend, trampelt gegen die Wände und schleudert die Stöcke fort. Danach setzt er sich ermüdet auf einen Tisch, der in der Nähe der Kisten steht, und beginnt, als er sich erholt hat, ruhig um sich zu blicken, indem er langsam seinen Kopf kratzt; sein Blick fällt auf die Kisten und ruht einen Moment auf ihnen, schon klettert er auch vom Tisch herab, ergreift die nähere, zerrt sie unter das Ziel, besteigt sie aber erst, nachdem er seinen Stock aufgenommen hat und schlägt nun mühelos das Ziel herab. (33)

Die genauen Angaben zur Einrichtung des Versuchsraumes, der Ausstattung und Durchführung des Versuches, zu den Bewegungsfolgen, Zeitangaben und dem affektiven Zustand sowie die Erwähnung der Beteiligten und der Beobachterposition dienen einem bestimmten Verfahren der Darstellung: In der Schilderung gleichen die einzelnen Versuche in den Intelligenzprüfungen theatralen Situationen. Köhler gestaltet diesen Bühnen- und Aufführungscharakter des Experimentierens deutlich aus: ${ }^{26}$ Die schimpansischen Figuren treten teilweise geradezu auf- und ab; es ist vom „Zuschauer“ die Rede, der das Geschehen beobachtet. Nur vorübergehend auf dem Weg zu einem anderen Verhalten beobachtete Bewegungsfolgen werden als „Intermezzo“ (37) bezeichnet. Der Text ist mit Skizzen angereichert, die Aufbauten (Skizze 1, S.6), Boden- oder Raumpläne (Skizzen 2 u. 3., S. 9; Skizze 5 u. 6, S. 15 f.), Bewegungsmuster (Skizze 4, S. 11) u. ä. zeigen. Selbst die eingefügten Fotographien gleichen Szenenbildern. Zugleich wurden die Experimente gefilmt, um nicht allein auf die Verschriftlichung angewiesen zu sein, sondern ein nicht diesem Zwischenraum zwischen der experimentellen Anordnung und dem „Raum der gedruckten Mitteilung “27 zugehöriges Abbildeverfahren nutzen zu können. Diese Durchdringung der Experimentalität mit der Theatralität allein schon im Versuchsaufbau hat Esther Köhring bereits untersucht. „Um Einsicht sichtbar zu machen“, so Köhring, gestalte Köhler ,naturalistische Szenen“ ${ }^{28}$ in denen die Affen nicht einen

\footnotetext{
${ }^{26}$ Damit kommt Köhler der „Hypothese einer fundamentalen Theatralität des Experiments“ entgegen, die davon ausgeht, „dass Wissensgenerierung an spezifische Verfahren der Konstruktion und Inszenierung geknüpft ist““. Müller-Wille, Klaus: Inszeniertes Wissen. Theater und Experiment. In: Michael Gamper (Hg.): Experiment und Literatur. Themen, Methoden, Theorien. Göttingen 2010, S. 40-68, hier S. 43.

${ }^{27}$ Rheinberger, Hans-Jörg: Wissensräume und experimentelle Praxis. In: Helmar Schramm u. a. (Hg.): Bühnen des Wissens. Interferenzen zwischen Wissenschaft und Kunst. Berlin 2003, S. 339-382, hier S. 372. Zum Filmmaterial der Versuche siehe Köhler, Wolfgang: Wolfgang Köhler: Intelligenzprüfungen an Menschenaffen. Film- und Fotoarchiv des Adolf-WürthZentrums für Geschichte der Psychologie, http://www.awz.uni-wuerzburg.de/archiv/film-fotoarchiv/wolfgang-koehler/ (16.04.2018).

${ }^{28}$ Köhring, Esther: Einsicht einsichtig werden lassen. Experimentelles Theater und Tierexperiment in Samuel Becketts Acte sans Paroles I und Wolfgang Köhlers Intelligenzprüfungen an Menschenaffen. In: Roland Borgards/Nicolas Pethes (Hg.): Tier - Experiment - Literatur. 1880-2010. Würzburg 2013, S. 109-126, hier S. 115.
} 
anonymen Knopf drücken, sondern in Situationen (bedingt) freien Werkzeuggebrauch üben können. Köhring sieht im Auf- und Ausbau dieser Situationen, in denen ,entgegen Köhlers Beschreibungen die eigentliche Gelingensbedingung nicht der Überblick (für das Tier) ist, sondern die Überblickbarkeit (für den Beobachter)“, ${ }^{29}$ die erst den Eindruck entstehen lässt, Einsicht in die Einsicht sei möglich, einen „stets drohenden Anthropomorphismus in das gesamte System distribuiert und systematisch naturalisiert“, sodass „der Beobachter entlastet und objektiviert" ${ }^{* 30}$ sei. Für Köhlers Anliegen sei dieser theatrale Aufbau essenziell, denn „Köhlers Einsichtsmomente (sind) nicht abzähl- und messbar, sondern bedürfen der Rahmung, Narrativierung und Inszenierung, um sicht- und einsichtbar zu werden“. ${ }^{31}$ Für Köhring folgen die Versuchsbeschreibungen daher einer ,iterativen Dramaturgie, die selbst Evidenzwert beansprucht und zum poetologischen Prinzip wird“. ${ }^{32}$ Köhring spricht hier von einer Ästhetik, die sich „durch eine betonte Textualität bei geleugneter Literarizität, durch eine an-, aber nicht ausgespielte Performativität und durch eine Äußerlichkeit der Beschreibung statt einer Einfühlung in das Versuchstier“33 auszeichne.

Köhrings um die (Dis-)Kontinuität von Theatralität und Experimentalität zentrierter Analyse des Köhler-Textes ist in vielen Punkten zu folgen. Besonders ihr Verweis auf die ,Äußerlichkeit der Beschreibung' ist wichtig. Doch scheint mir die kritische Perspektive auf Köhlers Intelligenzprüfungen hier das ,statt“ in Konjunktion mit der Einfühlung in das Versuchstier zu stark zu betonen. ${ }^{34}$ Diese ,äußerliche' Darstellungsweise, so lässt sich behaupten, steht im Kontext des, wie Köhring selbst schreibt, stets drohenden Anthropomorphismus-Vorwurfs. Die Äußerlichkeit der Beschreibung lässt sich nämlich auch als eine Strategie des wissenschaftlichen Erzählens betrachten, die die Narration einer Einfühlung ins Versuchstier umgeht, ohne die Einfühlung selbst auszuschließen. Sicherlich wird dabei schreibend die ,Einsicht in die Einsicht' konstruiert, wie Köhring aufzeigt. Es wird aber vor allem die empathisch generierte Interpretation des experimentell hergestellten Problemlösungsverhaltens aus den äußeren Umständen, somit ,empirisch', herausgearbeitet. Im Gegensatz zur unter dem Anthropomorphismus-Verdacht kaum salonfähigen

\footnotetext{
${ }^{29}$ Köhring: Einsicht einsichtig werden lassen, S. 116.

${ }^{30}$ Köhring: Einsicht einsichtig werden lassen, S. 116.

${ }^{31}$ Köhring: Einsicht einsichtig werden lassen, S. 116.

${ }^{32}$ Köhring: Einsicht einsichtig werden lassen, S. 116.

${ }^{33}$ Köhring: Einsicht einsichtig werden lassen, S. 120.

${ }^{34} \mathrm{Im}$ Folgenden verwende ich aus historischen Gründen weitgehend den von Köhring angeführten Begriff der Einfühlung, obwohl im Kontext primatologischer Schriften diesem weiterhin überwiegend der Ästhetik verhafteten Begriff jener der Empathie im Verständnis neuerer Forschung vorzuziehen ist. Vgl. zur Begriffsgeschichte Curtis: Einführung; Fontius, Martin: Einfühlung/Empathie/ Identifikation. In: Karlheinz Barck (Hg.): Ästhetische Grundbegriffe: Historisches Wörterbuch in sieben Bänden. Bd. 2. Stuttgart/Weimar 2001, S. 119-142; Coplan, Amy/Goldie, Peter: Introduction. In: Dies. (Hg.): Empathy. Philosophical and Psychological Perspectives. Oxford/New York 2011, S. ix-xlvii.
} 
Einfühlung wird damit eine in der zeitgenössisch im Umbruch befindlichen wissenschaftlichen Kultur zulässige Erkenntnisproduktion vorgegeben. ${ }^{35}$ Köhler verwendet hier seinerseits einen Umweg, um an sein Ziel zu gelangen: Durch die Konzentration auf die Äußerlichkeit der Beschreibung kann den Tieren in kleinen Dosen ein Innenleben zugeschrieben werden, das den Doppelsinn hat, sie als handelnde Figuren in den Experiment-Szenen auszugestalten und Ansätze für die Erläuterung ihrer Handlungsweisen zu bieten, die aus der Beobachtung nur in Kombination mit ,Theory of Mind“ ergehen können. So bleibt Sultan „,eine Weile mißmutig liegen“, nachdem er „mehrere Male mit ihr [einer Leiter] zu Boden gefallen ist“" (114, Hervorheb. MS). In Zusammenhang mit Konsuls „Gewohnheit, hinter einem anderen Tier herzugehen, indem er beide Hände auf des Vordermannes Schultern legt und nun die Beine im Gleichschritt mit dem anderen Tier vorwärtsetzt", heißt es über Grande, auf deren Rücken er aus dieser Position heraus zu steigen versucht, dass sie „offensichtlich nicht ahnt, was er vorhat" (36, Hervorheb. MS) und daher am Ziel vorbeigeht. Als Konsul versucht, Tercera und Rana als Kiste zu benutzen, nehmen ,beide mit allen Zeichen der Bestürzung vor ihm Reißaus [...]; sie verstehen sichtlich nicht, weshalb er fortwährend mit ausgestreckter Hand hinter ihnen herläuft und fürchten Böses“" (36, Hervorheb. MS).

Die Auswertung dieser Beobachtungen (bzw. die Interpretation der Szenen) ist teilweise auf spätere Kapitel von Köhlers Studie verschoben, die generalisierend aus den Experimenten schöpfen. Im Rahmen theoretischer Überlegungen kann Köhler dabei stärker mit Zuschreibungen arbeiten, die es ermöglichen, die Tiere auch als emotionale Subjekte zu verhandeln. Beispielsweise wird Grande in der Erregung über einen neuen Versuchsleiter (Köhler selbst in der Anfangszeit) im Kapitel zum Werkzeuggebrauch mit einem Stock so zum „säbelschwingenden Raufbold“ (60) und führt eine Art „Grausamkeitsspiel“ (60) auf, das folgenlos bleibt. Gerade die Verwendung von Werkzeugen „,[i]n großer Freude“ (61), ,,auch in großem Zorn“ (62), als ,heftig[e] Gefühlsäußerung“ (62) und nicht als „Waffengebrauch“ (62) betont Köhler. Hier schleicht sich die Tierpsychologie wieder in die vermeintliche Ästhetik der Äußerlichkeit hinein. ${ }^{36}$ Dies geschieht über eine gefühlspsychologische Deutung. So heißt es:

\footnotetext{
${ }^{35}$ Dass eine derartige ,Taktik‘ in der Verschriftlichung vorliegt, sieht auch Köhring. Im Zusammenhang mit Köhlers Produktion von Begriffen aus Momenten (,Fußanheben', ,Kiste-an-die-Wanddrücken'), geht sie auch darauf ein, dass der Experimentator hier „,von der Verantwortung der anthropozentrischen Sprache entlastet [sei] und [...] den Moment der Einsicht als nicht-sprachlich, ereignishaft behaupten“ könne. Köhring: Einsicht einsichtig werden lassen, S. 123. Zur Entwicklungsgeschichte des Behaviorismus, der, verwurzelt in Pavlovs Konditionierungsversuchen, mit John B. Watsons Manifest Psychology As the Behaviorist Views It 1913 erstmals namentlich in Erscheinung tritt, vgl. Weidman, Nadine: Behaviorism. In: Maryanne Cline Horowitz (Hg.): New Dictionary of the History of Ideas. Detroit u. a. 2005, S. 205-209; Watson, John B.: Psychology as the Behaviorist Views It. In: Psychological Review 20 (1913), S. 159-177.

${ }^{36}$ Zum Einfluss von Wolfgang Köhlers Gestaltpsychologie auf die Tierpsychologie Konrad Lorenz' und Nikolaas Tinbergens vgl. Kressley, Regina A.: Gestalt Psychology: Its Paradigm-Shaping Influence on Animal Psychology. In: Gestalt Theory 28/3 (2006), S. 259-268.
} 
Zornige Erregung ist jedoch gar nicht der günstigste Fall für die Beobachtung der sehr allgemeinen Erscheinung, um die es sich hier handelt; an sich vielleicht schwächere Affekte, die aber länger andauern als der schnell vergängliche Zorn, haben mehr Zeit, alle in ihnen liegenden Möglichkeiten zu entwickeln. (62)

Dann schildert Köhler, wie sich ein Schimpanse in der Isolation verhält, wenn die „Kameraden“ „nicht gleich an sein Gitter, an sein Fenster“ kommen, „um ihn zu umarmen, wie er jammert und heult" (64):

[D]a streckt er die Arme mit bittenden Bewegungen hinaus in ihre Richtung, und wie sie noch nicht kommen, stopft er seine Decke, Stroh, oder was sonst in seinem Raume liegt, zwischen den Stäben hindurch und schwenkt das alles in der Luft, aber immer nach den andern zu; schließlich, in dem größten Kummer, wirft er einen Teil der ihn umgebenden Mobilien nach dem andern in Richtung seiner Sehnsucht hinaus. (64)

Dies ist nicht mehr nur ein verleitender anthropozentrischer Sprachgebrauch, wie er bei den Beschreibungen von Bewegungen zum Tragen kam und wie ihn Köhler im Zusammenhang des Anthropomorphismus-Verdachts zu rechtfertigen wusste. Köhler diagnostiziert in einem zufälligen Affekt-Experiment - die Isolation ist nicht Selbstzweck, ihr Grund sind später anstehende Versuche - Emotionen: Kummer und Sehnsucht. Die ganze Szene wird sogar in Linie mit Köhlers Theatralisierung der Beschreibung zur schimpansischen Pathosformel, jenem potenziell theatralen Ausdruck starker affektiver Erregung als Körperbild des flehentlich ausgestreckten Armes. ${ }^{37}$ Nach zwei weiteren Beispielen, bei denen u. a. geheult, (bittend) Arme ausgestreckt, (aufgeregt) gehopst, (nervös) am Kopf gekratzt, (wütend) an die Wand geschlagen, und (frustriert) mit Stöckchen und Steinchen nach einer erwünschten Kontaktperson (und ihrer Portion Bananen) geworfen wird, heißt es sogar deutlich: „In allen drei Fällen ist es keinesfalls notwendig, auch gar nicht die Regel, daß der Zustand des Tieres in ohnmächtige Wut übergeht; sie sind dabei nicht zornig, sondern sehnen sich und wünschen" (64, Hervorheb. MS). Damit dieser der Einfühlung und des Anthropomorphismus verdächtige Satz neutralisiert wird, verlegt sich Köhler im Folgenden auf die Deutung von motivationalen, affektgesteuerten Bewegungsrichtungen:

Danach veranlaßt ein Wunsch, der räumliche Richtung hat, aber längere Zeit nicht erfüllt werden kann, schließlich Aktionen in jener Richtung, ohne viel Rücksicht auf praktischen Wert. [...] In starkem Affekt ohne Lösung muß das Tier etwas in die Raumrichtung tun, in der sein Wunschobjekt sich befindet; es muß sich schließlich irgendwie mit diesem in Verbindung setzen, wenn auch nicht praktisch-erfolgsmäßig, muß irgend etwas zwischen sich und ihm vollziehen, und wäre es so wertlos wie das Hinschleudern von beweglichen Gegenständen seiner unmittelbaren Umgebung. (64 f.)

\footnotetext{
${ }^{37}$ In dieser Szene ließe sich der Werkzeuggebrauch - das Schwenken von Decke, Stroh u. ä. durch die Stäbe hindurch in der Luft, das Werfen des Mobiliars nach den anderen Schimpansen - als Äquivalent der dem Körperbild korrespondierende Sprachgebärden lesen. Vgl. Port: Pathosformeln, S. 27. und Abschn. 2.2.
} 
Zusammengefasst lässt sich Wolfgang Köhlers epistemologische Ästhetik der Äußerlichkeit, wie Köhring sie identifiziert, als Strategie lesen, dem in der zeitgenössischen Wissenschaftskultur zunehmend schwerwiegenden, mit Sentimentalität und Unwissenschaftlichkeit konnotierten Anthropomorphismus-Verdacht etwas entgegenzusetzen. Durch die Notwendigkeit der in der theatralen Darstellungsweise bedingten Interpretation scheint die Lizenz verliehen, durchaus mittels Verfahren von ,Theory of Mind“ im Rahmen der Einfühlung Interpretationen zu liefern, die seinem Forschungsanliegen - der Einsicht in die Funktionsweisen des anthropoiden Problem-Bewusstseins - entsprechen.

\subsubsection{Solly Zuckermans The Social Life of Monkeys and Apes (1932)}

Solly Zuckerman veröffentlicht 1932 The Social Life of Monkeys and Apes, eine umfangreiche Ausarbeitung eines 1929 erschienenen Aufsatzes, die sich aus seinen in Südafrika angestellten Freilandbeobachtungen und der Zeit im Londoner Zoo speist, aber auch die Studien anderer Forscher heranzieht. Anders als Köhler, dessen Text eine Gruppe von durchgeführten Versuchen zusammenfasst, geht es Zuckerman um eine Überblicksdarstellung der Sozialstruktur von Affen und Menschenaffen, in seinem Vokabular: „sub-human primates“ (1). Zuckerman ist es ein Anliegen, sich von einem Forschungstypus, den er als ,,anecdotal method in animal psychology" (11) bezeichnet, abzugrenzen. Denn diese assoziiert er, wie oben schon angeführt, durch sein Vokabular (,web of romance“, „realms of imagination“, „the fanciful“, „stories“) mit dem Bereich des Fiktiven, und deren Anspruch auf Wissenschaftlichkeit bedenkt er gar, wie bereits zitiert (vgl. Abschn. 2.2.2), mit „ridicule“ (11). Die „experimental method“ dagegen ,promised results which, even if not interpreted alike by any two students, were yet capable of corroboration, since the conditions in which experiments are made can be controlled and adequately described" (11). Im ersten Kapitel wird der Eindruck der Erklärung aus dem Vorwort vertieft: die Ablehnung einer ,naiven', anekdotisch gespeisten, anthropomorphisierend denkenden Tierpsychologie und die Befürwortung einer der Tiernutzung verschriebenen experimentellen, physiologisch arbeitenden und positivistisch ausgerichteten Zoologie.

Diese Abgrenzung ist umso interessanter, als Zuckerman in seiner Darstellung streng genommen nicht von einzelnen Experimenten berichtet. Stattdessen basieren seine Überlegungen auf Referaten zum bestehenden Wissensstand über Affen und Studien zur geschlechtsspezifischen Anatomie. Ihr Kernstïck sind jedoch Beobachtungen: jene im Feld in Südafrika angestellten und jene aus dem Londoner Zoo. Wie Zuckerman berichtet, wurden im Frühling 1925 im Londoner Zoo ca. 100 Hamadryas-Paviane, davon 94 männliche und sechs weibliche Tiere, in ,a large oval rockwork enclosure one hundred feet long and sixty feet wide“ (218) namens ,Monkey Hill` ausgesetzt. Die Anzahl der Tiere dezimiert sich rasch: Nicht nur fordern Krankheiten viele Todesopfer; aufgrund des Missverhältnisses der Geschlechter, der großen Anzahl an Individuen und dem Mangel an 
territorialen Ausweichmöglichkeiten kommt es vor allem zu schweren Kämpfen und sehr vielen Todesfällen. ,Monkey Hill‘ lässt sich so als großes Sozialexperiment mit Pavianen verstehen. Zuckerman verweigert sich jedoch der Einsicht, dass das beobachtete Verhalten der Hamadryas-Paviane im Londoner Zoo drastisch von den Bedingungen, unter denen sie hier gehalten werden, und der Manipulation der Gruppen-Größe und -Zusammensetzung abhängt und daher kaum repräsentativ für die Art sein kann. Derartige Einwände tut Zuckerman als „,common belief“ ab und behauptet schlicht, ,that the new environment grossly distorts the expression of these [social] relationships has no foundation in fact" (216). Zwar muss er einräumen, dass es möglich sei, ,that captive conditions modify fighting behaviour“ und ,that fights may often be carried much farther in captivity than they would be in nature“, konstatiert aber: „This, however, adds to their interest. From the point of view of the observer, confinement concentrates a normal response both temporally and spatially“ (217). Zuckerman konzipiert hier die Affenkolonie im Zoo als insulare Möglichkeit eines kondensierten, Normalität repräsentierenden Verhaltensextrakts. ${ }^{38}$ Unterlegt werden diese Beobachtungen mit anatomischen Analysen, d. h. am toten Affenkörper gewonnenes physiologisches Wissen dient der Erläuterung und Begründung des beobachteten Verhaltens. Zuckermans Vertrauen in den unmittelbaren Zusammenhang von Anatomie und Verhalten, das hat die primatologische Auseinandersetzung mit der eigenen Fachgeschichte bereits zutage gefördert, ist eines, welches das Verhalten als sekundär betrachtet. ${ }^{39}$

\footnotetext{
${ }^{38}$ Zuckerman führt seinen programmatischen Ansatz diesbezüglich folgendermaßen aus: „A full display of the naturally orientated sexual responses of any sub-human primate species could only be observed in a homogenous and complete group of animals - a group consisting of individuals of all ages and both sexes, preferably from the same locality. The true significance of many forms of sexual behaviour, which appear meaningless when manifested in a cage, can be readily recognized in a social environment of this kind. Opportunities for studying such social groups have only recently arisen" (SL, S. 215 f.). Die Paviankolonie wird ebenso wie jene im Tierpark München oder die der Kolonialausstellung 1931 in Paris als Errungenschaft für die Forschung betrachtet. Für Zoo- und Biologiehistoriker/innen ist der Londoner Monkey Hill heute allerdings gerade als gescheitertes Tierhaltungsexperiment von großem Interesse. Siehe auch Burt, Jonathan: Solly Zuckerman: the making of a primatological career in Britain, 1925-1945. In: Studies in History and Philosophy of Biological and Biomedical Science 37 (2006), S. 295-310, hier S. 300 sowie Burt, Jonathan: Violent Health and the Moving Image: The London Zoo and Monkey Hill. In: Mary J. Henninger-Voss (Hg.): Animals in Human Histories: The Mirror of Nature and Culture. Rochester/Woodbridge 2002, S. 258-292.

${ }^{39}$ Dies äußert sich auch in einer auffallend vehementen Kritik Zuckermans an ethologischen Freilandstudien. Bereits in The Social Life mokiert sich Zuckerman über eine frühe Langzeitstudie von Eugène Nielsen Marais (SL, S. 205). Marais' posthume Publikation The Soul of the Ape (1969) wird von Zuckerman aufs Schärfste angegriffen, weil ihrem Autor in einem Vorwort Robert Ardreys eine vorausschauende Vorbereitung der primatologischen Feldforschung bescheinigt wird. Vgl. Morris, A. G.: Zuckerman versus Marais: a primatological collision. In: South African Journal of Science 105 (2009), S. 238-240; Marais, Eugène: The Soul of the Ape. New York 1969. Zuckermans Arroganz gegenüber der ethologischen Feldforschung zieht sich bis weit in die Zeit moderner Primatologie hinein, vgl. Rowell, Thelma: A Few Peculiar Primates. In: Shirley C. Strum/Linda Marie Fedigan (Hg.): Primate Encounters. Models of Science, Gender, and Society. Chicago/London 2000, S. 57-70, hier S. 59 f.
} 
Um den Zusammenhang von Anatomie und Verhalten - und damit seine Autorität als zoologischer Anatom auch für die Zwischenkriegs-Biologie - herzustellen, ist Zuckerman allerdings dazu genötigt, anekdotisches Material, oder in anderen Worten schlicht die Beobachtungen anderer, in seine Überlegungen aufzunehmen. ${ }^{40} \mathrm{Zu}$ diesem Zweck unterscheidet er zwischen der ,form of travellers' tales [...]; anecdotes in which factual and interpretative elements are inextricably mingled“(11), und dem Wissen von „farmers and trappers“, die ,know something about the life histories of those mammals that are of economic importance, either because of the damage they do to land and crops, or because of the worth of their fur" (11). Diesen die Tierbeobachtungen scheinbar legitimierenden ökonomischen Aspekt rückt Zuckerman dezidiert in den Vordergrund. Die Notwendigkeit ökologischer Studien ist bei ihm auch damit begründet, dass sie ,economic importance“ (12) für die Landwirtschaft haben. Besonders wichtig erscheint ihm dies in Bezug auf seine zentrale Spezies, den Pavian, den er bereits in diesem ersten Kapitel kategorial eine Schädlingsart nennt: „In South Africa baboons were at one time officially declared vermin because of the damage they do to farm lands. The Government subsidized the cost of their destruction" (12). Zuckermans Fokus auf die Reproduktion als zentrales Steuerungselement primatischen Sozialverhaltens mag auch im Kontext seiner eindrücklichen Ausbildungserfahrungen aus dem gynäkologischen Bereich entstanden sein, wie sein Biograph dies andeutet; ${ }^{41}$ er steht jedoch deutlich vor allem im Zusammenhang dieser Modellierung des Affen als Pavian und somit als Schädlingsart, bei der das Wissen vom reproduktiven Verhalten als Mittel der Kontrolle dienen kann. ${ }^{42}$

Das an der sexuellen Reproduktion ausgerichtete Schema des ,sub-humanen“ primatischen Sozialverhaltens ist im zweiten Kapitel (,Mammalian Sociology“, 17 ff.) bereits vollständig angegeben und ausgeführt:

\footnotetext{
The main factor that determines social grouping in sub-human primates is sexual attraction. Females attract males and males attract females. The limit to the number of females held by any single male is determined by his degree of dominance, which will again depend not only upon his own potency, but also upon his relationships with his fellow males. The occurrence of sexual individuals within a particular area will act as a stimulus to the expansion of the group, and the limits of expansion will be set both by the relationships within the group and by the environmental conditions within which the group lives. (31)
}

\footnotetext{
${ }^{40} \mathrm{Vgl} . S L$, S. $206 \mathrm{f}$.

${ }^{41}$ Vgl. Donovan: Zuckerman, S. 9.

${ }^{42}$ Zuckermans Freiland-Beobachtungen in Südafrika erfolgen im Kontext von ,hunting-parties", die ausziehen, den Schädling Pavian in möglichst großer Zahl zu töten. Dabei zeigt sich jedoch auch das Bedauern des Forschers über die Vernichtung seines Gegenstandes: Zuckerman bezeichnet diese durch die Regierung geförderte Maßnahme als „slaughter“ und scheint als Zoologe eine gewisse Freude daran zu haben, wenn ihm das Scheitern der Jagd mehr Zeit für die Beobachtung lässt (SL, S. 196 ff.).
} 
Gerade vor dem Hintergrund seiner Ablehnung von Anthropomorphismen ist es auffallend, dass diese soziale Form von Zuckerman bereitwillig mit einem deutlich anthropomorphisierenden Begriff belegt bezeichnet wird: „The social unit in these [baboon] colonies is the harem" (144, Hervorheb. MS). Präsidiert werden sie von einem ,overlord“ (144), dessen Ziel es ist, „to secure for himself as many females as possible“ (190). Ein typischer Satz bei Zuckerman lautet: „The oestrus female usually spends the day sitting and moving in close contact with her overlord, while the quiescent females are always more active and may wander some distance away from the overlord“ (144). Das Paradigma ,Harem“ mit seinen Konnotationen von, wie Fedigan es fasst, „, enslaved females' and male autocracy", ${ }^{43}$ wirkt so stark auf Zuckerman ein, dass es die Interpretation physiologischer Fakten prägt: „Evidence of severe bodily mutilation suggests strongly that the social life of the orang has its basis in the harem, which is maintained by the dominance of the overlord“ (179). Als er in Südafrika Chacma-Paviane beobachtet, die anders als die Londoner Hamadryas-Paviane nicht in polygynen Gruppen leben, bemängelt er sogar, dass „,no clear signs of leadership“ (201) zu sehen seien. Die Übertragung des (orientalistisch unterfütterten) sozialen Konzeptes ,Harem“ auf Tiere ist zeitgenössisch zoologisch so verankert, dass sie von Zuckerman nicht als anthropomorphisierendes Verfahren identifiziert wird. ${ }^{44}$ Es ist dennoch auffallend, wie oft Zuckerman trotz seiner Ablehnung anthropomorphisierender und anthropozentrischer Interpretationen in seinen Beschreibungen Vergleichbares implementiert. So wenn er die Konzepte Promiskuität, Untreue, Verführung und sogar Prostitution auf die Tiere anwendet:

\begin{abstract}
Baboons are not promiscuous. [...] None of these females has been seen to be ,unfaithful' during a phase of sexual swelling. [...] The quiescent phase of the sexual skin [...] presents more opportunity for infidelity. (228, Hervorheb. MS)
\end{abstract}

The first seducer in the contest that followed was a rather powerful unmated male. The fight was peculiar in so far as the overlord ignored his female after her initial seduction and took no further part in the fight. (257, Hervorheb. MS)

In an earlier chapter it was stated that the liberation of sexual responses from the function of reproduction may be regarded anthropomorphically as sexual prostitution [...]. Prostitution is not the interpretation of the process underlying the relevant responses - it is the interpretation of the actual results of the behaviour. Thus if a particular response of a sexual nature is always followed by the acquisition of some social or material advantage, it is legitimate, for purposes of description, to refer to the response as a form of sexual prostitution. (232 f., Hervorheb. MS)

\footnotetext{
${ }^{43}$ Fedigan: Primate Paradigms, S. 238.

${ }^{44}$ Zur Popularität des Harems als orientalistischem Topos vgl. Lewis, Reina: Rethinking Orientalism: Women, Travel and the Ottoman Harem. London/New York 2004, S. $12 \mathrm{ff}$. „[B]ecause of its anthropomorphic (and androcentric!) connotations“ ist der Begriff Harem für die von Zuckerman damit bezeichnete Sozialstruktur der heutigen deskriptiven Terminologie von ,polygynous group“ oder „uni-male, multi-female reproductive unit“ gewichen. Fedigan: Primate Paradigms, S. 41.
} 
Werden Untreue und Verführung schlichtweg übertragen, wird der Begriffstransfer der Prostitution argumentativ unterlegt: Sie wird dabei von der Motivation (,,processes underlying the relevant responses“) zur funktionalistischen Interpretation der Ergebnisse eines Verhaltens (,the actual results of the behaviour") umgedeutet und als legitime Beschreibungskategorie konstruiert. Zuckermans Unbehagen daran, im Rahmen seiner Anthropomorphismus- und Anthropozentrismuskritik bestimmte Konzepte nicht verwenden zu dürfen - aber sie nutzen zu wollen - wird durch rhetorische Volten und eine zunehmend anwachsende Syntax angedeutet.

In diesem Zusammenhang bietet sich eine andere Interpretation von Zuckermans Induktion aus dem Primatenkörper an. Zum einen ist Zuckermans anatomische und physiologische Expertise der Grund für den großen Raum, der den Themen Östrus, Menstruation etc. im Gesamtumfang des Social Life gewährt wird. Der anatomische Fokus steuert Zuckermans Wahrnehmung und Darstellung der Affen, ihres Verhaltens und der Forschungszusammenhänge, aber auch die Möglichkeiten des Textes. Zum anderen aber ist dieser Fokus auf das Anatomische und Physiologische, auf die Fakten des Körpers als ,Empirie“, aber auch eine Schutzfunktion, die ihn im Kontext des sich von strukturellem Funktionalismus zum Behaviorismus entwickelnden theoretischen Leitgedankens sicher einbettet und zugleich ein Ordnungsgeflecht anbietet, um die nicht unproblematische Forschungsgrundlage $\mathrm{zu}$ strukturieren und $\mathrm{zu}$ vereinheitlichen. Dies zeigt sich im Kapitel „The Hamadryas Baboon Colony“ (215 ff.), in dem mit der Londoner Monkey-Hill-Kolonie das Kernstück von Zuckermans Forschungsarbeit betrachtet wird. In diesen Jahren in London studiert Zuckerman den Niedergang der Paviankolonie durch massive Kämpfe und schließt aus der Autopsie verstorbener Tiere auf das dem Tod vorangegangene bzw. diesen provozierende Sozialverhalten. Bevor sich Zuckerman also der detaillierten Darstellung von Sexualverhalten unter den Pavianen widmen kann (225 ff.), muss er durch eine erläuternde Schilderung der Zustände am Monkey Hill ,hindurch“. Dass Solly Zuckerman von dieser fatalen Entwicklung unter seiner Forschungspopulation nicht unbeeindruckt bleibt, zeigt sich an seinen Formulierungen: „Early in 1927 the stock was still further depleted“, ,remaining population was only fitfty-six“; ,even more reduced“; ,the colony had again been thinned“ (219), teilweise bedauernd: „The males fight for the females, who are usually fatally injured in the mêlée which rages around them" (219). Doch die Konzentration auf physiologische Beobachtungen post mortem und die daraus verfügbaren Schlüsse auf vorangegangenes Verhalten erbringen einen affektbereinigten Darstellungsmodus. So heißt es beispielsweise:

Between June and December of 1925, there were twenty-seven deaths, twenty-six from recognizable pathological lesions on internal organs, and one from injuries received in fighting. Many of the animals whose deaths were attributed to organic disease also showed wounds indicating their recent participation in fights. For example, one animal, both of whose lungs were in pneumonic condition, had a suppurating wound on the left forearm, which may have been inflicted before or - since sick baboons are often attacked by their fellows - after the onset of its fatal illness [...]. (218 f.) 
The greatest number of deaths appeared in 1927, when the main consignement of animals were introduced. Twenty-six animals belonging to the 1925 batch died of disease within three and a half month of their arrival, and fifteen of the thirty females imported in 1927 were killed within a month. Judging from the total records of females that died from injuries, fighting seems to have occurred throughout the year. (220 f.)

Die Konzentration auf die Ergebnisse anatomischer Untersuchungen und die statistische Auflistung von Todesfällen und Verletzungen lässt den anonymen Ton einer wissenschaftlichen Neutralität entstehen, die die chaotische Unübersichtlichkeit der Vorfälle zu bändigen und zu interpretieren weiß. Zugleich verleiht ein solcherart ,szientistischer' Diskurs Zuckerman einige Lizenzen: So kann er sich erstens vor dessen Hintergrund hier und da einen reflektierenden Kommentar erlauben, um $\mathrm{zu}$ verdeutlichen, was die Gewaltakte bedeuten und welche Konsequenzen daraus zu ziehen sind: „The fight in which the last of these females lost her life was so protracted and repellent - from the anthropocentric point of view - that the decision was made to remove the five surviving females from the Hill" (219, Hervorheb. MS). Zweitens finden sich drastische Beschreibungen der Paviangewalt, die als Illustration zwischen den ,Daten' eingesetzt werden. So wenn Zuckerman erläutert, weshalb kaum eines der in der Kolonie geborenen Pavianbabys überlebt habe:

\begin{abstract}
Observations made on the Hill show that the young animals often die of strangulation. A young baboon clings to the hair on the belly of its mother, who supports it with her arms. When a fight breaks out on the hill, excitement may spread to all the animals, most of whom will then make for the centre of the fight. In the mêlée it is possible that a nursing baboon tightens her hold on her clinging infant and thus strangles it. There is a record of a nursing animal that dropped her baby in the excitement of a fight. The young animal was immediately seized around the neck by a male, who made off with it. (222)
\end{abstract}

Als es darum geht, dass manchmal schlicht mütterliche Unerfahrenheit für den Tod der Babys verantwortlich sei, heißt es:

For example, one female appeared to be extremely careless with her baby. Instead of holding it gently, she either clasped it roughly and pressed it tightly to her body, or placed it beside her on the rocks - a very unusual method of treating a newborn monkey. Just before it died, the young animal could be seen gasping for breath as its mother squeezed its chest. (222 f.)

Diese Kurzdarstellung offenbart durch die narrative Darstellung und die dramatisierte Emphase auf das Leidensbild im letzten Satz des zweiten Zitates einen Hang zur Erzählung als Vergegenwärtigung der Forschungserfahrung. Durch die an den szientistischen Diskurs anknüpfenden Wissenschaftlichkeitssignale (z. B. „Observations made“, ,There is a record“) wird diese Kurzdarstellung des häufigsten Kindstods unter den Pavianen jedoch scheinbar firm in den Kontext der Überblicksdarstellung zum Monkey Hill eingeordnet.

Drittens erlaubt es das derart vermittelte Fundament der Beobachtungen und der statistischen Übersicht, die hier kreiert wird, Zuckerman, einen gesellschaftstheoretischen - also eigentlich jenseits seiner Expertise liegenden - Schluss zu ziehen: 
The number of fatal fights that have followed deaths on the Hill is too great to be without significance, and the meaning of the correlation is obvious. The equilibrium of a social group is dependent upon the mutual reactions of all its members. The death of any single individual upsets the state of balance, and fighting commonly breaks out before a new equilibrium is reached. (222)

Es ist ausgerechnet diese, von seinem anatomisch-physiologischen Programm am weitesten entfernte soziopolitische Betrachtungsweise der Forschungsergebnisse des Monkey-Hill-Experiments, die Zuckerman am ehesten anschlussfähig für die spätere primatologische Ethologie werden lassen. ${ }^{45}$

Zuckermans Forschungs- und Darstellungsprogramm erfüllt sich in The Social Life of Monkeys and Apes aber nur bedingt, gleicht der Text doch bei näherer Betrachtung von Zuckermans Forschungsgrundlage, vor allem des Sozialexperiments Monkey Hill, selbst jener ,mêlée', als die er gern die meist fatalen Kämpfe unter den Pavianen bezeichnet. Zwischen der Wiedergabe von altem Affenwissen aus dem 17. bis 19. Jahrhundert, der Zusammentragung von Versuchen anderer, den eigenen Beobachtungen und Spekulationen, sowie der Verachtung für jene eingehende Langzeitforschung, in situ', die ein wichtiges Fundament der modernen Primatologie werden sollte, ist The Social Life ein heterogener Text. Allerdings versieht Zuckerman ihn an seinen Kernstellen mit einem szientistischen Darstellungsstil, der seinen programmatischen Forschungsansatz ex post hervorzubringen scheint. Dieses ,Programm ' fungiert daher eher als ein Anspruch, der sich in der Folge der Publikation in Zuckerman als, wie sein Biograph Donovan ihn im Untertitel nennt, „scientist extraordinary“ verwirklicht. Die deterministische Perspektive des Anatoms, der Verhalten als Ergebnis oder Ausdruck physiologischer Vorgänge behandelt, die durch experimentelle Analyse hervorgebracht werden können, führt ihn zu kriegsrelevanter Schädeltraumata-Forschung, lässt ihn zum wissenschaftlichen Berater der Politik und zur - durchaus auch ambivalenten - Ausnahmefigur im Wissenschaftsbetrieb werden. Die primatologische Gemeinde betrachtet die Ergebnisse seiner Forschungsarbeit zum Sozialverhalten und zur Reproduktion von Affen mittlerweile jedoch größtenteils als unbedeutend, wenn nicht sogar falsch. Die Primatologin Allison Jolly nennt Zuckermans Ansatz beispielsweise so schlicht wie harsch „representative of the undeveloped state of scientific knowledge and methodology in his era". ${ }^{46}$

\subsubsection{Navigating Science: Emotionsregime und Gefühlsregeln}

Was sowohl Köhlers Intelligenzprüfungen an Anthropoiden als auch Zuckermans The Social Life of Monkeys and Apes zeigen, ist nicht nur eine Durchdringung des Textes mit den wissenschaftspolitischen Normen ihrer Zeit. Sie thematisieren auch

\footnotetext{
${ }^{45} \mathrm{Vgl}$. Strum/Fedigan: Changing Views of Primate Society, S. $41 \mathrm{ff}$.

${ }^{46}$ Jolly: The Bad Old Days of Primatology, S. 9.
} 
den darin spezifischen Stand emotional konnotierter anthropomorphisierender und narrativ arbeitender Interpretations- und Darstellungsverfahren. Beide Texte zeigen, dass der Anthropomorphismus- ebenso wie der Anekdoten-Verdacht wirkmächtige Probleme sind, von denen sich ein wissenschaftlicher Autor zeitgenössisch abzugrenzen hat. Meines Erachtens ist diese Abgrenzung jedoch eine taktische Umgehung. Wissenschaftliche Texte reagieren strategisch auf das Problem der abgewerteten Emotionalität in der Forschung. ${ }^{47}$

In seiner emotionshistorischen Studie The Navigation of Feeling entwirft William Reddy das Konzept von „emotional regimes“. ${ }^{48}$ Prämisse ist für Reddy, dass Emotionen „largely (but not entirely) learned“49 und die geschichtliche Vergangenheit ,a record of human efforts to conceptualize our emotional makeup, and to realize social and political orders attuned to its nature "50 seien. Als ,the most immediate, the most self-evident, and the most relevant of our orientations toward life" 51 spielen Emotionen, so Reddy, eine zentrale Rolle im sozialen Leben. Aufgrund dieser Zentralität bedürfen Emotionen einer sozialen Bestimmung und Regulierung, um den Ethos und die Stabilität einer Gesellschaft zu gewährleisten. Reddy nimmt für alle historischen Gesellschaften an, dass sie erstens Emotionen als einen wichtigen Bereich bzw. eine Leistung konstruieren (,an important domain or effort ${ }^{\text {"552) }}$ und dass sie zweitens Individuen mit ,prescriptions and counsel concerning both the best strategies for pursuing emotional learning and the proper end point or ideal of emotional equilibrium" "53 versehen. Was Reddy somit als Emotionsregime beschreibt, ist ein historisch und kulturell variierendes, mehr

\footnotetext{
${ }^{47}$ Umso interessanter ist daher ihre Interpretation durch nicht-wissenschaftliche Instanzen. Köhlers Intelligenzprüfungen findet in Samuel Beckett einen aufmerksamen Interpreten. Dessen Acte Sans Parole I (1955), bzw. Act Without Words I (1956) wird von Köhring als Text gelesen, der „Wissen vom Tier und vom Theater als Wissen von Beobachtbarkeit und Äußerlichkeit im Spannungsfeld von Textualität und Performativität“ transponiere und ein Ort der Überkreuzung von Geschichten des Wissens sei. Köhring: Einsicht einsichtig werden lassen, S. 125. Der Acte bietet jedoch schlicht auch die Möglichkeit einer Aufführung des emotionalen Gehaltes der von Köhler beschriebenen Szenen. Köhlers Text thematisiert (s. o.) die tierliche Emotionalität rund um das Experiment; Becketts Pantomime verdeutlich mit menschlichen Darstellern die tierliche Emotionalität als Gegenstand des Experimentierens. Beckett, Samuel: Act Without Words I. In: Ders.: Collected Short Plays. London 1984, S. 41-46.

${ }^{48}$ Reddy, William M.: The Navigation of Feeling: A Framework for the History of Emotions. New York 2001, S. xiii.

${ }^{49}$ Reddy: The Navigation of Feeling, S. xi.

${ }^{50}$ Reddy: The Navigation of Feeling, S. xii.

${ }^{51}$ Reddy: The Navigation of Feeling, S. 3.

${ }^{52}$ Reddy: The Navigation of Feeling, S. 55, Hervorheb. im Original.

${ }^{53}$ Reddy: The Navigation of Feeling, S. 55, Hervorheb. im Original.
} 
oder minder bewusstes ,collective shaping of emotional effort and collective elaboration of emotional ideals “54 im Dienste politischer Stabilität. ${ }^{55}$

Arlie Russell Hochschild diskutiert bereits Ende der 1970er Jahre den engen Zusammenhang von sozialer Ordnung und emotionalem Erleben, genauer: die Verbindungen von ,social structure, feeling rules, emotion management, and emotive experience“. ${ }^{56}$ Hochschild definiert in Bezug auf (gesamt-)gesellschaftliche und Klassen-Konventionen des Umgangs mit Emotionen feeling rules als ,the side of ideology that deals with emotion and feeling" und emotion management als ,the type of work it takes to cope with feeling rules. " ${ }^{\text {"57 }}$ Die Gefühlsregeln als sozial geteilte, jedoch meist latente Richtlinien bestimmen laut Hochschild das Ausmaß, die Richtung und die Dauer von Gefühlen. Daher lässt sich - so greift Hochschild hier Reddy vor - von den ,rights and duties of feeling “58 sowohl auf die Reichweite sozialer Konventionen und Kontrolle als auch auf die Zugehörigkeit des/der Einzelnen zu bestimmten sozialen Kreisen schließen.

Dass Hochschilds Konzepte zur Thematisierung von Emotionalität als Teil menschlicher Arbeitskapazität auch im 21. Jahrhundert noch maßgeblich sind, zeigt sich beispielsweise daran, dass die Kulturanthropologin Gertraud Koch sie

\footnotetext{
${ }^{54}$ Reddy: The Navigation of Feeling, S. 56.

${ }^{55}$ Reddys Studie widmet sich einer für das einzelne Individuum relevanten ,ppolitics of emotions" (Reddy: The Navigation of Feeling, S. 114), welche sich in eben jenen Regelungs- und Ordnungssystemen ausdrückt, die Reddy unter dem Begriff der Emotionsregime fasst. Dabei sollen, so Reddy, die Ergebnisse sozialkonstruktivistischer Emotionsforschung um jene der kognitiven Psychologie ergänzt werden. Instrument für diese Überwindung des vermeintlichen Grabens zwischen den beiden Ansätzen Relativismus und Universalismus - ist ihm John Austins Sprechakttheorie. Zwischen den beschreibenden und abbildenden Aussagen über die Welt (constatives) und den weltverändernden Aussagen (performatives) siedelt Reddy Gefühlsaussagen (emotives) an, die sowohl weltbeschreibende als auch weltverändernde Eigenschaften besitzen. Vor allem beherbergen diese Sprechakte mehr als eine Doppelfunktion: Nicht nur sind sie potenziell weltbeschreibend und -verändernd zugleich, sie können auch Teil der „strong emotional management tools" (S. 122) eines Emotionsregimes sein und Individuen eine Navigation durch die unterschiedlichen widersprüchlichen Zielorientierungen von Emotionen ermöglichen. Diese emotives bilden daher den Grundbaustein von Reddys Theorie der Emotionsregime. Vgl. S. 96 ff.; Plamper: Geschichte und Gefühl, S. 297 ff.

${ }^{56}$ Hochschild: Emotion Work, S. 551.

${ }^{57}$ Hochschild: Emotion Work, S. 551. In ihrem für die Emotionssoziologie prägenden Aufsatz aus dem Jahr 1979 konzeptualisiert Hochschild in der Auseinandersetzung mit Erwin Goffman und Sigmund Freud eine ,human capacity for, if not the actual habit of, reflecting on and shaping inner feelings, a habit itself distributed variously across time, age, class, and locale" (S. 557). Später fügt Hochschild dieser Aufzählung noch das Geschlecht bei, wenn sie spezifisch Gefühlsarbeit in ,weiblichen' Berufen untersucht. Siehe Hochschild: Das gekaufte Herz.

${ }^{58}$ Hochschild: Emotion Work, S. 564.
} 
umfassend übernimmt und feeling rules als zentrales Element in und für emotional labor in spezifischen Arbeitskulturen definiert: ${ }^{59}$

Erst wenn implizite oder explizite Gefühlsregeln existieren, wird Emotionsarbeit, also die Anpassung des eigenen Fühlens an die sozial erwartete Emotionslage notwendig. Die Diskrepanz zwischen den eigenen Gefühlen und den sozialen Erwartungen wird in der sozialen Interaktion wahrgenommen. Solche Abweichungen sind für das Individuum Anlass, das eigene Empfinden zu reflektieren und gegebenenfalls das eigene Fühlen mit den Normen in Einklang zu bringen $[\ldots] .{ }^{60}$

Gefühlsregeln sind daher auch bei Koch „ein Modus des Normierens, das in sozialer Interaktion seine Wirksamkeit erlangt“; 61 sie sind (mit Hochschild) „the ,bottom side" of ideology" 62 indem sie die in Weltanschauungen eingelassenen sozialen Ordnungen ausdrücken und bis ins Individuum hinein materialisieren:

Gefühlsregeln stellen das Bindeglied zwischen individuellem Erleben und sozialem Kontext her. Sie spielen eine wesentliche Rolle bei der Sozialisation in die Regeln und Normen einer sozialen Gruppe und auch in Organisationen. Gefühlsregeln sind dabei Ausdruck von Weltanschauungen dieser Gruppe. Erlebte Gefühle können so in ganz unterschiedlichen weltanschaulichen Rahmen erfahren werden, die innerhalb einer Gesellschaft verfügbar sind, etwa als geschlechtsspezifische Gefühlsregeln oder als professionelle Gefühlsregeln. ${ }^{63}$

Obwohl damit unterschiedliche Repertoires an Gefühlsregeln für das Individuum und für die Interpretation des eigenen emotionalen Erlebens zur Verfügung stehen, wird die vermeintliche Freiheit von Handlungsspielräumen ,zwischen den Rahmen' durch framing rules eingeschränkt, die priorisieren, ,welcher Rahmen in einer Situation der akzeptiertere bzw. der wichtigere ist“, und die „die sozialen Ordnungen sowie die Wissensordnungen wider[spiegeln], innerhalb derer sich Gefühlsregeln und weltanschauliche Rahmen verorten." ${ }^{64}$

In der Primatologie scheinen mir deutlich die von Reddy konzipierten Emotionsregime und jene von Hochschild und Koch identifizierten Gefühlsregeln

\footnotetext{
${ }^{59}$ Koch, Gertraud: ,Feeling Rules ${ }^{\text {- }}$ Eine Theorieperspektive zur Erforschung von Arbeitskulturen. In: Manfred Seifert (Hg.): Die mentale Seite der Ökonomie. Gefühl und Empathie im Arbeitsleben. Dresden 2014, S. 51-69, hier S. 51 f. Zum emotional labor-Konzept siehe auch Lutz, Catherine: Feminist Theories and the Science of Emotion. In: Frank Biess/Daniel M. Gross (Hg.): Science and Emotions After 1945. A Transatlantic Perspective, Chicago/London 2014, S. 342-364, hier S. $353 \mathrm{ff}$.

${ }^{60}$ Koch: ,Feeling Rules', S. 53.

${ }^{61}$ Koch: ,Feeling Rules', S. 53.

${ }^{62}$ Hochschild: Emotion Work, S. 566.

${ }^{63}$ Koch: ,Feeling Rules', S. 59.

${ }^{64}$ Koch: ,Feeling Rules‘, S. 57. Bei Hochschild sind die framing rules ganz grundsätzlich die „rules according to which we ascribe definitions or meanings to situations“ und „the guidelines for the assessment of fits and misfits between feeling and situation". Hochschild: Emotion Work, S. 566. Wenn sich die (ideologisch bedingten) framing rules ändern, ändern sich auch die feeling rules für das Individuum.
} 
zusammenzutreffen: Wie Emotionalität in der Forschung von den Forschenden selbst wahrgenommen, genutzt, reflektiert und vor allem dargestellt werden kann, hängt von den jeweiligen historischen, kulturellen Emotionsregimen - bspw. den von Helmuth Lethen für die Weimarer Republik identifizierten , Verhaltenslehren der Kälte 65 oder jene um 1970 populäre Aufwertung von „Emotion as Authentic Feminity“66 - ebenso ab wie von den spezifischen Gefühlsregeln, die jeweils weltanschaulich und von Wissensordnungen abhängig für , die Wissenschaft ${ }^{6}$, das einzelne zum Bereich Primatologie beitragende Fach und/oder sein publizistisches Feld gelten. Gerade Reddys Hinweis, dass „,normative style of emotional management is a fundamental element of every political regime, of every cultural hegemony“, 67 lässt sich in den Bereich der wissenschaftlichen Emotionspolitik ausweiten. Auch hier gilt: „Leaders must display mastery of this style; those who fail to conform may be marginalized or severly sanctioned. In other cases, there may be a hierarchy of contrasting styles, failure to conform to one or another renders one's identity unclear, subject to exclusion“".68 Wie Lorraine Daston anmerkt, bedingt der Eintritt in eine wissenschaftliche ,Schule " nicht nur „ways of seeing, manipulating, and understanding“, sondern auch ,ways of feeling“. 69 So werden Denkkollektive (Fleck) als Gefühlskollektive (Daston) bzw. emotionale Gemeinschaften (Rosenwein) perpetuiert, die durchaus auch in der weitgehenden Ablehnung von Emotionalität ihre emotionale Vergemeinschaftung (Plamper) finden können. ${ }^{70}$ Wie die Ergebnisse primatologischer Forschung, die

\footnotetext{
${ }^{65}$ Lethen: Verhaltenslehren der Kälte.

${ }^{66}$ Lutz: Feminist Theories, S. 348.

${ }^{67}$ Reddy: The Navigation of Feeling, S. 121.

${ }^{68}$ Reddy: The Navigation of Feeling, S. 121. Dass das ,Entanglement‘ von Forschungsemotionen und wissenschaftlichen Denkstilen kein neuer Untersuchungsgegenstand ist, zeigt Uffa Jensens Diskussion von Ludwik Flecks bereits in der Einleitung der vorliegenden Studie (Abschn. 1.2) angesprochenem Entstehung und Entwicklung einer wissenschaftlichen Tatsache (1935). Jensen fasst Flecks Untersuchung der epistemologischen Situation einer Entdeckung wie folgt: „researchers who observe a phenomen that contradicts the conventional wisdom in their area of expertise enter a state of emotional chaos. To overcome this state, Fleck tells us, they try to remain faithful to the common thought style and thought collective. Emotional tranquility can be reestablished only if they find an explanation for the observed phenomenon in accordance with the prevalent thought style. If this fails, the uncertainty and anxiety will persist until a new explanation in a novel thought style is found“. Jensen: Across Different Cultures?, S. 264.

${ }^{69}$ Daston: The Moral Economy of Science, S. 5.

${ }^{70}$ Daston spricht mit Ludwik Flecks Terminologie von den wissenschaftlichen Denkkollektiven als Gefühlskollektiven. Vgl. Daston: The Moral Economy of Science, S. 5. Rosenwein entwirft als Alternative zu Johan Huizingas und Norbert Elias' Großnarrativen der zunehmenden Emotionskontrolle in der Moderne ,emotionale Gemeinschaften' als Gruppen von Menschen, die Normen des Gefühlsausdrucks ebenso teilen wie eine Bewertung von Gefühlen. Es kann sich dabei um durch Nahverhältnisse konstituierte Gemeinschaften ebenso handeln wie um Text-, Arbeits-, Wohn-, oder Forschungsgemeinschaften. Individuen sind grundsätzlich in der Lage, zwischen diesen emotionalen Gemeinschaften - z. B. der Familie und der Arbeitswelt zu wählen bzw. zu wechseln und ihr emotionales Verhalten jeweils anzupassen. Vgl. Rosenwein: Worrying about Emotions; Rosenwein, Barbara H.: Emotional communities in the early Middle Ages. Ithaca 2006; Plamper: Geschichte und Gefühl, S. 78 ff.
} 
Forschungsarbeit selbst und die Forschenden-Persönlichkeit in ihrem wissenschaftlichen Feld - und darüber hinaus - wahrgenommen werden, hängt dann nicht nur wesentlich davon ab, welche Publikationsform - z. B. wissenschaftliche/r Aufsatz oder Monographie, National-Geographic-Titelgeschichte oder Forschungsmemoiren -, sondern auch, welcher (je nach Form, Disziplin und Theorierichtung) normative Stil des Emotionsmanagements gewählt wird.

Bislang wurde in dieser Arbeit untersucht, welchen Stellenwert Emotionalität in den Forschungs- und Wahrnehmungsprozessen der primatologischen Feldarbeit hat (Affektive Epistemologien) und wie diese als literarische bzw. Textformen in den Forschungsmemoiren zum Ausdruck kommen (Affektpoetik). Nun soll im Folgenden der Blick auf Textstrategien gelenkt werden, in denen sich Emotionsregime und Gefühlsregeln ausdrücken: Geht man davon aus, dass, wie diskutiert, bestimmte Formen der Emotionalität entweder Begleiterscheinungen - das Erhabene oder die Rührung - oder sogar notwendig - soziale Emotionen, Empathie - für die Feldethologie sind (die Prämisse der im weitesten Sinne, epistemologischen Emotionen '), so lässt sich vergleichen, ob und wie Emotionalität in Texten zur Sprache kommt, die anderen Emotionsregimen und Gefühlsregeln unterliegen als die Episteme der ,Trimates', die den Schwerpunkt der bislang untersuchten Literatur bildete.

Nach und bereits während der Feldforschungs-Pionierzeit der ,Trimates' hat Emotionalität aufgrund ihrer engen Assoziation mit wissenschaftlichem Laientum, Geschlechterpolitik und Öffentlichkeitswirksamkeit einen schweren Stand in der Darstellung primatologischer Langzeitforschung. Die Gründe hierfür sind, wie bereits eingangs diskutiert, historisch und vielzählig. Frank Biess und Daniel M. Gross führen in ihrer Einleitung zum Sammelband Science and Emotion After 1945 die Marginalisierung von Emotionen und den ,,[re]turn to rationalism in postwar social thought" ${ }^{\star 71}$ auf ein „intense entanglement with emotions across a variety of disciplines"72 im späten 19. und frühen 20. Jahrhundert zurück. Denn die in dieser langen Jahrhundertwende aufblühende ,Entdeckung" und Thematisierung von Emotionen (etwas William James' „What is an Emotion“ von $1884)^{73}$ stehe im Kontext der Konzeption eines ,hydraulischen ' Emotionsmodells, das Emotionalität im Bild einer Art zum Ausbruch bereiten und im Zuge moderner Selbstbeherrschung zu unterdrückenden Magmas entwirft. ${ }^{74}$ Die zudem im transatlantischen politischen Diskurs hergestellte Verbindung von Emotionalität, Irrationalität und Faschismus lässt das „narrative of modernity“ laut Biess und

\footnotetext{
${ }^{71}$ Biess, Frank/Gross, Daniel M.: Emotional Returns. In: Dies. (Hg.): Science and Emotions After 1945. A Transatlantic Perspective. Chicago/London 2014, S. 1-38, hier S. 4.

${ }^{72}$ Biess/Gross: Emotional Returns, S. 5.

${ }^{73}$ James, William: What is an Emotion? In: Mind 9/34 (1884), S. 188-205.

${ }^{74}$ Barbara Rosenwein verknüpft dieses u. a. aus der mittelalterlichen Ausprägung der antiken Säftelehre und Lebensenergie-Vorstellungen gespeiste ,hydraulische ' Emotionsmodell mit Huizinga, Febvre, Bloch und Elias, aber auch Darwin und Freud. Vgl. Rosenwein: Worrying about Emotions, S. $834 \mathrm{ff}$.
} 
Gross identisch werden mit ,the development of increasingly refined practices of individual and collective emotional restraint and (self-)control“ ". ${ }^{75}$ Der Ruf der Emotionen als das ,Andere der Vernunft ${ }^{6}$ ist damit weiter gefestigt. ${ }^{76}$ Zwar treten ab den 1960er Jahren Emotionen graduell wieder als Gegenstand wissenschaftlicher Forschung in den Fokus, das Rationalitätsgebot des wissenschaftlichen Diskurses selbst formiert jedoch weiter diese Art von entemotionalisierenden ,practices of restraint ' für die Forschungspraxis und den Diskurs selbst. ${ }^{77}$ Erst die Konsolidierung des in den Sozial- und Geisteswissenschaften bereits u. a. durch Martha Nussbaums und ethnologische Arbeiten virulenten ,Emotional Turn\% ,Affective Turn' auch in den Neurowissenschaften öffnet die Emotionsforschung wieder für die ,wissenschaftlich“ legitimierte Thematisierung der Beteiligung von Emotionalität an kognitiven und epistemischen Prozessen. ${ }^{78}$ Diese ist jedoch sehr erfolgreich: Patrick Becker spricht vor dem Hintergrund einer Einbettung dieser Entwicklung in einen soziokulturellen Kontext so kritisch wie zeitdiagnostisch von einer „umfassenden Hinwendung zum Emotionalen“, die sich eben auch in den Wissenschaften als „Emotionalisierung der Vernunft“79 bemerkbar mache.

Anders als die ,Trimates“ Jane Goodall, Dian Fossey und Biruté Galdikas, die sich letztlich mit der Wendung hin zur Lebensaufgabe des Artenschutzes und der Abwendung von der wissenschaftlichen Forschungsarbeit die Lizenz zu Emotionalität im Text erworben haben (überwiegend um den Preis wissenschaftlicher Kredibilität), müssen Autor/innen, die dem Wissenschaftsbetrieb weiter erfolgreich angehören wollen, in ihrem Berichten von der Forschungsarbeit den wissenschaftlichen Emotionsregimen und ihren Gefühlsregeln folgen und in diesem Sinne zwischen den Erinnerungen an ihr emotionales Erleben und der Darstellung der Forschungserfahrung ein emotionales Management einkehren lassen.

Im Folgenden sollen drei Fälle untersucht werden, die dies illustrieren: Erstens ein neues, gesellschaftlich fundiertes theoretisches Paradigma, das so weit in die Interpretation, aber auch die Darstellung der Forschung hineinreicht, dass Emotionen bei Mensch und Tier zunächst weitgehend aus dem Blick geraden - nur um dann unter anderen Vorzeichen wiederzukehren (4.2 Neoliberale Gene); zweitens ein Modus, der im Einklang mit dem ,post-ironischen' Emotionsregime um

\footnotetext{
${ }^{75}$ Biess/Gross: Emotional Returns, S. 5.

${ }^{76}$ Zur zentralen Rolle, die Affektkontrolle in den soziologischen Erklärungsmodellen der Moderne bei Max Weber und Norbert Elias spielt, siehe Becker: Rationalisierung des Gefühls, S. $65 \mathrm{f}$.

${ }^{77}$ Plamper grenzt jedoch die ab den 1960ern in Erscheinung tretende sozialkonstruktivistisch pluralistische Emotionsethnologie, die die auch epistemologisch motivierte Gefühls-Selbstreflexion der gegenwärtigen Ethnologie hervorbringt (vgl. Abschn. 1.2), von einem die universalistisch angelegte neurowissenschaftliche Emotionsforschung der Gegenwart vorbereitendem psychologischen Emotionsboom ab. Damit zeigt er die Vielfalt der meist konkurrierenden Ansätze einer solchen Entwicklung hin zu Emotionen als Forschungsgegenstand auf. Vgl. Plamper: Geschichte und Gefühl, S. 87 ff., 239 ff.

${ }^{78}$ Vgl. Biess/Gross: Emotional Returns, S. 8 f.

${ }^{79}$ Becker: Rationalisierung des Gefühls, S. 64.
} 
2000 unter dem Vorzeichen der Ironie Emotionalität zulässt, aber zugleich gegen ihren schlechten Ruf immunisiert (4.3 Darting the Inner Prehistoric); und drittens neue Forschungspraktiken, die die Möglichkeiten affektiver Erfahrungen am Tier reduzieren, und damit auch das Schreiben von der Feldforschung modifizieren (4.4 Von deutscher Art und Aff?). Abschließend soll anhand zweier Romane ein Blick auf die Gegenwartsliteratur geworfen werden, wie sie in den letzten Jahren zunehmend die ethische Dimension von Forschungsemotionalität im Zeichen verschiedener Gefühlskulturen und Regime der Wissenschaft(lichkeit) thematisiert (4.5 In der Gegenwart).

\subsection{Neoliberale Gene: Primatologie im Zeichen der Ökonomie}

William Reddy grenzt in The Navigation of Feeling exemplarisch am Fall Frankreichs ein Emotionsregime des frühen 19. Jahrhunderts - unter dem Stichwort ,Liberal Reason' - von jenem des späten 18. Jahrhunderts - unter dem Stichwort ,Sentimentalism‘ - ab. Erläuternd merkt Reddy an:

I am not claiming that the people of early nineteenth-century France were without emotions, nor that they were without a past. It would have been impossible to completely eliminate the intellectual legacy of the sentimentalist period, just as it would have been impossible to recast personal, familial, and public practices in a form completely devoid of sentimentalist features. But this legacy and these features of practice were set in a new intellectual framework and put to new uses. ${ }^{80}$

Mit einem ähnlichen Verständnis möchte ich im Folgenden den Blick von der Episteme der ,Trimates ‘ und ihren Zeitgenoss/innen auf eine nachfolgende Generation primatologischer Feldforschender wenden, die in den 1970er und 1980er Jahren ihre ersten Feldstudien durchführten bzw. veröffentlichten. Zeigte sich trotz der unterschiedlichen Publikationsdaten in den Schriften der älteren Generation eine forschungsrelevante Wertschätzung von und reflektierende Auseinandersetzung mit Emotionalität in der Forschungspraxis, so gilt es nun zu analysieren, wie sich nachfolgende Autor/innen zum Feld ,Emotionalität und Wissenschaft" in einer neuen Episteme - in der neue Theorien und Modelle in der Forschung, neue politische und ökonomische Ansätze in Wirtschaft und Politik und neue gesellschaftsund kulturtheoretische Probleme in der Diskussion zusammentreffen - verhalten. Dazu werden in diesem Kapitel exemplarisch zwei Qualifikationsschriften untersucht: Sarah Blaffer Hrdys The Langurs of Abu (1977) und Barbara Smuts' Sex and Friendship in Baboons (1985). Dabei soll argumentiert werden, dass die Art und Weise, wie Emotionalität in den beiden Texten zum Tragen kommt, nicht allein mit den Gefühlsregeln wissenschaftlicher Denkkollektive, sondern auch mit dem Stellenwert von Emotionen in einem ökonomischen Regime zu tun hat.

\footnotetext{
${ }^{80}$ Reddy: The Navigation of Feeling, S. 217, Hervorheb. MS.
} 


\subsubsection{Sarah Blaffer Hrdys The Langurs of Abu (1977)}

\subsubsection{Regime der Theorie (1)}

Sarah Blaffer Hrdy beginnt den auf ihren Dissertationsstudien beruhenden Forschungsbericht The Langurs of $A b u$ ähnlich wie ihre Vorgänger/innen mit Informationen über den Forschungsgegenstand. Die Biogeographie und die Evolution ebenso wie der Name der Hanuman-Languren (Presbytis entellus) wird erläutert, und die Geschichte ihrer Erforschung seit dem 19. Jahrhundert anhand längerer Zitate aus historischen Quellen reflektiert (3 ff.; 54 ff.). Es geht in diesen ersten Kapiteln auch bereits um methodische Fragen: erstens vor allem um den Umgang mit ,anekdotischem ' Material, dessen Wert in diesem Fall, so Hrdy, von der frühen Ethologie unterschätzt wurde; und zweitens um die Abgrenzung von Alfred Radcliff-Browns Ansatz der späten 1950er Jahre, in dem „behaviour was explained in terms of group survival“ (7). Hrdy steuert hier auf ihren zentralen Untersuchungsgegenstand - ,infant killings` - zu und legt dar, dass dieser Forschungsgegenstand in ältere, auf die Spezies oder Gruppe konzentrierte Erklärungsansätze, bspw. die von Konrad Lorenz propagierte Tötungshemmung innerhalb der Spezies, nicht integriert werden konnte und daher lange als abnormes Verhalten eingeschätzt und ignoriert wurde. Erst jüngere Studien der 1960er Jahre konnten die Eindrücke früher ,anekdotischer' Berichte von Reisenden replizieren und feststellen, dass Infantizid ein zu häufig zu beobachtendes Verhalten unter Languren ist, um nur durch Überpopulation hervorgerufen zu sein und nicht einer Verhaltensnorm zu entsprechen.

Hrdy benennt ihr Forschungsziel - „to test the hypothesis that crowding was responsible for infant killing among langurs“ - und den Zeitrahmen (1971-1975), in dem sie diesem nachging. In diesem Zeitrahmen, so führt sie zugleich aus, kam es zu einer Umwälzung des theoretischen Wissens:

By the time I concluded my research [...] I had re-learned what a few British naturalists had known almost a century before: that langur males compete fiercely for possession of females, and that in the process, conspecifics are sometimes killed. [...] Far from being maladaptive, infanticide was found to be a widespread adaption to normal conditions of langur life that was quite advantageous to those males who practiced it. (10)

Hrdy scheint zu gleichen Teilen daran interessiert, den Grund für dieses Verhalten zu erforschen, wie der Frage nachzugehen, „,why we had for so long chosen to regard these incidents as unnatural or pathological behavior" (10). Diese Frage führt Hrdy wieder zurück zur theoretischen Grundlage der Ethologie. Als Grund für den „langur whitewash“ (10) sieht sie eine ,anthropocentric vein“: „Because we are human and hold ourselves to be unique, we tend to value highly our own ideals“ (10). Dies führe zu einer Projektion von „species survival“ (10) und „group maintenance“ (10) auf andere Primaten. Im Umkehrschluss könne eine hier propagierte kritische Auseinandersetzung mit theoretischen Vorannahmen in der Tierethologie auch auf das Menschenbild rückwirken: „By revealing our misconception about other primates, the langur saga may unmask misconceptions about ourselves“ (11). Sarah Blaffer Hrdy setzt somit in den Präliminarien 
ihres Forschungsberichtes bereits einen Schwerpunkt auf die Auseinandersetzung mit theoretischen Grundlagen für die Schlüsse, welche sich aus der Verhaltensbeobachtung im Feld ziehen lassen. Darin unterscheidet sie sich größtenteils von den Pionier/innen der Feldforschung, denen die Einrichtung der Feldforschung als Methode, die Erschließung von Tiergruppen und ihren Habitaten und die Grundlagenforschung oblag. Hrdy dagegen kann sich für ihr Feld - die Languren-Forschung - bereits auf zwei grundlegende Studien beziehen und sich von deren Ansätzen zum Teil abgrenzen. ${ }^{81}$

Basierend auf den bereits erfolgten und ausgewerteten Studien und eigenen Beobachtungen kann Hrdy konstatieren, dass ,the political organization and composition of each troop is constantly changing“, dass sich aber ein ,hypothetical model of langur social organization" (36) erstellen und durch ihre Beobachtungen in Abu illustrieren lasse. Es handelt sich um ein zyklisches, mehrphasiges Modell: „In the initial stage, a male from outside the troop usurps control of a bisexual troop. He evicts all other adult and subadult males and kills unweaned infants“ (36, Hervorheb. im Original). Es folge eine growth stage, in der Weibchen schwanger werden und Nachwuchs gebären, und eine mature stage, ,when after five years or more all age grades are represented“ (37). Dieses Stadium der Gruppe kann jedoch durch erneute ,male take-overs“ verhindert werden, „returning the troop to the initial stage of the cycle“" (37). Nachdem Hrdy für ihr nicht-disziplinäres Publikum einige Definitionen aufblättert - , home range', ,multimale troop', , onemale troop', ,alpha male ' und ,alpha female', ,extratroop males', , all-male bands' ,invader' und ,alien“ (44f.) - kommt sie zu Ausführungen, die auf das Gerüst ihres zugrunde liegenden Theoriegebäudes verweisen. Unter dem Zwischentitel „Genetic Consequences of Langur Troop Structure“ (46) wird die Sozialstruktur der Langurengruppen an genetische Beziehungen und Vorteile gebunden. Dabei benennt Hrdy das Personal ihrer theoretischen Bezüge: Charles Darwin, Ronald Aylmer Fisher, George Charles Williams und vor allem Robert Trivers. Diese Abfolge ist wichtig, denn sie zementiert das soziobiologische Theoriegebäude, in dem Hrdy ihren Forschungsbericht spielen lässt. Charles Darwins Theorie der sexuellen Selektion, die „differential success“ als „consequence of competition between members of one sex for access to the other sex" (48) umschreibt, wird in ihrer Relevanz durch die Arbeiten des Genetikers Ronald Aylmer Fisher im ersten Drittel des 20. Jahrhunderts bestärkt. ${ }^{82}$ Der Evolutionsbiologe George Charles

\footnotetext{
${ }^{81}$ Dies ist vor allem „Phyllis Jay’s pioneer research at the forest of Orcha“ (LA, S. 27) von 1958-59 und Yukimaru Sugiyamas mit vier anderen Mitgliedern des Japan India Joint Project ausgeführte Studie aus den Jahren 1961-62 (vgl. LA, S. 8). Wie Hrdy anhand mehrerer Tabellen aufzeigen kann, ist ihre Studie eine von 11 in den Jahren 1958-1975 an unterschiedlichen Forschungsorten durchgeführten Languren-Studien (vgl. S. 28 f., 32 f., 34 f.). Ihre eigene Forschungsarbeit findet in den Jahren 1971-1975 an der Forschungsstation Mount Abu statt. Insgesamt verbringen Hrdy und ihr Ehemann 11 Monate im Feld (vgl. S. 28 f., 36).

${ }^{82} \mathrm{Vgl}$. Darwin: On the Origin of Species und z. B. Fisher, Ronald Aylmer: The Genetical Theory of Natural Selection [1930]. New York 1958.
} 
Williams baut das Konzept in den 1960er Jahren bis auf die Ebene der Gene hinunter weiter aus und begründet die Theorie einer Gen-zentrierten Evolution, wie sie vor allem von Richard Dawkins in seinem The Selfish Gene (1976) populär gemacht wurde. Robert Trivers schließlich verfeinert diesen Ansatz Anfang der 1970er, indem er, wie seine Schülerin Sarah Blaffer Hrdy es formuliert, „the key variable determining the extent of intrasexual competition“ hervorhebt: „the relative amount of parental investment by each sex“ (48). ${ }^{83}$ Die Idee des Elternaufwands und der damit verbundenen Vorstellung, ,that whichever sex's typical parental investment is greater will become the limiting resource for the other sex " (48), prägt die Evolutionsbiologie fortan, indem sie ein grundlegendes Modell vorgibt: „Individuals of the sex investing less in an offspring will compete among themselves for the sex investing more“ (48). Für die meisten Säugetiere, so hebt Hrdy hervor, gilt: Weibchen, limitiert durch ,a definite physiological ceiling on the number of offspring they can produce and rear in a lifetime“ (49), müssen mehr in den Fortbestand ihrer Gene investieren als Männchen. In Hrdys Worten hat dies folgende Konsequenz:

Maximizing reproductive strategies for a female entails ensuring that any offspring she produces - already costly by the time they are born - survive, and minimizing the interval between births so that she comes as close as possible to the childbearing limit of which she is capable. A female's optimum strategy reflects some balance between these two policies. (49)

Um ihren Ansatz noch deutlicher zu machen, betont Hrdy:

Throughout this book, animals will be described as strategists, and it should be clear at the outset that no conscious calculations are necessarily implied. Rather, animals predisposed to respond to a given situation in particularly advantageous ways in the past contributed differentially to the next generation's gene pool. As used here, strategies are evolved, not invented. (49)

Das Thema von Hrdys Arbeit ist „the specifically female adaption to the loss of an infant which enables males to profit from killing infants in the first place" (49). Ich hebe hier diese Passage hervor, da sie in nuce und in deutlichen Worten nicht nur wiedergibt, auf welchem theoretischen Sockel Hrdys Forschungsarbeit steht - es geht um behaviorale Adaption im Sinne einer Gen-zentrierten Evolutionsbiologie -, sondern auch wie diese durch ihr Vokabular und ihre Prämissen die Forschungstiere als Akteure in einem bestimmten Sinn konzeptualisiert: Die Languren sind Strategen in einem Wettbewerb um die Gewinnmaximierung hinsichtlich des Fortbestehens der eigenen Gene im Genpool.

\footnotetext{
${ }^{83}$ Vgl. z. B. Trivers, Robert L.: Parental Investment and Sexual Selection. In: Bernard G. Campbell (Hg.): Sexual Selection and the Descent of Man, 1871-1971. Chicago 1972, S. 136-179; siehe auch Haraway: Primate Visions, S. 349 ff.
} 


\subsubsection{Das ökonomische Tier}

Damit konzipiert Hrdy, so die These, ein Tiermodell als Ausgangsbasis für ihre Arbeit über Languren, das mit der Idee eines homo oeconomicus korrespondiert. Der homo oeconomicus gilt seit dem späten 18. Jahrhundert als ein bzw. mit dem Siegeszug der Ökonomik als das Modell menschlichen Handelns. ${ }^{84}$ Er ist eine als rationaler Akteur und Nutzenmaximierer definierte Erklärungsfigur. In dem zugrunde liegenden Modell wird erstens das Individuum zur Grundeinheit der Analyse. ${ }^{85}$ Zweitens wird „menschliches Handeln als rationale Auswahl aus Alternativen" ${ }^{\text {"86 }}$ verstanden, so Gebhard Kirchgässner. Drittens geht man darin von einer Situation der Knappheit aus, „so daß er [der Mensch] nicht alle seine Bedürfnisse (gleichzeitig) befriedigen kann, sondern sich jeweils zwischen mehreren Möglichkeiten entscheiden muß“ ${ }^{87}$ Viertens handelt der Mensch in diesem Verhaltensmodell eigenständig entsprechend seinen Präferenzen. Kirchgässner nennt dies das ,Eigennutzaxiom“: „Das Individuum handelt (nur) entsprechend seinen eigenen Interessen“. .8 Im Alltagsverständnis impliziert der Begriff Rationalität zwar häufig Affektlosigkeit. Rationalität in diesem anthropologischen Modell bedeutet jedoch nur, „daß das Individuum prinzipiell in der Lage ist, gemäß seinem relativen Vorteil zu handeln, d. h. seine Handlungsspielraum abzuschätzen und zu bewerten, um dann entsprechend zu handeln" ${ }^{89}$ Die affektiven Implikationen eines solchen selbstzentrierten, interessegeleiteten Menschenbildes verfolgt auch Joseph Vogl bis in die Frühe Neuzeit hinein:

Zunächst wäre das Interesse ein letztes und unauflösliches Verhaltensatom. Was einer will oder begehrt, wohin ihn die Neigung oder Leidenschaft treibt - in jedem mehr oder weniger bewussten Entscheidungsprozess arbeitet eine Logik der Bevorzugung, an deren Ende stets das Bessere für einen selbst steht. [...] Damit zeigt sich das Interesse zugleich

\footnotetext{
${ }^{84}$ Der homo oeconomicus ist als Menschenbild zwar in Adam Smiths The Wealth of Nations (1776) erstmals vollständig modelliert. Es handele sich dabei jedoch nicht um eine revolutionäre Neuerfindung Smiths, sondern um eine „systematisierende Synthese bereits bekannter Theorien und Vorstellungen“ der Ökonomie und Moralphilosophie des 18. Jahrhunderts, die zudem tief „,in der Tradition scholastischer und merkantiler Ökonomievorstellungen" des späten Mittelalters und der Frühen Neuzeit verankert sei, so Volkmann, Laurenz: Homo oeconomicus. Studien zur Modellierung eines neuen Menschenbildes in der englischen Literatur vom Mittelalter bis zum 18. Jahrhundert. Heidelberg Winter 2003, S. 2.

${ }^{85}$ Kirchgässner legt in seiner Beschäftigung mit dem Verhaltensmodell des homo oeconomicus Wert darauf, dieses Modell in der Ökonomik als Methode zu verankern, nämlich als „Versuch, menschliches Verhalten dadurch zu erklären, daß man unterstellt, daß sich die einzelnen Individuen ,rational' verhalten. Individuen handeln dadurch, daß sie aus den ihnen zur Verfügung stehenden Möglichkeiten eine rationale Auswahl treffen“. Kirchgässner, Gebhard: Homo oeconomicus. Das ökonomische Modell individuellen Verhaltens und seine Anwendung in den Wirtschafts- und Sozialwissenschaften. Tübingen 1991, S. 2, Hervorheb. im Original. Dies sei als Analyseansatz dringend zu unterscheiden von der Ökonomie als Gegenstandsbereich der Wirtschaftswissenschaften, denn die Ökonomik ist für Kirchgässner eine Methode der Sozialwissenschaften.

${ }^{86}$ Kirchgässner: Homo oeconomicus, S. 12, Hervorheb. im Original.

${ }^{87}$ Kirchgässner: Homo oeconomicus, S. 12, Hervorheb. im Original.

${ }^{88}$ Kirchgässner: Homo oeconomicus, S. 16.

${ }^{89}$ Kirchgässner: Homo oeconomicus, S. 17.
} 
als Form des Wollens, das nicht über Askese, Selbstbeherrschung und Zügelung, sondern umgekehrt durch Selbstbehauptung funktioniert. Das Interesse kennt den Selbstverzicht nicht. Es fungiert als ein prinzipienloses Prinzip. ${ }^{90}$

Das Subjekt des Interesses ist bei Vogl eines, das Begehren, Neigung und Leidenschaften sehr wohl kennt. Rationalität bedeutet schlicht, dass es diese zum eigenen Vorteil nutzt und ihnen nicht ausgeliefert ist. Es ist dabei in Vogls Verständnis alles andere als ein Moral- oder Gesetzes-Subjekt, aber es ist ein notwendig sozial eingebettetes:

Wer aus Interesse handelt, kommt zwangsläufig zum Handel und Austausch mit anderen, er kommuniziert mit seinen Neigungen und bringt damit überhaupt soziale Gesetzmäßigkeiten hervor. Im Interesse begegnen sich die Neigungen und Leidenschaften aller Akteure, und gerade wo sie ihren Interessen folgen, offenbart sich schließlich ein soziales und politisches Naturgesetz. ${ }^{91}$

Richard Dawkins' Selfish-Gene-Variante der Evolutionstheorie verschiebt nun dieses selbstbezogene aber für Sozialformen konstitutive Interesse vom Individuum als Grundeinheit eines Verhaltensmodells eine Ebene tiefer auf jene der Gene. Für Dawkins sind menschliche und nicht-menschliche Tiere schlicht „survival machines robot vehicles blindly programmed to preserve the selfish molecules known as genes“.92 Dawkins' zentrales Argument ist, „that a predominant quality to be expected in a successful gene is ruthless selfishness. This gene selfishness will usually give rise to selfishness in individual behaviour": 93 „I shall argue that the fundamental unit of selection, and therefore of self-interest, is not the species, nor the group, nor even, strictly, the individual. It is the gene, the unit of heredity".94 Die dabei zugrunde liegende Analogie ist die des Computers. Die Gene stellen als ,,master-programmers “95 den ,Code ' her, der das Verhalten der biologischen Maschinen bestimmt, um im Sinne genau dieser Gene und ihrer Replikation bzw. Reproduktion zu handeln. Dabei übernimmt Dawkins das Verhaltensmodell der Ökonomik und erklärt es zugleich biologisch. ${ }^{96}$ Es ließe sich die Frage stellen, was zuerst da war: das Modell einer maximal eigennützigen, rational kalkulierenden Figur als kulturelles Paradigma oder die maximal eigennützigen, rational handeln lassenden Gene in Mensch wie Tier.

\footnotetext{
${ }^{90}$ Vogl, Joseph: Das Gespenst des Kapitals. Zürich 2010, S. 35 f.

${ }^{91}$ Vogel: Das Gespenst des Kapitals, S. 36. Auch bei Kirchgässner wird die soziale Einbettung des homo oeconomicus als seit Adam Smiths Wealth of Nations „fast trivial“ bezeichnet. Kirchgässner: Homo oeconomicus, S. 22.

${ }^{92}$ Dawkins, Richard: The Selfish Gene. Oxford/New York 1989, S. v.

${ }^{93}$ Dawkins: The Selfish Gene, S. 2.

${ }^{94}$ Dawkins: The Selfish Gene, S. 11.

${ }^{95}$ Dawkins: The Selfish Gene, S. 62.

${ }^{96}$ Kirchgässner weist jedoch darauf hin, dass die Vorstellung vom homo oeconomicus als „,vollständig informierte[r] und immer blitzschnell entscheidende[r] wandelnder Computer" nur ein unrealistischer Lehrbuchfall sei, sich also für eine Ökonomik, die realistische Prognosen erstellen wolle, nicht anbiete. Kirchgässner: Homo oeconomicus, S. 28.
} 


\subsubsection{Regime der Theorie (2)}

Hrdys Tiermodell speist sich aus dieser neuen Variante der Evolutionstheorie mit ihren Implikationen als Sozialbiologie. Auch wenn sie im Dienste einer anthropologischen Differenz versichert, dass für die Languren keine „,conscious calculations“ (49) angenommen werden müssen, sind die Tiere bei ihr als rationale Akteure beschrieben, die sich im Sinne ihres eigenen reproduktiven Vorteils bzw. im Interesse der Bevorteilung ihres eigenen Genmaterials - evolutionär programmiert - so verhalten, wie der homo oeconomicus im Dienste seines Interesses und letztlich seines genetisch-reproduktiven Vorteils handelt. Dass diese Übertragung von einem ökonomischen Verhaltensmodell menschlichen Handelns auf tierliches Verhalten möglich ist, liegt erstens daran, dass im Konzept des homo oeconomicus der philosophische Unterschied zwischen Verhalten und Handeln verschwindet: „Verhalten von Individuen wird erklärt, indem unterstellt wird, daß sie rational handeln“,97 wie Kirchgässner zusammenfasst. Denn nur so können ökonomische Analysen überhaupt zu Prognosen herangezogen werden. ${ }^{98}$ Zweitens wird in diesem ökonomischen Verhaltensmodell menschlichen Handelns bereits ein adaptives Muster angedeutet: Das Modell des homo oeconomicus unterstelle, so Kirchgässner, „daß sich Individuen an veränderte Umweltbedingungen entsprechend ihrer Zielvorstellungen (Präferenzen) in systematischer und damit vorhersagbarer Weise anpassen". ${ }^{99}$ Wie in der Ethologie geht es auch in der ökonomischen Theorie drittens trotz der Grundeinheit des Individuums weniger um das tatsächliche Verhalten einzelner Individuen, sondern um das ,typische“ Verhalten, d. h. die „Regelmäßigkeiten im Verhalten aller oder zumindest einer Mehrheit der betrachteten Individuen in der jeweils untersuchten Gruppe. “100

Das Verhältnis von mathematischer Ökonomie und Biologie ist traditionell eng. Beide sind ,evolutorische Wissenschaften', die mit analogen Interpretationen des von ihnen jeweils untersuchten Geschehens - ,Wettbewerb der Arten“ bzw. Entwicklung auf Wettbewerbsmärkten - arbeiten. Daher sind beide Wissenschaften besonders offen für gegenseitige Methoden-, Ideen- und Modelltransfers. ${ }^{101}$ Kirchgässner sieht in der Bereitschaft der Soziobiologie in den 1970er Jahren (Edward O. Wilson, Michael T. Ghiselin, David P. Barash, Richard Dawkins), die Biologie als vollständig ökonomische Disziplin zu denken, einen „,weiteren Schritt im Rahmen des ökonomischen Imperialismus“, ${ }^{102}$ der jedoch im Gegensatz zu anderen Bereichen, auf denen sich ökonomisches Denken ausgebreitet habe, von den Biologen selbst ausgeführt wurde. In letzter Konsequenz führe diese Formulierung der Biologie in einer Weise, die die menschliche Natur und die Grundlagen

\footnotetext{
${ }^{97}$ Kirchgässner: Homo oeconomicus, S. 17.

${ }^{98}$ Kirchgässner: Homo oeconomicus, S. $17 \mathrm{ff}$.

${ }^{99}$ Kirchgässner: Homo oeconomicus, S. 18.

${ }^{100}$ Kirchgässner: Homo oeconomicus, S. 21.

${ }^{101}$ Vgl. Kirchgässner: Homo oeconomicus, S. 215 ff.

${ }^{102}$ Kirchgässner: Homo oeconomicus, S. 225.
} 
der menschlichen Sozialorganisationen zu erklären beanspruche, sogar zu einem , biologischen Imperialismus ', der andere Wissenschaften in ihrer Eigenständigkeit bedrohe. ${ }^{103}$

An Sarah Blaffer Hrdys The Langurs of Abu zeigt sich aber nicht nur diese gegenseitige theoretische Durchlässigkeit von Biologie und Ökonomie, sondern auch ein Wandel der zugrunde liegenden ökonomischen Modelle. Das animal oeconomicum bei Hrdy ist nicht allein ein rationaler, eigennütziger Akteur, es ist ausgewiesenermaßen ein ,strategist". Hrdys Ethologie hat theoretisch nicht nur an einer neuen wissenschaftlichen Ausrichtung teil, sondern auch an einem neuen gesellschaftlichen Paradigma, das diese Ausrichtung erst hervorbringen kann. In der Sprache der zeitgenössischen Evolutionstheorie bildet sich der Einfluss mehrerer theoretischer Strömungen ab, die in der Nachkriegszeit an der Ausformung eines neuen politischen und ökonomischen Paradigmas ebenso wie an einer neuen wissenschaftlichen Episteme beteiligt sind. Dawkins' leitende, und zum Veröffentlichungszeitpunkt von The Selfish Gene originelle Analogie ist wie erwähnt zunächst die Maschine, bzw. deren raffinierteres Modell, der auf Rechenoperationen basierende Computer. Zugrunde liegt seiner Theorie eine älteres Modell der Evolution, das wettbewerbsbasiert (,competition') argumentiert und eine ökonomische Vorstellung von einer Abwägung von Kosten und Nutzen (,cost and benefit') beinhaltet, und ein noch älteres, in dem es um den Kampf ums Dasein geht (,battle', ,survival'). Mit der Aufnahme von Vokabeln wie ,strategy', ,investment' oder ,policy“ rückt ein neueres ökonomisches Modell in den Blick und es wird begleitet von den Vorstellungen einer spielerischen Spekulation (,gamble') um den optimalen Ausgang einer Strategie. Dawkins schreibt z. B.:

[A] reasonable policy for a female who is in danger of being deserted might be to walk out on the male before he walks out on her. This could pay her, even if she has invested more in the child than the male has. [...] A parent may be expected to desert, the moment it is possible for him or her to say the following: ,This child is now far enough developed that either of us could finish off rearing it on our own. Therefore it would pay me to desert now, provided I could be sure my partner would not desert as well. If I did desert now, my partner would do whatever is best for her/his genes. He/she would be forced into making a more drastic decision than I am making now, because I would have already left. [...] Therefore, assuming that my partner will take the decision that is best for his/her own selfish genes, I conclude that my own best course of action is to desert first. [... $]^{6}{ }^{\cdot 104}$

Das tierliche Individuum deutet in diesem Textausschnitt Verhaltensmöglichkeiten als Strategien im Sinne einer Investition, die den eigenen Kosten-Nutzen-Vorteil sichern soll. Die Wahl der richtigen Option ist jedoch abhängig vom Verhalten der/des Anderen und muss dahingehend wohlüberlegt sein - was zu der recht ausführlich von Dawkins ,durchgespielten“ Überlegung der geschilderten ,policy“ führt. Hier trifft sich das zugrunde liegende Modell der Finanzökonomie mit dem

\footnotetext{
${ }^{103}$ Vgl. Kirchgässner: Homo oeconomicus, S. 226.

${ }^{104}$ Dawkins: The Selfish Gene, S. 148 f. Hervorheb. im Original.
} 
theoretischen Handwerkzeug der Spieltheorie. Diese Partnerschaft ist fruchtbar: Boltanski und Chiapello sprechen von einer „Neuordnung des Kapitalismus“105 um die Finanzmärkte herum, die trotz bzw. gerade unter Einfluss der Kapitalismuskritik der 1960er Jahre den Markt als „Koordinationsmodus sozialen Handelns"106 bestärke. Dieser Markt ist jedoch nun ebenfalls neu geordnet. Vogl sieht durch u. a. die Entkopplung von Währung und Wertdeckung, die Revolution der Informationstechnologie und das damit beschleunigte Termingeschäft im letzten Drittel des 20. Jahrhunderts einen Markt institutionalisiert, der nicht mehr zur ökonomischen Theoriebildung führt, sondern die Finanztheorie nachahmt, bzw. ein Experimentierfeld darstellt, auf dem sich Wirtschaftstheorie verwirklichen kann. ${ }^{107}$ Es lasse sich daher von einem ,neue[n] Finanzkapitalismus“108 sprechen, in dessen Ordnung es ,weder Klassen noch Parteien, sondern nur noch das Band finanzieller Beteiligungen und ökonomischer Partner" ${ }^{\text {"109 }}$ gebe, und dessen soziales Feld in die temporale Dynamik des Finanzkapitals eingebettet sei. ${ }^{110}$ Wesentlich dafür sind spieltheoretische Ansätze. Die Spieltheorie wird in den 1940er und 1950er Jahren als formale Analyse von Konflikt und Kooperation im Bereich der Ökonomik entwickelt und breitet sich als Theorieansatz in den folgenden Jahrzehnten über die Verhaltensökonomik sowohl in die Evolutionsbiologie wie die Politikwissenschaft hinein aus. Als theoretisches Grundkonstrukt gilt hier: „In a strategic game, each player chooses a strategy (a rule specifying what action to take for each possible

${ }^{105}$ Boltanski, Luc/Chiapello, Ève: Der neue Geist des Kapitalismus. Konstanz 2003 (frz. 1999), S. 24.

${ }^{106}$ Boltanski/Chiapello: Der neue Geist, S. 48.

${ }^{107}$ Vgl. Vogl: Gespenst des Kapitals, S. 88 f., 107.

${ }^{108}$ Vogl: Gespenst des Kapitals, S. 111.

${ }^{109}$ Vogl: Gespenst des Kapitals, S. 112.

${ }^{110} \mathrm{Vogl}$ verfolgt das Entstehen von Finanzmärkten bis gegen Ende des 18. Jahrhunderts zurück. Voraussetzung sind die Kreditwirtschaft und der unendliche Aufschub der Krediteinlösung: Die Zirkulation in dieser Ökonomie ,,verläuft nicht über fortschreitende Kompensation [im geschlossenen Zyklus von Schuld und Tilgung], sondern über die endlose Proliferation einer uneinholbaren Schuld - eine Verzeitlichung, die sich im Vorhandensein des Nichtvorhandenen begründet und eine Unaufhörlichkeit im Systemverlauf programmiert" (Vogl: Gespenst des Kapitals, S. 81). Temporalität wird zu einer wesentlichen ökonomischen Determinante: Es entsteht so eine ,innere Zukunftssucht“ des Kapitalverkehrs, die Zukunft selbst wird produktiv gemacht. Den epochalen Umbruch der frühen 1970er Jahre sieht Vogl einerseits in der Aufkündigung der Währungsdeckung (mit dem Ende des Bretton-Woods-Abkommens von 1973) als „eine ökonomische condition postmoderne“ (S. 87, Hervorheb. im Original), in der sich „Währungen nur auf Währungen beziehen und direkt oder indirekt auf einem Standard ungedeckten Rechengelds beruhen“ (S. 87). Ganz Literaturwissenschaftler sieht Vogl darin eine „Auflösung von Wertreferenten“, „ein Regime flottierender Signifikanten ohne Anker und Maß, ohne die Sicherung durch ein transzendentales Signifikat“ (S. 87). Andererseits werden neue Finanzmärkte eingerichtet. Kennzeichnend für diese wirtschaftlich neue Epoche ist die Einrichtung neuer Finanzinstrumente (das Termingeschäft mit Devisen, Wertpapieren oder Hypotheken, die spekulative Risikoverlagerung und -investition, Finanzderivate etc.), die der ,Zukunftssucht ' die Vorstellung einer ,Aussicht auf die Beherrschung von Zeit, d. h. kontingenter Zukünfte“ (S. 95) beigesellt. 
information set) to maximize his or her expected payoff, taking into account that each of the other players is also making a rational strategic choice". 111

In Hrdys The Langurs of Abu unterscheidet der Sprachgebrauch deutlich die zugrunde liegenden Beobachtungen von den daraus schließenden Erläuterungen und verortet diese dabei in der neuen Episteme. Feldnotizenartige Schilderungen bilden einzelnes tierliches Verhalten ab: „At 10:38, Harelip charges into the midst of the Toad Rock troop. Splitear chases him out. Both males withdraw. Harelip is out of sight. Splitear sits in the crotch of an Erythrina tree grinding his teeth" (103, Hervorheb. im Original). Aber es sind die diese Beobachtungen bündelnden und erläuertenden Theoriepassagen, in denen sich eine durch die Terminologie des Finanzkapitalismus und spieltheoretischer Verhaltensmodelle geprägte ,neue' Evolutionstheorie ausdrückt. Zunächst geht es im Sinne einer klassischen Evolution um den wettbewerbsgesteuerten Kampf um Überleben und reproduktive Fitness: „Though it is not yet possible to conclusively show what langurs are defending when they fight, it is assumed here that conflict between groups reflects competition over prerequisites of survival and reproduction" (105, Hervorheb. MS). Dann werden gemeinsame Anteile, das ,stake holding“ wichtig: „But in general, members of the same troop share a stake in access to safe sleeping sites and adequate foraging areas, as well as the protection of immatures against marauding conspecifics" (105, Hervorheb. MS). Schließlich wird Verhalten spekulativ reflektiert:

The gain to an alpha male for fighting would usually be one of degree - that is, he might gain access to a temporary food source or access to more fertile females than are currently available in his own troop. The loss to him if injured, however, might be an absolute if he subsequently lost his harem due to injury-related debilitation. [...] [H] is future access to fertile females preemted. Furthermore, alpha males very rarely gain long-term benefits from taking over additional troops. (110)

Assuming that each invader who approaches a troop is attempting to maximize his access to sexually receptive females, the alternative strategies - haunting, attack-retreat, temporary joining, and take-over - have differential costs and quite different payoffs in terms of the overall reproductive success of the animals involved. Included in the final tally of success are not only the number of successfull inseminations but the physical costs and potential limitations on future possibilities uncurred by a policy of attack versus safer alternatives [...]. (128)

Diese Passagen sind intrikat mit dem spieltheoretischen Strategiebegriff verbunden. Verhaltensoptionen lassen sich hier gegeneinander aufrechnen, es geht nicht länger nur um den , survival of the fittest ' oder den , survival of the best adapted', sondern um eine Kosten-Nutzen-Rechnung, eine optimale Ressourcen-Aufwendung im Zeichen des idealen Investments. Der , final tally of success' lässt sich nicht mehr nur

\footnotetext{
${ }^{111}$ Dimand, Robert W.: Game Theory. In: Maryanne Cline Horowitz (Hg.): New Dictionary of the History of Ideas. Bd. 3. Detroit u. a 2005, S. 853.
} 
einfach - durch Befruchtungen - auszählen, sondern muss multifaktoriell berechnet werden, um die Zukunftsträchtigkeit einer Strategie prognostizieren zu können.

Das animal oeconomicum ist zudem nicht länger nur ein rationaler Akteur oder ein Stratege, es ist - auch dies wieder korrespondierend mit einem Wandel des homo-oeconomicus-Konzeptes - ein Unternehmer geworden. Verschiedentlich wurde in der Forschung und Kritik untersucht, wie die neue Form des Kapitalismus als ideologisches System neue Figuren und Modelle schafft. Marazzi spricht in diesem Zusammenhang von einer Metamorphose des homo oeconomicus, unter anderem in den Arbeiten des Sozioökonomen Gary Becker, von der ,idealtypische[n] Abstraktion der klassischen Ökonomie [...], ein[em] tauschende[n] Individuum, eine[m] der beiden Partner im Austauschprozess, deren Verhalten und Handlungsweisen, wie die Nutzentheorie ausführt, durch ihre Bedürfnisse bestimmt sind“, zu einem ,Unternehmer, und zwar ein[em] Unternehmer seiner selbst“. ${ }^{112}$ Das „Modell Investition-Kosten-Gewinn als Modell sozialer Beziehungen, als Modell der Existenz selbst“113 macht das Unternehmen ,zum universell verallgemeinerten gesellschaftlichen Modell“"114 im Neoliberalismus.

Im Forschungsverständnis der Ideengeschichte handelt es sich beim Neoliberalismus zwar um ein ,in sich heterogenes Phänomen“, ${ }^{115}$ das sich jedoch beschreiben lässt als:

an all-embracing economic and political ideology [...] that advocates the supremacy of the market over any alternative social arrangements, viewed from both a comparative and historical perspective, in ensuring the efficient allocation and utilization of scarce resources for the maximum satisfaction of relatively unlimited wants. The market, based on freedom of choice and respect for private property and individual rights, and underpinned by competition among producers and consumers alike, is seen as the ideal and optimal vehicle for the realization of human ends. Thus neoliberalism leads to the conclusion that individuals, rather than collectives, are the best basis for decision making and that the role of the state (or any similar collective agencies) should be limited to creating and ensuring an environment conducive to individuals freely and competitively making decisions and choosing between alternatives $\left[\ldots . .{ }^{116}\right.$

\footnotetext{
${ }^{112}$ Marazzi, Christian: Sozialismus des Kapitals. Zürich 2012, S. 16.

${ }^{113}$ Marazzi: Sozialismus des Kapitals, S. 14.

${ }^{114}$ Marazzi: Sozialismus des Kapitals, S. 15. Vgl. auch Boltanski/Chiapello: Der neue Geist, S. $91 \mathrm{ff}$.

${ }^{115}$ Biebricher, Thomas: Neoliberalismus zur Einführung. Hamburg 2012, S. 17. Biebricher weist zu Recht darauf hin, dass der Begriff des Neoliberalismus ein uneinheitliches Phänomen beschreibe, dass jedoch seine „begrifflichen Konkurrenten [etwa Marktwirtschaft, Kapitalismus, Postfordismus, fortgeschrittener Liberalismus] im Zweifelsfall noch größere konzeptionelle Probleme bergen“ und der Bedarf nach einem ,zeitdiagnostisch gehaltvollen Vokabular“, das die „sozialwissenschaftliche und polittheoretische Analyse der sozioökonomischen Gegenwart" trage, die akademische Verwendung des Einzelbegriffs rechtfertige (S. 16.).

${ }^{116}$ Mhone, Guy C. Z.: Neoliberalism. In: Maryanne Cline Horowitz (Hg.): New Dictionary of the History of Ideas. Bd. 4. Detroit u. a 2005, S. S. 1625-1628, hier S. 1625.
} 
Obwohl die zugrunde liegenden wirtschafts- und verhaltenstheoretischen Überlegungen wie beispielsweise der homo oeconomicus älter sind, fasst das ,neoliberale Paradigma“"117 erst mit den politischen Umwälzungen und weltweiten Krisen der 1970er Jahre auch in der Sphäre der praktischen Politik und, wie Kirchgässner in seinem Exkurs zur Soziobiologie beiläufig zeigt, in der akademischen Welt jenseits ökonomischer Theorie Fuß. ${ }^{118}$ Ausgangspunkt für diesen Siegeszug des Neoliberalismus als , alternativloser Ideologie“ ist für die Forschung die Rezession von 1973, die zu einer radikalen Neukonzeption politischer und wirtschaftlicher Strategien führt. Ergebnis ist laut Wolfgang Maderthaner, dass der Markt noch deutlicher als zuvor ,zur eigentlichen Instanz, zum essenziellen Indikator allen menschlichen Handelns und Tuns“, zu ,jener spontanen Ordnung, in der sich das millionenfach individuell verstreute Wissen koordiniere und verdichte" ${ }^{119}$ wird. Neoliberalismus lässt sich so trotz der darin versammelten unterschiedlichen wirtschaftstheoretischen und -praktischen Ansätze als Ideologie begreifen, weil er mit Mario Candeias als ,Form der Rationalisierung“ das „organisierende Element einer krisenhaften Transformation aller gesellschaftlichen Verhältnisse [...] im Alltagsverstand einnistet, damit Teil der ,gegenständlichen Welt “ wird“". ${ }^{120}$ Diesen Glauben an den Markt als allem menschlichen Handeln zugrunde liegendes Modell und als Ordnungsprinzip nennt Joseph Vogl in seiner Untersuchung des Gespenst des Kapitals die Oikodizee, ,die Überzeugung nämlich, dass das Marktgeschehen ein exemplarischer Schauplatz von Ordnung, Integrationsmechanismen, Ausgleich, sinnvollen Allokationen und somit von gesellschaftlicher Vernunft sei und insgesamt an eine kohärente, systematische Darstellungsform appelliere". ${ }^{121}$ Diese Oikodizee beinhaltet erstens die Überzeugung, dass ,der

\footnotetext{
${ }^{117}$ Maderthaner, Wolfgang: Die Ökonomie des Okkulten - Anmerkungen zu einer Geschichte des Neoliberalismus. In: Andrea Grisold/Wolfgang Maderthaner/Otto Penz (Hg.): Neoliberalismus und die Krise des Sozialen. Wien 2010, S. 13-62, hier S. 28.

${ }^{118}$ Vgl. Maderthaner: Die Ökonomie des Okkulten, S. 29 f.; Kirchgässner: Homo oeconomicus, S. $215 \mathrm{ff}$.

${ }^{119}$ Maderthaner: Die Ökonomie des Okkulten, S. 35 f.

${ }^{120}$ Candeias, Mario: Konjunkturen des Neoliberalismus. In: Christina Kaindl (Hg.): Subjekte im Neoliberalismus. Marburg 2007, S. 9-17, hier S. 10. Den Ideologiebegriff sehen auch Boltanski/ Chiapello anwendbar. Denn, so die Autor/innen, Ideologien garantieren die Reproduktion der jeweiligen Gesellschaftsordnung; ihre Wirkung besteht darin, dass „die Menschen durch sie ihr Lebensumfeld nicht als unerträglich wahrnehmen.“ Der Neoliberalismus stütze sich ,auf eine Reihe von handlungsanleitenden Vorstellungen und gängigen Rechtfertigungsmodellen [...], durch die er als eine annehmbare und sogar wünschenswerte, allein mögliche bzw. als beste aller möglichen Ordnungen“ erscheint. Als „Gesamtheit von Glaubensätzen, die mit der kapitalistischen Ordnung verbunden sind und zur Rechtfertigung dieser Ordnung, zur Legitimation und mithin zur Förderung der damit zusammenhängenden Handlungsweisen und Dispositionen beitragen", sehen Boltanski und Chiapello mit Bezug auf Max Webers Verwendung in diesem neuen ,Geist des Kapitalismus‘ eine dominante Ideologie. Boltanski/Chiapello: Der neue Geist, S. 46. $\mathrm{Zu}$ den theoretischen Grundlagen des Neoliberalismus vgl. Biebricher: Neoliberalismus, S. $24 \mathrm{ff}$.

${ }^{121}$ Vogl: Gespenst des Kapitals, S. 28.
} 
Markt‘ solchermaßen als explikatorisches Grundmodell für alles herangezogen werden kann, zweitens aber auch das Vertrauen, dass ,der Markt' sinnvoll, ausgeglichen und vernunftsgemäß, handelt", sofern er nicht von äußeren (meist staatlich gedachten) Einflüssen manipuliert werde. Die Problematik dieses illusorischen Denkens hat Vogl bereits ausgeführt. Sie lässt sich jedoch um die bereits diskutierte dritte Ebene einer Ökonomisierung der Biologie bzw. Biologisierung der Ökonomie, wie sie deutlich in der zeitgenössischen primatologischen Forschung vollzogen wird, erweitern. Das von Vogl in seinem Oikodizee-Begriff formulierte ideologische Grundvertrauen führt dazu, dass sich Evolutionstheorie und Ökonomie/Ökonomik nicht nur gegenseitig befruchten, sondern in einer Art ideologischem Zirkel auch gegenseitig bestätigen. Hrdys Languren leben in sozialen Formierungen, deren Zyklizität dem des Marktgeschehens gleicht. Sie sind Akteure, Strategen, Investoren und bestätigen wiederum dadurch, dass sie in der ökonomisierten Sprache der Soziobiologie gleichsam Grundoperationen primatischen Verhaltens abzubilden scheinen, das neoliberale Paradigma des Unternehmers als universellem Grundmodell evolutionär.

Dass die theoretischen Voraussetzungen für die Ausbildung einer neuen Form kapitalistischen Denkens unter dem Schlagwort ,Neoliberalismus ' sich im Sprachgebrauch eines ethologischen Forschungsberichtes von 1977 ablagern, halte ich eher für eine Konfluenz als für eine Koinzidenz. Sie bestätigt die wissenschaftstheoretische Grundthese, dass auch Naturwissenschaften als gesellschaftliche Praxen Bestandteil von Kultur(en) sind und daher nicht nur in ihren Geltungsansprüchen kulturimmanenten Rationalitätsnormen unterworfen, sondern auch unauflöslich mit anderen Praxisformen verbunden bleiben. ${ }^{122}$ In diesem Fall sind die Ethologie und der Beginn dieses neuen Paradigmas in den 1970er Jahren unweigerlich miteinander verwoben.

\subsubsection{Der Markt feministischer Interessen}

Was hat nun ein ökonomisiertes Tiermodell und die Partnerschaft von Biologie und Ökonomie in der Geburtsstunde des neoliberalen Paradigmas mit Emotionalität in der Forschung zu tun? Das oben angeführte Rationalitätsproblem des homo oeconomicus/animal oeconomicum betrifft schließlich eher den Untersuchungsgegenstand der Primatologie als ihre Forschenden. Zunächst ist für die Beantwortung dieser Frage wesentlich, warum eine feministisch interessierte Primatologie Ende der 1960er und Anfang der 1970er Jahre so bereitwillig die neuen ökonomisch inspirierten Theorien und Modelle der Soziobiologie übernimmt. ${ }^{123}$

\footnotetext{
${ }^{122} \mathrm{Vgl}$. Lettow, Susanne: Biophilosophien. Wissenschaft, Technologie und Geschlecht im philosophischen Diskurs der Gegenwart. Frankfurt a. M. u. a. 2011, S. 16 f.

${ }^{123}$ Dass sich ein politisch und epistemologisch feministischer Diskurs in der Primatologie bemerkbar macht, ist bereits verschiedentlich reflektiert worden. Besonders Donna Haraway hat sich in Primate Visions damit beschäftigt, wie Primatologinnen vor allem der zweiten Generation an der „dense intersection of western feminism, multicultural and global feminism, decolonization, and multinational capitalism" arbeiten: wie sie ihren Fokus auf weibliche Tiere und deren Rolle und Funktion für primatisches Sozialleben legen, neues Wissen darüber zutage fördern, was ,being
} 
Die zeitgenössische primatologische Forschung wird immer weiblicher: ${ }^{124}$ Sowohl in der Praxis als auch in der Theorie stehen ab den späten 1960er Jahren zunehmend Weibchen im Vordergrund der Studien, welche ihrerseits immer häufiger von weiblichen Forschenden ausgeführt werden. Dabei arbeiten diese Primatologinnen häufig unter der pragmatischen Prämisse, dass eine empathische und emphatische Identifikation mit dem ,eigenen“ Geschlecht in der Primatologie zu Ergebnissen führt: Der zuvor recht androzentrische Fokus der Primatologie soll ausgeglichen werden durch die Forschung von Frauen an Weibchen. Kritische Arbeiten dekonstruieren unter diesem Einfluss z. B. die traditionelle Theoriebildung der Primatologie, entwerfen alternative theoretische Modelle oder erweitern bestehende hinsichtlich einer geschlechterübergreifenden Universalität. ${ }^{125}$ Die individuelle Praxis empathischer Beobachtung im Feld weicht dabei jedoch zunehmend einer Instrumentalisierung von Empathie als ,part of the western scientific tool kit [...] in constant productive tension with its twin, objectivity“. ${ }^{126}$ Die zeitgleich sich institutionalisierende Soziobiologie bietet sich als Instrument an, um der u. a. durch die Arbeiten der ,Trimates ' unterfütterten Konnotation von Weiblichkeit und Empathie zu entkommen:127 Durch zweckrationalistische Argumentation in einem marktorientierten Modell lässt sich das Problem der ,gegenderten“ Empathie umgehen. Denn dieses Erklärungsmodell benötigt keine empathischen Perspektivübernahmen mehr, da alle Akteure und Akteurinnen im Spektakel des Feldes streng theoriekonform im Sinne eines Eigeninteresses handeln, das seinerseits durch die Verlagerung auf die Ebene der Gene emotional bereinigt wurde. Gene sind ,ruthlessly selfish', wie Dawkins schreibt, sie sind aber keine emotionalen Akteure. Die von ihnen programmierten Fleischmaschinen kennen folglich auch keine anderen Emotionen als ein zweckrationales

female “ für Primaten im ,age of bio-politics“ bedeutet, und welche Argumente sie in den zeitgenössischen Geschlechterdiskurs einfließen lassen. Haraway: Primate Visions, S. $288 \mathrm{ff}$. Nicht nur nimmt Hrdy mit ihren Forschungsarbeiten, deren Fokus auf den evolutionären Verhaltensstrategien von Weibchen liegt, deutlich an diesem neuen Programm der Primatologie teil. Mit ihrer nächsten Publikation von 1981 entwirft sie sogar eine feministische Evolutionstheorie, vgl. Hrdy, Sarah Blaffer: The Woman That Never Evolved. Cambridge 1981.

${ }^{124}$ Vgl. Haraway: Primate Visions, S. $293 \mathrm{ff}$.

${ }^{125}$ Vgl. z. B Fedigan: Primate Paradigms; Rowell, Thelma: The Concept of Dominance. In: Behavioral Biology 11 (1974), S. 131-154.

${ }^{126}$ Haraway: Primate Visions, S. 293. Haraway verweist zugleich auf den Frau/Natur/Emotions-Nexus, der weibliche Forschende zwingt, sich im Dienste wissenschaftlicher Glaubwürdigkeit stärker als ihre männlichen Kollegen vom Anthropomorphismus- und Emotionalitätsvorwurf zu distanzieren (S. 249 f.), und auf die Tendenzen zu , universalizing, identification, and denial of difference" (S. 293), die in einer empathischen Aneignung des ,Anderen“ in der Primatologie und Ethnologie liegen.

${ }^{127} \mathrm{Zu}$ einem Überblick über eine solche „variet[y] of feminist work on emotion“, die Emotion als „one of the centers of a revalorized feminity“ ansetzt und damit den Dualismus von Emotion und Rationalität sowie „the association of women with nature“ in den 1970ern verstärkt, siehe Lutz: Feminist Theories, S. 348. 
Interesse ${ }^{128}$ - an Überleben und Fortpflanzung. An den Tieren selbst wird daher keine Emotionalität mehr thematisiert. Hrdy schreibt zwar in den Beobachtungsnotizen, dass ein Männchen nach einer Auseinandersetzung mit den Zähnen knirscht. Was in einem anderen Forschungsparadigma als affektiver Ausdruck gewertet werden konnte, spielt aber in ihrer Interpretation des Verhaltens keine Rolle mehr. Das Interesse des animal oeconomicum - z. B. Zugang zu Weibchen, Zugang zu Futter - ist mit Vogls Interesse-Verständnis zwar nicht leidenschaftslos, wird aber zumindest nicht als affektives diskutiert. Dort, wo sich Anlass böte, über Affekte zu schreiben, wird ökonomisiert: So ist „,maternal behavior“ - ein traditionell affektiv besetztes Thema - ,relatively unsurprising, since by caring for her offspring, the mother invests in her own genetic representation in subsequent generations" (198). Damit entlastet Hrdy das weibliche Tier (und in der typischen soziobiologischen Verlängerung weibliche Primaten generell) von einem als affektiv und irrational konnotierten , mütterlichen Instinkt ${ }^{\star}$ und macht es zur rational handelnden Akteurin in einem Investmentschema.

Donna Haraway, die verschiedene primatologische Wissenschaftlerinnen dieser epistemischen Wendezeit untersucht - neben Hrdy auch Jeanne Altmann, Linda Marie Fedigan und Adrienne Zihlmann -, sieht daher in Sarah Blaffer Hrdys Arbeiten noch einen anderen Vorteil der Soziobiologie für eine kritische feministische Epistemologie: ${ }^{129}$ Durch die Grundeinheit des Individuums werden die Langurenweibchen wie die Männchen nicht nur in die „logics of investment and expansion in late capitalism“130 eingebettet. Als ,agents, citizens, and subjects in the great dramas of evolution" 131 werden sie auch voneinander unterschieden. Eher als ein Modell des Primatenweibchens oder -männchens und damit eine Biologisierung von Geschlechterrollen kreiert Hrdy damit Heterogenitität, Differenz und Individualität als Modell für Primat/innen.

Haraway hebt in ihrer Analyse von Hrdys Forschungsprogramm das Dramatische ihres vermeintlich emotionalen Forschungsgegenstandes - Infantizid - hervor. Damit nimmt sie m. E. Hrdys Aussage, es sei das „high drama“ (76) des Langurenlebens, das sie jeden Tag zur Arbeit bewogen habe, zu sehr als affektives Indiz wahr. Es lässt sich argumentieren, dass das Abrufen der dramatischen

\footnotetext{
${ }^{128}$ Zur Wort- und Begriffsgeschichte des Interesses bzw. seinem Wandel von der primär juristischen über die ökonomische zur affektiven und schließlich ästhetischen Bedeutung siehe auch Wölfel, Kurt: Interesse/interessant. In: Karlheinz Barck u. a. (Hg.): Ästhetische Grundbegriffe: Historisches Wörterbuch in sieben Bänden. Bd. 3. Stuttgart/Weimar 2001, S. 138-174.

${ }^{129}$ Haraway ist daran interessiert, wie die Wissenschaftlerinnen ,engage in a complicated dance in their textual and professional politics around constructing isomorphisms or non-congruences among the potent entities that emerge as actors in their accounts called female, woman, and scientist". Während Altmann, Fedigan und Zihlmann sich im Laufe der 1970er Jahre zunehmend kritisch mit den soziobiologischen Ansätzen auseinandersetzen, die hier diskutiert wurden, ist Hrdy laut Haraway als ,unrepentant sociobiologist“ zu betrachten. Vgl. Haraway: Primate Visions, S. 350.

${ }^{130}$ Haraway: Primate Visions, S. 352.

${ }^{131}$ Haraway: Primate Visions, S. 350.
} 
Qualität primatischen Sozialverhaltens bereits eine topische Tradition der mehr oder minder subtil mit einem Theatermodell der Beobachtung liebäugelnden Feldprimatologie geworden ist. ${ }^{132}$ Dagegen scheint Hrdys neues theoretisches Modell gerade die Neutralisierung jeglichen potenziell emotionalen ,Dramas ' zu begünstigen. Die Soziobiologie, die Hrdy mit ihrem The Langurs of Abu vertritt, kennt kaum Emotionen - nicht bei den als rationale, individuelle Strateg/innen konzipierten Tieren, deren Motivation immer eine der kalkulierten, individuellen Nutzengewinnung ist. Aber auch dort, wo die Forscherin selbst in ihrem Text als „Ich“ auftritt, herrscht emotionale Zurücknahme. Diese Auftritte dienen der Darstellung der Forschungssituation (,I returned in the dry season months of February and March, 1973“, 251; „During the 1975 study period, I had the opportunity to test explicit predictions based on the hypothesis that $\left.[\ldots]^{\prime \prime}, 173\right)$, nicht der emotionalen Auskunft. Überwiegend verbleibt der Bericht in einem szientistisch unpersönlichen Erzählmodus: ,When considering her antagonism towards Splitear during estrus, one should keep in mind [...]" (171). Hrdy beschränkt sich trotz oder gerade wegen des herausfordernden Themas des Infantizids auf sehr wenige Passagen, in denen es um emotionales Erleben geht. So heißt es an einer Stelle, in der sie evaluiert, ob es sich bei dem beobachteten Phänomen wie vermutet um Infantizid handle:

\begin{abstract}
Although infanticide was foremost on my mind when I decided to study langurs, its actual occurence seemed totally implausible. Despite Shifty's replacement of Mug, despite the fact that all six infants were missing, despite reports by two local people who had seen an adult male langur kill infants in the hillside troop's home range, I grasped at straws. I spent a whole day trying to convince myself that this was a different troop, one without infants $[\ldots]$. But the longer I peered through the mist at those rain-soaked, skittish females, the more I realized they were unmistakably, Bilgay, Itch, Harrieta, Oedipa, Pawless and Sol. (243)
\end{abstract}

An anderer Stelle geht es um das ,unmütterliche' Verhalten, das Langurenmütter zeigen. Hier berichtet Hrdy zwar von dem Effekt, den ,this nonchalance on the part of the mothers and the heart-rendering screams of their infants" auf sie als „,nullipara on my own right“ (237) habe: ,[It] made it stressful for me to concentrate on infants for hours on end. It was a relief that in January 1974 I laid

\footnotetext{
${ }^{132}$ So schreibt etwa Barbara Smuts: „Many primate field workers compare their jobs to watching soap operas, except that the characters are real and they do not speak. This is an apt comparison because what captured my interest and motivated me to return to observe my subjects again and again was the daily drama of baboon life" (SF, S. 8, Hervorheb. MS). Goodall sucht sich zum Ausleben ethologischer Schaulust (vgl. Abschn. 3.2.1) ,,a place where I could stand and watch“ und nimmt das Beobachtete als „scene“ wahr (TW, S. 8); die Boeschs sprechen vom „dramatic event“. Boesch/Boesch-Achermann: The Chimpanzees of the Taï Forest, S. 34. Die Leiterin der kongolesischen Bonobo-Schutzstation Lola ya Bonobo, Claudine André, wählt grundsätzlich Metaphern des Dramatischen für ihre Memoiren: „Chaque tragédie que traverse ce pays est une petite mort. [...] Mon drame de vie n'est que broutille face à la tragédie humaine“. André, Claudine: Une tendresse sauvage. Paris 2006, S. 100. Hervorheb, MS.
} 
aside the problem of allomothers to move on to other topics. I felt that I had found answers to many of the questions originally posed" (237). Als Hrdy von einem Weibchen in Östrus angegriffen wird, ,I was so startled by a sudden charge [...] that I stepped backwards into a crevice" (171 f.).

Diese wenigen Passagen verweisen zwar auf eine Art affektiver Reaktion. Sie bleibt aber unter etablierten Formulierungen einer Forschungsemotionalität angedeutet, deren Fokus nicht beim forschenden Individuum, sondern beim Gegenstand und bei der Ökonomie des Forschens liegt: Infantizid wirkt ,totally implausible " und wird daher als logischer Schluss erst nach einiger Zeit zugelassen. Die Überraschung führt andeutungsweise zu einem Unfall - Hrdy fällt in eine Felsspalte -, hat aber keine weiteren affektiven (oder narrativen) Folgen. Emotionaler Stress bei der Beobachtung und die Erleichterung über ein abgeschlossenes Projekt stehen nicht im Zusammenhang mit einer Form intersubjektiver emotionaler Beziehung wie dies bei den ,Trimates' der Fall war. Obwohl Hrdy ihre Forschungstiere mit Eigennamen benennt und als „Cast of Characters" (76) in ihrem Bericht führt, steht sie diesen Tieren laut ihrem Text nicht näher als den Charakteren einer „,colobine soap opera“, die ihr Interesse weckt. Wenn Hrdy emotionale Zustände erwähnt, geht es nicht um empathische Perspektivierungen, die Verständnis produzieren würden. In dieser Darstellung wird das Affektive nicht als epistemologisches Instrument oder als Begleiterscheinung der Forschungsarbeit in Erwägung gezogen, sondern nur im Zusammenhang der Ausführbarkeit der Verhaltensbeobachtung und der Aufrechterhaltung des Forschungsinteressen betrachtet. ${ }^{133}$ Dieses Interesse ist als ,interest“ doppeldeutig, es ist Interesse und Investment zugleich: ${ }^{134}$ So lässt sich auch das vermeintliche ,high drama“ deuten, das Hrdy für die Languren angibt: Weniger im Sinne einer Affektdramaturgie, wie sie das Drama als theatrale Gattung vorgibt, denn als unterhaltender Anreiz für das Zeit- und Arbeitsinvestment einer Primatologin, die sich als „no true naturalist“ (76) beschreibt:

[I]f it were not for the fact that langurs interact as individuals, I could never have sustained my interest in them for five years. It was the high drama of their lives, $[\ldots]$ that got me out of bed in the morning and kept me out under the Indian sun, tramping about their haunts for eleven hours at a stretch. (76)

Dass Hrdy die primatologische Beobachtungssituation in ihrer theatralen Qualität nicht in Hinsicht auf eine Affektdramaturgie deutet, wird schon in ihrer Präambel zu The Langurs of Abu deutlich. Das ,high drama' des Langurenlebens wird von Hrdy dort mit William Shakespeares unpopulärem Drama Titus Andronicus verglichen:

\footnotetext{
${ }^{133}$ Das Affektive hat hier in etwa den Stellenwert ,klassischer ' Erkenntnisaffekte, wie Daston/ Park ihn für das Staunen in der Frühen Neuzeit untersuchen. Siehe Daston/Park: Wonders.

${ }^{134}$ Der Begriff ,interest' ist etymologisch älter in der Bedeutung ,the possession of a share in or a right to something" als als kognitive Anteilnahme, vgl. interest, n. In: OED Online (2018), https://www.oed.com/view/Entry/97735 (21.03.2018). Siehe auch Wölfel: Interesse/interessant.
} 
Small wonder that a play replete with infanticide, dismemberment, rape, and cannibalism has not weathered well the test of time. To modern audiences, these events seem implausible and unnatural. Nevertheless, if more primatologists had seen this play before going off into the field, they might better have understood the behavior unfolding before them in the savannas and forests where monkeys are studied. (1)

Vorgeblich wird hier Shakespeares Theatertext den Leser/innen (und den wissenschaftlichen Kolleg/innen) als kultureller Zugang für das ,Drama' des primatischen Soziallebens empfohlen. Doch dann heißt es weiter:

The conflict of interest between Aaron and his mistress is basic to all sexually reproducing creatures where the genotypes - and hence self-interests - of two consorts are necessarily not identical. The survival of the empress and her previous children [...] depends upon her continued marriage to her powerful husband. The illegitimate infant, however, may be Aaron's sole opportunity to sire a son. It is to the advantage, then, of one consort, but not the other, for this child to live. (1 f.)

Was Hrdy hier betreibt, ist keine ,Kulturalisierung der Natur, sondern ganz im Gegenteil eine Biologisierung des Shakespeare-Plots, sodass dieser nur mehr einer universellen Verhaltenskonstante unter dem Schirm einer speziellen Verhaltensökonomie zu gleichen scheint. Das ,high drama“ der starken Emotionalität, das in diesem Shakespeare-Drama aufgeführt wird, ist bei Hrdy schlicht ein evolutionäres Programm, das von den Akteur/innen eines biologischen Grundplots ausgeführt wird.

\subsubsection{Barbara Smuts' Sex and Friendship in Baboons (1985)}

\subsubsection{Die Rückkehr der Emotion als Ressource}

Diese Entfernung der Emotionalität aus der Forschung als Begleiterscheinung eines starken theoretischen Modells ist $\mathrm{zu}$ verorten in jener ersten Phase, in der sich das neoliberale Paradigma etabliert. Mit Luc Boltanski und Ève Chiapello lassen sich unter der These, dass es gerade die verschiedenen Protestkulturen der 1960er und 1970er Jahre und ihre ausgeprägte Kapitalismuskritik sind, die zu einem Wandel der Funktionsweisen und Strukturen des Kapitalismus führen und ihn gestärkt daraus hervortreten lassen, zwei Perioden identifizieren, in denen sich dieser ,neue Geist des Kapitalismus' entfaltet: Die erste, in der Hrdys Langurs zeitlich angesiedelt ist, betrifft die Jahre 1968-1978, die zweite, in der ich Smuts Sex and Friendship verorte, die Jahre 1985-1995. ${ }^{135}$ Diese Einteilung in verschiedene Phasen oder ,Konjunkturen“ findet sich auch anderweitig. Dabei zeichnet sich z. B. bei Candeias die erste Phase durch ,einen molekularen Prozess der Zersetzung des alten geschichtlichen Blocks und der Durchsetzung neoliberaler Reformen“136 aus, der auf „eine Restauration/Revolution des gesamten Ensembles

\footnotetext{
${ }^{135}$ Vgl. Boltanski/Chiapello: Der neue Geist, S. 213 ff.

${ }^{136}$ Candeias: Konjunkturen des Neoliberalismus, S. 11.
} 
der gesellschaftlichen Verhältnisse, einschließlich der Subjektivitäten“, 137 abziele. Dies betrifft vor allem auch den Stellenwert von Emotionalität und den Umgang mit Gefühlen.

Verschiedentlich wurde in der jüngeren Forschungsliteratur bereits darauf hingewiesen, wie Emotionalität im Zeichen des Neoliberalismus als Ressource reorganisiert und kultiviert wird und welche entscheidende Rolle sie im semantischen Feld des ,Humankapitals' spielt. Eva Illouz untersucht in ihrer gleichnamigen Monographie Gefühle in Zeiten des Kapitalismus unter der These, dass „die Bildung des Kapitalismus Hand in Hand ging mit der Bildung einer stark spezialisierten emotionalen Kultur". ${ }^{138}$ Illouz' Argumentation setzt eher auf Kontinuität als auf Fluktuation von Emotionalität im Kapitalismus. Sie sieht eine Kooperation von sich zeitgleich mit der ,klassischen Ökonomie“ profilierenden Ansätzen wie der Psychoanalyse und Management-Theorien, die dazu führt, dass Emotionalität aus der Sphäre des Privaten herausgeschält und später unter den neuen Leitaufgaben der Kommunikation und Kooperation im Unternehmensmodell zu einem nötigen Element der ökonomischen Sphäre gemacht wird. ${ }^{139}$ Ergebnis ist ein emotionaler Kapitalismus als ,eine Kultur, in der sich emotionale und ökonomische Diskurse und Praktiken gegenseitig formen“. ${ }^{140}$ Affekte werden darin ,zu einem wesentlichen Bestandteil ökonomischen Verhaltens", das emotionale Leben aber wird zugleich „,der Logik ökonomischer Beziehungen und Austauschprozesse“"141 unterworfen. Der ,emotionale Kapitalismus“ ordnet seine Welt neu, ,indem er das ökonomische Selbst emotionaler und die Emotionen instrumenteller machte". ${ }^{142}$

\footnotetext{
${ }^{137}$ Candeias: Konjunkturen des Neoliberalismus, S. 11.

${ }^{138}$ Illouz, Eva: Gefühle in Zeiten des Kapitalismus. Frankfurt a. M. 2006, S. 12.

${ }^{139}$ Auch Illouz dient hier die Analyse der emotionalen Dimension des Kapitalismus zur Kritik an der bereits diskutierten Vorstellung einer fortschreitenden Entemotionalisierung und Rationalisierung als Charakteristikum der Moderne. Dem setzt sie ihre Geschichte des ,Aufstiegs des Homo Sentimentalis' entgegen und ergänzt eine solche Kritik durch eine Analyse, die aufzeigt, wie wenig stabil vergeschlechtlichende Konstruktionen von Emotionalität sozialgeschichtlich sind. Vgl. Illouz: Gefühle, S. 12 f. Landweer/Newmark sehen ähnlich eine Vereinbarkeit des Aufsuchens ,echter' Gefühle in Psychotherapie und Erlebniskultur mit der Zweckrationalisierung des emotionalen Managements in den neuen Arbeitskulturen. Dies seien lediglich die beiden durch die systematische, aber unrealistische Trennung Freizeit/Arbeit existierenden Seiten der Emotionskultur unserer Gegenwart. Vgl. Landweer/Newmark: Zur Rhetorik von Emotionsdebatten, S. $101 \mathrm{ff}$.

${ }^{140}$ Illouz: Gefühle, S. 13.

${ }^{141}$ Illouz: Gefühle, S. 13.

${ }^{142}$ Illouz: Gefühle, S. 41. Wie Haraway sieht auch Illouz die zweite Welle des Feminismus als Komplizin in diesem Prozess. In der Aufwertung von Sorge/Pflege und Autonomie/Selbständigkeit habe sie das therapeutische Narrativ der Psychologie, das die Emotionalität erst in eine diskursive Sphäre gezerrt habe, in der sie als Ressource erkennbar werden konnte, vollständig begrüßt und gefördert. Denn die „Kontrolle der Emotionen, die Klärung der eigenen Werte und Ziele, der Gebrauch der Kalkulation und die Dekontextualisierung und Objektivierung der Emotionen - all das impliziert eine Intellektualisierung intimer Bande im Namen eines größeren moralischen Projektes: der Herstellung von Gleichheit und fairen Bedingungen des Austausches durch rückhaltlose verbale Kommunikation über die eigenen Bedürfnisse, Emotionen und Ziele“ (S. 56, Hervorheb. im Original).
} 
Gertraud Koch greift Illouz' Thesen eines derart emotionalen Kapitalismus und der gegenseitigen Durchdringung von Emotionalem und Ökonomischem auf und spricht von „emotive technologies“"143 wie z. B. Empathiefähigkeit und Emotionsmanagement, die einer ,Verwertungslogik des Gefühls“144 als Teil des emotionalen Regimes des Neoliberalismus dienen. Birgit Sauer spricht gar in Hinblick auf die neue ,mediale, politische und wissenschaftliche Aufmerksamkeit für Emotionen" 145 im Rahmen des ,Affective Turn“ von einer , affektiven Gourvernementalität‘ als Teil neoliberaler Biopolitik. Der neue Gefühlsdiskurs ist Sauer „Ausdruck und zugleich Movens neuer Formen von Selbstverständnissen und Identitätsentwürfen im Neoliberalismus, neuer politischer Subjektivierungsweisen sowie veränderter Vorstellungen von citizenship, von politischen und sozialen Rechten“. ${ }^{146}$ Auch Christina Kaindl, die sich ebenfalls mit dem foucaultschen Gouvernementalitätsbegriff beschäftigt, konstatiert ein neues Emotionalitäts- und Motivationsdispositiv. Anders als Illouz geht Kaindl von einer Diskontinuität aus und grenzt die Kapitalismusformen Fordismus und Neoliberalismus hinsichtlich ihres Umgangs mit Emotionalität voneinander ab. Im Fordismus werden.

\begin{abstract}
Gefühle im Rahmen restriktiver Handlungsfähigkeit [...] durch Polarisierung ruhig gestellt [...] [,] einerseits in der scheinhaften ,Verinnerlichung ' der Emotionalität als von den realen Lebensbedingungen isolierter, bloß , subjektiver' Zustand des je einzelnen Individuums, andererseits als ,Entemotionalisierung ‘, d. h. Zurückgenommenheit und Unengagiertheit des Handelns [...]. ${ }^{147}$
\end{abstract}

Im neoliberalen Mobilisierungsdiskurs dagegen werden Gefühle profanisiert und jederzeit einsetzbar:

Sie werden (auch hier) nicht als Bewertung der Situation gedacht, sondern müssen unabhängig davon zum Handeln unter fremd gesetzten Zielen befähigen, sind Teil von Selbstinstrumentalisierungen, die die geforderten Haltungen - aktiv, kreativ, demütig bereitstellen können. ${ }^{148}$

\footnotetext{
${ }^{143}$ Koch: ,Feeling Rules', S. 60.

${ }^{144}$ Koch: ,Feeling Rules', S. 61.

${ }^{145}$ Sauer, Birgit: Affektive Gouvernementalität. Eine geschlechtertheoretische Perspektive. In: Elisabeth Mixa u. a. (Hg.): Un-Wohl-Gefühle. Eine Kulturanalyse gegenwärtiger Befindlichkeiten. Bielefeld 2016, S. 147-162, hier S. 150.

${ }^{146}$ Sauer: Affektive Gouvernementalität, S. 150, Hervorheb. im Original.

${ }^{147}$ Kaindl, Christina: Frei sein, dabei sein: Subjekte im High-Tech-Kapitalismus. In: Christina Kaindl (Hg.): Subjekte im Neoliberalismus. Marburg 2007, S. 141-161, hier S. 156, Hervorheb. im Original.

${ }^{148}$ Kaindl: Frei sein, dabei sein, S. 157. Folge dieser Ökonomisierung der Gefühlskultur in einem emotionalen Kapitalismus für das Subjekt kann u. a das Phänomen eines ,erschöpften Selbst ${ }^{*}$ sein, vgl. Ehrenberg, Alain: Das erschöpfte Selbst. Depression und Gesellschaft in der Gegenwart. Frankfurt a. M. 2008 (frz. 1998).
} 
Wie Kaindl den Manager und Arbeitsmarktreformer Peter Hartz zitiert: Emotion wird zu Kapital. ${ }^{149}$

Ich möchte diese Thesen hier aufgreifen und Smuts' Text daraufhin untersuchen, welchen (neuen) Stellenwert Emotionalität darin einnimmt. Verweist Hrdys The Langurs of Abu darauf, dass soziobiologische Theorie noch Teil hat an der in Kaindls Sinn fordistischen Entemotionalisierung, insofern sie gerade eine solche Zurücknahme und Unengagiertheit des Verhaltens/Verhandelns propagiert, so lässt sich eine Rückkehr der Emotionen als Ressource im Zeichen eines emotionalen Kapitalismus an Barabara Smuts' keine zehn Jahre später erscheinenden Studie Sex and Friendship in Baboons beobachten.

\subsubsection{Emotionale Strategien unter Pavianen}

Barbara Smuts' Sex and Friendship in Baboons beginnt mit einem Vorwort des Anthropologen und Evolutionsbiologen Irven DeVore. DeVore war Schüler Sherwood Washburns und Lehrer Robert Trivers. Wie Sarah Blaffer Hrdy, die ebenfalls bei DeVore studierte, lässt sich auch Barbara Smuts durch diese akademische Netzwerkgenealogie als Soziobiologin einordnen. Zugleich stammt DeVore aus Jane Goodalls Alterskohorte und begann seine Pavian- zeitgleich mit Goodalls Schimpansenstudien in Gombe. So überrascht es nicht, dass es in seinem Vorwort heißt: „Those who have been privileged to watch baboons long enough to know them as individuals and who have learned to interpret some of their more subtle interactions will attest that the rapid flow of baboon behavior can at times be overwhelming“. ${ }^{150}$ Das Kennenlernen der Tiere als Individuen, die Interpretation ihrer subtilen Interaktionen und die Überwältigung in und von der Forschungssituation sind bekannte Themen der Episteme der ,Trimates'. Barbara Smuts' Studie wird in DeVores Vorwort jedoch als Vorbote der Primatologie der 1980er Jahre angekündigt, denn

adhering to the most scrupulous methodological strictures, the author yet maintains an open research strategy - respecting her subjects by approaching them with an open mind of an ethnographer and immersing herself in the complexities of baboon social life before formulating her research design, allowing her to detect and document a new level of subtlety in their behavior. ${ }^{151}$

\footnotetext{
${ }^{149}$ Vgl. Kaindl: Frei sein, dabei sein, S. 157. Das vollständige Zitat lautet: „Wir springen auf eine dritte Achse im Raum der Möglichkeiten: Emotionalität. Was fühlen wir? Hinter dem Begriff Qualität steckt die Suche nach den Grenzen der Empfindung. Emotion wird zu Kapital“. Hartz, Peter: Job-Revolution. Wie wir neue Arbeitsplätze gewinnen können. Frankfurt a. M. 2001, S. 57.

${ }^{150}$ DeVore, Irven: Foreword. In: Barbara B. Smuts: Sex and Friendship in Baboons. New York 1985, S. xi-xiii, hier S. xi, Hervorheb. MS.

${ }^{151}$ DeVore: Foreword, S. xii.
} 
DeVore spricht von ,proximity“, von ,intimate exchanges“ und ,intimate relationships“, 152 von einer Ethnologie-analogen Rekategorisierung der Tiere von ,subjects ‘ zu ,informants', die Smuts' Studie gegenüber jenen privilegiere, die in der wissenschaftlichen Revolution in der Verhaltensökologie Mitte der 1970er Jahre durchgeführt worden waren. In jener Rekonfigurierung des theoretischen Feldes war es ,necessary to ,objectify “ the animals and their behavior in order to purge primate studies of the easy anthropomorphism of the turn of the century“, ${ }^{153}$ heißt es hier. Doch die ,powerful predicitive theories“154 der Soziobiologie konnten das Pavianverhalten anders als erhofft nicht restlos aufschlüsseln und erklären: ,[N]ew methods were needed that allowed observers to focus on those behaviours that were most meaningful to the animals themselves, and new theories had to be developed to explain the often surprising findings that emerged". 155

Barbara Smuts selbst dankt in ihren „Acknowledgments“ vier ,people who played a central role in my training“ $(S F, \mathrm{xv})$. Neben Robert „Bob“ Trivers, Irven „Irv“ DeVore und dem ebenfalls soziobiologisch argumentierenden Zoologen Robert Hinde wird hier auch der Psychiater David Hamburg genannt, der bereits das Vorwort zu Jane Goodalls In the Shadow of Man beigesteuert hatte. ${ }^{156}$ Dessen „,belief in the evolutionary significance of emotions and enduring social bonds“ $(S F, \mathrm{xv})$ wird als für die Arbeit inspirierend ausgewiesen. ${ }^{157}$ Damit setzt Smuts bereits subtil einen anderen Schwerpunkt als Hrdy.

Im Zentrum von Smuts' Studie Sex and Friendship and Baboons steht die Funktion von ,Freundschaft", genauer ,long-term, cross-sex ,friendships““ (5) als Sozialverhalten von Pavianen. Smuts' Forschungsarbeit fragt also von einem evolutionsbiologischen Standpunkt aus danach, welche Rolle soziale Beziehungen zwischen männlichen und weiblichen Pavianen spielen, die nicht allein auf

\footnotetext{
${ }^{152}$ Alles DeVore: Foreword, S. xii. Diese Qualität von Smuts' Primatologie hebt auch Haraway hervor, vgl. Haraway: When Species Meet, S. 23 ff.

${ }^{153}$ DeVore: Foreword, S. xii.

${ }^{154}$ DeVore: Foreword, S. vii.

${ }^{155}$ DeVore: Foreword, S. xiii, Hervorheb. MS.

${ }^{156}$ Siehe Hamburg, David: Foreword. In: Jane van Lawick-Goodall: In the Shadow of Man. Boston Company 1971, S. xv-xvii.

${ }^{157}$ Der Einfluss einer aufkeimenden neuen Emotionsforschung geht jedoch noch weiter: Frans de Waal, dessen Forschungsinteressen sich von der Politik über die Moral bis zur Empathie und anderen Emotionen unter Primaten entwickelt haben, erhält eine Nennung in Bezug auf die abgebildeten Fotographien. Weitere Erwähnung erhalten u. a. Lila Abu-Lughod und Victoria „Vicki“ Burbank, die beide mit sozialanthropologischen Studien zu Emotionalität bekannt wurden. Siehe Abu-Lughod, Lila: Veiled Sentiments: Honor and Poetry in a Bedouin Society. Berkeley 1986; Burbank, Victoria: Passion as Politics: Romantic Love in an Australian Aboriginal Community. In: William Jankowiak (Hg.): Romantic Passion: A Universal Experience. New York 1995, S. 187-195; und für de Waal zuletzt: de Waal, Frans: The Age of Empathy: Nature's Lessons for a Kinder Society. New York 2009; Ders.: Mama's Last Hug. Animal Emotions and What They Tell Us About Ourselves. New York/London 2019.
} 
sexuelle Kontakte beschränkt oder durch Verwandtschaftsverhältnisse erklärbar sind. Sie beschäftigt sich mit der evolutionären Funktion eines sozialen Investments: „Has natural selection favored friendship among baboons? Specifically, does having a friend of the opposite sex help an individual to maximize his or her genetic contribution to future generations?“ (7); ,how do friendships contribute to individual reproductive success?“ (8). Dabei beruft sich Smuts grundsätzlich auf jene ,powerful theories for explaining behavior developed by evolutionary biologists over the last 100 years" (7), von denen auch DeVore schon sprach. Es geht ihr jedoch auch um die Vereinbarkeit von ,scientific scrutiny and objective communication" mit ,the immediacy and vividness of the raw observations" (8), dem „,daily drama of baboon life“ (8). Im ,interplay“ (9) der genauen Beobachtung mit der theoretischen Grundlage für die Interpretation dieser Beobachtungen in einem „evolutionary framework“ ist es ausdrücklich Smuts' Ziel, „to convey a feeling for the process, as well as the results, of the study of primate behavior" (9, Hervorheb. MS).

In der Tradition des Genres beginnt Smuts nach den einführenden Bemerkungen mit Informationen zur Spezies und deren Habitat und Sozialverhalten, sowie genaueren Angaben zur untersuchten Eburru-Cliffs-Truppe im zweiten Kapitel. Das dritte Kapitel umfasst unter dem Titel „Field Work and Data Analysis“ (27 ff.) Anmerkungen zur Habituation der Paviangruppe, zur Identifizierung einzelner Individuen, zu den Methoden der Datensammlung, zur Art der Daten - quantitativ und qualitativ - und zur Präsentation der Ergebnisse im vorliegenden Text. Das darauffolgende Kapitel ist ganz der Definition des Freundschaftsbegriffs gewidmet. Graphen, Tabellen und sogar Formeln dienen hier der Illustration eines wissenschaftlich abgesicherten Begriffs von Freundschaft und somit als Bollwerk gegen die Vermutung, hier sei ,einfach` anthropomorphisierend ein Begriff aus dem menschlichen Sozialleben auf die Paviane übertragen worden. So abgesichert kann Smuts dann zu Beginn ihres fünften Kapitels (,What Made Friends Special“, 60 ff.) behaupten:

\footnotetext{
What made Friends special was, most of all, the unusual quality of their interactions. Female baboons, in general, are wary of males. This is understandable: Males sometimes use their larger size and formidable canines to intimidate and bully smaller troop members. Females, however, were apparently drawn to their male Friends, and they seemed surprisingly relaxed around these hulking companions. The males, too, seemed to undergo a subtle transformation when interacting with female Friends. They appeared less tense, more affectionate, and more sensitive to the behavior of their partners. (61)
}

Es fällt auf, dass die Einführung in das Thema Freundschaft mit einem Hinweis auf affektive Zustände der Tiere (,,apparently drawn to“, „relaxed“, „less tense“, „more affectionate“, „more sensitive“) als ,Ergebnis“ des freundschaftlichen Verhältnisses verbunden wird. An anderer Stelle geht Smuts auf den ,,intimate tone of interactions between Friends and infants" (110) oder die ,affectionate quality of the majority of interactions between male Friends and infants" (111) ein, welche sie als „striking to the observer but difficult to describe“ (111) bezeichnet. Später im selben Kapitel gibt Smuts als „most striking result“ eines Vergleichs von 
„Friends and Non-Friends“ hinsichtlich ihres Grooming-Verhaltens ,the females' apparent desire to be near their Friends“ (76) an. Dieses Begehren wird im nächsten Kapitel jedoch bereits im Titel mit der soziobiologischen Frage nach den „,benefits“ eines solchen Verhaltens kombiniert („Benefits of Friendship to the Female“, 80 ff.): „Male protection and infant care“ sind ,important rewards that motivate females to form friendships“ (81).

Hier werden nun auch Beispiele aus der Feldbeobachtung herangezogen, in denen die Tiere namentlich benannt werden. Smuts kann offensichtlich im Rahmen ihrer Arbeit unbedenklich ein emotionales Leben der Tiere in Betracht ziehen. Eine unprovozierte Attacke von Männchen auf Weibchen lässt Letztere z. B. „often literally shaking with fear and choking on her own screams“ (93); ,[20 min later] I found her still huddled under the bush, her head buried in her arms. As far as I could tell, she was not injured in the attack - just very frightened" (93). Diese Hinweise auf tierliche Emotionalität werden dem Bemühen zugeordnet, einem beobachteten Verhalten auf den Grund zu gehen, das anderweitig - allein durch ökonomische Kosten-Nutzen-Rechnungen im Dienste reproduktiver Gewinnmaximierung - nicht ergründbar ist:

These prolonged attacks puzzled me for a long time. Why should an apparently unprovoked male expend so much effort in order to terrify a female one-half of his size? I sometimes wondered if perhaps these incidents were simply irrational acts by males who had temporarily ,lost control of their emotions " - a disquieting and unsatisfying conclusion for someone who expects the regular behavior patterns of wild animals to make sense. (93)

Es geht Smuts aber auch nicht darum, Emotionalität als Opposition des ökonomisch Rationalen (,irrational acts“, „lost control“) zur normativen Erklärung heranzuziehen. Erst die Langzeitbeobachtung rückt die Attacken in einen komplexen sozialen Rahmen. Dies ,are attacks in response to specific acts by the victim that the attacker observed and remembered; it is the observer who does not know the reason for the male's behavior, not the baboons“ (96). Die Angriffe, so stellt sich heraus, sind Strafaktionen von Männchen gegen Weibchen, die sich einem anderen mit dem Männchen befreundeten Weibchen gegenüber feindlich verhalten haben oder die mit einem anderen Männchen befreundet sind, das sich gegenüber einem befreundeten Weibchen aggressiv verhalten hat. Freundschaften, so stellt Smuts fest, sind sowohl vorteilhaft als auch kostspielig:

[A]lthough Friendship with males provides females with an important benefit - defense against aggression by other baboons - these relationships may also impose costs. The potential disadvantages of friendship include becoming a focus of redirected aggression by a male involved in an agonistic encounter with the Friend, being used by another male as a means of directly challenging the Friend, and, finally, being used by the Friend $[\ldots]$ to manipulate the outcome of a tense or aggressive interaction with another male. (101)

Smuts zeichnet hier das Bild eines komplexen und voraussetzungsreichen sozialen Verhaltens, das durch die einfache Kosten-Nutzen-Aufteilung der Soziobiologie 
allein nicht (reproduktions-)strategisch erklärt werden kann. Die „multiple, complex, and interrelated sets of social tactics and social relationships that must be considered" (102) bilden zusammengenommen ein Verhaltensmuster, das erstens eine längerfristige und zweitens eine emotionale Strategie ist:

The preceding analysis suggests that females form friendships in part because of their extreme vulnerability to males in general. A female baboon is continuously surrounded by males who could quite easily injure, or even kill, her or her offspring. Her most effective defense against these dangers is another male. But rather than relying on the possibility that some male will risk injury and come to her aid, the female forms a long-term bond with one or two males who are more reliable allies, presumably because they in turn receive benefits from their relationship with her. [...] [F]riendship has evolved as a result of female vulnerability to aggression from other troop members and perhaps, especially, from males. (102, Hervorheb. im Original)

Ähnliches lässt sich in Smuts’ Bericht in Bezug auf die „Benefits of Friendship to the Male“ (Kap. 8, 159 ff.) beobachten, wenn es darum geht, dass die Pavianweibchen ihre, female choice" trotz ihrer körperlichen Unterlegenheit durchsetzen können. „,[A]cquiring a consort partner“ ist für Männchen nur ,the first step toward mating success. Whether or not the male derives a reproductive benefit from acquiring a partner depends on the female's behavior“"(172). In Smuts' Beobachtungen können sich die Weibchen nicht nur der Kopulation verweigern, indem sie nicht kooperieren (z. B. indem sie weglaufen, sich verstecken, sich hinsetzen etc.). Sie können auch ein „,consort turnover“ (172) (Wechsel der Partner/ innen) herbeiführen. Dies geschieht durch Demotivation des um die Kopulation bemühten Männchens, oder:

[B]y refusing to cooperate with her partner, the female may signal to other males her lack of preference for him. This information, in turn, can have an important effect on another male's willingness to challenge the consort relationship [...]. Thus, even if a female's attempts to approach other males or to escape from her partner do not result directly in a turnover, these highly visible behaviors may affect the likelihood of a future turnover by informing other males of her preferences and aversions. (172)

Mit einem Weibchen befreundete Männchen zeigen sich eher bereit, in einem solchen Szenario ein ,turnover' anzustreben, als nicht-befreundete.

Indem Smuts den Blick auf Weibchen und deren freundschaftliche Beziehungen zu Männchen richtet, findet sie erstens die Figur eines primatischen Weibchens, das aus der körperlichen Unterlegenheit heraus zur politischen Strategin wird, die soziale Bande auf lange Sicht hin knüpft, um zuallererst sich, aber auch ihren Nachwuchs zu schützen. Dadurch werden Weibchen zu mitgestaltenden Akteurinnen im sozialen Gefüge. Dies wird für Smuts vor allem dort sichtbar, wo es um das Ausüben der ,female choice', also im Wesentlichen die Präferenz für Sexualpartner, geht: 
[F]emale baboons do not simply acquiesce to the outcome of male-male competition. By expressing their mating preferences, females alter the costs and benefits of aggressive competition among males and thus influence the form and frequency of male-male competition (180). ${ }^{158}$

Zweitens macht sie hier eine emotionale Dimension - ,, a long-term bond“, ,,affection" etc. - als wesentlichen Teil dieser Strategie sichtbar. DeVore hatte in seinem Vorwort bereits darauf verwiesen, dass es sich bei den Pavianen um ,,intelligent, curious, emotional and long-lived creatures" 159 handle. Smuts stellt den emotionalen Bereich jedoch nicht nur als Eigenschaft der Art, sondern als strategisches Kapital heraus. Ein solches Kapital ist Emotionalität sowohl im strategischen Sozialverhalten der Tiere als auch als Gegenstand für die primatologische Forschung. Dies wird explizit gemacht, wenn Smuts „Emotions underlying Sex and Friendship“ (220) untersucht und dafür plädiert „to shift levels of analysis. We need to consider motivations: changes in internal states that direct the individual toward some goal that will decrease negative feelings (e.g. hunger, fear, loneliness) and/or increase positive ones (e.g. satiation, security, sexual pleasure)“ (220). Emotionalität, hier verstanden als ,,[m]otivations and the subjective, emotional states that accompany them“ (220), sind bei Smuts ,mechanisms that have evolved through natural selection to make individuals behave in ways that, on average, increase reproductive success“ (220 f.). In diesem letzten Teil der Monographie, auf den die vorangegangenen Ausführungen zulaufen, plädiert Smuts für ein neues Augenmerk auf emotionale Zustände als Motivatoren bei Tieren. Gegen die Angst vor dem Anthropomorphismus-Vorwurf, der mit der Thematisierung von Emotionen bei Tieren traditionell einher geht (vgl. Abschn. 4.1.1), setzt sie die programmatische Notwendigkeit, Fragen zum tierlichen Sozialleben zu klären, die sich nicht allein durch die Kalkulationen der Soziobiologie erklären lassen:

When we refuse to use our inferential skills to investigate animal emotions, we forgo knowledge of the forces that give animals personality and vitality; we deny them the spirit that has captured our interest to begin with. This seems a large price to pay for the certainty and safety of ,objective“ knowledge. (221)

\footnotetext{
${ }^{158}$ Smuts entwickelt auch ein vergleichbares Modell des Vorteils des sozialen Investments für ,Männerfreundschaften“: Männchen „,derive important benefits from the development of stable, cooperative relationships with other males. [...] [S] uch relationships are most likely to survive when each partner follows the ,tit-for-tat" strategy, responding to an altruistic act with another altruistic act and responding to defection (an act that inflicts a cost) by defecting also" (SF, S. 147). Zudem versieht sie auch Männchen mit strategischem Verhalten. Die Freundschaft mit Weibchen hat nicht nur reproduktive Vorteile; Männchen sind zudem ,able to use both female Friends $[\ldots]$ and those females' infants $[\ldots]$ as buffers during agonistic encounters with other males“ (S. 198). In Smuts' Ausführungen wird zunehmend deutlich, dass es gerade die Männchen sind, welche stark von Freundschaften mit Weibchen profitieren - angefangen bei der Intergration von migrantischen Männchen in die neue Gruppe über den Zugang zu Ressourcen (Essen, Sex) bis hin zum Schutz vor Aggression.
}

${ }^{159}$ DeVore: Foreword, S. xiii. 
Es gebe, so Smuts, Verhaltensformen, ,that seem best described in terms of familiar human emotions“ (221). Unter Zwischentiteln wie „Ambivalence“ (222), „Flirtation and Courtship“ (223), „Jealousy“ (223), „Trust“ (226), „Affection“ (227) und „Grief“ (229) versammelt Smuts Beispiele aus der Feldbeobachtung, die auf subjektive emotionale Zustände bei den Tieren hinweisen. Smuts kommt schließlich zu einem geradezu bahnbrechenden Schluss:

[B]aboons show evidence of emotions toward their friends and mates that seem to resemble feelings that people experience in similar contexts. These emotions, rather than a compulsion to out-reproduce others, are the immediate cause of the interactions and relationships that make up the fabric of their social lives (231, Hervorheb. MS).

Obwohl Barbara Smuts soziobiologisch formuliert und ökonomisch analysiert, sieht sie hier die Motivation durch Emotionen und nicht den bis auf die Ebene der Gene hinunterreichenden Wettbewerb um die Reproduktion als geeigneten Schlüssel für primatisches Sozialverhalten.

In einem letzten Kapitel vergleicht Smuts ihre Ergebnisse mit Studien anderer primatischer Arten hinsichtlich der Evolution von zwischengeschlechtlichen Beziehungen. Es geht ihr - in einer abschließenden Rechtfertigung der Forschungsarbeit - um die Übertragbarkeit auf die menschliche Gattung. Dabei wird Smuts' theoretische Neuordnung noch einmal deutlich. Denn sie distanziert sich vom Modell des „economic exchange between the sexes“ (259) zur Erklärung für „the emergence of highly differentiated, reciprocal bonds among ancestral males and females“ (259). Smuts lehnt dabei jedoch nicht grundsätzlich soziobiologische Erklärungsmodelle ab, die auf einer Kosten-Nutzen-Rechnung des Verhaltens basieren. Es geht ihr schlicht darum, dass es nicht nur ökonomische und reproduktive ,benefits“ geben kann, sondern auch ,important social benefits“ (260) geben muss, die erst die Grundlage für die Möglichkeit eines ökonomischen Erklärungsmodells liefern. Sie setzt nicht ,den Markt' als Grundlage für Affekte an, sondern affektive Bindungen für die Einrichtung ökonomischen Austausches voraus:

If this hypothesis is correct, then the economic exchange engendered by a division of labor probably developed within the context of male-female relationships that had already existed for a very long time. [...] [I]t was possible for male-female food-sharing to develop because females and males had already evolved the capacity for close bonds that allowed them to successfully negotiate reciprocal exchange. (260)

Barbara Smuts grundiert somit mit ihrem Sex and Friendship in Baboons den homo oeconomicus im Rahmen soziobiologischer Theorie durch die Arbeit mit dem animale oeconomicum affektiv.

\subsubsection{Von Strategien des Egoismus zur emotionalen Agency}

An Sarah Blaffer Hrdys und Barbara Smuts' Texten lässt sich exemplarisch nachverfolgen, wie unter dem Einfluss neuer theoretischer und gesellschaftspolitischer Paradigmen in einer neuen Episteme der Primatologie Emotionalität auf der Ebene 
der Tiere selbst verhandelt wird. Welche Rolle Emotionen in der Forschung - vor allem als Gegenstand - spielen können und welche Erklärungsmuster zulässig sind, werden durch die expliziten oder impliziten Regeln emotionaler Regime des Faches und der Zeit vorgegeben.

In diesem Fall lässt sich nicht von einem neoliberalen Umgang mit Emotionalität in der primatologischen Forschung sprechen, sondern es muss im Sinne jener Differenzierungen, die bereits Fleck, Rosenwein, Daston und Koch vorgenommen haben (vgl. Abschn. 4.1.4), von mehreren Ebenen und Phasen ausgegangen werden. Sarah Blaffer Hrdys The Langurs of Abu zeigt, wie die Implementierung eines neueren Theorieansatzes, der mit einem ökonomischen Grundmodell durchdringend kooperiert, als starkes, einhegendes Korsett wirken kann. Es ermöglicht derart von der Forschung mit Affen zu berichten, dass Emotionen kaum eine Rolle spielen. Niemand würde in Abrede stellen, dass die 1960er und 1970er Jahre Emotionen kannten oder dass sie mit hochaffektiven Ereignissen besiedelt waren. Ebenso wenig lässt sich unterstellen, dass die Arbeit an und mit Emotionalität in der primatologischen Feldforschung, die in der Episteme der ,Trimates` zutage gefördert und kultiviert wurde, Hrdy und ihren wissenschaftlichen Zeitgenoss/innen unbekannt war. Doch die Gefühlsregeln des neuen, stark theoriegeleiteten Regimes der Soziobiologie drücken - mit Arlie Hochschild und Gertraud Koch - eine Ideologie aus, die zeitgenössisch mit Erklärungsmodellen arbeitet, die Emotionalität in der Forschung im Text, wegrationalisiert'. Emotionales Management bedeutet hier zunächst einmal: Die in der Begegnung von Gender- und fachlichem Diskurs gerade im Empathie-Begriff problematisch gewordene Konnotation von Emotion und Weiblichkeit kann dank der neuen, von einer feministisch inspirierten Primatologie bereitwillig übernommenen Ansätze einer biologisch-ökonomisch operierenden Evolutionstheorie umgangen werden. Um das Verhalten von genprogrammierten Fleischmaschinen zu verstehen, braucht es kein empathisches Verständnis, sondern den Nachvollzug strategischer reproduktiver Kalkulationen. Perspektivübernahmen sind hier keine empathischen Einsichten, sondern abstrahierende Verallgemeinerungen. Weil Emotionalität in einem solchen Verständnis keinen Platz hat, muss sie auch nicht benannt, differenziert, gerechtfertigt oder erläutert werden. Barbara Smuts' Sex and Friendship hingegen zeigt fast zehn Jahre später auf, dass in der nächsten Phase und in der Entwicklung der soziobiologischen Grundlagen Emotionen wieder in Erscheinung treten können. Durch die Kombination von soziobiologischen Erklärungsansätzen und einer Aufwertung von Emotionalität als Ergebnis und Grundlage der Wertschöpfung in einem psychologisch-therapeutischen Diskurs und in neuen sozioökonomischen und arbeitsorganisatorischen Ansätzen werden sie zu Motivatoren und Quellen für primatisches Verhalten. Ganz ohne die Thematisierung von Emotionalität in der Forschung (seitens der Forschenden) werden Affekte so als Ressource für die Forschung vorbereitet.

Es zeigt sich, ähnlich wie Reddy dies für seinen Forschungsgegenstand vermutet hat, dass das Erbe und die emotionalen Praktiken der frühen Feldforschung nicht verschwunden sind, sondern in neuen theoretischen Rahmen (der Soziobiologie in Kooperation mit einer zunehmend biologisch fundierten Ökonomie) und 
unter einem neuen epistemischen Regime (des sich verfestigenden Neoliberalismus als neuem ,Geist des Kapitalismus') mit seinen eigenen Gefühlsregeln (die besonders in der feministisch inspirierten Primatologie zum Tragen kommen) innerhalb der wissenschaftlichen Gemeinschaften neu konfiguriert werden und zu anderem Nutzen kommen.

\subsection{Darting the Inner Postironic: Ironie und Immunität in Robert Sapolskys A Primate's Memoir (2001)}

\subsubsection{Humoristische Primatologie}

„I joined the baboon troop during my twenty-first year. I had never planned to become a savanna baboon when I grew up; instead I had always assumed I would become a mountain gorilla“ $(P M, 13)$. So beginnt Robert Sapolsky über zwei Dekaden nach dem Erscheinen von Sarah Blaffer Hrdys The Langurs of Abu und Barbara Smuts' Sex And Friendship in Baboons seinen deutlich literarisierten autobiographischen Forschungsbericht A Primate's Memoir. Wie schon diese ersten Formulierungen (und die bereits angeführten Passagen zu seiner Reise in die Virungas) zeigen, spielt Sapolsky mit dem uneigentlichen Sprechen. Natürlich hält sich Sapolsky trotz seines neurologischen Interesses an Psychosen und Schizophrenien weder für einen Savannenpavian noch für einen Berggorilla. Doch er weiß mittels dieser ersten Sätze einen Erzählton zu setzen, der auf humoristische Weise die Umstände der Feldforschung ebenso wie die Probleme des Forschenden vermittelt: Um die Tiere beobachten zu können, nähert sich Sapolsky der Gruppe wie ein migrierendes junges Pavianmännchen. Durch die anschließende Habituation und die langjährige Forschung sind die Paviane für Sapolsky familiäre Bekannte geworden. Obwohl er eigentlich Berggorillas beforschen wollte, hat sich sein wissenschaftliches Interesse in eine Richtung verschoben, in der er zum Pavian(-forscher) wird.

In Sapolskys Text ist fast jeder primatologische Sachverhalt humoristisch formuliert. So ist z. B. das Abwandern der Jungmännchen aus der Heimatgruppe, wenn die Männchen ,get this undefined itch of wanderlust“, „one of those incest avoidance deals“ (17). Das Ziel genzentrierter Evolution ist: „leaving as many copies of your genes in future generations as possible, all that jazz" (18, Hervorheb. MS). Auch Sapolskys Beschreibungen des Pavianverhaltens unterliegen der Komik. Begrüßungsrituale werden beispielsweise wie folgt beschrieben: „When male baboons who are getting along well run into each other and want to say howdy, they yank each other's penises. [...] All the guys did it to the other guys that they were pals with“ (18). Saloppe, umgangssprachliche Formulierungen (,,all that jazz", „howdy“, ,guys“; ,,[n]o dice“, „ol’ Aaron“, ,poor Ruthy was in hormonal limbo and going bonkers"; alles 19) sind dabei ebenso stilistische Mittel wie rhythmisierende Ellipsen: „Only females I ever saw do it“ (18); „Quiet kid, didn’t make trouble, had a serious, unflappable look about him. Masturbated a lot in the bushes“ (19). Teilweise streut Sapolsky jiddisches Vokabular ein und ironisiert 
wiederum zugleich dessen Gebrauch: „To use technical lingo, Naomi's family were mensches, and they soon became my favorite lineage" (22, Hervorheb. MS). Sapolsky schafft sich mit diesen und anderen Mitteln eine nahbare, räumlich (Brooklyn, New York) und kulturell (orthodoxes New Yorker Judentum) verortbare Erzählstimme, die seiner Leserschaft auf überwiegend nicht ganz ernste Art und Weise seine Themen vermittelt.

Dabei sind die Paviane und die Feldforschung nicht Sapolskys einziger Fokus. Zwar gibt die Forschungssituation, die Paviangruppe in Kenia im Feld zu beobachten, den Rahmen des Textes vor. Er ist jedoch stärker als andere Forschungsmemoiren auch in einer anderen Hinsicht autobiographisch: Die vier an ethologische Lebensalter-Kategorien angelehnten Teile „The Adolescent Years“ (11 ff.), „The Subadult Years“ (93 ff.), „Tenuous Adulthood“ (167 ff.) und „Adulthood“ (231 ff.) ordnen nicht nur die Anekdoten über die im Fokus stehenden Männchen der beobachteten Gruppe, sondern formen auch narrativ Sapolskys Heranwachsen als Primatenforscher. Von den Anfängen Ende der 1970er Jahre als ,Grad Student “ über die relative Unabhängigkeit als ,Post Doc' bis zur Erzählgegenwart als ,Tenured Professor' zeigt A Primate’s Memoir, wie Sapolsky durch diese Forschungsaufenthalte in Kenia als Forscher und durch seine Reisen durch Ostafrika als Mensch gebildet wird. In diesem Sinne orientieren sich seine Forschungsmemoiren am Format des Bildungsromans. ${ }^{160}$ A Primate's Memoir ist dabei jedoch keine stringente Erzählung von der Ankunft des naiven Doktoranden in der Masai Mara bis zur gereiften Gegenwart auf einem Lehrstuhl. Stattdessen bedient sich Sapolsky eines anekdotischen Erzählens, das immer wieder andere Aspekte berücksichtigt und verschiedene Perspektiven einnimmt: Er ist Neuroendokrinologe und Primatenforscher in der Sicht auf die Paviane, Anthropologe und Kulturbotschafter in seiner Nachbarschaft mit den einheimischen Masai, Tourist und Abenteurer auf seinen Reisen durch Kenia und die angrenzenden Staaten, sowie Historiker und Berichterstatter in seiner Beschäftigung mit der Geschichte und Politik Ostafrikas. Die Autobiographieforschung hat Sapolskys Memoir aufgrund dieser Vielfalt bereits entdeckt. Sie hat versucht, genau diese Vielfalt psychoanalytisch als ,coping mechanism' eines gespaltenen Selbst zu lesen, das die Ansprüche wissenschaftlicher Objektivität mit der Praxis der Feldforschung und der Fremdheitserfahrung des Amerikaners in Afrika zu vereinbaren sucht. ${ }^{161}$

\footnotetext{
${ }^{160}$ Diese literarische Orientierung ist kaum zufällig: Avanessian weist darauf hin, wie nah sich Bildungsroman und Autobiographie darin sind, Spezialfälle ironischer Verfahrensweisen und Gattungen zu sein: „Egal ob unfreiwillig epigonisch oder freiwillig parodistisch - in beiden Gattungen lassen sich die Spannungen nicht hintergehbarer Ironizität leicht verifizieren, wobei sich die Frage nach Autor und Held in vielfacher Weise überkreuzen und überdecken. Offensichtlich ist das im Fall der Autobiographie. Aber auch für den Bildungsroman gilt tendenziell, dass die Probleme seiner Figuren die Problematik der jeweiligen Autoren verdoppeln“. Avanessian, Armen: Phänomenologie ironischen Geistes. Ethik, Poetik und Politik der Moderne. München 2010, S. 230.

${ }^{161} \mathrm{Vgl}$. Forbes, Shannon: The Intersection of Narrative and Science in Robert Sapolsky's A Primate's Memoir. In: a/b: Auto/Biography Studies 26/2 (2011), S. 323-341, hier S. 325 ff.
} 
Doch die psychoanalytische These des ,split self' und seiner vielen Identitäten scheint mir zu verkennen, dass Sapolsky dieser Perspektivwechsel und der vermeintlich distanzierende ironische Modus nicht einfach unterlaufen. Beides setzt Sapolsky m. E. als Autor gezielt und im Sinn einer (post-)ironischen Postmoderne ein, auf die Sapolsky subtil anspielt. Bereits in seinen „Acknowledgments“ (9 f.) verweist er beispielsweise nicht nur auf „Creative Writing 101“ $(10)^{162}$, sondern auch darauf, manche Figuren seiner Memoir seien Komposita mehrerer Individuen. Die „major baboon figures“ und „major human characters“ jedoch „are real individuals“ und „I, to the best of my knowledge, am not a composite“ (9). Diese ironische Herangehensweise an die (post-)moderne Ich-Problematik lässt vermuten, dass Sapolsky sich der Verfahren postmodernen Schreibens und der sie begleitenden Theorien sowie der Qualität seiner Memoir als literarischer und zugleich wissenschaftsautobiographischer Text bewusst ist. ${ }^{163}$ In einem Interview nennt er den Text - auch wieder ironisch - sogar ,this epic schlocky novel sort of thing". 164

Im Folgenden soll nun jedoch keine Analyse der Textstrategien der Postmoderne an Sapolskys Text nachvollzogen werden. Damit ließe sich der Text nur im poetologischen Diskurs seiner Zeit einordnen. Stattdessen geht es mir um den Zusammenhang von Sapolskys Ironie und seiner Thematisierung von Emotionalität in einer Zeit, in der Ironie als (auch affektiv) problematisch diskutiert wird. Mit dem Begriff ,Ironie“ soll hier der humoristische Stil ebenso wie das uneigentliche Sprechen und, in Northrop Fryes Verständnis jener „mythical patterns of experience, the attempts to give form to the shifting ambiguities and complexities of unidealized experience" ${ }^{165}$ der ironisierende Umgang mit bestimmten Themen gefasst werden. Ironie ist bei Sapolsky nicht nur eine Haltung, mit den Ambiguitäten und Komplexitäten erfahrener Wirklichkeit umzugehen, also im Sinne der

\footnotetext{
${ }^{162}$ Sapolsky dankt seinen Lektorinnen dafür, ihn auf Probleme hingewiesen zu haben, ,that anyone but a scientist should have learned back in Creative Writing 101“ (PM, S. 10) - ein Seitenhieb auf den solipsistischen Stil mancher (natur-)wissenschaftlicher Texte.

${ }^{163}$ Zum dieser Vermutung zugrunde liegenden Konzept postmoderner Literatur vergleiche McHale, Brian: Postmodernist Fiction. London/New York 2004; Hutcheon, Linda: A Poetics of Postmodernism. History, Theory, Fiction. New York/London 1988; Lodge, David: The Modes of Modern Writing. Metaphor, Metonymy, and the Typology of Modern Literature. London 1977.

${ }^{164}$ Bacon, Katie: Of Monkeys and Men: An Interview with Robert Sapolsky. In: Atlantic Unbound. The Atlantic Online, April 2001, https://www.theatlantic.com/past/docs/unbound/interviews/int2001-04-25.htm (16.04.2018). Als jiddisches Lehnwort im Englischen verweist, schlocky" umgangssprachlich auf die mangelnde Qualität eines Gegenstandes. Siehe schlock, n. In: OED Online (2018), https://www.oed.com/view/Entry/172432 (02.01.2018).

${ }^{165}$ Frye: Anatomy of Criticism, S. 223. Fryes Systematisierung literarischer Genres ist nicht postmodern, in dieser Formulierung wird aber ein andauerndes Verständnis von Ironie als Form der Erfahrungsbewältigung transportiert, das für die Postmoderne ebenfalls geltend gemacht werden kann.
} 
Ironieforschung ein Ethos, das sich bis auf Sokrates zurückführen lässt. ${ }^{166}$ Ironie ist ihm eine umfassende Strategie, mit Emotionalität in der Feldforschung umzugehen, sie sichtbar werden zu lassen und für ihren Wert in der individuellen Forschungspraxis einzustehen. Die ironisierenden Verfahren lassen sich daher, so Annahme dieses Kapitels, als Teil eines Affektregimes verstehen, das es Sapolsky ermöglicht, auch intensive Emotionalität in der Primatologie verhandeln zu können.

Im Folgenden soll zunächst in den Ironiediskurs um die jüngste Jahrhundertwende eingeführt werden, aus dem heraus sich der Stellenwert von Ironie bei Sapolsky verstehen lässt (Abschn. 4.3.2). Es ist besonders die darin diskutierte Doppelfunktion von Ironie als Ethos und als Strategie, die Sapolskys fruchtbar macht. Anschließend werde ich mich damit auseinandersetzen, wie und vor allem wozu Sapolsky Ironie strategisch verwendet (Abschn. 4.3.3). Es lässt sich zeigen, wie Sapolsky sich ironisch von seinem jüngeren Ich distanzieren kann, ohne dessen Anteil an seiner Selbstformierung zu negieren; wie es ihm die Ironisierung ermöglicht, Normen und Praktiken zu kritisieren, die mit seinem wissenschaftlichen Feld und seinem Forschungsvorhaben in Zusammenhang stehen; und schließlich, dass Sapolsky auf ,Schutzironie' (Wolf) zurückgreift, um das Schreiben von unzeitgemäßen , vehementen Passionen' (Fisher) zu legitimieren. ${ }^{167}$ Schließlich soll damit aufgezeigt werden, welches kritische Potenzial in Sapolskys Ironie steckt - und dabei auf Abstand zum ,postironischen" Vorwurf der ,zahnlosen Ironie' gegangen werden. Die übergreifende These hierbei ist, dass der strategische Einsatz von ironischen Verfahren Sapolsky in A Primate's Memoir dahingehend immunisiert, ${ }^{168}$ Emotionalität nicht nur thematisieren und Anthropomorphismen ausführlich ausgestalten (Abschn. 4.3.4) zu können, sondern bestimmte Affekte auch als evaluierendes Sensorium einer Ethik auszuzeichnen, die letztlich Fragen zur Verantwortung für die Forschungstiere und die eigene Forschungspraxis aufwirft.

\footnotetext{
${ }^{166}$ Vgl. Colebrook, Claire: Irony. London/New York 2004, S. 2; Hutcheon, Linda: Irony's Edge. The Theory and Politics of Irony. London/New York 1995, S. 66.

${ }^{167}$ Siehe Wolf, Werner: ,Schutzironie‘ als Akzeptanzstrategie für problematische Diskurse: Zu einer vernachlässigten Nähe erzeugenden Funktion von Ironie. In: Thomas Honegger/Eva-Maria Orth/Sandra Schwabe (Hg.): Irony Revisited. Spurensuche in der englischsprachigen Literatur. Würzburg 2007, S. 27-50; Fisher, Philip: The Vehement Passions. Princeton/Oxford 2001.

${ }^{168}$ Der Begriff Immunität wurde von Martin von Koppenfels bereits in die literaturwissenschaftliche Emotionsforschung getragen. Anders als bei von Koppenfels soll Immunität im vorliegenden Kontext jedoch nicht als „Gefühl der Gefühllosigkeit“ oder „Nichtgefühl“ verstanden, sondern in einem älteren, juristischen Sinn eines Schutzes vor Belangung oder Strafverfolgung (politische Immunität) genutzt werden. Siehe von Koppenfels, Martin: Immune Erzähler. Flaubert und die Affektpoetik des modernen Romans. München 2007, S. 11. Zum Begriffswandel der Immunität siehe auch Zumbusch, Cornelia: Die Immunität der Klassik. Berlin 2011, S. 9 f.
} 


\subsection{2 (Post-)Ironie um 2001}

\subsubsection{Diagnose: (Das Ende der) Ironie}

Robert Sapolskys Forschungsmemoiren erscheinen zu einem historischen Wendepunkt. Im Jahr ihrer Veröffentlichung forciert der 11. September eine Zeitenwende, die auch das zentrale Thema dieses Kapitels betrifft: Ironie. ${ }^{169}$ Sapolskys Memoiren stehen am Ausgang eines selbsterklärten ,Zeitalters der Ironie‘, das in einer ausgeprägten Ironie-Skepsis endet. Dies zeigt sich nicht nur in einer programmatischen anti-ironischen Schrift wie Jedediah Purdys For Common Things (1999), sondern auch in der postmodernen Ironieforschung selbst, wie Linda Hutcheons kritischer Ansatz in Irony's Edge (1995) zeigt. Dass sich seither eine Zeitenwende vollzogen hat, verdeutlichen neuere Auseinandersetzungen mit Ironie wie Claire Colebrooks Überblicksstudie Irony (2004) und Lee Konstantinous Typenstudie Cool Characters (2016), die auf die Ausrufung des ,postironischen Zeitalters` in Folge des global einschneidenden Jahres 2001 reagieren.

Sowohl Hutcheon als auch Purdy bestimmen in den 1990er Jahren Ironie als Modus des späten 20. Jahrhunderts. Sieht Hutcheon Ironie gleich zu Beginn ihrer Studie als ,a problematic mode of expression at the end of the twentieth century“, ${ }^{170}$ ist sie für Purdy „,[o]ur leading cultural currency today“ “ ${ }^{171}$ Purdys programmatische Schrift widmet sich spezifisch einer Zeitdiagnose amerikanischer Gegenwartskultur. Hutcheon geht als Postmoderneforscherin noch weiter und sieht schon in The Politics of Postmodernism (1989) eine intrinsische Verbindung von Postmodernismus und Ironie:

Postmodernism [...] takes the form of self-conscious, self-contradictory, self-undermining statement. It is rather like saying something whilst at the same time putting inverted commas around what is said. [...] Postmodernism's distinctive character lies in this kind of wholesale , nudging ' commitment to doubleness, or duplicity. ${ }^{172}$

Auch Claire Colebrook sieht Ironie als passenden Begriff für „the huge problems of postmodernity“173 und bringt die Verfahren dieser Ironie ins Spiel: ,[O]ur very historical context is ironic because today nothing really means what it says. We live in a world of quotation, pastiche, simulation and cynicism: a general and

\footnotetext{
${ }^{169}$ Als Reaktion auf die Ereignisse des ,9/11' wurde mehrfach das Ende der Ironie ausgerufen, wie The Wire und das New York Magazine anlässlich des zehnten Jahrestages feststellten. Siehe Hirschhorn, Michael: Irony, The End of. In: New York Magazine, September $9^{\text {th }}$, 2011, http:// nymag.com/news/9-11/10th-anniversary/irony/ (17.04.2018); Randall, Eric: The ,Death of Irony“, and Its Many Reincarnations. In: The Wire vom 9.09.2011, https://www.theatlantic.com/ national/archive/2011/09/death-irony-and-its-many-reincarnation/338114/ (17.04.2018).

${ }^{170}$ Hutcheon: Irony's Edge, S. 1.

${ }^{171}$ Purdy, Jedediah: For Common Things: Irony, Trust, and Commitment in America Today. New York 2000, S. 5.

${ }^{172}$ Hutcheon, Linda: The Politics of Postmodernism. London/New York 1989, S. 1.

${ }^{173}$ Colebrook: Irony, S. 1.
} 
all-encompassing irony“ “. ${ }^{174}$ Aus der intellektuellen und politischen Ironiefeindlichkeit der Zeit um 2001 schließt Lee Konstantinou, dass ,the concept of postmodernism no longer adequately described the present ${ }^{\text {“ }}{ }^{175}$ Die Postmoderne, so lautet Konstantinous Vorschlag, werde abgelöst durch die von David Foster Wallace in seinem programmatischen Text E Unibus Pluram von 1993 propagierte und durch Tendenzen der Kritik und Gegenwartsphilosophie getragene Postironie. Wallace beklagt in diesem Aufsatz ebenfalls zeitdiagnostisch, dass „irony, poker-silence, and fear of ridicule are distinctive of contemporary U.S. culture" ${ }^{176}$ Ironie und Spott seien so unterhaltsam wie effektiv, jedoch ,at the same time they are agents of a great despair and stasis in U.S. culture" ${ }^{177}$ Für Wallace ist Ironie nur einer „exclusively negative function“ ${ }^{178}$ dienlich:, ,[I]t's critical and destructive, a ground-clearing“ und dabei doch ,singularly unuseful when it comes to constructing anything to replace the hypocrisies it debunks" ${ }^{179}$ Hinter Wallace' Postironie verberge sich also, so Konstantinou, weniger ein anti-ironisches Anliegen als eine Kritik daran, dass ,irony has lost its critical power or [...] irony's critique no longer adequately addresses contemporary reality“. ${ }^{180}$

Für Purdy, Wallace und Hutcheon ist Ironie nicht mehr nur problematisch; sie ist als pathologische Erscheinung zu betrachten. Purdy sieht Ironie als Verweigerungshaltung einer seines Erachtens dringend benötigten Ernst- und Wahrhaftigkeit gegenüber, als ,,a quiet refusal to believe in the depth of relationships, the sincerity of motivations, or the truth of speech - especially earnest speech" ${ }_{1}{ }^{181}$ Sie entsteht seiner Auffassung nach aus dem Eindruck, jedes aufrichtige Gefühl sei bereits kulturell vermarktet, alles ernsthafte Sprechen vollzogen, und alles Politische unaufrichtiges Ritual geworden. ${ }^{182}$ Wie eine Krankheit muss Ironie bei Purdy

\footnotetext{
${ }^{174}$ Colebrook: Irony, S. 1.

${ }^{175}$ Konstantinou, Lee: Cool Characters. Irony and American Fiction. Cambridge, Mass./London 2016, S. x.

${ }^{176}$ Wallace, David Foster: E Unibus Pluram: Television and U.S. Fiction. In: Review of Contemporary Fiction 13/2 (1993), S. 151-194, hier S. 171.

${ }^{177}$ Wallace: E Unibus Pluram, S. 171.

${ }^{178}$ Wallace: E Unibus Pluram, S. 171.

${ }^{179}$ Wallace: E Unibus Pluram, S. 171.

${ }^{180}$ Konstantinou: Cool Characters, S. 8. Sehr ähnliche Kritik findet sich auch in der philosophischen Zeitdiagnostik des ausgehenden 20. Jahrhunderts, so deutlich bei Peter Sloterdijk, der Ironie zum Zynismus verkommen sieht, oder bei Slavoj Žižek, für den Ironie durch Institutionalisierung unwirksam gemacht wurde. Selbst dort, wo Ironie scheinbar propagiert wird, beispielsweise bei Richard Rorty, wird sie zu einer privaten, selbstformierenden Angelegenheit, die aus der öffentlichen Sphäre zu entfernen sei. Vgl. Sloterdijk, Peter: Kritik der zynischen Vernunft. Frankfurt a. M. 1983; Žižek, Slavoj: The Sublime Object of Ideology. London/New York 1989; Rorty, Richard: Contingency, Irony, and Solidarity. Cambridge u. a. 1989; Conway, Daniel W.: Taking Irony Seriously: Rorty's Postmetaphysical Liberalism. In: American Literary History 3/1 (1991), S. 198-208; Colebrook: Irony, S. 154 ff.

${ }^{181}$ Purdy: For Common Things, S. 10.

${ }^{182}$ Vgl. Purdy: For Common Things, S. xv.
} 


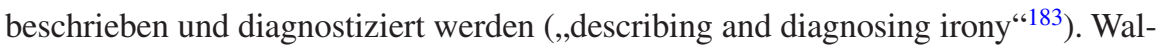
lace empfindet die Macht der Ironie als geradezu tyrannisch (,And make no mistake: irony tyrannizes us“"184). Ironie wird von Wallace mit einem zu erfolgreichen Rebellen verglichen, der die gleichen Instrumente, mit denen er den ,Feind' herausgestellt hat, nun nutze, ,to insulate itself“. ${ }^{185}$ Hutcheon fragt in ihrer Einleitung zudem ratlos: „Why would anyone want to use this strange mode of discourse where you say something you don't actually mean and expect people to understand not only what you actually do mean but also your attitude toward it?". ${ }^{186}$

\subsubsection{Die Affektivität der Ironie}

Um diese Frage zu beantworten, arbeitet Hutcheon in ihrer Studie mehrere Charakteristika der Ironie heraus, die ihre Funktionsweise beleuchten sollen: Ironie ist relational, weil sie eine (politische) Beziehung zwischen Ironiker/in und Publikum und eine Beziehung zwischen Bedeutungen stiftet. ${ }^{187}$ Sie ist dabei in gewisser Weise inklusiv, da sie nicht etwa nur als eine einfache Antiphrasis betrachtet werden sollte, sondern das Zusammenspiel mehrerer semantischer Möglichkeiten impliziert, und sie ist zugleich differenziell, da sie auf dem Erkennen von Differenz beruht. ${ }^{188}$ Hutcheon geht kritisch auf jene Funktionen der Ironie ein, die sie reizvoll machen. Sie entwirft dabei ein Modell, das von ,stumpfer ${ }^{6} \mathrm{zu}$, scharfer ${ }^{6}$ Ironie voranschreitet: ${ }^{189}$ Ironie ist ,reinforcing' in ihrem Einsatz als Emphase; ,complicating', indem sie den ästhetischen Diskurs anreichert; ,ludic' durch den spielerischen Charakter, mit dem sie eingesetzt werden kann, um zu necken und zu spötteln; , self-protective' in der Art und Weise, wie ihre selbstkritischen (,self-deprecating') Elemente gegen Verletzung schützen können; ,provisional', insofern Ironie eine Art „,built-in conditional stipulation that undermines any firm and fixed stand“190 beinhaltet; ,oppositional ' als ambivalent lesbare Instanz und , assailing ' als direkter korrektiver oder satirischer Angriff, sowie ,aggregative‘, weil sie Gemeinschaften kreiert und aus Gemeinschaften heraus kreiert wird. ${ }^{191}$ Das Problem der Ironie sieht

\footnotetext{
${ }^{183}$ Purdy: For Common Things.

${ }^{184}$ Wallace: E Unibus Pluram, S. 183.

${ }^{185}$ Wallace: E Unibus Pluram, S. 184.

${ }^{186}$ Hutcheon: Irony's Edge, S. 2.

${ }^{187} \mathrm{Vgl}$. Hutcheon: Irony's Edge, S. 17, $58 \mathrm{ff}$.

${ }^{188} \mathrm{Vgl}$. Hutcheon: Irony's Edge, S. 64.

${ }^{189} \mathrm{Vgl}$. Hutcheon: Irony's Edge, S. $48 \mathrm{ff}$.

${ }^{190}$ Hutcheon: Irony's Edge, S. 51.

${ }^{191}$ Ironie vollzieht sich für Hutcheon in diskursiven Gemeinschaften, die analog zu Thomas Kuhns Konzept wissenschaftlicher Gemeinschaften durch „the complex configuration of shared knowledge, beliefs, values, and communicative strategies" definiert sind. Anders als Wayne C. Booth, der zwischen Autor/innen und Leser/innen eine ,secret communion' (Booth, Wayne C.: The Rhetoric of Fiction. Chicago/London 1961, S. $300 \mathrm{ff}$.) und , amiable communities" (Booth, Wayne C.: The Rhetoric of Irony. Chicago/London 1974, S. 28 f.) annimmt, sieht Hutcheons diese Gemeinschaften jedoch nicht durch Ironie gestiftet, sondern Ironie funktioniert nur durch solche Gemeinschaften, die das ,Lesen ‘ von Ironie aufgrund anderer Eigenschaften bereits grundsätzlich ermöglichen. Vgl. Hutcheon: Irony's Edge, S. 91-98.
} 
Hutcheon vor allem darin, dass sie in dieser Funktionsreihung zunehmend ,,an evaluative edge“192 besitze und Emotionen hervorrufe: „,[It] manages to provoke emotional responses in those who ,get ' it and those who don't, as well as in its targets and in what some people call its , victims“". 193 Hutcheons Interesse an der affektiven Dimension der Ironie konzentriert sich auf Ironie als Bedrohung für das diskursive Wohlbefinden: „,[M]ost people feel there is something faintly (or even strongly) suspect about irony [...]. The suspicion of deceit [that] [...] makes for a certain unease". ${ }^{194}$ Ironie figuriert für Hutcheons Überlegungen von Anfang an als emotionale Waffe, die ihre Opfer (s. o.) findet und verletzt. ${ }^{195}$

Trotz dieses Fokus auf die Affizierung des ,Opfers ‘ von Ironie liegt für Hutcheon die Antwort auf ihre Frage, warum Ironie überhaupt verwendet werde, doch in der Funktion der Ironie für diejenigen, die sie verwenden. Hutcheon sieht Ironie weder als rhetorische Figur noch - und darin anders als Purdy - als ,extended attitude to life“. ${ }^{196}$ Sie ist stattdessen eine je nach Verwendung oppositionelle, normative oder ambivalente diskursive Praxis oder Strategie, ${ }^{197}$ und sie involviert eine emotionale Ethik. Hutcheon ist interessiert an der

affective , charge" to irony that cannot be separated from its politics of use if it is to account for the range of emotional responses (from anger to delight) and the various degrees of motivation and proximity (from distanced detachment to passionate engagement). Sometimes irony can indeed be interpreted as a withdrawal of affect; sometimes, however, there is a deliberate engaging of emotion $[\ldots] .{ }^{198}$

\subsubsection{Ironie und Politik}

In dem von Hutcheon vernachlässigten Konzept von Ironie als Haltung liegt m. E. gerade die Relevanz der Ironie als Strategie und die Politisierung ihres emotionalen Konnexes begründet. Konzentriert sich Hutcheons Irony's Edge auf die Frage

\footnotetext{
${ }^{192}$ Hutcheon: Irony's Edge, S. 2.

${ }^{193}$ Hutcheon: Irony's Edge, S. 2.

${ }^{194}$ Hutcheon: Irony's Edge, S. 9.

${ }^{195}$ Dieses Unbehagen baut Hutcheon aus: „With irony, you move out of the realm of the true and false and into the realm of the felicitous and infelicitous [...]. Irony removes the security that words mean only what they say. So too does lying, of course, and that is why the ethical as well as the political are never far beneath the surface in discussions of the use of and responses to irony" (Hutcheon: Irony's Edge, S. 14). Dies ist es auch, was Hutcheon mit dem titelgebenden ,irony's edge " meint: „Unlike metaphor or metonymy, irony has an edge; unlike incongruity or juxtaposition, irony can put people on edge, unlike paradox, irony is decidedly edgy" (S. 37).

${ }^{196}$ Hutcheon: Irony's Edge, S. 10.

${ }^{197} \mathrm{Vgl}$. Hutcheon: Irony's Edge, S. 3; siehe auch 29 ff.

${ }^{198}$ Hutcheon: Irony's Edge, S. 15. Hutcheons Fokus scheint dabei jedoch weniger auf der produktiven emotionalen Kraft der Ironie zu liegen als bei der Position der durch Ironie ,Geschädigten", auf die sie immer wieder zurückkommt. Vgl. S. 39ff., wenn sie sich von der affektiven Intention des Ironikers ab- und der Frage zuwendet „how emotion gets talked about and how its edge is seen to cut or abrade from the point of view of the interpreter of irony" (S. 41, Hervorheb. im Original).
} 
nach der Funktion der Strategie, arbeitet sich Colebrooks Irony am Ethos der Ironie ab. ${ }^{199}$ Mehr noch als eine Technik, auf die sie durch die Antikerezeption des Mittelalters und der Renaissance reduziert wurde, sei Ironie bereits in der Figur Sokrates' bei Platon eine Haltung, gar ein Persönlichkeitszug: „Socratic irony is an art or process of self-formation in opposition to fixed meanings, definitions and conventions““. ${ }^{200}$ Ironie ist daher prinzipiell für Colebrook eine ,,mode of self or a way of life". 201

In dieser Konzentration auf die Selbstformierung liegt einerseits die Gefahr der Entpolitisierung, welche im Ironiediskurs um 2001 angemahnt wird. Dabei scheint Ironie aber andererseits per se politisch eingebunden zu sein, egal auf welcher Seite eines alten Disputes über Ironie - rhetorische Figur vs. Haltung - man sie verortet. Colebrook sieht denn in diesem Disput auch eine Auseinandersetzung über den Status von Politik. Betrachte man Ironie als rhetorische Figur, die erkannt werden kann, weil es so etwas wie ein gemeinsames menschliches Verständnis (,understanding') gibt, so Colebrook, so diene sie der Verstärkung von Wahrheit und Konsens. Politik beruht in diesem Modell auf Übereinkunft und Anerkennung, ist Sprache im Sinne eines zu erreichenden Ideals. Wird Ironie jedoch als ,way of life, embodied in the figure of Socrates who refused to represent virtue and the good life as a fixed ideal that could be known", ${ }^{202}$ betrachtet, so wird Ironie als ,the continual questioning or distance from fixed norms" ${ }^{\text {"203 }}$ zur Politik als Praxis, als ,engaged activity achieved through dynamic speech and collective participation“. ${ }^{204}$ Ersteres bezeichnet Colebrook als die Position einer ,politics directed towards community and unity“" ${ }^{205}$ Letzteres als die Position einer Politik als ,rejection, contestation or disruption of shared norms“. 206

In ihrer Zeitdiagnose geht es Colebrook vor dem Hintergrund dieser politischen Grundierung der Ironie um eine ironische Haltung, die durch den Modus kontinuierlichen In-Frage-Stellens Möglichkeiten für andere Perspektiven eröffnet:

\footnotetext{
${ }^{199}$ Dies beginnt schon bei der Rückführung der (mit Hutcheon), duplicity“ sprachspielerischen Hinterfragens auf die erste Verwendung des Wortes eironeia in den Platonischen Dialogen: ,[I]t is through the art of playing with meaning that the interlocutors of a dialogue are compelled to question the fundamental concepts of our language". Colebrook: Irony, S. 6.

${ }^{200}$ Colebrook: Irony, S. 34.

${ }^{201}$ Colebrook: Irony, S. 34.

${ }^{202}$ Colebrook: Irony, S. 44.

${ }^{203}$ Colebrook: Irony, S. 44.

${ }^{204}$ Colebrook: Irony, S. 44 f.

${ }^{205}$ Colebrook: Irony, S. 45.

${ }^{206}$ Colebrook: Irony, S. 45. Diese grundsätzlich politische Note der Ironie thematisiert auch Konstantinou, wenn er die Ironikerin mit Kenneth Burkes Ironieverständnis beschreibt als jemanden, die ,puts herself - or is put - into a relation with her environment, furnishes reasons for actions, and articulates preparartory exercises meant to condition the person for activity“. Konstantinou: Cool Characters, S. $20 \mathrm{f}$.
} 
The ironic subject does not just take part in the discourses and norms that are present; she can ask whose norms these are and whether they are valid. Irony allows for detachment and an ,eternal " point of view; the ironic self can question whether life might not be otherwise, whether, we' might create ourselves differently. Indeed, irony detaches itself from any recognised , we' in order to question and disrupt accepted norms. ${ }^{207}$

Konstantinou übernimmt diese Vorstellung von Ironie als ,,a life, a specific (often oppositional and critical) way of being in and interpreting the world", 208 das politische Bedeutung besitzt:

At the most general level, the ethos of irony is a dialectical model of mental or moral character that invites us to seek disjunctions, contradictions, or mismatches within what we (or our interlocutors) most cherish. And though the attitude of irony does not have any necessary political content - it is not inherently liberal, radical, or conservative - it is inescapably connected to political life because it forms, dissolves, and governs communities and informs action. ${ }^{209}$

In der Formulierung des ,charakterologischen' Zuges der Ironie wird sie so in der Ironieforschung des 21. Jahrhunderts (wieder) mit dekonstruktivem Potenzial aufgeladen. Konstantinou fasst dieses Potenzial und die dazugehörige Praxis wie folgt zusammen:

The ironist has the capacity to unravel power by systematically exposing the infinite web of connotations behind every hegemonic denotation. [...] The ironist debunks rigid ideologies, unweaves foundational ,master narratives' and deconstructs every conceptual or philosophical description of the world through the close investigation of language. To be ironist is to have the power and inclination to reveal the gaps and holes in hegemonic conceptions that pass themselves off as common sense. ${ }^{210}$

Damit unterscheidet sich Ironie in der Postmoderne vom modernen ironischen Subjekt, welches aus einer Position der Distanz heraus spricht. Die Postmoderne ist laut Colebrook und Konstantinou dagegen in Bezug auf Ironie als inhärent widersprüchlich zu betrachten. Denn zum einen lässt sich die Postmoderne als radikale Zurückweisung oder Redefinition von Ironie verstehen:

If irony demands some idea or point of view above language, contexts or received voices, postmodernity acknowledges that all we have are competing contexts and that any implied ,other" position would itself be a context. Postmodernity would be a society of simulation and immanence with no privileged point from which competing voices could be judged. One would have to accept one's own position as one among others, and as thoroughly

\footnotetext{
${ }^{207}$ Colebrook: Irony, S. 122, Hervorheb. im Original.

${ }^{208}$ Konstantinou: Cool Characters, S. xi.

${ }^{209}$ Konstantinou: Cool Characters, S. xi.

${ }^{210}$ Konstantinou: Cool Characters, S. 31.
} 
unoriginal. One would be ironic, not by breaking with contexts but in recognising any voice as an effect of context, and then allowing contexts to generate as much conflict, collision and contradiction as possible, thereby precluding any fixity or meta-position. ${ }^{211}$

Zum anderen lässt sich jedoch auch behaupten, dass sich die Postmoderne allen postironischen Versuchen zum Trotz, Ironie zu überwinden, durch die Unmöglichkeit auszeichnet, Ironie hinter sich zu lassen:

Any attempt to reduce the world to discourses, contexts, language-games or relative points
of view would itself generate a point of view of recognition: the point of view of the post-
modernist who continually affirms the end of meta-narratives, the point of view that is
other than the beliefs of feminism, Marxism, nationalism or any other belief in identity. ${ }^{212}$

Für Colebrook arbeitet postmoderne Ironie mit dieser Widersprüchlichkeit. Es ist gerade der Einsatz von Humor, welcher eigentlich ganz anders arbeitet als Ironie, der dies zeigt. Wie Colebrook schreibt: „In humour, the self appears less as an organised agent or organising subject and more as a collection of incongruous body parts. [...] Humour takes the human subject back or , down' to its corporeal origins“. ${ }^{213}$ Diese Art von Dekonstruktion hin zur Kollektion des Unvereinbaren entspricht nicht nur den Verfahrensweisen vor allem postmoderner Literatur. Sie entspricht auch Sapolskys Vielfalt an Ich-Perspektiven und dem humoristischen Einsatz in A Primate's Memoir, der nicht nur diverse körperliche Funktionen, sondern auch jene intensiven Formen von Emotionalität ins Spiel bringt, die das Subjekt auf seine ,corporeal origins' zu verweisen vermögen.

\subsubsection{Ironische Immunität}

Sapolsky scheint die beiden Fokusse der Ironieforschung - Strategie und Haltung verinnerlicht zu haben: Aus einer ausgewiesen politischen Haltung heraus - der eines , liberals ' - schmiedet Sapolsky unter Einsatz humorisierender Verfahren eine Strategie, die es ihm erlaubt, gleichzeitig in der Auseinandersetzung mit wissenschaftlichen und literarischen Konventionen einen Prozess der Selbstformierung (als Forscher/als Mensch) zu finden, eine emotionale Ethik (in der Forschung) zu diskutieren und sich dabei gegenüber dem Verdacht des Sentimentalismus zu immunisieren.

\subsubsection{Ironie als Kritik}

Die beiden Funktionen von Sapolskys ironischer Strategie, der immunisierende Schutz vor und das Ausüben von Kritik, sind dabei dicht miteinander verknüpft. Sapolskys strategischer Einsatz von Ironie als (Selbst-)Kritik beginnt bspw.

\footnotetext{
${ }^{211}$ Colebrook: Irony, S. 164.

${ }^{212}$ Colebrook: Irony, S. 164 f., Hervorheb. im Original. Vgl. auch Konstantinou: Cool Characters, S. 8 f., $37 \mathrm{f}$.

${ }^{213}$ Colebrook: Irony, S. 137.
} 
bei der Infragestellung von normiertem Textbuchwissen, umfasst typische Züge der Selbst-Demontage, wie sie Hutcheon anführt, und schafft eine historische Kontextualisierung. Dadurch werden sowohl allgemeine Forschungsparadigmen als auch die individuelle Forschungspraxis kritisch beleuchtet. Zugleich wird aber auch das Subjekt der Kritik insofern hinterfragt, als es ein uneinheitliches, ein zumindest durch die unterschiedlichen Ebenen der Erzählung innerlich heterogenes Subjekt ist.

In Bezug auf ,anthropological textbooks“ problematisiert Sapolsky deren „love affair with savanna baboons and their top-ranking male, the alpha male“ (16):

According to the books, the baboons were complex social primates living in open grasslands; they had organized hunts, a hierarchical system, and at their core was the alpha male. He led the troop to food, spearheaded the hunts, defended against predators, kept the females in line, changed lightbulbs, fixed cars, blah blah blah. Just like our human ancestors, the textbooks ached to say, and sometimes even did. Most of that turned out to be wrong, naturally. (16)

Durch die Ergänzung der Aufzählungsreihe mit vermeintlich typischen ,maskulinen“ Tätigkeiten und die Substitution eines ,etc. ' durch das abwertende „blah blah blah" wird die Ideologisierung des Fachwissens im Lehrbuch hervorgekehrt. Zugleich offenbart diese Ironisierung des propagierten wissenschaftlichen Paradigmas Sapolskys eigene Ansicht: „,[N]aturally“, so schließt er die Passage, habe sich das meiste dieser Annahmen als falsch herausgestellt. Er verortet sich selbst also bei einer neueren, fortschrittlicheren Primatologie. ${ }^{214}$

Doch auch mit sich selbst geht Sapolsky ins Gericht, wenn er zunächst schreibt „Salomon was good and wise and just“, dann aber anfügt: „Actually, that's nonsense, but I was an impressionable young transfer male at the time“ (16). Sapolskys Text wimmelt von jenen Formen der ,self-conscious, self-contradictory, self-undermining statement $[\mathrm{s}]^{\text {", }}{ }^{215}$ wie sie Hutcheon als charakteristisch für die ironische Postmoderne betrachtet. Häufig geht es mit Einschüben oder Anfügungen um die Offenlegung eines naiven Anspruchs oder einer idealistischen Vorstellung des damals noch ,unreifen" Forschungsneulings und den Abgleich mit der ,Realität", wie ihn die Perspektive des ,reifen' erzählenden Ichs liefert. So heißt es z. B.:

I had steeled myself for years in expectation and acceptance of the dangers I would face in the bush, had bid farewell to my loved ones at home as if I might not return. I had fully prepared myself for predators and buffalo and poisonous snakes. Instead, a far bigger problem was the constant bugs in the food. (28)

\footnotetext{
${ }^{214}$ Vgl. dazu auch Sanders Pollocks Interpretation von Sapolskys A Primate's Memoir als Positionierung eines männlichen Forschers in der weiblichen Nische der Feldprimatologie in der männlich geprägten Welt der Wissenschaft (,Sapolsky could be considered a man in a woman's world in a man's world“) und in einer sich durch Generationswechsel verändernden Disziplin. Sanders Pollock: Storytelling Apes, S. 189 ff., hier 197.
}

${ }^{215}$ Hutcheon: Politics, S. 1. 
Neben derart unterlaufenen Erwartungen finden sich überspitzte Reaktionen, häufig im inneren Monolog. Als Sapolsky erstmals feststellt, dass säugende Elefantinnen Brüste ausbilden, denkt er zunächst, er halluziniere, weil er vorab die psychischen Begleiterscheinungen der Feldforschung recherchiert hat:

And the natural first reaction is to think, Oh great, I'm such a horny lascivious pathetic adolescent that after a mere month of isolation in the bush I've already cracked, I'm hallucinating breasts the size of Volkswagens on the elephants. Horror, to have one's psychotic break occur so soon, and to have it take the form of puerile sexual obsessions many embarrassing steps below gawking at National Geographic nudies. (28 f., Hervorheb. im Original)

Nicht nur die Naivität des Feldforschers (dem dieses spezielle biologische Detail nicht bekannt war) und die Überzeugung, einem (offenbar erwarteten) psychischen Ausnahmezustand zu erliegen, auch die im Kommentar manifestierte ,double consciousness', das sich zugleich von innen und von außen Betrachten, werden ironisiert.

Meist richtet sich in A Primate's Memoir wie hier die ironisch distanzierende Kritik eines älteren an die Haltung des jüngeren Selbst, wie sie sich besonders günstig in der Reflexion der eigenen Biographie entfalten kann. So beschreibt Sapolsky seine Gründe für eine Reise nach Uganda, kurz nachdem tansanische Truppen den Diktator Idi Amin 1979 gestürzt haben, wie folgt:

I was feeling, atypically for me, a journalistic reflex, a desire to see history being made, and I was moved by this particular history - dancing in the streets, a freed people. [...] [A]nd, intellectually, $[\mathrm{I}]$ thought that it would be important for me to test the ideals of pacifism that I was toying with by observing an undeniably just war. (87)

\title{
Dann fällt sich Sapolsky selbst ins Wort und kommentiert:
}

\begin{abstract}
$\mathrm{Ah}$, this is nonsense, I was twenty-one and wanted an adventure. I wanted to scare the shit out of myself and see amazing things and talk about it afterward. And for the previous month, I had been missing someone badly, and I thought that going to a war would make me feel better about it. I was behaving like a late-adolescent male primate. (87)
\end{abstract}

Sapolskys ironische Haltung zeigt sich dort besonders deutlich, wo er sie gegen sich selbst und sein Selbstkonstrukt in der Vergangenheit wendet und somit für sein Publikum offenlegt, wie wenig verlässlich, stabil oder auch nur aufrichtig die angenommenen Perspektiven, Ideale und Ausdrucksformen sind. Das Subjekt der Kritik, so wird dabei auch deutlich, kann als Instrument der Problematisierung aber nicht als Quelle der Autorität dienen. ${ }^{216}$ Durch ironische (aber nicht

\footnotetext{
${ }^{216}$ Sapolskys ironischer Umgang mit dem autobiographischen Subjekt scheint hier die Subjektivitätsdebatten der postmodernen Autobiographieforschung zu reflektieren und sich eher auf die Seite der dekonstruktivistischen Ansätze zu schlagen, die von einer einschneidenden Trennung des beschriebenen und des schreibenden Selbst ausgehen. Vgl. Marcus, Laura: Auto/biographical Discourses. Theory, Criticism, Practice. Manchester/New York 1994, S. 179 ff., bes. 183, 202 ff.
} 
unbedingt humoristische) historische Vergleiche erzeugt Sapolsky eine Art kritische Position nicht nur zu seinen Haltungen und Idealen, sondern auch zu seinen Handlungen und den Normen, denen diese unterliegen. Etwa wenn er schildert, wie er zu Zeiten der ,Plague', als Rindertuberkulose unter den Pavianen in der Masai Mara um sich greift, Tiere mit Tuberkulin testet, die Infizierten absondert, untersucht, tötet und die Kadaver verbrennt: „There was an analgesic effect to the sheer magnitude of the work, the repetition, the sleep deprivation“" (291), berichtet er zunächst den Effekt dieser Arbeit auf ihn. Dann fährt er fort:

In some ways, that was academic, at least as far as the garbage dump animals [eine andere Paviangruppe] were concerned; whether piecemeal or as a premeditated final solution, we were getting around to killing most of them anyway. The animals passed with exhausting labor, and each day's work ended with a sight that I now recall with nostalgia wasted on long-ago nightmares that eventually end - a massive hole we had dug and the burning of baboon bodies doused with gasoline. (292, Hervorheb. MS)

Diese Formulierungen, die als Anspielungen auf ,Endlösung', Arbeits- und Vernichtungslager gelesen werden können, sind nicht zufällig gesetzt. Als Nachfahre von Opfern der Shoah liegt es für Sapolsky nah, sein Tun historisch zu kontextualisieren: ${ }^{217}$,The repetitive peace of my death camp and crematorium, the calming sadness of smelling the burning“ (292, Hervorheb. MS). Auch an anderer Stelle, wenn es darum geht, dass Sapolsky sich als ,fairly hardened when it comes to the suffering of animals" (219) beschreibt, gesteht er doch bezüglich seiner Laborarbeit (,the suffering that the animals would endure there was appalling“, 219) ein: „I'd have dreams where I was Dr. Mengele“ (220). Sapolsky artikuliert in der Parallelisierung seiner Tierforschung mit der Shoah brachial ein Bewusstsein für problematische Ethiken in der Forschung. Durch die Wahl eines ironischen Erzählmodus, der auf Selbstevaluierungen beruht, erscheint A Primate's Memoir so durchdrungen vom reflektierten Bewusstsein über die eigene Unzulänglichkeit, aber auch von einer kritischen Perspektive auf die Praktiken der Forschung mit Tieren, die deren Tod erfordern.

\subsubsection{Emotion und Ironie}

Sapolskys eigentliches Forschungsthema ist Emotionalität: „What I wanted to study“, erläutert er zu Beginn seiner Memoir, „was stress-related disease and its relationship to behavior“, denn ,your emotional life can affect your health“ (14): ,[T]he main thing I wanted to learn when I first joined the troop was what a baboon's social behavior, his social rank, his emotional life, has to do with the diseases he gets, especially stress-related diseases“ (37, Hervorheb. MS). Wesentlich dabei ist soziale Aggression, die bei den Pavianen hauptverantwortlich für

\footnotetext{
${ }^{217}$ Sapolsky schreibt auf der ersten Seite der Memoir von lang vor seiner Zeit verstorbenen Großvätern, ,[who] were mythically distant enough that I would not be able to pick either out in a picture" ( $P M$, S. 13). Deutlicher wird er zum Ende des Textes, wenn es um die Lamentatio (vgl. Abschn. 3.4) geht: „With the ashes of my ancestors in the death camps“ (S. 301).
} 
Stress ist, und die Sapolsky, wie er später erläutert, aufgrund ihrer Ambiguität als „the most confusing emotion“ (91) erscheint.

Sein eigenes Emotionsleben teilt Sapolskys auffälligerweise qua Darstellungsmodi in zwei unterschiedlich behandelte Bereiche ein: den der intensiven positiven und den der intensiven negativen Affekte. Intensive positive Affekte werden von Sapolsky ausführlich, stark ironisiert und anspielungsreich beschrieben. So kleidet Sapolsky seinen Stolz über seine Fähigkeiten im ,Darting ${ }^{2218}$ der Paviane zu Untersuchungszwecken in ein Mini-Narrativ, das ihn zum Pavianschreck werden lässt:

I am the angel of death. I am the reign of terror, the ten plagues, I am a case of the clap, I am the thing that goes bump in the night. De Shadow [sic], death warmed over. I am the bogeyman with cat eyes waiting until midnight in every kid's closet, I am leering slinky silent quicksilver Baboon terror, I am Beelzebub's bill collector. Another baboon successfully darted. Euphoria. [...] Today I darted Gums, the last baboon on earth I would have ever thought of getting. Wily old bugger, knew my every trick, had eluded me for months. I was near despair at ever getting a blood sample from him, and today, he screwed up. Surrounded himself in presumed safety in a crowd of females, figured he was safe, figured they would take the fall if there was a rubout, figured I'd never dare shoot into a crowd, but he was wrong! They all had their heads turned, he miscalculated the space between two closely spaced trees, and ffft, I sent an anesthetic dart sailing from the blowgun into his ass. Unconscious in four minutes. Musk triumph power loins dawn-of-man science. It was all I could do to keep from savaging his soft underbelly with my canines while he was down. (37, Hervorheb. im Original)

Nicht nur reflektiert Sapolsky hier die Rolle, die er für die Paviane als Schütze aus dem Hinterhalt spielt. Durch eine Reihe von ironischen Anleihen - religiöse (,,angel of death“, „reign of terror“, „the ten plagues“, „Beelzebub's bill collector") und kulturelle (,case of the clap“, „De Shadow“, „the bogeyman“) - sowie ein eigenes Vokabular (,slinky silent quicksilver Baboon terror“) kehrt er auch die Quelle seiner eigenen Emotionalität kritisch hervor, indem er sie im Jadgmotiv verortet: „Euphoria“ entsteht, weil er in der ,[m]usk triumph power loins dawn-of-man science“-Variante einer alten Jagd- und Kampfdramaturgie erfolgreich seine Beute / seinen Gegner erlegt hat. Deswegen der archaische Impuls des ,,savaging his soft underbelly with my canines“, wie er schreibt. Sapolsky reflektiert den ,adrenaline city, androgenic triumph“ (39), der ihn erfüllt, in seiner Funktion: ,[P]erfect to shore up your precarious sense of manhood, and best of all, you're not even doing something appalling like hunting, you're doing it all in the name of science and conservation. You can wipe out innocent beatific baboons and still be a liberal. Oh, joy“ (39, Hervorheb. im Original). Hier zeigt sich der affektkritische Gehalt von Sapolskys Ironie: Indem sie einen analytischen Blick auf die emotionale Selbstformation und ihre so biologischen wie kulturellen

\footnotetext{
${ }^{218}$ Um die Stresshormon-Level der Paviane untersuchen zu können, muss Sapolsky Blutproben nehmen. Dazu betäubt er die Paviane mit Narkotikapfeilen, die er aus einem Blasrohr auf sie schießt. Der Prozess erfordert einiges an Übung mit dem Instrument, vor allem aber sehr viel Umsicht im Umgang mit den getroffenen und dann betäubten Pavianen, die jederzeit Opfer der Aggression anderer Paviane werden können.
} 
Bedingungen richtet, werden Euphorie und Freude seziert bis sie als Ergebnisse einer bestimmten Konstellation aus Forschungsform, Individuum und politischem Ideal darliegen.

Ähnlich wie im obigen Beispiel verfährt Sapolsky auch bei der Schilderung eines Ausfluges in das Dorf zweier seiner kenianischen Mitarbeiter, den er mit seiner Verlobten unternimmt:

\begin{abstract}
Oh, bliss! Euphoria! Lisa and I climbing up an African mountain to reach a farming village, haul up a guitar and recorder, and then spend an evening by the fire, clustered in close with the entire village, teaching them Paul Robeson songs. Ecstasy! Socialist-summer-camp/Eastern-European-folkdancing/free-the-Rosenbergs/Passover-prayer-for-Palestinian-independence/don't-eat-iceberg-lettuce-don't-shave-your-legs-or-armpits heaven!! [...] Oh, more pleasure than I can stand, dip me into agar right now, freeze this moment for the natural history museum, put me in the display case as the euphoric liberal. (249, Hervorheb. im Original)
\end{abstract}

Hier ironisiert Sapolsky den Genuss, den er und Lisa daraus ziehen, in das abgelegene Dorf zu fahren und mit den Einheimischen eine interkulturelle Verbundenheit zu spüren, durch die Einbettung in ein ,Hippie-Narrativ“ der kommun(ard)istisch bewegten Völkerverständigung - wie sich an der langen und diversen Auflistung von derart konnotierten Komposita ablesen lässt. Die Intensität der Empfindung wird in den Ausrufen „Oh, bliss! Euphoria!“” und „Oh, more pleasure than I can stand“ überspitzt und zugleich mit dem Bild erstarrter Beispielund verdächtiger Klischeehaftigkeit verbunden (,dip me into agar“, „freeze this moment for the natural history museum, put me in the display case as the euphoric liberal"). Auch hier ist es dezidiert wieder eine bestimmte politische Haltung - die des sozial bewegten US-amerikanischen Liberalen - die Sapolsky affirmierend einnimmt und von der er sich zugleich ironisierend distanziert. Diese ,duplicity zeigt jene postmoderne, ironische Problematik, die Purdy, Wallace und Hutcheon beklagen: Sapolsky teilt diese liberale Haltung; doch er kann sie nicht ,ernsthaft ${ }^{*}$ beanspruchen, ohne gleichzeitig deutlich werden zu lassen, dass man sie (in der Erzählerposition um 2001) nicht mehr unkritisch - unironisch - teilen kann.

Der zweite Bereich von Sapolskys Emotions(er)leben in A Primate's Memoir betrifft negative Affekte und wird weitaus weniger ironisch oder humoristisch gestaltet. Es ist dabei auffällig, welche Aufmerksamkeit Sapolsky auf einen Emotionscluster rund um Wut, Ärger, Zorn und Aggression legt. Als Stressforscher scheint er der spezifischen emotionalen Reaktion und ihren Ausprägung näher auf den Grund gehen zu wollen. Dazu kann er zunächst aus seinem Erfahrungsschatz als Reisender in Ostafrika schöpfen. Eine Reiseepisode, bei der er von einem Lastwagenfahrer und dessen Freunden gefangen gehalten wird, beinhaltet auch eine körperliche Auseinandersetzung, deren Ablauf und emotionale Details Sapolsky im Präsens wiedergibt: „I begin climbing up again, when Pius wrenches me back, knocks me to the ground. It hurts, I feel a childish rush of tears, of feeling petulant instead of outraged or terrified“ (63). Sapolsky stellt hier die eigene Reaktion - ,kindische Tränen“, ein Gefühl des Trotzes - einer angemesseneren Reaktion - Empörung oder Angst - gegenüber. Eine solche 
Gegenüberstellung von angemessenen und unangemessenen oder abweichenden Emotionen interessiert Sapolsky auch in der Diskussion der Emotionsmuster seiner indigenen Nachbarn, der Masai. Hier ist es zunächst die Gegenüberstellung von Stoischem und Emotionalem: „,[U]sually stoic“, werden Figuren durch einen spezifischen (und wie sich herausstellen soll, die Emotionalität rechtfertigenden) Anlass auf einmal „crying with rage, shivering with emotion and anticipation“ (247). Es geht Sapolsky aber auch um eine Differenzierung von Formen der Wut. Einer seiner einheimischen Mitarbeiter ist ,probably the only person in the bush for a hundred miles in any direction who was angry about Hitler [...] [,] about the role of the Arabs in the slave trade, or the American genocide of Indians, or Israeli treatment of Palestinians“ (73). Dieser Mitarbeiter habe einen „simmering, bitter anger over the injustices“" (73) entwickelt:

Wilson Kipkoi [...] found that everything he learned made his anger grow. He didn't yell, never even spoke loudly, and he wasn't the type that brawled in an explosion of self-depleting anger. His anger simmered and grew, barely fit in his lanky, taut frame, his triangular head. It powered the constant rhythmic throbbing of the vein in the side of his jaw. (74)

Auch Wilsons Vater, Kopf der lokalen Anti-Wilderei-Abteilung der kenianischen Wildschutzbehörde, ist ein Wutmensch, doch ,[h]e had an explosive anger, raged at his men, knocked them over and beat them if they failed to follow his instructions, was loud and abusive and supremely competent“" (75). Verglichen mit seinem Vater, der selbst in seiner Wut kompetent auftritt, ist Wilson für Sapolsky „the antithesis of rage, his anger was ice. He was silent, taut, watchful. He developed traits very rare among bushmen - causticness, irony, cynicism, bitterness. $[\ldots][\mathrm{H}] \mathrm{e}$ used the best weapon that he had evolved in his mind - open contempt" (76). Ironie, ätzender Sarkasmus, Zynismus, Bitterkeit und Verachtung sind hier Folgen einer kalten Wut, eines Ärgers, der kein Ventil kennt.

Dieser Einblick in Wilsons Persönlichkeit ist in A Primate's Memoir ein Vorausblick auf Sapolskys eigenen Wutkomplex: Als es um den Tuberkulose-Ausbruch unter den Pavianen geht, der durch das unethische Verhalten einiger Masai-Mitarbeiter in einer Touristen-Lodge verursacht wurde, die aus politischen Gründen straflos bleiben, sieht sich Sapolsky selbst intensiven Emotionen ausgesetzt: ${ }^{219}$

I indulged myself in a murderous anger at the Masai. (293, Hervorheb. MS)

\footnotetext{
${ }^{219}$ Sapolsky wird auf einige kranke Paviane nahe einer Touristen-Lodge aufmerksam gemacht, die sich als tuberkulös entpuppen. Durch detektivische und forensische Arbeit kann er mithilfe einiger Tiermediziner/innen aus Nairobi ermitteln, dass sich die Erkrankung durch infiziertes Rindfleisch verbreitet hat, das durch eine Art Verschwörung von Koch und Fleischgutachter der Lodge nicht als solches deklariert wurde. Während immer mehr Paviane erkranken und sterben, zieht der Vorfall zu Sapolskys Bestürzung keine Konsequenzen für die (kenianischen) Mitarbeiter oder (britischen) Eigentümer der Lodge nach sich, da Tourismus eine Haupteinnahmequelle für den kenianischen Staat darstellt.
} 
Never in my life have I felt closer to drowning in anger, felt more poisoned, more lost in a corrosive sense of betrayal. I returned [from Nairobi], as requested, withdrew in my fury, $[\ldots]$ I passed each day obsessing over fantasies of vengeance at everyone. [...] I was going to have my revenge. (294, Hervorheb. MS)

\section{[...] [M]y baboons were going to be avenged. (295, Hervorheb. MS)}

Gegen Ende des Memoir stellt Sapolsky sogar reflektierend noch fest: „I continued to spend far too much time obsessing on my revenge" (299, Hervorheb. MS). Er „,walled off the sense of rage that echoes from the time of the plague“ (302, Hervorheb. MS), und kann erst zum Schluss feststellen: „I no longer rage at night with the memoires of that period“" (302, Hervorheb. MS). Der Zorn, den Sapolsky hier empfindet, erwächst aus dem Empfinden eines fundamentalen Unrechts, das den Pavianen, für die er sich als ihr Beobachter verantwortlich fühlt, geschehen ist. Nach aristotelischem Vorbild gesellt sich dieser Wut über ein erfahrenes Unrecht die Rache bzw. Vergeltung als notwendige Folge und Lösung der Affizierung bei. ${ }^{220}$ Sie aus unterschiedlichen, auch forschungspolitischen Gründen nicht ausüben zu können, verlängert die Phase dieser vehementen Wut und ihrer Begleiterscheinungen nicht nur unmäßig, sie führt zur Vergiftung, zu einem zersetzenden Gefühl des Verrats, zu Rachephantasien. Der Zorn wird zu einem pathologischen Phänomen, das Sapolsky nachts wach hält, in Schüben wiederkehrt und das erst Jahrzehnte später abklingen kann. Seinen eigenen Andeutungen bezüglich Wilsons Wutproblematik folgend (,the best weapon that he had evolved in his mind“), lässt sich dieser unmäßige, da unausgelebte Zorn kontrastiv als affektiver Hintergrund für den ironischen Erzählmodus deuten.

Als Gegengewicht zu seinem selbstironischen Umgang mit Freude, Euphorie und anderen starken positiven Emotionen sind gerade die Passagen, in denen sich Sapolsky mit seinem Kernthema, der sozialen Aggression, und mit seinen eigenen negativen Emotionen auseinandersetzt, überwiegend unironisch gestaltet. Der Kontrast betont die ernsthafte Beschäftigung mit diesem Thema. Konfrontiert mit menschlicher Aggression in jenem Ausmaß, wie es nur Krieg liefern kann, reagiert Sapolsky bspw. angesichts eines gewaltvoll zu Tode gekommenen Soldaten wie gefangen, in seiner eigenen Ratlosigkeit angsterstarrt: ${ }^{221}$ „I want to forget this, I want to get the hell out of here, to be home, to be safe. And I stood there, transfixed, unable to move from that spot“ (90); „I stood watching for hours, mesmerised“(91). Sapolskys ernste Faszination für Aggression ist gegründet in der irritierenden Ambiguität

\footnotetext{
${ }^{220} \mathrm{Vgl}$. Aristoteles: Nikomachische Ethik, S. 169.

${ }^{221}$ Wie oben bereits angesprochen, reist Sapolsky 1979 kurz nach dem Sturz des ugandischen Diktators Idi Amin durch tansanische Truppen nach Kampala. Vor seiner Abreise aus dem Kriegsgebiet sucht er die Quelle des Nils auf. Dort, wo der Viktoria-See in den Weißen Nil übergeht, steht ein hydroelektrischer Damm, an dem Sapolsky den Leichnam eines Soldaten entdeckt: ,his hands tied behind his back. A rope had been put around his throat and tied to some piece of machinery inside that hole, such that as the river rose, the man had eventually been swept off his feet, so that he had drowned or choked" (PM, S. 90).
} 
und Reichhaltigkeit dieser ,Emotion“: „Decades later, in the neurobiology classes I teach“, erläutert er im Anschluss, „I always spend some lectures on the physiology of aggression. [...] Somehow, each year, it takes more and more lectures to cover the material“ (91). Dies habe nichts mit einem etwaigen Anwachsen des Wissens zum Thema zu tun:

But somehow, almost embarrassingly, I spend more and more time talking about aggression. I think each year I lecture longer because of that man with his head tied to the dam and because of how long I stood there looking at him, unable to leave. It think it is because of the ambiguity of aggression. It is the most confusing emotion to me, and with the defenses of an academician, I clearly believe that if I lecture at it enough, it will give up and go away quietly, its simultaneous attraction and repulsion will stop being so frightening to me. (91)

Während Sapolsky im Hörsaal Aggression erklärend zu beherrschen versucht, verweist das unironische Schlusskapitel seines Memoir darauf, dass er sich auch im Feld aufgrund der Erfahrungen mit seiner eigenen hilflosen Wut zukünftig vor weiterer, affektiver Verantwortung schützt, indem er sich emotional zurückhält. Er habituiert mit seinem Team eine neue Paviangruppe, jedoch: „I like these animals, but not much more than that, and each year I do less behavioral observation on them and more physiology, in part so that I will not know them well enough to get attached. I am a different person now and at a different point in my life than when I started here" (302).

Trotz dieser an den Beginn von Hopes fiktionaler Erzählung in Brazzaville Beach (vgl. Abschn. 3.5.2) erinnernden Distanzierung von den Tieren und vom Ich seines autobiographischen Bildungsromans endet A Primate's Memoir mit einer Szene des unwissenschaftlichen geselligen Zusammenseins zwischen Mensch und Tier: Sapolsky und seine Frau suchen in ,seiner' Paviantruppe das alt gewordene Männchen Joshua auf, setzen sich neben ihn und essen gemeinsam mit ihm Kekse. Um die Szene nicht allzu sentimental enden zu lassen, greift Sapolsky auf eine korporale Humorisierung zurück und schließt seinen Bericht wie folgt: „He went about it slowly, grasping the end of each [cookie] delicately with his broken old fingers, chewing with small, fussy toothless bites, continuing to fart occasionally. We all sat in the sun, warming ourselves, eating cookies, watching the giraffes and the clouds“ (304, Hervorheb. MS).

\subsubsection{Schutzironie}

Wie sich auch in diesen letzten Sätzen zeigt, ist es die ironische Zuspitzung und humoristische Kommentierung positiven Empfindens - Freude, Begeisterung, Euphorie, Stolz, auch Zuneigung für die Tiere - und die damit implizierte Reflexion der eigenen liberalen Haltung, die Sapolsky im Rahmen seiner Selbstironisierung gegen den Vorwurf immunisiert, mit ihm , gingen die Gefühle durch". Sapolskys ironische Strategie ist hier jener Schutzmechanismus, wie ihn Linda Hutcheon als Grundzug erläutert und Werner Wolf als Schutzironie bezeichnet. Für Hutcheon kann Ironie zum Selbstschutz dienen, indem das ,self-deprecating ‘ 
als Immunisierung (,,a deliberate attempt to render oneself invulnerable“222) fungiert. Wolf geht noch weiter und sieht in der „objektzentrierten Schutzironie“ 223 eine ,positive Ermöglichungsstrategie für problematische Diskurse“. ${ }^{224}$ In Wolfs Modell lässt sich das Verfahren der Selbstironie auf ein Objekt der Ironie übertragen, das dann in ähnlicher Weise gegenüber Angriffen immunisiert wird:

Objektzentrierte protektive Ironie wirkt [...] als auf das Ironieobjekt bezogene Immunisierungs- und Ermöglichungsstrategie: als ein Verfahren, mit Hilfe einer (Schein-)Distanzierung seitens des Ironikers und einer Reduktion von Ernst und ,Größe' des Ironieobjekts dieses gegenüber Angriffen zu immunisieren, um so etwas gegen einen aktuellen, dominanten Kontext zu thematisieren, das in dessen Rahmen inopportun ist. 225

Für Wolf wirkt diese Form der Schutzironie als „Akzeptanzstrategie, als Ermöglichung der Diskursivierung oder Repräsentation eines problematischen Objekts trotz eines ,feindlichen' Kontextes. "226 Genau dies ist es, was Sapolsky in Bezug auf Emotionalität in seinem Text als Strategie betreibt: Er geht auf Distanz zu seinen positiven Affekten, indem er sie mit Ironiesignalen angereichert darstellt; denn sie scheinen nicht mit seinem Bild eines ernsthaften Feldforschers vereinbar zu sein. Durch die Kontextualisierung der Affekte in einem politischen und/oder kulturellen Selbstverständnis, dem sich Sapolsky zurechnet und dem er auch sein Publikum zuzurechnen scheint, sendet er jedoch „Solidarisierungssignale“227 aus, die bereits darauf verweisen, dass die Distanzierung von dieser Emotionalität nur ,scheinbar“ stattfindet. Schließlich ergibt „,das Zusammenwirken von Ironie- und Solidarisierungssignalen ein ambivalentes Resultat“" 228 in dem trotz ,,residualer Distanz “229 die Solidarisierung mit dem Objekt - hier mit Emotionalität - dominiert.

Praktisch wird dadurch anhand des Umgangs mit den intensiven positiven Affekten die Möglichkeit vorbereitet, diejenigen Affekte hervorzukehren, die den Modus der Ironie nicht vertragen. Dies betrifft vor allem Wut, Zorn und Aggression. In der Ernsthaftigkeit, mit der Sapolsky die Affekte des Wutkomplexes beschreibt, bestätigt sich Philip Fishers These von den vehementen Passionen, die anders als ,feelings, the affections or the emotions“ ${ }^{\circ 30}$ „monarchical states

\footnotetext{
${ }^{222}$ Hutcheon: Irony's Edge, S. 50.

${ }^{223}$ Wolf: ,Schutzironie', S. 36.

${ }^{224}$ Wolf: ,Schutzironie', S. 33.

${ }^{225}$ Wolf: ,Schutzironie', S. 34, Hervorheb. im Original.

${ }^{226}$ Wolf: ,Schutzironie', S. 34, Hervorheb. im Original.

${ }^{227}$ Wolf: ,Schutzironie', S. 35.

${ }^{228}$ Wolf: ,Schutzironie', S. 35.

${ }^{229}$ Wolf: ,Schutzironie', S. 35.

${ }^{230}$ Fisher: The Vehement Passions, S. 43. Für Fisher sind Gefühl, Affekt, Sentiment und Passion nicht alternative Bezeichnungen für das gleiche Phänomen, sondern ,language used in service of quite distinct politics of the inner life" (S. 41). Es handele sich bei diesen Begriffen um ein legislatives Vokabular, das ausgehend von einem „template“ (S. 16) als normativem Ausgangspunkt des Denkens über Emotionalität das innere Leben um einen ,Normalfall`von
} 
of being“231 sind und das Individuum so umfassend bestimmen, dass sie sich nicht mit Ironie vertragen. Denn ,[i]rony, and all forms of double consciousness, for which a sense of humor about oneself and one's actions would be the most common case, define by way of contrast the single-mindedness of the passions. The passions are humorless. "232 Emotionen und Gefühle gehen für Fisher Allianzen mit Moderation und Toleranz ein, sie sind modern in dem Sinne, indem sie durch ihre Nähe zur Ambivalenz, zu Ironie und zu „mixed states of many different kinds" ${ }^{\text {"233 }}$ zum Alltag passen. Die vehementen Passionen sind das Außeralltägliche, das Unmoderne, und zwar gerade darin, dass sie Selbsteinheit schaffen. Die Passionen, und diese sind bei Fisher vor allem negativer Prägung (Wut, Scham, Angst, Trauer), vernichten die von Hutcheon identifizierte Relationalität, Inklusivität und Differenzialität der ironischen Haltung, denn ,[t]he vehement passions reinstall an absolute priority of the self, with its claims to be different from and prior to others both in the claims of its will and in its account of the world." 234

Wie der Schock angesichts der Aggression anderer lässt auch die Wut Sapolsky unironisch als Selbst auftauchen. Dieses Selbst verlangt (vergeltende) Gerechtigkeit für das Unrecht an den Pavianen, welches er bezeugen muss. Da diese Gerechtigkeit nicht erlangt werden kann, so lässt sich Sapolskys Emotionsdiskurs verstehen, wird aus der kalten Wut wiederum Ironie, Zynismus, Spott. Darin lässt sich zum einen die Argumentation der Theoretiker/innen der ,Postironie “ entdecken, für die das Leerlaufen des rebellischen Potenzials der ironischen Kritik im Zynismus endet. Zum anderen aber steckt hier ein Hinweis darauf, dass Sapolskys Ironie gerade nicht die von Purdy beklagte ,ironic avoidance of the world, the studied refusal to trust or hope openly“ ${ }^{235}$ sondern ein differenziertes, strategisch zum Einsatz gebrachtes Mittel ist, um ganz in Purdys Sinn für die Übernahme von Verantwortung zu plädieren, ,a renewed idea of public responsibility, the active

\footnotetext{
Emotionalität herum ordne, von dem andere emotionale Phänomene dann mehr oder minder pathologisch abweichen. Während die vehementen Passionen für Fisher Erfahrungen der ungeteilten (vormodernen) Subjektivität sind, bezeichnen die anderen Begriffe eher Phänomene einer (Post-)Moderne, ,[i]n which the self is no longer self-identical [...] no longer characterized by what we might call thoroughness" (S. 43).

${ }^{231}$ Fisher: The Vehement Passions, S. 43.

${ }^{232}$ Fisher: The Vehement Passions, S. 44.

${ }^{233}$ Fisher: The Vehement Passions, S. 46.

${ }^{234}$ Fisher: The Vehement Passions, S. 46. Aus diesem Grund sieht Fisher die ,passions' auch als Scheidefall philosophischer Subjektivitätsentwürfe: „The experience of the unity of the self that takes place within a moment of anger, wonder, grief, or fear - a unity that overrides whatever separation between body and soul we might otherwise take to be the norm - is one primary value of the passions for certain philosophical accounts of the self, and it is equally an embarrassment, on the other side, for other theories of the self" (S. 56).
}

${ }^{235}$ Purdy: For Common Things, S. 185. 
preservation of things that we must hold in common, or, eventually, lose altogether". 236

Sapolskys Ironie ist in jenem Sinn mit Hutcheons ,evaluative edge ' bedacht, wie sie eingesetzt wird, um eine Umwertung zu ermöglichen. Denn diese Ironie dient der retrospektiven, affektiv aufgeladenen (,,affective ,charge “" ${ }^{\star 237}$ ) Bewertung einer Forschungspraxis ebenso wie der Auseinandersetzung mit einer individuellen, biographisch reflektierten Haltung. Sie ist grundsätzlich ein Medium, in dem die Emotionalität des Erzählers Sapolsky thematisiert werden kann: Scheinbar ironisiert abgewertet, kann sie umso deutlicher hervortreten und schließlich unironisch, ,ernsthaft‘ zur Bewertung der dargebotenen Sachverhalte dienen. Gerade durch die Funktionen, die Hutcheon der Ironie bescheinigt, bietet sie sich dem Primatologen Sapolsky strategisch an, um über Emotionalität sprechen zu können: Sie dient der Emphase bestimmter Szenen seines Forschungsberichts, die ihm wichtig sind; sie ist durchweg stilistisches Mittel, seinen Bericht literarisch auszugestalten; sie ermöglicht seinen spielerischen Umgang mit der Retrospektive auf die eigene Forschungsbiographie und den Kulturkontakt. Ironie dient auch der Distanzierung von der eigenen jugendlichen Persona und deren naivem Überschwang; in ihrem dekonstruktiven Potenzial, im kontinuierlichen Unterminieren der eigenen Aussagen, ist sie provisorisch; sie wird ambivalent und oppositional eingesetzt und besitzt Spitzen wie jene gegen die übermächtige Korruption in Kenia, die als Attacken gewertet werden können. Nicht zuletzt dient sie einem Zusammenschluss des primatologischen Autors mit seinen Leser/innen, die als Kompliz/innen in diese Reflektion einer Forschungsbiographie eingebunden werden. Es ist jedoch gerade das ausgeprägt selbstkritisches Potenzial der Ironie als Schutzironie, das Sapolsky befähigt, sowohl von emotionalem Genießen zu schreiben, als auch durch die Diskussion vehementer Affektivität für eine emotional fundierte Verantwortlichkeit für Tier und Forschung zu plädieren, ohne unter einen Sentimentalismus-Verdacht zu fallen.

\subsubsection{Ironische Übertragungen}

Ein derartiges Verfahren beschränkt sich nicht allein auf Emotionalität und wird in seiner Reichweite als Immunisierungsverfahren auch in Sapolskys Umgang mit dem Odium des Anthropomorphismus deutlich. Darin zeigt sich gleichzeitig erneut, wie

\footnotetext{
${ }^{236}$ Purdy: For Common Things, S. 185. Purdys Traktat ist wesentlich ein Aufruf zu mehr politischer Anteilnahme und gemeinsam beanspruchter Verantwortung für die Welt, in der wir leben, für „several sorts of commons, which affect us in different ways“ (S. 186). Auch David Foster Wallace hofft, weniger optimistisch, auf neue Rebell/innen, die wieder bereit sind, etwas zu riskieren (,,risk things. Risk disapproval.“), vor allem eine emotionale Anteilnahme am Geschehen: „The new rebels might be the ones willing to risk the yawn, the rolled eyes, the cool smile, the nudged ribs, the parody of gifted ironists, the ,How banal ${ }^{\circ}$. Accusations of sentimentality, melodrama. Credulity“. Wallace: E Unibus Pluram, S. 193.
}

${ }^{237}$ Hutcheon: Irony's Edge, S. 15. 
eng der Anthropomophismus- in der Primatologie mit dem Sentimentalismus-Verdacht verbunden ist. In A Primate's Memoir finden sich einige Stellen, an denen Sapolsky explizit auf Anthropomorphismus eingeht. So erwähnt er bereits recht früh im Text Debatten in der Tierethologie ,,as to the appropriateness of using emotionally laden human terms to describe animal behaviors“ (24, Hervorheb. MS). Diese emotional aufgeladenen Begriffe entstammen hier dem Bereich der menschlichen Sozialstruktur wie bspw. ,castes', , slaves ' und ,wars'. Sapolsky lehnt deren Verwendung in der Ethologie ab und ordnet sich bei einer wissenschaftlichen Position ein, die in Gegenposition zu Richard Wrangham und Dale Petersons Plädoyer (vgl. Abschn. 3.3.4) behauptet ,that they are very different, and that by saying that all sorts of species take ,slaves', for example, one is subtly saying that it is a natural, widespread phenomenon“" (24). Mit diesem Hinweis auf eine antirelativistische Ablehnung von Anthropomorphisierungen scheint sich Sapolsky also von jeglichen solchermaßen verdächtigen, und vor allem emotional aufgeladenen Verfahrensweisen in der Beschreibung und Interpretation tierlichen Verhaltens abgrenzen zu wollen. Und doch wimmelt Sapolskys Text von Anthropomorphismen und emotional aufgeladenen Beschreibungen ,seiner' Tiere. Er muss deren Gebrauch an eben jener Textstelle auch eingestehen: „Nevertheless, Solomon did something that day that I think merits the emotion-laden term that is typically used to describe a human pathology. Solomon chased Devorah, seized her near an acacia tree, and raped her" (24, Hervorheb. MS).

In einem Interview in The Atlantic anlässlich der Veröffentlichung von A Primate's Memoir bezieht Sapolsky erneut Position gegen anthropomorphisierende Verfahren:

I'm completely opposed to anthropomorphism - it's lousy science. That's why I'm not doing anything anthropomorphic in my writing about the baboons. If it seems like there's something relating to human attributes in there, it's because they're close relatives. I am not ascribing anthropomorphic, or hominid-like traits to them, but I sure am ascribing complex social primate traits to them, and they seem familiar because they are familiar they're our close relatives in lots of ways. ${ }^{238}$

Sapolsky scheint hier differenzieren zu wollen zwischen Anthropomorphisierungen (,lousy science“) und wiedererkennbaren, komplexen sozialen Zügen der Primaten, die nur menschlich wirken, weil Menschen eben auch Primaten sind. Dieser Differenzierung widerspricht die Stilisierung von A Primate's Memoir deutlich. Weil Sapolsky nicht darauf verzichten will, , seine ' Paviane als Personal einer hochspannenden ,Soap Opera“ darzustellen, ist Rebecca z. B. ,the prebuscent daughter of beautiful, bound-for tragedy Bathsheeba. She lacked the classic looks of her mother but had a fresh baboon-next-door quality to her" (96, Hervorheb. MS). Sofort wird diese Beschreibung als ,appalling anthropomorphism“ (96) ausgewiesen, aber weiter verstärkt, indem Sapolsky berichtet, dass er der Pavianin auch noch „,ear tags“" verliehen habe, ,that stuck out like barrettes for her nonexistent pigtails“ (97). Sapolsky schafft sich durch die öffentliche

${ }^{238}$ Bacon: Monkeys, o. S. 
Distanzierung von Anthropomorphismus in der Forschung und die ironisch-kritische Kommentierung von Anthropomorphismen im Text die Möglichkeit, unter dem Deckmantel einer ,parody of anthropomorphizing“ ${ }^{\text {‘239 }}$ weiterhin seine Tiere geradezu lustvoll anthropomophisierend und emotionalisierend $\mathrm{zu}$ betrachten - versehen mit der Schutzbehauptung ,this sort of thing is meant to be tongue in cheek“. ${ }^{240}$

,Tongue in cheek' hat Sapolsky die Lizenz gerade über jene Themen zu schreiben, die nicht nur aus wissenschaftlichen Texten in autobiographische Reflexionen verbannt, sondern auch in diesen zum Problemfall für die Rolle als wissenschaftliche Forscher/innen-Figur werden können, von deren Formierung die Forschungsmemoiren im Regelfall berichten. Als Text mit literarischem Anspruch kann Sapolskys A Primate's Memoir deutlicher als andere hier diskutierte Texte auch auf literarische Strategien zurückgreifen, um von seiner Forschungsarbeit und Selbstformierung als Primatenforscher zu erzählen. Zugleich aber lässt sich die Form der Verhandlung von Affekten im Feld in A Primate's Memoir vor allem vor dem Hintergrund einer effektiven Strategie der immunisierenden Ironie verstehen, die in den dem Text zeitgenössischen Diskurs um (Post-)Ironie eingebettet scheint und sich aus dem in diesem Diskurs zutage geförderte Spektrum an Funktionen von Ironie - rhetorische Strategie, Ethos, Kritik, Schutz - speist.

\subsection{Von deutscher Art und Aff? Forschungsmemoiren im 21. Jahrhundert}

Wie sich Alles in der Welt ändert: so mußte sich auch die Natur ändern, die eigentlich das griechische Drama schuf. [...] Man konnte zwar das Uralte, oder gar von andern Nationen ein Fremdes herbei holen, und nach der gegebnen Manier bekleiden: das that Alles aber nicht die Würkung: folglich war in Allem auch nicht die Seele: folglich wars auch nicht (was sollen wir mit Worten spielen?) das Ding mehr. Puppe, Nachbild, Affe, Statüe, in der nur noch der andächtigste Kopf den Dämon finden konnte, der die Statüe belebte. ${ }^{241}$

Im Vergleich zu Robert Sapolskys Forschungsmemoiren hat die deutschsprachige Primatologie ein Problem, zumindest dort, wo sie sich in den ersten beiden Dekaden des 21. Jahrhunderts dieser Gattung widmet: Volker Sommers Schimpansenland ${ }^{24}$ von 2008 will ,persönliche Saga“, ,grüne Kampfschrift und Zeugnis des faszinierenden Abenteuers Wissenschaft" (9) sein und liefert profundes Wissen über

\footnotetext{
${ }^{239}$ Bacon: Monkeys, o. S.

${ }^{240}$ Bacon: Monkeys, o. S.

${ }^{241}$ Herder, Johann Gottfried: Von deutscher Art und Kunst. In: Ders.: Schriften zur Ästhetik und Literatur 1767-1781. Hg. von Gunter E. Grimm. Frankfurt a. M. 1993 (Werke in zehn Bänden, Bd. 2), S. 443-562, hier S. 503.

${ }^{242}$ Sommer, Volker: Schimpansenland. Wildes Leben in Afrika. München 2008. Im Folgenden, wenn zur Unterscheidung nötig, als $S P$ geführt; Seitenangaben im Text.
} 
Schimpansen und Details der Forschungspraxis. Es steht aber in der Tradition des Afrikareiseberichts und seines zumindest problematischen Umgangs mit der einheimischen Bevölkerung und der deutschen Kolonialkultur. ${ }^{243}$ Julia Fischers 2012 erschienenes Affengesellschaft ${ }^{244}$ liefert einen umfassenden und informativen Überblick über den primatologischen Forschungsstand, hangelt sich jedoch jenseits des Wissensreferats gerade in den Anleihen aus dem Genre der Forschungsmemoiren von Phraseologismus zu umgangssprachlichem Ausdruck, besonders dort, wo die Erzählerin als fühlendes Subjekt in Erscheinung tritt. Dies erstaunt zumindest insofern, als der deutsche ,Gründervater' der Ethologie bzw. Tierpsychologie, Konrad Lorenz, zur Rehabilitierung seines durch eilfertige Zusammenarbeit mit den Nationalsozialisten lädierten wissenschaftlichen Rufs bereits 1949 ,Tiergeschichten' veröffentlichte, um in einem zunehmend populär werdenden Format von seiner Tierforschung zu berichten. ${ }^{245}$ Auch mit Bernhard Grzimek bietet sich ein deutscher Tierforscher als populärer Autor zum literarischen Vorbild an. ${ }^{246}$ Sowohl Sommer als auch Fischer sind zudem gut mit der englischsprachigen Forschung und der Arbeit vieler der auch in der vorliegenden Arbeit behandelten Autor/ innen vertraut. ${ }^{247}$ An Genre- und Stil-Vorbildern für die eigene populärwissenschaftliche Veröffentlichung fehlt es also nicht. Schimpansenland ist zudem nur die letzte einer Reihe populärwissenschaftlicher und literarischer Publikationen von Volker Sommer. ${ }^{248}$

\footnotetext{
${ }^{243}$ Wolfgang Struck spricht von der ,kulturellen Virulenz des deutschen Kolonialismus“ trotz seiner vergleichsweise kurzen Dauer. Zu den „,erfolgreichsten Spielarten“ einer solchen kolonialen Kultur gehört neben dem Kolonialroman vor allem der Reisebericht. Struck, Wolfgang: Die Eroberung der Phantasie. Kolonialismus, Literatur und Film zwischen deutschem Kaiserreich und Weimarer Republik. Göttingen 2010, S. 13. Honold/Simons stellen gar die Frage, ob man vor dem Hintergrund des ausgeprägten „,kulturellen Resonanzraum[s] kolonialer Bestrebungen einschließlich ihrer prä- bzw. postkolonialen Ausläufer" nicht von Kolonialismus als Kultur sprechen müsse. Honold, Alexander/Simons, Oliver: Einleitung: Kolonialismus als Kultur? In: Dies. (Hg.): Kolonialismus als Kultur. Literatur, Medien, Wissenschaft in der deutschen Gründerzeit des Fremden. Tübingen/Basel 2002, S. 7-15, hier S. 9.

${ }^{244}$ Fischer, Julia: Affengesellschaft. Berlin 2012. Im Folgenden, wenn zur Unterscheidung nötig, als $A G$ geführt; Seitenangaben im Text.

${ }^{245}$ Siehe Lorenz, Konrad: Er redete mit dem Vieh, den Vögeln und den Fischen. Wien 1949. Zu Lorenz' Mitarbeit an der nationalsozialistischen Biologie und der Rehabilitation auch durch das Medium des populären Tierbuches vgl. Föger, Benedikt/Taschwer, Klaus: Die andere Seite des Spiegels. Konrad Lorenz und der Nationalsozialismus. Wien 2001, S. 78 ff., 132 ff.; Burkhardt, Richard W.: Patterns of Behavior. Konrad Lorenz, Niko Tinbergen, and the Founding of Ethology. Chicago/London 2005, S. $231 \mathrm{ff}$.

${ }^{246}$ Volker Sommers Forschungsmemoiren adaptieren sogar einen Titel von Grzimek: Grzimek, Bernhard: Flug ins Schimpansenland. Reise durch ein Stück Afrika von heute. Stuttgart 1952.

${ }^{247}$ Volker Sommer hat den Lehrstuhl für Evolutionäre Anthropologie am University College London inne. Julia Fischer, mittlerweile Professorin für Kognitive Ethologie an der Universität Göttingen und Leiterin der gleichnamigen Forschungsgruppe am Deutschen Primatenzentrum, kann auf mehrere Forschungsaufenthalte in den USA, unter anderem an der Harvard University und als Postdoktorandin bei Dorothy Cheney und Robert Seyfarth an der University of Pennsylvania, zurückblicken.

${ }^{248}$ Die Publikationen Sommers umfassen sowohl Sachbücher und weitere Forschungsmemoiren als auch Essays, Erzählungen und ,Poetische Annäherungen', vgl. Volker Sommer, University College London, http://www.ucl.ac.uk/anthropology/people/academic-teaching-staff/volker-sommer/\#books (22.05.2019).
} 
Gleichzeitig mit dem vergleichsweise saloppen Stil und der Wahl eines traditionsreichen Genres fällt an den beiden Texten etwas auf, das sie von den meisten der hier behandelten Forschungsmemoiren und Feldberichte abhebt: der Mangel an emotionalen Auskünften aus ,dem Feld' und das Fehlen individuierter Affen. Sommer fährt zwar ausdrücklich nach „Afrika“ (Nigeria), um das Abenteuer zu suchen - Schimpansenland wird sogar auf dem Buchumschlag beworben mit „In Afrika sind noch Abenteuer zu erleben“ -; sein Bericht verrät aber kaum große Affizierungen in der Feldforschung. Julia Fischer ist in Affengesellschaft touristisch beeindruckt von der Landschaft und Abgeschiedenheit eines Forschungscamps, die Tiere scheinen sie jedoch größtenteils ,kalt zu lassen“. Im Folgenden soll nun nach den Hintergründen dieser Befunde gefragt werden: Wie und warum sind diese Texte stilistisch auffallend salopp, umgangssprachlich geprägt oder einer problematischen Tradition des Reisetextes verschrieben? Weshalb spielen die Affekte der Forschenden als Forschende in diesen beiden jüngsten, deutschsprachigen Veröffentlichungen eine so geringe Rolle? Lässt sich ein Zusammenhang herstellen zwischen Stil, porträtierter Forschungsform bzw. -methode und Affektregime? Welche Art von Emotionalität wird schließlich in den Texten doch thematisiert und in welchen Kontext lässt sich diese stellen? Was kann dies letztlich über die Rolle von Affekten in der Primatologie des 21. Jahrhunderts aussagen? Die leitende These lautet hierbei, dass die methodische und strukturelle Ausprägung der praktischen primatologischen Forschung im 21. Jahrhundert den Stil des Erzählens von der Forschung und den Affekten vorgibt. Sowohl Fischers als auch Sommers Text geben Hinweise darauf, wie sich die Freilandforschung mit Affen seit den Anfängen bei Schaller, Goodall, Fossey, Galdikas, Strum etc. verändert hat und wie dies das Erzählen von der Forschung und von der eigenen Affektivität beeinflusst.

Die beide Texte sollen hier jeweils für sich hinsichtlich des Stils des Textes, des Zusammenhangs von Erzählen und der Praxis der darin beschriebenen Feldforschung und der Darstellung der Affekte der Forschenden untersucht werden. Denn sie sind unterschiedlich gestaltet: Fischer stellt mit Affengesellschaft vordergründig nicht den Anspruch, einen Feldforschungsbericht zu liefern, sondern zieht Hinweise auf eigene Forschungsaufenthalte nur heran, um in ein neues Thema einzuführen oder Thesen zu illustrieren. Ihr Ziel ist, die wissenschaftliche und populäre Faszination für Affen zu begründen und ihr Forschungsinteresse darzustellen. „[V]on den Reizen, Herausforderungen und absonderlichen Begebenheiten [zu] erzählen, die sich einem bei der Freilandforschung in tropischen Ländern bieten“ (10), ist ihr eher eine Zugabe, ,weil so viele danach fragen, wie das Leben ,in Affengesellschaft" so ist" (10). Nichtdestotrotz wird durch jene Passagen ihre Formierung als nunmehr etablierte Affenforscherin dargestellt, so wie es das Genre der Forschungsmemoiren leistet. Sommer dagegen formt Schimpansenland explizit und durch allerlei Anklänge zu einem Reisebericht „,aus dem Dschungel“", „,der Wildnis“, „Wald und Wipfel“ oder dem „Märchenwald“ (so verschiedene Kapiteltitel), der durch die Erzählung von der Gründung und dem Ausbau einer Forschungsstation deutlicher als primatologische Forschungsmemoiren angelegt ist, zugleich aber auch viel stärker als die englischsprachigen Beispiele (und als Julia Fischer) Traditionen des Afrikareiseberichts aufnimmt. 


\subsubsection{Von den Erfahrungen anderer erzählen: Julia Fischers Affengesellschaft (2012)}

\subsubsection{Stil: Sprachklischee und Nahbarkeit}

Julia Fischers Forschungsinteresse, so informiert sie ihre Leser/innen im ,Pro$\log ^{\prime}$ von Affengesellschaft, gilt der Frage, ,in welchem Verhältnis Sozialsystem, Intelligenz und Kommunikation der Affen zueinander stehen“, bzw. wie sie es verdichtet: „Wie schlau sind Affen eigentlich?“ (10). Diese Art salopper Zuspitzung zieht sich durch Fischers Text. Wenn es um das Altern von Berberaffen geht, sind die Männchen „,meist noch ganz gut in Form“ (27); die „Verhältnisse [sind] oft komplexer als die graue Theorie“ (28); Fäkalien sind „Affenscheiße“ (40) oder „Gold der Primatenforscher“ (40); Affen haben „einen Jieper auf Feigen“ (112) und bekommen „eine glatte Eins“ (145). Vieles wird „absolut köstlich“ (142) gefunden und Fischers Text ist bevölkert von Modifikatoren (bspw. „bizarr“, 20; „enorm“, 59; „,extrem[]“, 31). Es finden sich Phraseologismen wie „Vorsicht ist die Mutter der Porzellankiste“ (185), „hüten wie unsere Augäpfel“ (197), „,nicht ganz koscher“ (209), oder „Geschenk des Himmels“ (48). Fischer selbst ist im Text „,mucksmäuschenstill“ (210), ,wie elektrisiert“ (219), hat „,[n]ach anderthalb Jahren und viel But, Schweiß und Tränen“ ihre Experimente „im Kasten“ (215), ist „heilfroh“ (208) oder ,wie vom Donner gerührt“ (47). Auch in Bezug auf die eigene Forschungsbiographie verwendet Fischer Redensarten: Die Primatologie war ihr „sicherlich nicht in die Wiege gelegt“ (11), sie macht ihre ,ersten Gehversuche“ (34) als Forscherin und ist „frischgebackene Doktorin“ (47).

Dieser saloppe Stil samt seiner Sprachklischees steht im Gegensatz zu Passagen, die dem Argumentationsstil wissenschaftlicher Arbeiten folgen: „Im Folgenden werde ich mich zunächst [...]. Danach werde ich mich der Frage zuwenden [...]. Dabei werde ich zunächst danach fragen [...]. Anschließend behandele ich [...]“ (97). Es lässt sich vermuten, dass das Sprachklischee der Autorin somit nicht einfach unterläuft. ${ }^{249} \mathrm{Im}$ Sinne Barbara Sandigs ist ,Stil“ zu verstehen als ,,variierende Sprachverwendungen und Textgestaltungen, denen relativ zu bestimmten Verwendungszwecken und Verwendungssituationen von den Beteiligten bestimmte sozial und kommunikativ relevante Bedeutungen zugeschrieben“" und

\footnotetext{
${ }^{249}$ So lautet der Verdacht bei Villiger: „Von einem ,Sprachklischee“ können wir dann sprechen, wenn jemand vermutlich den guten Willen hat, einen persönlich erfahrenen Sachverhalt kundzutun, aber daran scheitert, daß ihm sein Sprachgedächtnis in die Quere kommt und ihm für das, was er sagen will, eine vorfabrizierte Wendung zur Verfügung stellt. Jeder von uns verfügt nämlich über einen erheblichen Vorrat fester Prägungen, Sprachklischees, die sich immer wieder zwischen seine Erlebnisse - seien es Sinneserfahrungen, Gefühle oder Gedanken - und ihre Wiedergabe in eigenen Worten drängen. Viele dieser Klischees sind durchaus unentbehrlich“. Villiger, Hermann: Gutes Deutsch. Grammatik und Stilistik der deutschen Gegenwartssprache. Frauenfeld 1970, S. 211 f. Burger et al. sprechen im Handbuch der Phraseologie vom Klischee auch als Filter bei der Verbalisierung, vgl. Burger, Harald/Buhofer, Annelies/Sialm, Ambros: Handbuch der Phraseologie. Berlin/New York 1982, S. 18.
} 
die „prinzipiell frei gewählt werden“ ${ }^{250}$ Der saloppe Stil und die klischierte Ausdrucksweise für Eindrücke und Empfindungen schaffen in ihrer umgangssprachlichen Eingängigkeit vertrauliche Nähe zwischen der Autorin, Erzählerin und (überwiegend) Protagonistin Fischer und ihrem Publikum und vor allem eine einfache Vermittelbarkeit von Wissen: Die komplexen Wissensbestände über Affen und Affenforschung, die in Affengesellschaft ausgebreitet werden, treffen auf einen möglichst allgemein vertrauten Sprachgebrauch. Keinesfalls soll wohl der Eindruck aufkommen, hier handele es sich nur um jene ,graue Theorie“ (28) oder gar ,Intelligenzlertum‘.

\subsubsection{Praxis: Anekdotisches Erzählen vs. kollektive Forschung}

Auch der Einsatz von illustrierenden Passagen über Affen verweist auf eine Textstrategie, die Wissen eindrücklich machen soll. Obwohl es heißt, ,,[d]ie Protagonisten dieses Buches sind Berberaffen, Bärenpaviane und Guineapaviane“ (15), kann nicht die Rede davon sein, dass Tiere tatsächlich in der Rolle von Protagonist/innen das Erzählen von der Forschung strukturierten, wie sich dies beispielsweise bei Goodalls schimpansischem oder Strums und Sapolskys pavianischem Personal behaupten ließ. Beobachtungen zu einzelnen Affen werden nur als Einstieg in theoretische oder erläuternde Ausführungen genutzt. So wenn Fischer zu Beginn des zweiten Teils des Buches („,Kognition“, 87 ff.) mit einem Absatz über das Bärenpavianweibchen Amelia beginnt, welches ein anderes Weibchen, Palm, beißt (89). Die beiden Weibchen werden jedoch nur in dieser expositorischen Funktion angeführt und verschwinden danach aus dem Text. Obwohl zu Fischers Forschungsinteressen auch das Altern von Affen gehört, spielt die Dokumentation von primatischen Lebensgeschichten, jene in der vorliegenden Studie bereits mehrfach angeführten ,primate life stories', wie sie die ethologischen Forschungsmemoiren sonst bevölkern, keine Rolle. Sie verweist zwar darauf, dass es auch einzelne Affen gibt, die sie „besonders gern mag“ (167), lässt diese jedoch nicht als Individuen oder gar als Persönlichkeiten in Erscheinung treten.

Die Felderfahrung wird über Fischers Schilderungen ihrer Forscherinnenbiographie und über diese wenigen illustrativen Affenbeispiele angeführt, jedoch nicht konsequent chronologisch und narrativ angeordnet. Die Feldforschung besteht aus einem Aufenthalt im Affenpark im französischen Rocamadour bzw. in Marokko (42 f.), einem eineinhalbjährigen Aufenthalt in Botswana im von Dorothy Cheney und Robert Seyfarth etablierten ,Baboon Camp' (47 f., 51 ff.), einer „Expedition in den Senegal“ (67 ff.), die der Gründung eines Schimpansenforschungscamps vor Ort vorausgeht, und einem kürzeren Ausflug auf die karibische Insel St. Kitts, wo die Rufe von Grünen Meerkatzen aufgenommen werden. Die Erwähnung dieser Feldforschungserfahrung ist instrumentell: Fischer führt die Aufenthalte und Expeditionen an, um primatologische Forschungsthemen oder ihre Forschungsbiographie zu unterfüttern. Dabei gerät sie mit den narrativen

${ }^{250}$ Sandig, Barbara: Textstilistik des Deutschen. Berlin/New York ${ }^{22006, ~ S . ~} 2$. 
Konventionen der Forschungsmemoiren in Konflikt, indem sie zwar die Form der Anekdote wählt, diese aber kaum ausgestaltet. Die folgenden Beispiele können dies illustrieren.

In Frankreich stellt sich Fischer und ihrem Forschungspartner Kurt Hammerschmidt die Frage, ob der Bärenpaviannachwuchs nur im sicheren Freizeitgehege von Rocamadour in der Dämmerung schreit und herumspringt oder ob er dies auch in weitgehend freier Wildbahn tut. Sie beschließen daher, Bärenpaviane in Marokko zu beobachten:

Wir kauften einen alten VW-Bus, die ,Else', mit der wir bergab und mit Rückenwind auf 90 Stundenkilometer kamen, und traten mit ihr die weite Fahrt nach Algericas im Süden Spaniens an, um von dort nach Marokko überzusetzen. Es war März, und im mittleren Atlas lag noch Schnee, als wir in der Gegend ankamen, in der schon die Pioniere der Berberaffenforschung, John Deag und David Crook, ihre Beobachtungen gemacht hatten. Bereits am ersten Abend hörten wir aus den Kronen einer mächtigen Zeder das uns so vertraute Geschrei der kleinen Affen. (43)

Mit dieser kurzen Passage, die nostalgisch das Fahrzeug und die Wetterlage betont, ist der Ausflug nach Marokko für Fischer vollständig abgebildet. Eine Pointe bleibt aus.

Bei der Schilderung der sie andeutungsweise prägenden Zeit der Pavianbeobachtung in Botswana überwiegt die Erinnerung an das romantische Setting des Forschungscamps das Interesse an den Tieren vor Ort: Das Okavango-Delta, in dem das ,Baboon Camp“ angesiedelt ist, liegt „weitab von jeglicher Zivilisation“, „mitten in der Wildnis“ (51). Für Fischer „,bleibt dieser Ort der schönste auf der ganzen Welt" (51). Im Vordergrund stehen hier die Annehmlichkeiten des Camps (fließendes Wasser, Solaranlage, Kühlschrank) und das ,romantische Flair`:

In den Abendstunden saßen wir auf der Terrasse vor der Küchenhütte und genossen den spektakulären Blick auf die Landschaft. Das wirklich Außergewöhnliche aber war die exquisite Einsamkeit: Wenn die Feldassistenten am frühen Nachmittag gegangen waren, waren wir nur noch zu zweit - abgesehen von den vielen wilden Tieren. (52)

Dies klingt wie eine Überleitung zu einer bemerkenswerten Erfahrung mit diesen ,wilden Tieren“; eine solche bleibt jedoch aus. Es heißt im nächsten Satz nur noch, das Camp sei mittlerweile aufgegeben worden („Der Zauber ist für immer vorbei“, 52). Vom Forschungsalltag erfährt man wenig, von einzelnen tierlichen Individuen nichts. Stattdessen wird aber ein Überblick über die an diesem Ort abgehandelten Forschungsthemen und die Geschichte des Forschungsortes geboten.

Auch die Reise in den Senegal, die Fischer wieder mit Kurt Hammerschmidt unternimmt, um dort die Möglichkeiten für eine neue Feldstation zu überprüfen, wird lediglich hinsichtlich touristischer Charakteristika ausgestaltet:

In Dakar angekommen, quartieren wir uns erst mal in dem uns empfohlenen Etablissement La Brazzerade ein, das sich etwas außerhalb der Stadt im Bezirk Ngor befindet. Es ist sehr schön am Strand gelegen. Eine kleine Schaluppe fährt zur gegenüberliegenden Île de Ngor. Im Brazzerade gibt es ausgezeichnetes Essen, vor allem frischen 
Fisch vom Grill, und ein sehr gemischtes Publikum, das von der senegalesischen Fußballnationalmannschaft bis zu französischen Großwildjägern und lokalen Geschäftsgrößen reicht. Aufreizend angezogene senegalesische Damen warten auf Kundschaft, dazwischen tummeln sich ein paar Rucksacktouristen, und auch Vertreter der örtlichen Expatries sind hier anzutreffen. Ich gehe jedes Jahr dorthin, wenigstens zum Essen, und freue mich immer, die Belegschaft wiederzusehen. (67, Hervorheb. im Original)

Als Erstes fuhren wir zum Hotel Simenti, eine in Beton gegossene Scheußlichkeit aus den 1960er Jahren, die einst als Großwildjägerlodge gedient hatte. Großartig allerdings ist die Lage: Von der Terrasse des Hotels blicken die Gäste auf eine Biegung des Gambia-Flusses und können die Krokodile und Flußpferde am Ufer beobachten. (69)

Der Reiseführerstil solcher Beschreibung setzt sich bis hin in die Schilderung des später entstehenden Camps selbst fort: In diesem, so preist Fischer an, lebt man „mit den Wildhütern Tür an Tür“ und erlebt ,in ganz anderem Ausmaß die Chancen und Risiken interkultureller Kommunikation“ (70). Es gibt zweimal pro Tag „warmes Essen, das meist sehr gut ist“ (70).

Ein kürzerer Ausflug nach St. Kitts, ,ein[] eher bizarre[s] Kapitel[] in meiner Geschichte als Affenforscherin“ (205), wo die Postdoktorandin Fischer mit einer Kollegin zusammen im Auftrag des US-amerikanischen National Institute of Health Lautaufnahmen von Grünen Meerkatzen erstellen soll, wird ebenfalls als (scheiterndes) touristisches Reiseabenteuer aufbereitet: „Zehn Tage Karibik, alles bezahlt [...]. Einige Wochen später saßen wir im Flugzeug nach St. Kitts, einer Insel, die durch eine vulkanische Gebirgskette geprägt ist und einen Haufen verlorener Seelen beherbergt" (206). Die Verhältnisse sind jedoch alles andere als das erhoffte Karibik-Idyll - die Insel ist teuer, schmutzig, voller Drogenkriminalität -, so sind die beiden Primatologinnen ,heilfroh, als wir endlich wieder im kleinen Propellerflugzeug Richtung Miami saßen“ (208). Über die Forschungszeit selbst hat man auch hier wieder nicht viel erfahren. Die Details dieser Reise werden antiklimaktisch verdichtet:

Von mehreren Männchen konnten wir Alarmrufe aufnehmen. Marta hatte sich ein Tuch mit Leopardenmuster umgehängt und sich auf allen vieren an die Affen herangeschlichen. Die Rufe der Affen klangen genauso wie die ,Leoparden-Alarmrufe‘ der Grünen Meerkatzen in Kenia [...]. (207)

Fischer zieht das Anekdotische als „Erzählung von einem merkwürdigen Vorfall“, ${ }^{251}$ wie sie das Reallexikon definiert, heran, um ganz im rhetorisch intendierten Sinn ,zur Auflockerung des Ernstes, zur Veranschaulichung eines Charakters, zur Befriedigung der Neugierde“" 252 beizutragen, verwendet die Form aber nicht in der Art, wie sie beispielsweise Sapolsky durchweg nutzt, um autobiographische Geschichtsschreibung zu betreiben. Stattdessen unterläuft der Text kontinuierlich

\footnotetext{
${ }^{251}$ Schlaffer, Heinz: Ankedote. In: Harald Fricke/Klaus Weimar/Jan-Dirk Müller (Hg.): Reallexikon der deutschen Literaturwissenschaft. Bd. 1, 3. Berlin/New York 1997, S. 87-89, hier S. 87.

${ }^{252}$ Schlaffer: Anekdote, S. 87.
} 
Erwartungen bei seinen Lesenden. Dies vollzieht sich nicht nur auf der formalen, sondern auch auf der inhaltlichen Ebene. Denn auch dort, wo es bei Fischer in der Feldforschung explizit um die „Herausforderungen praktischer Art“ (81) geht, handelt es sich um Erläuterungen der bürokratischen Hürden vor Ort, „das Überleben im bürokratischen Dschungel“" (81), nicht um Schilderungen von individuellen Forschungserfahrungen, Gefühlslagen oder gar emotionalen Ausnahmesituationen, wie sie den Bericht vom Leben und Arbeiten, in Affengesellschaft bei den anderen hier untersuchten Primatolog/innen bevölkern, und wie sie Fischer in ihrem Prolog andeutet. Es gibt nur eine einzige Passage, in der Fischer auf ihr affektives Erleben im Feld im Umgang mit Affen eingeht. In dieser geht es um das Gelingen von sogenannten Playback-Experimenten:253

\begin{abstract}
Nach vielen Stunden ergibt sich dann endlich eine Gelegenheit - ich schicke meinen Assistenten los, den Lautsprecher zu verstecken und alles in Position zu bringen, schalte die Videokamera an, sage mein Sprüchlein auf [...] und gebe dem Assistenten das Zeichen, den Laut abzuspielen. Mein Puls steigt, die Kamera wackelt ein wenig ... und nichts passiert. Das Tier steht auf und trollt sich. Kalter Schweiß tritt mir auf die Stirn - was ist nun wieder schiefgegangen. [...] Und während meiner Dissertation stand ich manches Mal am Abgrund der Schlucht, die sich am Rande des Affenparks entlangzieht, und war kurz davor, die gesamte Ausrüstung dort runterzuschmeißen. Ich vermutete irgendwann, dass die Affen ein Experiment mit mir durchführten, um meine Belastungsfähigkeit auf die Probe zu stellen. (138)
\end{abstract}

Durch diese Passage wird Fischers Frustration inszeniert, aber zugleich wirkt sie auch seltsam unfokussiert. Der Auslöser für diese Frustration oszilliert zwischen einem Affen, der hier schlicht ,das Tier ' ist, dem Aufwand der technischen Ausrüstung und der für das Experiment erforderlichen Geduld und ,Belastungsfähigkeit‘. Julia Fischer als Feldforscherin bleibt daher in Affengesellschaft größtenteils affektiv unbelichtet.

Was Julia Fischer mit ihren ausführlicheren Überblicken über die Kognitionsforschung mit Affen jedoch darstellt, ist die kollektive Forschungsarbeit eines Projektes: Sie gibt wieder, wer wann wozu mit Affen im Kontext des von ihr geleiteten Projektes, aber auch darüber hinaus, zu den ihr wichtigen Themen geforscht hat. So wird vor allem von Experimenten und Freilandbeobachtungen berichtet, die Fischer nicht selbst angestellt hat. Dadurch tritt ein kollektives ,Wir' als protagonistische Gestalt der Primatologie hervor. Fischer und Hammerschmidt verlassen bspw. nach erster Inaugenscheinnahme des Ortes Simenti den Senegal wieder und überlassen das Errichten der Forschungsstation und die Habituation der Affen „Dietmar Zinner, ein[em] erfahrene[n] Pavianforscher aus unserer Arbeitsgruppe“ (69). Doch im Folgenden ist von „wir“ und „uns“ die Rede, wenn es um die Vorgänge rund um das Camp geht (70). Naturgemäß ist dieses

\footnotetext{
${ }^{253}$ Playback-Experimente sind Hörreaktionsexperimente, bei denen zunächst eine Lautäußerung oder ein Geräusch der Tiere aufgenommen und ihnen dann im zweiten Schritt vorgespielt wird die Tonaufnahme wird also dem Testsubjekt, zurückgespielt ${ }^{\star}$.
} 
,Wir ‘ affektbereinigt, denn Fischer erzählt hier wesentlich von den Forschungserfahrungen anderer. Diese anderen sind hinsichtlich des von ihnen erzeugten wissenschaftlichen Gehaltes und nicht als Akteure oder Akteurinnen der Feldforschung vor Ort oder als Affektsubjekte wichtig.

Fischers Masterstudierende, Doktorierende und Postdoktorierende werden ebenso mit den eigenen Forschungsprojekten angeführt wie Wissenschaftler/ innen anderer Universitäten und historische Experimente. ${ }^{254} \mathrm{Im}$ Zentrum dieses Referenzsystems steht aber „unser[] Projekt[]“(81), welches die Basis für die Forschung am Lehrstuhl für Kognitive Ethologie in Göttingen liefert. Die primatologische Forschung, die Fischer in Affengesellschaft porträtiert, ist nicht die einer einzelnen Feldforscherin, die unter persönlichen Entbehrungen eine Station gründet, aufbaut und jahrelang vor Ort Affen beobachtet und sich mit Kolleg/innen, Doktorand/innen und einheimischen Nachbarn und Nachbarinnen auseinandersetzen muss. Die Forschung ist - zumindest dieser Darstellung nach - ein kollektives, kommunikatives und interdisziplinäres, professionalisiertes Unterfangen geworden. Emotionen haben bei der Darstellung einer solchen kollektiven Forschungsarbeit keinen Platz. Denn es stehen zu bestimmten Erklärungsmodellen zugehörige Einzelstudien, Methoden und Hypothesen im Vordergrund. Die Praktiken des Feldforschens selbst sind im besten Fall von anekdotischem Unterhaltungswert für ein Laienpublikum, für den primatologischen Wissensstand, den Fischer wiedergibt, aber weniger entscheidend als z. B. diverse Laborexperimente.

\subsubsection{Affekte: Professionalisierung und Integrität}

Eine solche Strategie, die im Sinne eines Zusammentragens des relevanten Wissens über Affen aus der Affenforschung die Erfahrungen, Beobachtungen und Ergebnisse anderer anhäuft, stellt die Autorin Fischer vor ein Problem der ,Authentizität': Ihr Buch verspricht neben ausführlichen Wissensinhalten nämlich, wie bereits zitiert, doch den Bericht von den „Reizen, Herausforderungen und absonderlichen Begebenheiten“ der Freilandforschung und den Eindrücken davon, wie es , unter Affen' sei.

Das Genre der Forschungsmemoiren ist poetologisch der Autobiographie bzw. den Memoiren verschrieben. Fischer begegnet dieser Problematik mit zweierlei: Zum einen verwendet sie häufiger formelhafte Belege des Sehens und Beobachtens oder der anderweitigen Wahrnehmung, sowohl in Bezug auf sich („Einmal sah ich“, 107, „Ich hörte das erste Mal auf einer Konferenz [...] von dieser Studie“, 106) als auch in Bezug auf andere Forschende, deren Beobachtungen sie referiert: „Satsue Mito, eine junge Frau, die angestellt worden war, um Japanmakaken auf der Insel Koshima an die Präsenz von Beobachtern zu gewöhnen, hatte am Strand Süßkartoffeln verteilt. [...]

\footnotetext{
${ }^{254}$ Dass Fischer derart ein ,team player' ist, zeigt sich in Affengesellschaft daran, wie viel Wert sie darauf legt, namentlich sowohl im Haupttext als auch in einer vierseitigen Danksagung ( $A G$, S. 253-256) aufzuführen, wer für welche Studien verantwortlich war und welche Arbeit geleistet hat.
} 
Mito beobachtete, wie ein junges Affenweibchen [...]“ (121, Hervorheb. MS). Diese Belege sollen in ihrer Anekdoten- und Formelhaftigkeit die ,Wahr- und Welthaftigkeit ${ }^{*}$ der Beobachtungen belegen. ${ }^{255}$ Sie sind jedoch überwiegend ,Beobachtungen zweiter Ordnung ' und entstammen als solche dem Paradigma einer entemotionalisierenden Verwissenschaftlichung und nicht dem Erfahrungsparadigma der Forschungsreise. Es lässt sich sogar behaupten, dass Fischers Affengesellschaft in jenem Sinn als Beobachtung zweiter Ordnung funktioniert, wie ihn Niklas Luhmann für das Wissenschaftssystem identifiziert:

Das Vermittlungsinstrument, das die strukturelle Kopplung der Beobachtung erster und zweiter Ordnung sicherstellt, sind Publikationen, die in der Perspektive erster Ordnung, als Texte, produziert und gelesen werden, aber zugleich zum Durchblick auf die Beobachtungsweise anderer Wissenschaftler (und reflexiv dann auch auf die eigene) führen und erst darin ihren eigentlich wissenschaftlichen Sinn gewinnen. ${ }^{256}$

So ist Affengesellschaft ironischerweise gerade durch die Abkehr vom Erfahrungsparadigma der Forschungsreise und die Hinwendung zu den Operationen wissenschaftlicher Produktion - dem „Referieren des Forschungsstandes und Zitieren anderer Publikationen“257 - trotz seines populären Stils viel eher Teil des heutigen Wissenschaftssystems als andere primatologische Forschungsberichte.

Zum anderen ist sich Fischer der Tatsache bewusst, dass das Genre, auf das sie zurückgreift, um von der Primatologie zu erzählen, ein empfindendes erzählendes Subjekt ins Zentrum rückt. Dieses scheidet einen Text wie A Primate's Memoir von Michael Tomasellos A Natural History of Human Morality, obwohl beides Texte sind, die primatologische Erkenntnisse popularisieren. Um dieser Konvention gerecht zu werden, lässt Fischer jene eigenen Erfahrungen einfließen, die affektive Färbungen tragen. Sie sind jedoch bis auf sehr wenige Ausnahmen nicht aus der Forschungstätigkeit vis-à-vis der Affen gewonnen und bleiben häufig ebenfalls hinter dem Anspruch der Anekdote, pointiert so unterhaltsam wie anschaulich und geistreich zum ,Charaktergemälde' beizutragen, zurück. Neben den oben bereits angeführten touristischen Eindrücken, die eine Affizierung durch Landschaften vermitteln, sind es vor allem Konferenzerfahrungen und Erfolge der eigenen Forscherinnenbiographie, die Fischer als ein solches empfindendes Erzählsubjekt in Erscheinung treten lassen. „[W]ie vom Donner gerührt“ (47) ist Fischer, als sie im Anschluss an einen Vortrag über die Ergebnisse ihrer Promotionsarbeit auf einer internationalen Konferenz in den USA von Dorothy Cheney und Robert Seyfarth angesprochen und zur Leitung der Forschungsstation in Botswana aufgefordert wird: „Es war ein Geschenk des Himmels“ (48). Auf einer Konferenz in Bali trifft sie auf die renommierte Schimpansenforscherin Sarah, Sally“ Boysen und starrt

\footnotetext{
${ }^{255}$ Schlaffer verweist denn auch darauf, dass es im Vergleich mit anderen Kurzformen Eigenart der Anekdote sei, ,den Anspruch auf Faktizität und die Anlehnung an lokal oder historisch bekannte Personen" zu beanspruchen. Schlaffer: Ankedote, S. 87.

${ }^{256}$ Luhmann, Niklas: Die Kunst der Gesellschaft. Frankfurt a. M. ${ }^{9} 1997$, S. 105.

${ }^{257}$ Luhmann: Die Kunst der Gesellschaft, S. 106.
} 
diese „fasziniert“ (106) an: „,eine groß gewachsene, selbstbewusst auftretende Frau, die ein auffallend gemustertes Kleid trug“ (106 f.). Die Information über das Aussehen Boysens ist irrelevant; es geht Fischer an dieser Stelle nur um eine Studie Boysens, auf die sie anschließend rekurriert. Auf einer anderen Konferenz hält die Primatologin Sue Savage-Rumbaugh einen Vortrag, der zwei hinter Fischer den Saal verlassende Verhaltensgenetiker veranlasst, sich darüber zu unterhalten, ,dass die Affenforscher offensichtlich den Verstand verloren hätten. Ich konnte es ihnen nicht verdenken“ (193). Fischer erinnert sich auch ,lebhaft an einen typischen [Peter] Marler-Auftritt bei einem Symposium“ (195) am Institut für Verhaltensbiologie der Freien Universität Berlin, und gibt an, bei einer Einladung bei Marler zu Hause habe dieser sie freundlich angeblinzelt und gesagt, ,dass ich als Schülerin von Robert und Dorothy ja irgendwie seine wissenschaftliche Enkelin sein. Eine schöne Vorstellung“"(195).

Wie sich in diesen Zitaten, in denen es um wissenschaftliche Anerkennung geht, zeigt, neigt Fischer bei den wenigen Verweisen auf im weitesten Sinne affektives Erleben in der Wissenschaft ebenfalls zum Sprachklischee, zur Formelhaftigkeit, die einen konventionalisierten Eindruck abruft: Ob , vom Donner gerührt oder ,kalter Schweiß", Fischer bedient sich eines Affektphrasenrepertoirs, ohne es individuell auszugestalten. Besonders bemerkenswert ist dieser Rückgriff auf Phraseologismen und tradierte Affektklischees auch hinsichtlich zweier Passagen, die jeweils für sich starke Affektauslöser beinhalten (könnten): Die Begegnung mit der Fremdwahrnehmung der Primatologin im Senegal und die Ereignisse des 11. September 2001.

Fischers ,,persönliches Highlight im Senegal“ ist „der Versuch, Fahrzeugpapiere für unser Auto zu bekommen“ (81). Zu diesem Zweck hat das Projekt mit Monsieur Seydi einen einheimischen Berater und Vermittler engagiert, der die bürokratischen Vorgänge für die Europäer/innen vereinfachen soll. Fischer trifft ihn im besten Hotel am Platz; Seydi entwickelt den Plan, mehrere ,hochrangige Persönlichkeiten“ (82) aufzusuchen. „Missbilligend betrachtete er meine Kleidung“, schreibt Fischer dann: „Ob ich kein Kostüm dabeihätte? Keine schicken Schuhe? So könne er sich mit mir nicht sehen lassen“ (82). Obwohl Fischer später angesichts dieser Episode darauf verweist, dass man es ,[a]ls Frau [...] im Senegal mit seiner patriarchalen Struktur nicht leicht“ (85) habe, ist sie hier doch getroffen und schreibt: „Ich schämte mich sehr“ (82). Diese Scham entsteht vor allem aus einem Grund:

\footnotetext{
Ich schämte mich sehr, hätte ich nach Jahren in Afrika doch wirklich wissen müssen, dass der Look der Feldforscher im Straßenleben vollkommen inakzeptabel ist. Die meisten Einheimischen sind wie aus dem Ei gepellt, wenn sie ihren Geschäften nachgehen, und die Nachlässigkeit der weißen Entwicklungshelfer, Forscher oder Großwildjäger wird mit einiger Irritation zur Kenntnis genommen. Dabei sah ich nun nicht schlampig oder dreckig aus, aber warum sollte ich in High Heels über die Sandpiste vor dem Brazzerade stolpern? Ich hätte es besser wissen sollen. (82, Hervorheb. im Original)
}

Fortan hat sie ,,auch immer einen guten Anzug und schicke Schuhe mit, wenn ich nach Dakar fahre“ (85), und lässt ihre Mitarbeitenden mit einem Seminar auf die nicht affen-relevanten Seiten des Forschungsaufenthaltes im Senegal vorbereiten. Der Rest der kleinen Erzählung ist ein kafkaesk anmutender Behördenlauf, den 
Fischer jedoch eher belustigt wiedergibt als affektiv ausgestaltet. Diese Episode ist aber eines der wenigen vollständigen Mini-Narrative in Affengesellschaft, weil es von der Aufnahme des Versuchs bis zum Erlangen der Papiere durchgängig erzählt, was geschehen ist, und auch Pointen setzt. ${ }^{258}$ Die zweifache Erwähnung des ,Ich hätte es besser wissen sollen/müssen“ verweist dabei darauf, wovon sich Fischer affizieren lässt: von einem eigenen Fehler, einer Nachlässigkeit, die auf mangelnde Professionalität hinweisen könnte.

Die zweite Passage betrifft die Koinzidenz, dass Fischer mit den Primatolog/ innen Josep Call und Juliane Kaminski vom Leipziger Max-Planck-Institut für Evolutionäre Anthropologie ausgerechnet am 11. September 2001 einen durch die Fernsehsendung Wetten daß...? bekannt gewordenen Hund in Bochum aufsucht, der seine Spielzeuge beim Namen erkennen kann:

Kurz vor drei, als wir gerade das Auto abgestellt hatten, hörten wir im Autoradio die Eilmeldung, dass ein Flugzeug ins World Trade Center geflogen war. Ich malte mir aus, wie ein idiotischer Hobbypilot mit seiner Cessna in einen der Türme geknallt war, und dachte nicht weiter über die Meldung nach. Drinnen wurden wir von Suse [die Hundehalterin Susanne Baus] und ihrer Familie sehr herzlich empfangen. [...] Wir saßen bei diesen uns damals noch völlig fremden Leuten und starrten ungläubig auf die Bilder von $\mathrm{CNN}$, die immer wieder zeigten, wie die Flugzeuge ins World Trade Center rasten und wie die beiden Türme später in sich zusammensanken. Der Rest ist Geschichte. (220 f.)

Tatsächlich ist dieses weltgeschichtlich einschneidende Ereignis mit der Floskel „Der Rest ist Geschichte“ für Julia Fischer abgehandelt. Im Folgenden geht es um die Zusammenarbeit mit Susanne Baus und ihrem Hund Rico. Auch hier scheint vor allem ein wissenschaftliches Ethos wichtig. Dieses erlaubt zwar punktuell, ungläubig bei Fremden auf dem Sofa die CNN-Berichterstattung zu sehen, jedoch nicht, an dieser Stelle davon zu berichten, welche Bedeutung das Ereignis hat oder detailliert auszumalen, welche affektive Reaktion das Erzählsubjekt darauf erlebt. Stattdessen steht im Zentrum, „ob Rico die Spielzeuge wirklich beim Namen kannte oder ob er - ähnlich wie das berühmte Pferd, der Kluge Hans - auf für menschliche Beobachter unmerkliche Zeichen seiner Besitzerin achtete“ (221), also eine Frage der wissenschaftlichen ,Sauberkeit‘ im Umgang mit Experimenten.

Die Affektivität, die in Affengesellschaft zur Darstellung kommt, ist somit nicht an einzelne Forschungserlebnisse mit Affen im Feld, sondern an ein Selbstverständnis als Wissenschaftlerin gebunden, das mit persönlicher Professionalität und wissenschaftlicher Objektivität verknüpft ist. Auch die eine Schilderung von Affektivität im Feld unterstützt dies: Fischers Reaktion - kalter Schweiß auf der Stirn - bezieht sich auf das (Nicht-)Funktionieren des konzipierten Experiments, also auf die Durchführbarkeit ihres wissenschaftlichen Projektes unter möglichst

\footnotetext{
${ }^{258}$ So z. B. dass die von ihr in der Not ausgewählte Kleidung - „eine schokoladenbraune Kombination mit Glitzer-Applikationen und einer großen Seidenschleife als Gürtel“ ( $A G, \mathrm{~S}$. 82) und „eine passende Plastik-Handtasche und altrosa Pumps“ (S. 83) - vom Leiter der Nationalparkbehörde als ,äußerst elegant“ (S. 84) bezeichnet wurde.
} 
stabilen Bedingungen. Im Bild der Affen, die mit ihr einen Test ihrer Belastungsfähigkeit durchführen, steht Fischers Eignung als Primatologin zur Disposition. Es ist diese Infragestellung, die ihr den Schweiß auf die Stirn treibt.

\subsubsection{Der Primatologe als Afrikareisender: Volker Sommers Schimpansenland (2008)}

\subsubsection{Stil: Die Afrikareise}

Auch Volker Sommer wählt für seine Forschungsmemoiren einen saloppen Stil, dessen umgangssprachliche und phraseologische Züge Fischers Affengesellschaft sogar noch hinter sich lassen. Hier ist man „splitterfasernackt“ (11), ,pitschnaß“, es ,plumpst“ und ,plantscht“ (21) oder ist „krachbunt[]“ (28). Der Dschungel des Gashaka-Nationalparks ist „kein Präsentierteller“ (16), Affenkot - oder „Kacke“ (103) - dafür eine „Goldgrube für Genetiker“ (18). Wegen der „Maispampe“ (ein einheimisches Grundnahrungsmittel) und der „hygienischen Ultramonster“ (einheimische Köche) ,mausert sich [der Dschungeltrip] zur Abmagerungskur“ (19); es gibt „Kabinettstückchen“ (22), „Mutter Erde“ (24), „whiskasverwöhnte Miezen“ und „schwarzweiße Camptiger“ (42), „obere[] Chargen“ (47), ,jungfräuliche[] Besucher“ (56) und „Publikumsknüller“ (74). Man muss „kleine Brötchen backen“ (31) und „wo das Gewässer ein bißchen tiefer ist, pißt und kackt es sich bequem in die Fluten“ (177). Die Wahl eines solchen umgangssprachlichen Erzählstils soll sichtbar ebenfalls den Bericht aus dem Feld nahbar gestalten und Sommer als Erzähler unterhaltsam charakterisieren: Hier spricht nicht der Londoner Professor, sondern der Abenteurer aus Nordhessen. ${ }^{259}$ Diese Ausrichtung auf die Vermittlung von wissenschaftlicher Forschung als ,Abenteuer Wissenschaft' geht auch einher mit Volker Sommers Bewusstsein für die ,Gemachtheit' evolutionärer Narrative und primatologischen Wissens. Bereits in seinem Vorwort „Notiz aus dem Dschungel“ (9) verweist er darauf, dass ,,[f]ür einen Evolutionsbiologen“ (9) wie ihn „Primaten Informanten über die Geschichte der Menschheit [sind]: die Millionen Jahre alte Story, die wir mit anderen Kreaturen teilen“ (9). Wie in einem programmatischen Aufsatz ${ }^{260}$ gefordert, und auch in Schimpansenland diskutiert (207), sieht Sommer das Verhältnis von Primatolog/innen und Affen dem zwischen Ethnolog/innen und Informant/innen strukturell analog. Dass Sommer für seine Forschungsmemoiren Schimpansenland die Form eines Afrikareiseberichts wählt, ist vor diesem Hintergrund problematisch. Unter den (Forschungs-)Reiseberichten nimmt der Afrikareisebericht eine besondere

\footnotetext{
${ }^{259}$ Sommer verortet sich zwar wissenschaftlich und disziplinär mit seinem Lehrstuhl am University College in London, legt jedoch Wert darauf, seine ländliche nordhessische Herkunft hervorzukehren. Vgl. SP, S. 121 ff.: „Im Märchenwald der Brüder Grimm“.

${ }^{260} \mathrm{Vgl}$. Sommer, Volker: The Anthropologist as a Primatologist. Mental Journeys of a Fieldworker. In: Jeremy MacClancy/Agustín Fuentes (Hg.): Centralizing Fieldwork. Critical Perspectives from Primatology and Biological Anthropology in the Lens of Social Anthropological Theory and Practice. New York/Oxford 2011, S. 32-48.
} 
Funktion ein, wie verschiedentlich bereits untersucht wurde. ${ }^{261}$ Die Afrikareise diente deutschen Akademikern vor allem im 19. Jahrhundert als Qualifikation für naturwissenschaftliche bzw. ethnologische Forschung, wie Cornelia Essner herausgearbeitet hat:

Die akademischen Afrikareisenden, die die traditionelle [...] wissenschaftliche Arbeitsteilung zwischen Empirie und Theorie in ihrer Person aufhoben, indem sie beide Rollen chronologisch lebten, trugen damit in besonderem Maße dazu bei, die induktive Methode des ,naturwissenschaftlichen Zeitalters' auch in den Wissenschaftsgebieten durchzusetzen, die sich mit den ,sozialen Facta' beschäftigten. Beobachtungen, Erlebnis und Erfahrung, zusammengetragen durch die weitgehende Willkürlichkeit des Reisens, wurden zu einer kognitiven Qualität, die der theoretischen Reflexion als überlegen galt. ${ }^{262}$

Die primatologische Feldforschungsreise scheint somit per se in der Tradition dieser Art der Forschungsreise des 19. und frühen 20. Jahrhunderts - und ihrer Verschriftlichung - zu stehen. Schließlich vollzieht sie ebenfalls die Zusammenführung von Empirie und Theorie in einer Person und bietet mit ihren Beobachtungen, Erlebnissen und Erfahrungen der theoretischen Reflexion das Datenbeet, auf dem normative Wissensbestände überhaupt erst wachsen können. Jedoch verweist Essner auch auf die spezifisch ideologische Grundierung dieser Tradition in Deutschland: Die Reisenden erwiesen sich ,[i]n besonderem Maße als Repräsentanten eines konservativen Denkens, dessen Charakteristikum [...] die Betonung des Konkreten gegenüber dem Abstrakten ist“".263

Schimpansenland ist nicht einfach ein Bericht der Reise eines Primatologen zu einer Schimpansenpopulation im nigerianischen Gashaka-Gumti-Nationalpark.

\footnotetext{
${ }^{261}$ Vgl. u. a. Miller, Christopher L.: Blank Darkness. Africanist Discourse in French. Chicago/ London 1985; Zantop, Susanne: Colonial Fantasies. Conquest, Family, and Nation in Precolonial Germany. 1770-1870. Durham/London 1997, S. 198 ff.; Curtin, Philip D.: The Image of Africa. British Ideas and Action, 1780-1850. London 1965, S. 14 ff.; Berman, Russell A.: Enlightenment or Empire. Colonial Discourse in German Culture. Lincoln 1998, S. 79 ff.

${ }^{262}$ Essner, Cornelia: Deutsche Afrikareisende im neunzehnten Jahrhundert. Zur Sozialgeschichte des Reisens. Stuttgart 1985, S. 107.

${ }^{263}$ Essner: Deutsche Afrikareisende, S. 107. Essner bezieht sich hier auf Karl Mannheims Morphologie des konservativen Denkens. Mannheim sieht als einen der wesentlichen Charakterzüge des konservativen Erlebens und Denkens, ,das Sichklammern an das unmittelbar Vorhandene, praktisch Konkrete“, in der Folge ein „,neuartiges, gleichsam emphatisches Erleben des Konkreten“: „Konkret zu erleben, konkret zu denken, bedeutet von nun an eine besondere Verhaltensweise, ein ausschließliches Wirkenwollen in jener bestimmten unmittelbaren Umwelt, in die man hineingestellt ist - eine radikale Abneigung gegen jedes ,Mögliche" und ,Spekulative““. Mannheim, Karl: Konservativismus. Ein Beitrag zur Soziologie des Wissens. Hg. von David Kettler, Volker Meja und Nico Stehr. Frankfurt a. M. ${ }^{2}$ 1984, S. 112 f., Hervorheb. im Original. Im Gegensatz zum progressiven Denken, das aus dem Bewußtsein für das Mögliche die Struktur der Welt zu verändern sucht, interessiert sich das konservative Denken nach Mannheim nicht für systematische Veränderungen, sondern nur für die Ersetzung des Konkreten durch das Konkrete. Zu den damit einhergehenden Kulturtheorien des deutschen kolonialen Denkens siehe auch Gründer, Horst: „...da und dort ein junges Deutschland gründen“. Rassismus, Kolonien und kolonialer Gedanke vom 16. bis zum 20. Jahrhundert. München 1999.
} 
Volker Sommer macht explizit das Phantasma Afrika ${ }^{264}$ zum Aufhänger seines Affenbuches und reiht seinen Text schon damit deutlicher als andere primatologische Feldforschungstexte in die Tradition des Afrikareiseberichtes ein. Er sieht sich sogar selbst explizit in der Tradition der großen Afrikareisenden: „Unterwegs sind wir, weil ich an der Universität London Evolutionsbiologie unterrichte und bei meinen Forschungen Primaten im Mittelpunkt stehen. In diesen entlegenen Winkel verschlägt es mich somit wegen des Affentheaters im umliegenden Dschungel“" (12), so begründet Sommer seine Anwesenheit in Gashaka. Nach langjähriger Forschung in Indien mit Hanuman-Languren ${ }^{265}$ erschließt sich für ihn mit der gerade erst als genetische Subgruppe klassifizierten Schimpansenpopulation in Nigeria noch einmal eine neue Welt. Hier kann er sich als wahrer Forschungsreisender fühlen, der Neuland für die Wissenschaft erschließt:

Ich fühle mich wie frühere Forschungsreisende nach einem , first contact ${ }^{\star}-$ so, als wären wir zum ersten Mal einem sagenumwitterten Volksstamm begegnet. (25)

Von hier an darf ich Livingston spielen: so tun, als wäre ich ein weißer Entdecker im 19. Jahrhundert, der mit heimischen Trägern das unbekannte Afrika erkundet. (105)

Man könnte an diesen Formulierungen eine ironische Haltung zur Rolle des Primatologen als Afrikaabenteurer ableiten. Doch Sommer ist nur allzu gern bereit, die Topoi des Genres affirmativ abzuarbeiten und sich eine Identität als konservativer ,Afrikakenner' $\mathrm{zu}$ formen. ${ }^{266} \mathrm{Er}$ beginnt mit einer Einbaumszene mit einem nackten ,Eingeborenen“ (11 f.), lässt seine nigerianischen Assistenten sein schweres Gepäck tragen (13 f., 105 ff.) und begründet dies mit deren Körperstärke („die Kräfte von Büffeln“, 14), setzt sich in der Pose des Anthropologen des 19. Jahrhunderts im Dorf, ,auf einer dicken Wurzel nieder und studier[t] das Leben und Treiben“ (27), beschwert sich über das einheimische Essen (15, 42 ff.) und über „gewisse afrikanische Eigengesetzlichkeiten“ (44). In der Pose des Abenteurers marschiert Sommer seinem Tross bei der Erkundung des NationalparkGebietes voraus - muss sich aber von seinen einheimischen Mitarbeitern beschützen (104) oder den Weg freischlagen lassenlassen (106 f.). Wie seine Vorbilder jagt

\footnotetext{
${ }^{264}$ Vgl. Miller: Blank Darkness, S. XI sowie $15 \mathrm{ff}$.

${ }^{265}$ Der Bericht von dieser langjährigen Feldforschung findet sich in der Publikation Heilige Egoisten. Stärker an den Texten Sarah Blaffer Hrdys u. a. orientiert wird dabei soziobiologische Modellbildung, Affenwissen und Kulturelles ebenso aufbereitet wie zusammengefasste Forschungsbeobachtungen und -ergebnisse. Sommer, Volker: Heilige Egoisten. Die Soziobiologie indischer Tempelaffen. München 1996.

${ }^{266}$ Zur Funktion Afrikas als Raum nationaler und wissenschaftlicher Identitätskonstruktionen vgl. auch Shah, Mira: Richard Kandts Reisebericht ,Caput Nili‘. Die Konstruktion moderner Identität im Raum des Anderen. In: Tim Mehigan/Alan Corkhill (Hg.): Raumlektüren. Der Spatial Turn und die Literatur der Moderne. Bielefeld 2013, S. 167-188. Flitner sieht spezifisch den Tropenwald als „Bühne einer prekären Identitätssuche der Deutschen“ auch nach 1945: Flitner, Michael: Vom ,Platz an der Sonne“ zum ,Platz für Tiere'. In: Ders. (Hg.): Der deutsche Tropenwald. Bilder, Mythen, Politik. Frankfurt a. M./New York 2000, S. 244-262, hier S. 245.
} 
Sommer seine Expedition einen unwirtlichen Berg hinauf zum Gipfel, obwohl ihnen die Vorräte ausgehen (109 ff.). ${ }^{267}$ Muss er einmal mit dem „Buschtaxi“ (132) fahren, kauft er lieber „mehrere Sitze für mich allein, damit es nicht gar so eng ist“" (132). Wie stark Sommer dabei von der Kolonialgeschichte und -imagination geprägt ist, zeigt sich auch daran, dass die Unterhaltung afrikanischer Menschen nicht als Gespräch, sondern pejorativ als „Palaver“ $(26,46)$ bezeichnet wird. ${ }^{268}$

Deutsche Kolonialgeschichte, gerade diejenige vor Ort, hat Sommer zwar vor Augen. Er scheint sie jedoch als historisches Kolorit zu betrachten, an das sich gestalterisch anknüpfen oder das sich im Sinne pragmatischer Überlegungen ignorieren lässt. So bereitet ihm das Angebot seines Mitarbeiters Hammaunde, sein Gepäck für ihn zu tragen ,gemischte Gefühle [...]. Hammaunde ist von kleiner Statur, und man will ja nicht als Schwächling erscheinen. Die Erinnerung an die Kolonialzeiten tut ihr übriges. Doch Skrupel hin, Skrupel her: Schließlich siegt mein Pragmatismus“ (13). Vorab hatte Sommer bereits bereut, „keinen eigenen Träger für meinen schweren Rucksack engagiert zu haben“ (13). Gerade der Ort Gashaka ist Teil deutscher Geschichte, wie Sommer eher bewundernd als kritisch aufgreift:

[K]aiserliche deutsche Truppen wählten die Siedlung als strategischen Ausgangspunkt, um ihre kolonialen Ambitionen in Westafrika voranzutreiben. Der Fußpfad in den Dschungel wurde ebenso von deutschen Soldaten angelegt wie ein bis auf die Grundmauern zerfallenes Fort, das sich hinter dem Dorf auf einer Anhöhe erhebt. Das nicht weit entfernte ,Jeremani“ ist zu Ehren von „Germany“ benannt, und die Schule wurde neben dem wohl Tapferkeit symbolisierenden Grab eines kaisertreuen Kämpfers angelegt. (27)

Beim Aufbau einer Solaranlage in Gashaka werden besonders fleißige Träger später mit ,als Prämie eine[r] gebrauchte[n] Bundeswehrjacke, mit Flagge auf dem linken Oberarm“ (132) belohnt. Für Sommer ist dies wieder Anlass zu kolonialanekdotischen Ausschmückungen:

Trosse deutscher Soldaten marschierten zuletzt vor 87 Jahren über den gleichen Fußweg, bevor die Kolonie an die Briten verlorenging. Jetzt ist eine schwarz-rot-goldene Armee zurïck und bewegt sich auf eine Höhe zu, auf deren linkem Kamm sich zu allem Überfluß das illegale Gehöft „Gilda Hitla“ befindet, das „Haus Hitler“. (132) ${ }^{269}$

\footnotetext{
${ }^{267}$ Die hungernde Expedition mit widerwilligen Trägern in widerständiger Natur findet sich bspw. bei Du Chaillu (EA, S. 78 ff.) sowie in Kandt, Richard: Caput Nili. Eine Empfindsame Reise zu den Quellen des Nils. Berlin ${ }^{5} 1921$, S. 429 ff.; Stanley, Henry Morton: In Darkest Africa; Or, The Quest, Rescue, and Retreat of Emin, Governor of Equatoria. New York 1890, S. $216 \mathrm{ff}$.

${ }^{268}$ Zur kolonialen Geschichte des Begriffs ,Palaver" und seinem Einsatz zur Diskriminierung einheimischen Sprechens siehe Shah, Mira: A Word. Palaver and its transferal residues. In: Word and Text. A Journal of Literary Studies and Lingustics IV/2 (2014), S. 67-83.

${ }^{269}$ Hitler und die deutsche Kolonialmacht bringt Sommer schon in der vorangegangenen Textstelle in Verbindung, wenn es heißt, den Deutschen wurde ,selbst nach der Niederlage gegen die Briten Ende des Ersten Weltkrieges die Treue gehalten. Denn bis heute nennt sich die dort lebende Familie ,Hitla““ (SP, S. 27).
} 
Historische Sorgfalt, sodass der heute nigerianische Teil der deutschen Kolonie Kamerun strenggenommen nicht an die Briten ,verlorenging ' oder dass die deutsche Schutztruppe nicht unter einer schwarz-rot-goldenen Fahne marschierte, wird von Sommer hier zugunsten eines Blicks auf die lokale deutsche Kolonialgeschichte vernachlässigt, ${ }^{270}$ der zwischen Nostalgie für zivilisatorische Errungenschaften (das Anlegen eines Fußpfades und der Bau eines Forts), Mythologie (Tapferkeit) und Sensationslust (Hitler) schweift.

Besonders die Nostalgie steht in Zusammenhang mit Sommers eigener Aufgabe vor Ort. Der Text liegt durch sein explizites Naturschutzanliegen auf der Linie westlicher Zivilisierungsmissionen. Eine solche Zivilisierungsmission bedingt, wie Jürgen Osterhammel definiert, die Vorstellung einer Position zivilisatorischer Überlegenheit in einer umfassenden weltgeschichtlichen Fortschrittsbewegung, aus der ,das Recht und die Pflicht ab[geleitet] [wird], dieser Bewegung zu universaler Entfaltung zu verhelfen oder zumindest in konkreten Notständen durch aktives Eingreifen für ein Minimum an zivilisatorischer Normerfüllung zu sorgen“. ${ }^{271}$ Naturschutz kann als eine solche Zivilisierungsmission betrachtet werden, denn das Konzept erfüllt zwei wesentliche Bedingungen: Erstens beinhaltet die Idee der schützenswerten Natur die Vorstellung, „,dass sich die Überlegenheit bestimmter Rechtsnormen vor konkurrierenden anderen argumentativ erweisen lässt“, ${ }^{272}$ und dass sie ,,auch dann als universal gültig betrachtet werden, wenn sie in einem bestimmten kulturellen Zusammenhang, zumeist dem okzidentalen, entstanden sind“".273 Zweitens wird es als ,unter gewissen Umständen erforderlich und legitimierbar“ betrachtet, ,,solchen Maßstäben von Zivilisiertheit durch Intervention ,in die Angelegenheiten anderer' zur Verwirklichung zu verhelfen“. 274

Mit der Einschreibung in die Geschichte der Zivilisierungsmission, tritt Sommer aber auch das rassistische Erbe des Afrikareiseberichts an. So schreibt Sommer: „verblüffend, wieviel schärfer Schwarzafrikaner sehen können - dank

\footnotetext{
${ }^{270}$ Zum durch ,[a] number of well-publicized incidents of brutality by the colonial government [...][,] arousing indignation even within Germany“, gekennzeichneten deutschen Kolonialismus im Schutzgebiet Kamerun siehe Friedrichsmeyer, Sara/Lennox, Sara/Zantop, Susanne: Introduction. In: Dies. (Hg.): The Imperialist Imagination. German Colonialism and Its Legacy. Ann Arbor 1998, S. 1-29, hier S. 11 f.

${ }^{271}$ Osterhammel, Jürgen: ,The Great Work of Uplifting Mankind‘. Zivilisierungsmission und Moderne. In: Boris Barth/Jürgen Osterhammel (Hg.): Zivilisierungsmissionen. Imperiale Weltverbesserung seit dem 18. Jahrhundert. Konstanz 2005, S. 364-425, hier S. 363. Im Deutschen, das sich traditionell im Kolonialdiskurs von ,Kultur vs. Zivilisation“ lang auf der Seite der Kultur verortet hat, handele es sich bei der Zivilisierungsmission um einen Neologismus, so Barth/ Osterhammel, der sich jedoch definitorisch auf historische und gegenwärtige Formen anwenden lasse. Vgl. Barth, Boris/Osterhammel, Jürgen: Vorwort. In: Dies. (Hg.): Zivilisierungsmissionen. Imperiale Weltverbesserung seit dem 18. Jahrhundert. Konstanz 2005, S. 7-11, hier S. 7.

${ }^{272}$ Barth/Osterhammel: Vorwort, S. 9.

${ }^{273}$ Barth/Osterhammel: Vorwort, S. 9.

${ }^{274}$ Barth/Osterhammel: Vorwort, S. 9.
} 
besonderer Augenpigmente“ (13). ${ }^{275}$ Das Verhalten einzelner Einheimischer wird generalisiert, nicht nur auf Nigerianer/innen, sondern gleich den ganzen „Schwarzen Kontinent", wie Sommer Afrika wiederholt nennt (31, 58, 125). Gerade die Opposition schwarz/weiß hat es Sommer angetan. Er erläutert gern, warum sein nigerianischer Doktorand „es super [findet], mit Weißen arbeiten zu können“ (32), oder weshalb man sich als Weißer in Afrika alles erlauben könne (,Daß die weiße Hautfarbe geradezu vergötzt wird“, 180). An einer Stelle heißt es sogar, in der deutschen Presse sei Nigeria ,schon mal und durchaus zutreffend als ,Negeria“ bezeichnet" (127) worden.

Sommers in der Primatologie wirksames theoriebildendes Talent wird in Schimpansenland darauf aufgewendet, ,Afrika" kultur- und gesellschaftstheoretisch für sein Publikum zu erläutern. So wenn es ebenfalls in der Tradition deutscher Kolonialkultur um Arbeitseifer, Fleiß und Qualitätsarbeit geht (,Wie in Nigeria die Uhren gehen“, 46) ${ }^{276}$, um hierarchisches Denken, das er allein Afrikanern zuschreibt, ${ }^{277}$ oder um einen Mangel an Natur- und Wissensbegeisterung:

An spektakulärer Natur zeigen Afrikaner normalerweise wenig Interesse. (109)

Aus Neugier und Interesse einfach etwas wissen zu wollen ist ein fremdes Konzept. (48)

Die Wertewelt der Weißen - Abenteuer erleben zu wollen, Unbekanntes zu erforschen ist in Afrika absurd. (111)

\footnotetext{
${ }^{275}$ Eine Behauptung, die sich nur auf einen ophthalmologischen Aufsatz vom Beginn des 20. Jahrhunderts zurückverfolgen lässt, aber als Teil einer rassistisch motivierten kolonialen Rhetorik anderweitig zu finden ist. Vgl. Roy, J. N.: The Eyesight of the Negroes of Africa. In: Canadian Medical Association Journal 9/2 (1919), S. 144-154; Stapleton, Timothy: African Police and Soldiers in Colonial Zimbabwe 1923-80. Rochester/Woodbridge 2011, S. 66 f.

${ }^{276}$ Zur ,Erziehung zur Arbeit“ als „Fundamentalsatz der deutschen Kolonialideologie und Kolonialpädagogik" vgl. Gründer: ...da und dort ein junges Deutschland gründen, S. 227. Osterhammel legt dar, wie sich Afrikaner/innen als „Objekte der Zivilisierung“ im Diskurs der Zivilisierungsmissionen veränderten: So wurde ,aus dem Sklaven, den man befreit hatte, der menschenfressende Wilde, der mit Gewalt von seinem mörderischen Treiben abzubringen sei, und aus diesem später unter stabileren kolonialen Verhältnissen wiederum der harmlose, aber faule und nur durch strenge Gängelung zu nützlicher Tätigkeit motivierbare ,Eingeborene““. Osterhammel: Zivilisierungsmission und Moderne, S. 370. Bei Sommer scheinen synchron alle drei Stadien auf: Afrikaner/innen sind noch aus Fesseln der Unmündigkeit zu Befreiende, sind - in diesem Fall - Affenfresser/innen, die ganze Arten ausrotten, und immer wieder harmlose, aber zur Faulheit neigende Subjekte einer fehlgeleiteten Entwicklungspolitik, die sie der Arbeit entwöhnt hat.

${ }^{277}$ „In gesamtgesellschaftlicher Perspektive verhindert die pyramidale Struktur jeden Fortschritt. Denn weder wissen die oberen Chargen Kritik zu würdigen, noch melden die unteren eigene Bedürfnisse an. In Europa werden wir hingegen von klein auf zum Widerspruch ermutigt. Den Unterschied beschreiben Sozialpsychologen als den zwischen ,kollektivistischen " und ,individualistischen ' Gesellschaften. [...] Wenn europäische Ich-Menschen in Afrika auf traditionelle Wir-Menschen treffen, ist Unverständnis somit vorprogrammiert" (SP, S. 47).
} 
Trotz dieser problematischen Zuschreibungen sollte man Sommer nicht voreilig auf die Seite eines ideologischen Rassismus stellen. Seine Haltung entspricht nicht unbedingt dem ,durch Erfahrung unkorrigierbaren Glauben [...], die Existenz anderer sei primär durch eine biologisch determinierte, unveränderliche Inferiorität bestimmt“" ${ }^{278}$ wie Osterhammel Rassismus definiert. Viel eher wird an Sommer jene ,auf kulturelle Merkmale bezogene Arroganz “279 deutlich, die Osterhammel als vereinbar mit den Ideen der Zivilisierungsmissionen und vom Rassismus geschieden sieht. ${ }^{280}$ Auch hier zeigt sich das Erbe einer deutschen Kolonialkultur, das Sommer allein schon durch die Einschreibung in den Naturschutzdiskurs antreten muss: Michael Flitner hat bereits darauf verwiesen, wie stark Naturschutz, besonders jene ökologischen Initiativen zum Schutz des Tropenwaldes, in postkolonialer, postmoderner und postfaschistischer Tradition stehen. ${ }^{281}$ Neben den aus der Frustration des ,Zivilisierenden “ über das offensichtlich zumindest partielle Scheitern des okzidentalen Projektes Naturschutz vor Ort erwachsenden Mentalitätsdiskursen, die sich in jenen Ausführungen über ,de[n] mentalen Kosmos der Afrikaner" (51) darbieten, zeigt sich dies auch in einem Denken von Volk und/ ohne Raum. ${ }^{282}$ Zum einen sieht Sommer, ähnlich wie Flitner dies für Bernhard Grzimeks Reiseberichte aus Afrika belegt hat, die Schimpansen (und andere Tiere) als eine Art ,Volk ohne Raum‘. Stärker als andere Feldforschungsberichte macht Sommer hier verblüffend nah an Grzimek „Bevölkerung, Kolonialismus, Modernisierung, Naturschutz “283 zu seinen Themen: Wegen des Bevölkerungswachstums stehen „die Zeichen insgesamt auf Sturm“ (211). „Nigerianische Frauen“ sind überfertil (,im Durchschnitt 5,3 Kinder“), die „Menschenmassen verdoppeln sich dadurch in nur drei Jahrzehnten“, „Legionen von Landhungrigen“ (211) sind

\footnotetext{
${ }^{278}$ Osterhammel: Zivilisierungsmission und Moderne, S. 420.

${ }^{279}$ Osterhammel: Zivilisierungsmission und Moderne, S. 420.

${ }^{280}$ Osterhammel nimmt mit Blick auf historische Zivilisierungsmissionen wie die des deutschen Kolonialismus und Imperialismus an, dass sich die Zivilisierungsmission, die wesentlich davon ausgeht, das andere im Rahmen einer Fortschrittsbewegung auf eine höhere Entwicklungsstufe gehoben werden können, und Rassismus, der Entwicklungsstufen als biologisch determiniert betrachtet, gegenseitig ausschließen. Nichtsdestotrotz kann Rassismus als Haltung in Reaktion auf die den nur auf Erfolg ausgerichtete Zivilisierungsmissionen zwangsläufig eingeschriebene Frustration der Zivilisierenden angenommen werden. Vgl. Osterhammel: Zivilisierungsmission und Moderne, S. $371 \mathrm{f}$. und den hier folgenden Abschn. 4.4.2.3.

${ }^{281}$ Vgl. Flitner: Vom ,Platz an der Sonne', S. 255. Flitner versteht das Präfix ,post‘ nicht als schlichten Signifikanten historischer Chronologie sondern berücksichtigt die gleichzeitige Kontinuität der entsprechenden Programme des Kolonialismus, Modernismus und Faschismus.

${ }^{282}$ Wie sehr dies als exemplarisch für die Akteure und Akteurinnen von Naturschutzprojekten gelten kann, zeigt Volker Heins Studie des kulturellen Hintergrundwissens und des „Gründlichkeits- und Objektivitätsideal[s] deutscher Naturschützer“" u. a. dahingehend, wie sie Mentalitätsdiskurse schaffen: Heins, Volker: Woran der Wald genesen soll. Zur politischen Theologie von Tropenwalddiskursen. In: Michael Flitner (Hg.): Der deutsche Tropenwald. Bilder, Mythen, Politik. Frankfurt a. M./New York 2000, S. 279-291, hier S. 280.
}

${ }^{283}$ Flitner: Vom ,Platz an der Sonne', S. 253. 
unterwegs. ${ }^{284}$ Den schon zitierten Verweisen auf den deutschen Kolonialismus und seine ,Errungenschaften“ stellt Sommer seine eigenen Modernisierungsversuche $^{285}$ positiv bei, deren Konsequenzen aber gern negativ dar, vor allem in der Verallgemeinerung, Entwicklungshilfe macht faul'.286 Naturschutz ist das bereits paratextuell ausgewiesene Ziel der ,grüne[n] Kampfschrift“ (9 und auf dem Buchumschlag) und wird immer wieder als Motivation angeführt.

Zum anderen präsentiert sich Volker Sommer mit der Form seines Berichts, der Wahl des saloppen Stils und dem Anknüpfen an alte afrikanistische Traditionen als ,Autor des Volkes ‘ - und tatsächlich wimmelt sein Text von Bezügen auf dieses: „Volksstamm“ (25), „Volksseele“ (84), „Volksglauben“ (87), ,Volksrede“ (111), ,Volksmärchen“ (124), „Volkswissen“ (171), „Volksmedizin“ (171), „Volksgesundheit“ (179), „Völkerkunde“ (208), „Volkserziehung“ (225). Diese ,Volkstümelei ‘ zeigt sich nicht nur in Bezug auf seinen Gegenstand ,Afrika ', sondern auch in jenem auf seine (nord-)hessische Heimat. ${ }^{287}$ Diese Heimat, vor allem den „Märchenwald der Gebrüder Grimm“ (121), sieht Sommer als Ausgangspunkt für sein ,Abenteuer Afrika': „Der große Wald erklärt, warum ich dieses Buch über einen anderen Wald in Afrika schreibe“ (123). Auf die Besonderheit einer solchen auch gefühlsqualitativen deutschen Verquickung von identitärem Wald und Naturschutz des Tropenwaldes hat ebenfalls bereits Flitner hingewiesen: Zwar habe sich seit Elias Canettis ${ }^{288}$ Beschreibung des Waldes als der Deutschen Mentalitätsgeographie und des Tropenwaldes als dessen Antithese diese Opposition in ihrer Radikalität aufgelöst. Aber der Wald bleibe dennoch Ort einer „symbolüberladenen und moraldurchdrungenen Vorstellungswelt“, deren

\footnotetext{
${ }^{284}$ Flitner legt dar, wie Grzimek anhand seiner in Kein Platz für wilde Tiere geschilderten Reise im belgischen Kongo Bevölkerungsberechnungen anstellt, von der ,Heuschrecke Mensch“ spricht und Menschen als Sintflut für Tiere imaginiert. Vgl. Flitner: Vom ,Platz an der Sonne', S. 246 f. sowie Grzimek, Bernhard: Kein Platz für wilde Tiere. München 1954.

${ }^{285}$ Neben Elektrizität und Anbindung an das Funknetz versucht Sommer z. B. auch, eine touristisch orientierte Kunsthandwerksindustrie vor Ort anzustoßen, die wenig mit einheimischen Traditionen, dafür umso mehr mit einer globalisierten Vorstellung von afrikanischen „Handwerksprodukten" (SP, S. 177) zu tun hat.

${ }^{286}$ Programmatisch zu finden in den Ausführungen SP, S. 179 ff. Sommer beginnt hier mit einer kritischen Analyse der Entwicklungshilfeindustrie, gelangt aber rasch mit seinen ,weltläufig geschulten Einsichten“ (S. 180) zu einem verallgemeinernden Kahlschlagargument: „Wer Afrika wirklich helfen will, dürfte deshalb überhaupt keine Gelder geben. Wer Hilfe braucht und Hilfe haben will, muß sie sich verdienen, sonst bleibt Hilflosigkeit ein Dauerzustand“ (S. 180).

${ }^{287}$ So wenn er über ,den deutschen Mann“ schreibt: „Wenn der deutsche Mann, und speziell der Nordhesse, seinem Bier zusagt, neigt er zur innerlichen Aufweichung gegenüber seinem Artgenossen. Man wird rührselig, bevor bei terminalem klarem Korn kaum kaschierter Größenwahn aufkeimt. Früher wurden Kriege in dieser Laune gewonnen, doch mittlerweile tendiert man in Bierseligkeit zu praktischeren Visionen. Der deutsche Mann hat überdies die Eigenart, daß selbst leichtfertig gemachte Versprechungen verbindlich sind“ (SP, S. 123).

${ }^{288}$ Canetti, Elias: Massensymbole der Nationen. In: Ders.: Masse und Macht. München 2011 (Werke, Bd. 3), S. 197-209, hier S. 202 f.
} 
Bilder und kollektiv geteilte Vorstellungen ,,zumindest punktuell für den deutschsprachigen Kulturraum spezifisch“ 289 seien.

\subsubsection{Praxis: Fernfeldforschung}

Die den Text prägenden Anklänge an den Afrikareisebericht drohen teilweise das Erzählen von den Affen vor Ort und von der Arbeit der Feldforschung zu überlagern. Doch es finden sich in Sommers Schimpansenland auch etliche Passagen, die mit großer Detailfreude das wissenschaftliche Forschen vor Ort erläutern. Auch dies kann man als Anknüpfung an die Darstellungen in den Forschungsreiseberichten älterer Afrikareisender betrachten, die häufig naturkundliche oder ethnographische Schilderungen beinhalten. Diese Passagen lassen sich jedoch auch mit der These lesen, dass die veränderten Methoden der Affenforschung die Textstrategien, die Sommer für seine Darstellung der Zeit in Gashaka wählt, zumindest mit bedingen. Dazu lohnt sich ein Blick auf die geschilderten Forschungspraktiken und die Art und Weise, wie diese in Schimpansenland dem Laienpublikum, an das sich die Veröffentlichung richtet, erläutert werden: etwa wenn es um die räumliche Verteilung und materielle Beschaffenheit von verlassenen Schimpansennestern, die Konsistenz und den Wert fäkaler Hinterlassenschaften, die Erhebung biogeographischer Daten oder die Frage des Werkzeuggebrauchs und die Berechnungen über die Verteilung von Insektenarten im Gashaka-Wald geht.

\section{Nestervermessen}

„Nester haben einen entscheidenden Vorteil: Im Unterschied zu ihren Erbauern rennen sie nicht weg“ (31), so begründet Volker Sommer das Studium der verlassenen Schlafnester der Gashaka-Schimpansen. Weil es langwierig ist, Schimpansen an „neugierige Beobachter“ (31) zu gewöhnen, müsse, „[w]er deshalb vorhat, Schimpansen im Freiland zu erforschen [...] [,] sich zunächst mit ihren Hinterlassenschaften begnügen“ (31). Das Rüstzeug hierzu sind „Kompaß, Winkel- und Entfernungsmesser, Global Positioning System, Maßband und -schnur, Steckleiter, Gartenschere, Fernglas, Notizbrett“ (31). Mithilfe eines Londoner Doktoranden (Andrew Fowler), eines nigerianischen Studenten (Jeremiah Adanu) und seines Feldassistenten Hammaunde betreibt Sommer ,die Datenaufnahme an Schimpansennestern“ (32):

\footnotetext{
Der Nestbaum wird mit einem Plastiketikett numeriert, seine Koordinaten durch das Global Positioning System ermittelt. Das Gerät in der Größe eines Mobiltelefons in der Hand zu halten wird bald langweilig, weil das Kronendach die Signale der Satelliten oft blockiert oder ablenkt. Um Längen- und Breitengrad anzuzeigen, muß das GPS mit drei Satelliten verbunden sein. Aber bis sich der Empfang stabilisiert, das kann dauern. Andrew geht dazu über, das Gerät im afrikanischen Stil auf dem Kopf zu balancieren, während er andere Einträge vornimmt. (32)
}

\footnotetext{
${ }^{289}$ Flitner, Michael: Gibt es einen ,deutschen Tropenwald“? Anleitung zur Spurensuche. In: Ders. (Hg.): Der deutsche Tropenwald. Bilder, Mythen, Politik. Frankfurt a. M./New York 2000, S. 9-20, hier S. 13.
} 
Um die Leser/innen bei der recht technischen Beschreibung des Vorgehens bei Laune zu halten, streut so auch Sommer Anekdotisches ein, wie hier über das GPS-Gerät und Andrew Fowlers Umgang damit. Über mehrere Seiten wird von den Arbeiten der Nestdatensammlung berichtet - die jedoch in einem schimpansenlosen Wald stattzufinden scheinen. Dafür erfährt die Leserschaft von Schimpansenland viel über diesen Wald und seine Erfassung:

Im Durchschnitt beansprucht ein solcher Baum 65 Quadratmeter für sich. Auf einem Quadratkilometer wachsen dadurch $(1000 \times 1000 / 65=) 15391$ Stämme. Schimpansen können leicht Wohngebiete von 30 Quadratkilometern haben - was 461730 Bäumen entspräche. Da hätten wir viel zu tun. Um das zu vermeiden, vermessen wir eine repräsentative Auswahl, die wir durch die Transekt-Methode erhalten. Dabei werden Daten entlang einer gedachten geraden Linie durchs Gelände aufgenommen. Das Transekt soll lang genug sein, damit es durch sämtliche Vegetationstypen hindurchführt. Wir wählen dafür eine Linie von acht Kilometer Länge. Da wir ungefähr 1000 Bäume vermessen wollen, muß das Transekt - so finden wir durch Ausprobieren heraus - acht Meter breit sein. Die Fläche umfaßt damit $(8000 \times 8=) 64000$ Quadratmeter, das sind 6,4 Hektar oder ungefähr sechs Fußballfelder. Mit Kompaß, Hammer und Markierplättchen arbeiten Jerry und Andrew die Aufgabe nach und nach ab. (36 f.)

Die Gründlichkeit und Deutlichkeit, mit der hier das Verfahren bis hin zur Aufzeichnung der Rechnung dargestellt wird, setzt sich auch bei den anderen Forschungstätigkeiten fort.

\section{Kotsammeln}

Um der Nahrungszusammensetzung der Schimpansen nachzugehen, untersucht Andrew Fowler laut Sommer den Dung von Schimpansen und findet dabei ,zwei Dutzend kleiner grünlicher Päckchen aus Pflanzenmaterial““(162):

Am Nachmittag, zurück im Camp, wäscht Andrew mit einem Metallsieb den Dung im nahen Fluß aus. Zunächst wird Gras ausgewaschen, dessen Halme zu einem Knäuel verklumpt sind. Die „Päckchen“ aus Pflanzenmaterial entpuppen sich hingegen als genau 25 Blätter. Jedes Blatt ist einzeln im Kot verteilt. Auf bemerkenswerte Weise sind alle sorgfältig zusammengelegt. Andrew faltet das Laub auf. Bis auf einige durchsichtige Stellen ist es völlig intakt. Insbesondere weist kein Blatt Kauspuren auf. Daraus läßt sich schließen, daß Schimpansen die Blätter einzeln pflückten, sie dann falteten und unzerkaut schluckten. Seltsam. Andrew verstaut die Pflanzenreste in einer Herbariumspresse zum Trocknen. (162)

Es werden weitere Kotproben gesammelt und untersucht und es finden sich neben Blättern auch Würmer in ihnen. Sommer führt weiter aus:

Die getrockneten Pflanzenteile identifiziert Professor Emmanuel Obot von der Nigerian Conservation Foundation, einer der besten Botaniker des Landes. [...] In Röhrchen mit Alkohol finden die Würmer ihren Weg nach Japan in das Labor von Dr. Hideo Hasegawa an der Oita-Universität, der sie mikroskopisch untersucht. (162)

Wie genau das [pflanzliche Antiparasitikum] funktionieren könnte, erklärt uns Professor Michael Huffmann, ein Amerikaner, der in Japan lebt und als weltweite Koryphäre gilt für eine aufregende neue Forschungsrichtung mit einem geheimnisvollen Namen: ,Zoopharmakognosie‘. (163) 
Was bei Dian Fossey schlicht unter dem Stichwort ,dung washing ( $G M, 78)$ geführt wurde, wird bei Sommer als Prozess ausbuchstabiert, damit sich Leser/ innen genau vorstellen können, was Primatolog/innen im Feld machen und wie sie Schlüsse aus ihren Beobachtungen und Datensammlungen ziehen. Zudem führt Sommer genau an, welcher hochrangige Experte welche Art von Beitrag an der Ermittlung in dieser „Detektivgeschichte“ (161) geleistet hat.

\section{Biogeographische Datensammlung}

Um einen Überblick darüber zu bekommen, wie viele Schimpansen in der Gegend leben, wie viele gejagt werden, welchen Effekt dies auf die Populationen hat und wie stark Schimpansen überhaupt vom Aussterben bedroht sind, lässt Volker Sommer von einem seiner Promovenden Statistiken zu Affenauffangstationen auf dem afrikanischen Kontinent erstellen. Die Befunde gibt er wie folgt wieder: „Im Sommer des Jahres 2000 verzeichneten die Heime 474 Menschenaffen: 60 Gorillas, 10 Bonobos und 404 Schimpansen. Diese Zahl schließt jedoch weder jene eine, die in den Heimen verstarben, noch die vielen Säuglinge, die nie in einer Station landen“ (94). Mithilfe eines eigens entwickelten Fragebogens und einer Masterstudentin sollen daher weitere Daten zusammengetragen werden:

Wir nutzen die Bestandsaufnahme in den Heimen, um den Jagddruck auf wilde Bevölkerungen hochzurechnen. Dafür gehen wir davon aus, daß zwischen 1991 und 2003 insgesamt 75 Vellerosus-Schimpansen eingeliefert wurden: im Durchschnitt sechs pro Jahr. Das klingt gar nicht so schlimm. (97, Hervorheb. im Original)

In unsere Rechnung zur Bedrohung durch die Bejagung lassen wir deshalb eine simple Überlegung einfließen. Jäger schleichen sich in der Nacht an eine Nestgruppe an und schießen im Morgengrauen auf alles, was aus den Schlafstätten entkommen will. (98)

Weil von anderen Schimpansenpopulationen bekannt ist, dass ,,auf einen Säugling neun andere Gruppenmitglieder“ (98) kommen, überschlägt Sommer:

Jede Waise entspricht 50 aus der Wildnis entfernten Schimpansen. Die sechs pro Jahr in einem Heim landenden Vellerosus-Schimpansen repräsentieren somit 300 Menschenaffen; 270 davon wurden sofort getötet, 30 Waisen überlebten zumindest eine Zeitlang. Über einen Zeitraum von 13 Jahren hätte die Wildnis somit 3900 Menschenaffen verloren! (98 f., Hervorheb. im Original)

Nach dieser zuspitzenden Rechnung samt ihrer bedrohlichen Illustration (,,schleichen sich in der Nacht an“, ,schießen im Morgengrauen auf alles“) legt Sommer dar, wie man das natürliche Bevölkerungswachstum der Schimpansen anhand von Geburtsintervallen und Geschlechtsreife berechnen kann:

Das Modell muß weiterhin die maximale Bevölkerungsdichte der Schimpansen berücksichtigen, die im afrikaweiten Durchschnitt lediglich ungefähr 0,42 Tiere pro Quadratkilometer beträgt, womit also $(1 / 0,42=) 2,4$ Quadratkilometer nötig wären, um einen Schimpansen zu ernähren. Die Bevölkerung wächst am besten, wenn sie ungefähr 60 Prozent der Dichte erreicht hat, die das Biotop verkraften kann. In einem solchen 
Lebensraum beträgt der maximale Zuwachs mithin $(0,6 \times 0,42 \times 0,41=)$ 0,010 Tiere pro Quadratkilometer. Anders ausgedrückt: Pro Jahr kann unter günstigsten Bedingungen auf einer Fläche von 100 Quadratkilometern die Bevölkerung um einen Schimpansen zunehmen. Bei langsam reproduzierenden Arten wie Menschenaffen darf jährlich nur ein Fünftel der Tiere getötet werden, ohne daß die Bevölkerung abnimmt. Das wären $(0,010 \times 0,2=)$ 0,002 Schimpansen pro Quadratkilometer. Wiederum anders ausgedrückt: Pro 500 Quadratkilometer Lebensraum darf lediglich ein Tier entnommen werden, damit die Bevölkerung nicht sinkt. (99 f.)

Diese populationsökologischen Berechnungen zum Effekt der Jagd auf das Überleben der Art gehen derart noch einige Seiten weiter.

\title{
Insektenuntersuchungen
}

Auch die Überlegungen zu Insekten als Nahrung („Insektivorie“, 184) und dem dazugehörigen Werkzeuggebrauch von Schimpansen werden wieder anhand von Andrew Fowlers Erfahrungen eingeleitet (,Seine Bekehrung zur Primatologie begann für Andrew Fowler in der Garage“, 182). Im betreffenden Kapitel stehen nach anfänglichen Erläuterungen zur Bedeutung dieses Forschungsthemas für die Primatologie (184) vor allem Ameisen im Vordergrund:

\begin{abstract}
Für Schimpansen - und damit für unsere Forschung - sind vor allem die oberirdisch jagenden Treiberameisen interessant. [...] Soziale Insekten machen [...] ein Drittel der gesamten Tiermasse eines tropischen Urwalds aus. Für Treiberameisen allein läßt sich das folgendermaßen überschlagen: In einem gemischten Wald-Savanne-Biotop findet sich alle fünf Hektar eine Kolonie; das entspräche 550 Bauten in den ungefähr 26 Quadratkilometern des Wohngebietes der Gashaka-Kwano-Schimpansen. Diese Kolonien wiegen insgesamt $(40 \times 550=) 22000$ Kilogramm oder 22 Tonnen! Die ungefähr 35 Schimpansen der Kommunität bringen gemeinsam nur $(35$ x $40=) 1400$ Kilogramm oder 1,4 Tonnen auf die Waage, gerade mal 6 Prozent dessen, was die Ameisen wiegen. Umgekehrt ausgedrückt: Die Treiberameisen des Gashaka-Waldes wiegen 17mal mehr als die dort lebenden Menschenaffen. (186)
\end{abstract}

Diese Passagen führen dem Laienpublikum im Detail vor, wie die primatologisch Forschenden zu ihren Schlüssen über die Schimpansenpopulation vor Ort kommen: nicht nur anhand von Beobachtungen und dem Studium von Materialien, sondern auch anhand von abstrahierenden Überlegungen, wie vor allem die auf etlichen Hypothesen beruhenden Berechnungen zeigen.

Was, wie bereits angedeutet, an diesen Passagen des Weiteren auffällt, ist der Mangel an Affen. Zwar kann Volker Sommer darauf verweisen, dass sein Erfahrungs-Alter-Ego Andrew Fowler ,am 19. April 2001 auf Schimpansen“ (182) trifft und „zu seiner hellen Freude beobachten [kann], wie die Erwachsenen Zweige abbrechen, sie von Blättern befreien und die Stöckchen ins Loch eines Baumstammes einführen“(182). Aber Sommers eigener Sicht- und Beobachtungskontakt bleibt in Schimpansenland vage und selten. Es gibt eine Erstsichtung von einer Gruppe Schimpansen recht früh in seiner Erzählung und auch in der erzählten Zeit: 
Mir klopft das Herz. [...] Wir sind annähernd fünfzig Meter entfernt. Die Menschenaffen haben uns sogleich entdeckt. Das Sichtfeld meines Fernglases ist beinahe vollständig mit einem schwarzen Gesicht ausgefüllt. Eine Schimpansin mustert uns - ein wenig genervt vielleicht, doch ohne übertriebene Furcht. [...] Die Gashaka-Schimpansen sind größer, als ich sie mir vorgestellt hatte, und mit deutlichen Muskelpaketen unter metallisch schwarzer Haut herrlich schön. (21)

Nach dieser für Sommer beeindruckenden Erstsichtung, kommen Schimpansen zwar thematisch vor und werden beschrieben, statistisch erfasst und erörtert - aber es gibt kaum noch Sichtungen und schon gar keine ausführlichen Beobachtungsszenen. Einzelne Individuen und deren Lebensgeschichten fehlen ganz. Die handelnde Instanz, die in Sommers Darlegungen der praktischen Forschungsarbeit auftaucht, ist wie bei Fischer eine kollektive: Die Habituation und Beobachtung wird von einem Team ausgeführt, das Sommer zwar methodisch anleitet, und aus dessen Datensammlung er u. a. den vorliegenden Text schöpft. Aber an die Stelle der , ethnologischen“ Beschreibung der Schimpansengruppe und ihrer „Sitten und Gebräuche“" (s. o.) ist eine neue Art Feldforschung aus der Distanz getreten: Fernfeldforschung.

Schon Sommers Erstsichtung setzt $50 \mathrm{~m}$ und ein Fernglas zwischen ihn und das Gesicht der ,genervten“ Schimpansin. Das Wissen über Schimpansen, das Schimpansenland für sein Publikum aufbereitet, wird nicht am tierlichen Individuum oder im Kontakt mit der tierlichen Gruppe, sondern anhand von Materialien und Materie gewonnen: Hinterlassenschaften der organischen oder anorganischen Art. Nicht umsonst betont Sommer das ,[D]etektivische“ (198) der primatologischen Arbeit in Gashaka: Was er und sein Team praktizieren, ist die Deduktion schimpansischen Lebens anhand von Indizien. Das Bezeugen tierlichen Lebens und des interspezifischen Kontaktes ist hinter die handfesten ,wissenschaftlichen Beweise und die Überzeugungskraft der Statistik zurückgetreten. Ein Satz wie „Unser Herz schlägt bereits höher, wenn wir die Schimpansen lediglich hören“ (76), deutet darauf hin, wie rar das Erlebnis einer Sichtung ist. Selbst dort, wo Sommer vermeintlich auf ,eine Gruppe Schimpansen“ trifft, muss er konzedieren: „die ich nicht sehe, aber bei einem Bachlauf lärmen höre“ (104).

Die formale, inhaltliche und auch paratextuelle Verankerung von Schimpansenland im Abenteuer- und Afrikareisebericht scheint bei Sommer daher ebenfalls dem Ausbalancieren seines Textes zu dienen. Wie Fischer scheint er darum zu wissen, dass die Forschungsverfahren und -ergebnisse, das Affenwissen, für das Publikum durch ein empfindendes, erlebendes Subjekt dargeboten werden müssen. Der Ich-Erzähler als Forschungsreisender, welcher durch das Affenwissen von Schimpansenland führt, spielt die Rolle einer Vermittlungsinstanz und reichert den Bericht anekdotisch an. Dass Sommer dabei auf ein kulturelles Repertoire zurückgreift, das angelsächsische Autor/innen (und auch Julia Fischer) möglichst vermeiden, hat dreierlei Gründe: Erstens kann die unreflektierte Anleihe bei einem problematischen Genre in den Kontext einer noch immer vergleichsweise geringen Sichtbarkeit und sogar Abwehr der Aufarbeitung der deutschen Kolonialgeschichte und der damit einhergehenden Kolonialkultur in den deutschsprachigen Ländern 
gestellt werden. ${ }^{290}$ Zweitens zeigt sich dabei wie oben angedeutet die auch historische Problematik eines westlichen Naturschutzprogrammes, das wesentlich auf einem (post-)kolonialen Fundament beruht. ${ }^{291}$ Drittens schließlich stellt Sommer selbst die Verbindung zwischen der Affen- und Menschenbeobachtung her: Er nimmt wie erwähnt die klassische Pose des Anthropologen im Dorf ein und sieht strukturelle Parallelen in der ethnologischen und der primatologischen Feldbeobachtung, wenn er den Schimpansenforscher/innen in einer „Anfangsphase“ das Betreiben von „Ethnographie“ bescheinigt, nämlich das Berichten ,im Stile von Anthropologen, die mit einem unbekannten Volk leben, von den Sitten und Gebräuchen ihrer jeweiligen ,Ethnie““ (207). Mangels der Affen, die für das Publikum mit ethnologischen Anleihen aufbereitet beschrieben werden könnten, sucht sich Sommer das ,Abenteuer Afrika' um im Rahmen der gewählten Form des Forschungsberichts vergleichbar (von ,Afrika') berichten zu können. Die Form des Afrikareiseberichts antwortet damit auf eine Leerstelle, die sich in der und durch die Forschungspraxis ergeben hat.

\subsubsection{Affekte: Das Selbstgefühl von Zivilisierenden}

Durch die Rolle des Erzählers als Afrikareisender ist Schimpansenland stärker als Julia Fischers Affengesellschaft mit einem Subjekt belegt, das (sich) fühlt: Angesichts der Affen klopft Sommer das Herz (21). Er ist sich „des Privilegs bewußt, solchen wunderbaren Szenen beiwohnen zu dürfen“"(24), und ergriffen von „eine[r] Mischung aus Befriedigung und Glücksgefühl“ (25). Wie Fischer greift Sommer auch gern auf Affektphrasen zurück, die emotionale Reaktionen verlässlich anzeigen und durch ihre Floskelartigkeit wenig Raum für Bedeutsamkeit lassen. Wenn ein Löwe ums Zelt streicht, rutscht ihm „das Herz in die Hose“ (25); ein Leopardenruf „läßt einem das Mark in den Knochen gefrieren“ (35). Seine Frustration über die Regenzeit wird als „Hundstage“ (160) abgeheftet.

\footnotetext{
${ }^{290} \mathrm{Zu}$ dieser Problematik vgl. neben Friedrichsmeyer/Lennox/Zantop: Introduction z. B. Eckert, Andreas/Wirz, Albert: Wir nicht, die Anderen auch. Deutschland und der Kolonialismus. In: Sebastian Conrad/Shalini Randeria (Hg.): Jenseits des Eurozentrismus. Postkoloniale Perspektiven in den Geschichts- und Kulturwissenschaften. Frankfurt a. M./New York 2002, S. 372-392; Bürger, Christiane: Deutsche Kolonialgeschichte(n). Der Genozid in Namibia und die Geschichtsschreibung der DDR und BRD. Bielefeld 2017, S. 9 ff.; sowie bspw. die Debatte um das Berliner Humboldt-Forum, siehe Kuhn, Nicola/Peitz, Christiane: Raubkunst-Kritik am Humboldt-Forum: Horst Bredekamp: ,Die Werke sind nicht bezähmbar‘. In: Der Tagesspiegel vom 07.07.2015, Kultur, https:// www.tagesspiegel.de/kultur/raubkunst-kritik-am-humboldt-forum-horst-bredekamp-die-werkesind-nicht-bezaehmbar/12018870.html (15.05.2018); Zimmerer, Jürgen: Der Kolonialismus ist kein Spiel. In: Frankfurter Allgemeine Zeitung vom 09.08.2017, Feuilleton, S. 11.

${ }^{291}$ Dass Sommers historisches Bewusstsein allerdings auch in anderer Hinsicht auffällig wird, zeigt der saloppe Verweis auf die Shoah: Als er von einem befreundeten Zahnarzt ausgerechnet ausgedientes Zahngold als Spende für sein Forschungsprojekt erhält, lässt er dies bei der DEGUSSA einschmelzen, „der , deutschen Gold- und Silber-Scheideanstalt (Fußnote: Deren Schmelzöfen schluckten bereits die in Auschwitz angefallenen Zahngoldtonnen - was der Aktion einen gewissen makabren Charakter verleiht...)“ (SP, S. 125).
} 
Schimpansenlands Affekte sind aber überwiegend nicht affeninduziert oder allgemeiner tierinspiriert. Denn ob intendiert oder unintendiert, dieses Subjekt steht in der Tradition des Zivilisierenden. Diese Tradition bedingt ein bestimmtes Selbstgefühl, das auch den Erzähler in Schimpansenland ergreift. Im Vordergrund von Sommers Text steht eine Gefühlslage, die sich ebenfalls mit Jürgen Osterhammels Analyse der Zivilisierungsmissionen fassen lässt. Osterhammel beschreibt Zivilisierungsmissionen als konzeptuell auf Erfolg ausgerichtete Programme, die daher auch zwangsläufig auf programmatische Probleme treffen:

Selten sind Zivilisierungsmissionen genau in der Weise verwirklicht worden, wie Strategen und Visionäre es sich vorstellten. Dies ergibt sich bereits aus der Natur des Vorhabens und der Art seiner typischen Ideologisierung. In Konzepten der Zivilisierungsmission sind Misserfolge in der Regel nicht vorgesehen. Widerstände werden selten vorausgeahnt, und noch seltener stellt man sich auf sie ein. Die Unfähigkeit oder fehlende Bereitschaft der Objekte, sich zivilisieren zu lassen, übersteigt das Fassungsvermögen einer von sich selbst überzeugten Zivilisation. ${ }^{292}$

Als Reaktion auf Probleme der Implementierung zivilisatorischer Programme (Missionierung, Modernisierung, Entwicklungshilfe, Naturschutz, Demokratisierung etc. ${ }^{293}$ ) stellen eben diese Programme

bestenfalls die Idee der Undankbarkeit zur Verfügung. Es ist für das Selbstgefühl von Zivilisierenden von konstitutiver Bedeutung, sich als großzügig Gebende zu verstehen. Der Zivilisierer ist davon überzeugt, generös zu sein. Er spendet alles Mögliche [...]. Dafür nimmt er sich, was er für den gerechtfertigten Preis hält; vor allem erwartet er Dankbarkeit. Widerstand und Verweigerung werden als vermeintlicher Bruch dieser reziproken Spielregeln mit Erbitterung registriert und hart geahndet. ${ }^{294}$

Teil der Erbitterung kann die Übernahme eines kulturellen Ressentiments sein, wie es in Sommers ,Land und Leute'-Passagen sowie dem Mentalitätsdiskurs auftaucht, in dem jene ,Abgrenzung qua Abwertung' stattfindet, wie sie Reinhard Olschanski in seiner Kulturgeschichte des Ressentiments in Europa für eben dieses definiert: ein ,reaktive[r] Affekt zur Herabsetzung Anderer“, ${ }^{295}$ der im Projekt des zivilisatorischen Kolonialismus wirkt, den Fortschrittsdiskurs der Aufklärung im Sinne kolonialer Herrschaft umfassend funktionalisiert und weit über das Ende der kolonialen Epoche hinaus mentale Karten prägt, auf denen sich neue ,zivili-

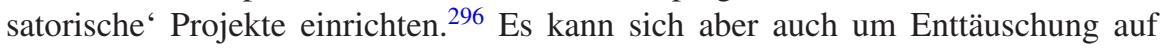
individueller Ebene handeln, um den Eindruck mangelnder Anerkennung für die eigenen Leistungen im Projekt der Zivilisierung:

\footnotetext{
${ }^{292}$ Osterhammel: Zivilisierungsmission und Moderne, S. 371.

${ }^{293} \mathrm{Zu}$ neuen Formen der Zivilisierungsmission siehe Osterhammel: Zivilisierungsmission und Moderne, S. 422.

${ }^{294}$ Osterhammel: Zivilisierungsmission und Moderne, S. 371.

${ }^{295}$ Olschanski, Reinhard: Ressentiment: Über die Vergiftung des europäischen Geistes. Paderborn 2015, S. 15.

${ }^{296}$ Olschanski: Ressentiment, S. $112 \mathrm{ff}$.
} 
Der unmittelbare Agent der Zivilisierung vor Ort [...] steht unablässig in der Gefahr einer doppelten Enttäuschung: einerseits durch die störrischen Objekte seiner Bemühungen, andererseits aber auch durch Politik und Öffentlichkeit im jeweiligen Mutterland, die immer wieder dazu neigen, seine Anstrengungen nicht hinreichend zu beachten, zu honorieren und zu finanzieren. ${ }^{297}$

Schimpansenland erzählt vordergründig eine Erfolgsgeschichte von der Gründung eines Forschungscamps und dessen ,zivilisatorischem" Ausbau, von den Bemühungen, den Naturschutz in den lokalen Strukturen zu verankern und auch für die Zukunft zu stärken, den Errungenschaften, die das Camp für die Gegend und die afrikanischen Gemeinden bedeutet, und den erfolgreich eingeworbenen Spenden. Sommer setzt sich nicht nur als Erzähler in den Mittelpunkt dieser Arbeit. Zwar verweist er ebenso wie Fischer auf seine Mitarbeitenden und formt seine Erzählung aus deren Arbeit mit den Affen und ihren Hinterlassenschaften. Aber Sommer schreibt: „Meine Investition in eine neue Generation nigerianischer Naturschützer ist auf dem besten Wege, Früchte zu tragen“ (213, Hervorheb. MS); ,Ich bin stolz darauf, die Markierung des Parkverlaufs konzipiert und vorangetrieben zu haben" (211, Hervorheb. MS). So sehr die Primatologie eine kollektive, interdisziplinäre und zunehmend materialtechnische Angelegenheit geworden ist, braucht sie bei Sommer einen ,Macher' wie ihn, der sie initiiert, organisiert und finanziert. In einem abschließenden Kapitel im Dienste des Naturschutzes („Im Anthropozoikum“, 218 ff.) stellt Sommer einen Vergleich des Starrsinns, den Naturschutz im 21. Jahrhundert braucht, mit demjenigen des Protagonisten aus Albert Camus' La peste an, indem er sich qua Emotionsschau selbst heroisiert:

Zuerst, ganz aus dem Bauch heraus, ist da eine Art Trotz, ein wenig wie jene Starrköpfigkeit, mit der Albert Camus die Hauptfigur seines Romans ,Die Pest' auszeichnet [...]. Denn genau das ist er laut Camus: ein Heroe. Er tut, was er für richtig und wichtig hält, ohne sich um Erfolg oder Mißerfolg zu kümmern. Er revoltiert selbst gegen das Unabwendbare. Ohne das Pathos heldenhaften Handelns bemühen zu wollen, stellt sich meine Situation ähnlich dar: Ich könnte nicht in den Spiegel sehen, würde ich nicht wenigstens versuchen, der Ausrottung einer der faszinierendsten Kreaturen auf Erden entgegenzuwirken. (224, Hervorheb. MS)

Dieses Selbstbild als starrsinniger Held im Dienste des Naturschutzes - und spezifischer: der Gashaka-Schimpansen - braucht jedoch auch die Widerstände, die Probleme und das Scheitern (der anderen), vor dem sich diese Art von Identität erst bilden kann. Sommer zählt davon genug auf und setzt ihnen etwas entgegen. Seine Klagen über die fruchtlosen Versuche, den Nationalpark als ,Raum für Tiere' zu sichern, gehen mit einer besonderen Zukunftsvision einher:

Vielmehr besteht vielleicht eines wie immer fernen Tages die Möglichkeit, ,leere' Waldgebiete effektiv zu erhalten - durch Elektrozäune, metertiefe Gräben, Blauhelmtruppen der UNO oder Satellitenaugen. (102, Hervorheb. MS)

\footnotetext{
${ }^{297}$ Osterhammel: Zivilisierungsmission und Moderne, S. $372 \mathrm{f}$.
} 
In nicht allzu ferner Zukunft, wenn vielleicht der Dschungel von Gashaka diesen Status [des Weltnaturerbes] erhält, könnten dort Blauhelme der UNO einmarschieren und zur Rettung einer Spezies beitragen. (226, Hervorheb. MS)

Sommers , utopische` Hoffnung liegt bei einer militärischen Lösung für den Naturschutz, die nicht nur das ethische Sentiment von Camus' Roman - Solidarität - zu pervertieren scheint, sondern eine bedenkliche Überwachungs- und Lagervision ist. ${ }^{298}$ Sie kann leicht als Neokolonialisierungsversuch gelesen werden. Sommers Primatologie ist zudem deutlich und affirmativ als eine westlich-weiße Angelegenheit ausgewiesen: „Denn als Ausländer müssen sie [die Freilandforscher/innen] keine falsche Rücksicht nehmen auf kulturelle, soziopolitische oder ökonomische Widerstände, die einen Einsatz oftmals rasch ausbremsen“ (217). Diese erwünschte Distanz zu den kulturellen, soziopolitischen und ökonomischen Gegebenheiten des Ortes, an dem das Projekt implementiert werden soll, diese Art von (auch affektiver) Nicht-Involvierung, zeigt sich auch an der Ambivalenz, die Sommer seinen einheimischen Mitarbeitenden entgegenbringt. In seiner Erzählung wird deren relative Bedeutungslosigkeit allein schon dadurch symbolisiert, dass der einzige nigerianische Doktorand, den Sommer erwähnt, zunächst durchweg aufgrund seines religiösen Eifers und seines Aussehens karikiert wird und dann kurz nach der Erwähnung seines Wertes für das Projekt verstirbt. Jerry ist zwar ,der erste afrikanische Student, den wir über die Stufen der Universitätsabschlüsse bis hin zur Doktorarbeit finanzieren. Ihn interessiert das Anliegen des Naturschutzes, und durch die Mitarbeit in unserem Projekt lernt er, auf frischen Wegen zu denken“ (212). Da Jerry überdies ,eine ehrliche Haut ist, natürliche Autorität besitzt, die von einer stattlich hochgewachsenen Statur untermalt wird, und alle möglichen Sprachen spricht, ist er der ideale Mittler zwischen Feldassistenten, Parkverwaltung, ausländischen Studenten und Forschern“ (212). Er ist damit Teil jener „Mittlerminorität“" ${ }^{299}$ die laut Osterhammel am anderen Ende der „Zivilisierungsbrücke“300 des Eliteprojektes Zivilisierungsmission steht. ${ }^{301}$ Als solcher löst er in Sommer Stolz auf die zunächst gelungen

\footnotetext{
${ }^{298}$ Dass Sommer mit einer solchen Idee nicht alleinsteht, zeigt ein Verweis Flitners auf verschiedene deutsche Politiker der 1990er Jahre, die Ähnliches bereits vorgeschlagen haben. Vgl. Flitner: Spurensuche, S. 12, 20.

${ }^{299}$ Osterhammel: Zivilisierungsmission und Moderne, S. 370.

${ }^{300}$ Osterhammel: Zivilisierungsmission und Moderne, S. 370.

${ }^{301}$ Jerry lässt sich mit Homi Bhabhas Hybriditätskonzept auch als Produkt jener ,kolonialen Mimikry“ lesen, unter der Bhabha das „desire for a reformed, recognizable Other, as a subject of a difference that is almost the same, but not quite" versteht. Bhabha: The Location of Culture, S. 124 f., Hervorheb. im Original. Wird das zu zivilisierende Subjekt zu sehr ,the same“, negiert es die abwertende Abstufung, auf der die koloniale Zivilisierungsmission basiert und macht diese nicht nur überflüssig, sondern bedroht auch die Identität ihrer Akteure und Akteurinnen. Koloniale Mimikry muss laut Bhabha somit immer beides sein, ,a complex strategy of reform, regulation and discipline, which ,appropriates " the Other" und „the sign of the inappropriate, [...] a difference or recalcitrance which coheres the dominant strategic function of colonial power". Sie braucht im Grunde das Ressentiment, um die Differenz dort wieder einkehren zu lassen, wo sie zu verschwinden scheint.
} 
scheinende Implementierung seines Programms aus. Bei einem Studienaufenthalt in England wird Jerry jedoch krank und verstirbt zurück in Nigeria an der - in Schimpansenland seltsamerweise namenlos bleibenden - Krankheit. ${ }^{302}$ Zwar schreibt Sommer anlässlich des als Anekdote dargebotenen Todes von „Schock, Traurigkeit, nachdenkliche[m] Schweigen", doch dies wird weniger als eigene Emotionen dargestellt, denn als ein allgemeiner Eindruck, wie der kurze Nachruf verdeutlicht: „Mit Jerry verliert unser Projekt viel: Freundschaft, Humor, Ratschlag, einen der immer mit anpackte“ (215). Jerrys Tod ist für Sommer vor allem eine Enttäuschung: Denn Jerry wird durch sein Sterben zu einer Investition, die sich nicht gelohnt hat. Jerrys Tod ist für Sommer noch knapp Anlass, für seine Leserschaft über die (erstaunlich vielen) Nigerianer nachzudenken, „die während unserer Projektarbeit plötzlich verstarben“ (214). Doch dies ist kein Versuch der einfühlenden Reflexion, gar der Solidarisierung mit der einheimischen Bevölkerung, sondern führt zu einer Bemerkung über polizeiliche Korruption und Aberglauben (215). Sommers Haltung ist die eines Rückzugs in die Resignation: „Daß eine Geschichte besser ausgeht, als sie beginnt, ist selten“ (214).

Die Ambivalenz eines ,Selbstgefühls von Zivilisierenden', das zwischen Stolz auf die Erfolge und Errungenschaften des/seines Projektes und Ressentiment und offener Enttäuschung über die Probleme und das Scheitern einiger Ansätze oder Investitionen (,,die bittere Einsicht“, 225) mäandert, zeigt sich letztlich auch in der Diskussion des Naturschutzanliegens selbst. Hier stellt Sommer zum Abschluss seines Buches die Überlegung an, ob sich Naturschutz überhaupt ,lohne', basiere das Konzept doch meist auf der ,irrige[n] Überzeugung, das freie Spiel der Natur würde paradiesische Zustände erzeugen“ (223). Der Einsicht „,[n]atürliche Prozesse sind gefühllose Vorgänge“ (222) stellt er jedoch die menschliche „Kapazität, Verlorengegangenem nachzutrauern, uns um Perspektiven zu sorgen“" (223), gegenüber, die „ein[en] emotionale[n] Gewinn“ (223) bringe. Das Selbstgefühl scheint dabei zu einem im obigen Zitat angeführten Selbstfühlen in der Rolle des Naturschützers als Helden zu tendieren, der sich gegen die Widrigkeiten der praktischen Umsetzung seiner Mission vor Ort und gegen Forderungen nach dem rational begründbaren Aufgeben einer solchen Mission stellt.

\footnotetext{
${ }^{302}$ Sommers Umgang mit Jerrys Erkrankung scheint durchweg von einem Misstrauen der einheimischen ,Arbeitsmoral ' gegenüber geprägt: Als Jerry in England über „Sehschwierigkeiten“ klagt, hält Sommer dieses „Wehwehchen“ für eine Ausrede, um vom Computerbildschirm fortzukommen und „mehr Zeit für eine Kirchengemeinde nigerianischen Ursprungs zu haben“ (SP, S. 213). Jerry will sich „,an Augenstar operieren lassen“, doch „,[d]as Augenlicht wird schwächer, die Beine versagen, selbst bis zur Latrine kann er nicht mehr allein“ (S. 213). Es gibt keine Diagnose, dafür aber bescheinigt Sommer dem Todkranken eine recht spezifische Verwahrlosung als ,Re-Afrikanisierung': „Weil seit Wochen niemand mehr seine Haare scherte, sprießen afrikanische Locken in alle Richtungen“ (S. 214). Letztlich übernimmt Sommer ironisch den Ratschluss der Angehörigen, es habe sich bei Jerrys Siechtum entweder um göttliches Schicksal oder um Hexerei gehandelt.
} 


\subsubsection{Die neuen Affekte der Forschenden}

Julia Fischers Affengesellschaft und Volker Sommers Schimpansenland sind stärker als populärwissenschaftliche Veröffentlichungen gestaltet als etwa Sarah Blaffer Hrdys The Langurs of Abu oder Barbara Smuts' Sex and Friendship in Baboons. Beide Texte blicken aber auch nicht im Sinne Jane Goodalls, Biruté Galdikas oder Robert Sapolskys als Memoiren auf das Leben als Feldforscher/in unter Affen zurück. Stattdessen berichten sie von Stand der Forschung zu Beginn des 21. Jahrhunderts. Zwar geht es hier am Rande auch um Forschung im Feld und die Etablierung von Forschungsstationen sowie natürlich auch um Affen. Doch ihr Anliegen ist die umfassende Wissensvermittlung und Aufklärung über Affenarten und den Sinn und Nutzen von Naturschutzbemühungen für ein Laienpublikum. Zu diesem Zweck wählen sie einen umgangssprachlichen saloppen Stil, der ihr Thema nahbar und eingänglich werden lässt, und werden dort, wo es um wissenschaftliche Überlegungen geht, ausführlich. Volker Sommer gestaltet seinen Text dabei im Modus eines Genres, welches als Medium der Vermittlung über ,exotisches“ Wissen tradiert ist. Gerade dadurch tritt die Qualität von Naturschutzprojekten als Zivilisierungsmissionen deutlicher hervor, als dies bei früheren Schriften der Fall war. ${ }^{303}$ Julia Fischer lässt dagegen eindrücklich werden, was die Professionalisierung der Primatologie für ihre Affektregime bedeutet: Der Verlust an individuellen, autobiographisch verwurzelten affeninduzierten Erfahrungen führt $\mathrm{zu}$ einer Klischierung emotionaler Eindrücke und zu Schwierigkeiten im Umgang mit dem als Vorbild dienenden Genre des Forschungsberichts bzw. der Forschungsmemoiren.

Beide Texte bilden die sich in den letzten Jahrzehnten verändernden Bedingungen der Feldforschung mit Affen ab: Die Techniken und Methoden ,im Feld“ haben sich ebenso verändert wie ,das Feld“ selbst. Das Schwinden der Affen sowie die zunehmende Erkenntnis über die Gefahr, die von Menschen für Affen ausgeht, führen zur Notwendigkeit, die Forschungsobjekte erneut aus der Ferne, teils sogar in ihrer Abwesenheit, zu erforschen. Dies verändert auch das Erzählen von der Forschung: Wo das Forschen immer kollektiver und kollaborativer wird, baut die Forschungsarbeit auf den Erfahrungen vieler auf. Diese heterogene Erfahrungsmenge kann nicht mehr im Modus der individuellen Memoiren sinnvoll wiedergegeben, Emotionalität nicht mehr im gleichen Maß als Teil von Forschungsprozessen exploriert werden. Einerseits nimmt Emotionalität in der Folge eine ,neue poetologische und stilistische Funktion ein: Sie legitimiert die Erzählinstanz als fühlendes Subjekt, das letztlich aber mittels anekdotischer Illustrationen den Mangel an eigenen emotionalen Erfahrungen zu überbrücken versuchen muss. Andererseits zeigt sich in der Analyse aber auch eine ,neue‘ Affektivität der Forschenden und ihre Bedeutung für dieses fühlende Subjekt. Bei Julia Fischer umfasst dies die Selbstwahrnehmung als Wissenschaftlerin mit Integrität und Pro-

\footnotetext{
${ }^{303}$ Eine Ausnahme bilden hier die bereits zitierten Passagen zum bedrohten Gorillahabitat aus Schallers The Year of the Gorilla, siehe Abschn. 3.4.3.
} 
fessionalität. Dort, wo dieses Selbstbild auch nur im Geringsten (bspw. durch einen Kleidungscode) bedroht wird, tritt spezifisch Scham als Emotion hervor. Volker Sommer dagegen beerbt seine Vorbilder auch in einem Selbstbild als Heros einer Zivilisierungsmission. Sein Selbstgefühl basiert auf Stolz auf das im Namen des Naturschutzes Erreichte und auf Enttäuschung bzw. Verbitterung, die die Tendenz zum Ressentiment beinhaltet. Beides sind Hinweise darauf, dass sich das Berichten und Darstellen von Affekten in der Forschung nicht nur unter dem Eindruck von wissenschaftsideologischen Programmen zum Umgang mit Emotionalität, sondern auch durch sich ändernde Methoden und Verfahren in der Forschungspraxis selbst verändern kann. Wissenschaftliche Affektregime können somit nicht nur hinsichtlich des Ausdrück- und Sagbaren untersucht werden, sondern auch dahingehend, was in ihnen überhaupt erfahrbar ist. Keine Affen bedeutet nicht, dass es keine Affekte gäbe. Ihr weitgehendes Fehlen in den Texten von der Feldforschung lässt jedoch andere Affektivitäten sichtbar werden, die in den zuvor untersuchten, älteren Texten nicht derart deutlich in Erscheinung getreten sind.

\subsection{In der Gegenwart: Fiktion als Verhandlungsraum einer Ethik des Emotionalen}

Wir forschen mit Affen, um etwas über sie herauszufinden? Welch fromme Lüge. Wir forschen mit Menschenaffen auf der Suche nach uns selbst. ${ }^{304}$

\subsubsection{Rekapitulation: Regime des Forschens}

Wie in den vorangegangenen Kapiteln gezeigt, unterliegen die Publikationen der Primatologie, ob als Forschungsmemoiren oder Forschungsberichte, unterschiedlichen externen Einflüssen und Bedingungen, die bei einer Beschäftigung mit den Affekten der Forschenden berücksichtigt werden müssen. Die Ansprüche eines Konzepts wissenschaftlicher Objektivität mit starken positivistischen Normen führen die beiden frühen, unterschiedlich forschenden und argumentierenden Primatologen Wolfgang Köhler und Solly Zuckerman in der ersten Hälfte des 20. Jahrhunderts zu einer Auseinandersetzung mit dem scheinbar unumgänglichen Anthropomorphismus-Vorwurf. Daraus resultieren aber auch jeweils eigene Textstrategien. Sie betreffen die Vereinbarkeit der eigenen Forschungserfahrung und -ergebnisse sowie des unausweichlichen epistemischen Anthropomorphismus mit den zeitgenössischen, durch wissenschaftliche Denkkollektive propagierten Normen der Darstellbarkeit von Forschungsemotionalität und Erkenntnis. Aber auch das Erzählen von der Forschung selbst unterliegt bei diesen Autoren derart strategischer Gestaltung. In den 1970er Jahren unterstützt eine neue Ideologie, in der sich Biologie

\footnotetext{
${ }^{304}$ Draesner, Ulrike: Sieben Sprünge vom Rand der Welt. München 2014, S.118. Im Folgenden, wenn zur Unterscheidung nötig, als SB geführt; Seitenangaben im Text.
} 
und Ökonomie außergewöhnlich produktiv vermengen und theoretisch ergänzen, die Implementierung eines neuen, die Primatologie auf lange Sicht prägenden soziobiologischen Denkmusters. Dieses formiert ein neues Tier-Konzept und verleiht einer zu diesem Zeitpunkt feministisch inspirierten Wissenschaft ein hilfreiches Instrumentarium, um dem Affekt-Gender-Konnex der Primatologie auszuweichen. Dabei lässt sich anhand der untersuchten Texte von Sarah Blaffer Hrdy und Barbara Smuts eine Verschiebung des Affektregimes nachvollziehen: Zunächst im Rahmen entemotionalisierender ethologischer Erklärungsmuster ,wegrationalisierte' Emotionen werden nach einer ,Neukalkulierung' im Einklang mit der Konsolidierung dieser Ideologie als neue Ressource für die Ethologie (und darüber hinaus) bei Tier und Mensch entdeckt und thematisiert.

Die kulturtheoretisch begründete Ironiefeindlichkeit eines historischen Moments in Verbindung mit dem Epochenbruch provoziert wiederum um den letzten Jahrtausendwechsel beim literaturversierten Robert Sapolsky eine humoristische Textstrategie, die Ironisierungen verwendet, um im Rahmen einer neu entstehenden Gefühlskultur $^{305}$,Immunität ‘ für die Thematisierung von Emotionalität im Feld zu gewinnen. Zugleich aber ermöglicht diese Strategie Kritik und Reflexion und fördert darin ein altbe-, aber zeitweilig verkanntes Potenzial der Ironie als Ethos zutage. Die sich verändernden ökologischen und methodischen Bedingungen der Forschungspraxis machen schließlich eine andere Gestaltung des erzählenden, fühlenden Subjekts der Forschungsmemoiren und den Rückgriff auf Textelemente und -strategien nötig, die neuere Texte von ihren Vorgängern auffällig unterscheiden und ihrerseits Aufschluss über den Stand primatologischer Forschung im 21. Jahrhundert liefern. Diese Texte zeigen dabei zugleich aber auch eine neue Qualität der Affekte der Forschenden als Authentizitätsbeleg für Protagonist/innen einer zunehmend kollektiven, interdisziplinären, materialtechnischen und eher affenfernen Primatologie. Zudem lassen die Veränderungen der Primatologie die Einbettung des Erzählens von der Forschung in kulturhistorisch bedingte, populäre Erzählformen in ihrer Funktionalität stärker hervortreten.

\subsubsection{Regiment des Gefühls}

Diese komplexe Verfasst- und Bedingtheit von Affektregimen der Primatologie ist dem wachsamen Auge der Literatur nicht entgangen. Die Popularisierung primatologischer Forschung durch die Forschungsmemoiren, wie sie durch Volker Sommer und Julia Fischer auch im deutschsprachigen Raum statt-

\footnotetext{
${ }^{305}$ Der Diskurs der ,Postironie‘ um 2000 scheint den Auftakt für eine neue Gefühlskultur als gefühlsgetriebene Kultur geliefert zu haben, die von der gegenwärtigen Zeitdiagnostik wahlweise als Zeitalter der Angst oder der Wut beurteilt wird, immer aber als Zeitalter einer neuen emotionalen Ernsthaftigkeit. Siehe bspw. Strasser, Johano: Gesellschaft in Angst. Zwischen Sicherheitswahn und Freiheit. Gütersloh 2013; Bude, Heinz: Gesellschaft der Angst. Hamburg 2014; Jensen, Uffa: Zornpolitik. Berlin 2017; Mishra, Pankaj: Age of Anger. A History of the Present, London/ New York 2018.
} 
findet, transportiert nicht nur die Affekte im Feld in die Belletristik. Sie provoziert im 21. Jahrhundert auch zunehmend eine Auseinandersetzung mit den Methoden, Grundlagen und Konsequenzen des Forschens im Rahmen von Literatur. Sind Affen bereits seit Langem Teil einer literarischen Aufmerksamkeit für die Grenzziehung des menschlichen am nicht-menschlichen Tier (vgl. Abschn. 1.3.2), und ist die Figur des/der einzelkämpferischen Forschenden etabliert (vgl. Abschn. 2.5.2 und 3.5), so treten in der Gegenwartsliteratur vermehrt der forschungspraktische Kontext der Primatologie, die ethischen Implikationen ihrer Ergebnisse und die Bedingungen und Konsequenzen ihrer Affektregime in den Vordergrund. In den USA erschienen in relativ kurzem Abstand Sara Gruens Ape House (2010), Benjamin Hales The Evolution of Bruno Littlemore (2011) und Karen Joy Fowlers We Are All Completely Beside Ourselves (2013). ${ }^{306}$ Ape House zeigt Gebärdensprachexperimente mit Bonobos im Labor und eine Art ,Sexploitation` des auffälligen Sozialverhaltens der Spezies in neuen Medienformaten. Im Bildungsroman Bruno Littlemore berichtet der gleichnamige schimpansische Protagonist in expliziter Anlehnung an Kafkas Rotpeter von seiner ,Menschwerdung' qua Liebesbeziehung zu einer Affenforscherin und Selbstbildung durch Shakespeares Dramen. ${ }^{307}$ In Fowlers We Are All Completely Beside Ourselves wird das emotionale Verhältnis der menschlichen Protagonistin zu ihrer Schwester elaboriert, die sich erst im Laufe des Romans als eine in einem Aufzuchtexperiment als Zwilling der Erzählerin erzogene Schimpansin herausstellt. $^{308}$

Im deutschsprachigen Raum sind 2014 gleich zwei Romane erschienen, die den Konnex von Emotionalität und Forschung betrachten: Ulrike Draesners Sieben Sprünge vom Rand der Welt und Bettina Suleimans Auswilderung. ${ }^{309}$ Beide Romane verhandeln ihre Kernthemen - generationsübergreifende Traumata und deutsch-polnische Vertreibungsgeschichte bei Draesner, die Desintegration einer Akademikerin auf der Suche nach der Befreiung der inneren Wildnis bei Suleiman - anhand von Figuren, die mit Affen arbeiten und forschen. Ähnlich wie bei Gruen, Hale und Fowler stehen dabei Ethiken des Umgangs mit dem Tier zur Diskussion. In Auswilderung geht es um eine großangelegte Studie mit Gorillas am „Deutsche[n] Institut für Anthropologie und Genetik“ (31) in Leipzig, in der zunächst evaluiert

\footnotetext{
${ }^{306}$ Gruen, Sara: Ape House. New York 2010; Hale: The Evolution of Bruno Littlemore; Fowler, Karen Joy: We Are All Completely Beside Ourselves. New York 2013.

${ }^{307}$ Siehe zu den auch emotionalen Implikationen dieser literarischen Darstellungen primatologischer Forschung: Richter: Ape Meets Primatologist.

${ }^{308}$ Durch den ,literarischen Kniff‘ dieser Spezies-Identitätsenthüllung und das emotionalisierende Potenzial ist der Roman vor allem für die Cultural Animal Studies und die normative Tierethik interessant. Siehe Calarco, Matthew: Boundary Issues. Human-Animal Relationships in Karen Joy Fowler's We Are All Completely beside Ourselves. In: MFS Modern Fiction Studies 60/3 (2014), S. 616-635; Gordon, Joan: Responsibilities of Kinship. The Amborg Gaze in Speculative Fictions About Apes. In: Extrapolation 57/3 (2016), S. 251-264.

${ }^{309}$ Suleiman, Bettina: Auswilderung. Berlin 2014. Im Folgenden, wenn zur Unterscheidung nötig, als $A W$ geführt; Seitenangaben im Text.
} 
werden soll, ob Gorillas Kriterien für den rechtlichen Personenstatus (im Roman: Personalität) erfüllen, wenn sie als Menschen erzogen und in Gebärdensprache geschult werden. Das negative Ergebnis wird für ein Auswilderungsprojekt genutzt, das die ,Subjects“ wieder dem Freiland und einem vermeintlich artgerechten Leben zuführen soll. In Sieben Sprünge hat der mittlerweile emeritierte Primatologe Eustachius Grolmann in seiner 40 Jahre währenden Karriere an der Münchner Universität mittels neurologischer Experimente an Schimpansen und Bonobos das Konzept des freien Willens widerlegt und hegt nun als Altersprojekt die Idee eines kommunalen Zusammenlebens von Bonobo-Kindern und Menschen-Alten. Seine Tochter Simone, ebenfalls Professorin der Primatologie in München, widmet sich dem Vergleich von Mensch und Affe und der ,conditio humana' des in Empathie fußenden epistemischen Anthropomorphismus.

Obwohl Affen somit als Versuchstiere eine Rolle spielen, stehen sie weniger als bei den Romanen Gruens, Hales oder Fowlers im Vordergrund. Das Augenmerk der beiden deutschen Romane liegt auf Forschungsethiken, die eher nach dem Verhältnis zur eigenen Emotionalität als nach dem Verhältnis zum Tier fragen: ${ }^{310}$ Was ist angemessene Affektivität in der Forschung? Wie viel ,Ich' bringt emotionales Verhalten in den Forschungsprozess? Was sind die affektiven Gründe für die Wahl einer Disziplin, die Konstitution und Affirmation eines Forschungsethos oder die Ausbildung eines Forschungsinteresses? Werden Haltungen zur Affenforschung durch bestimmte affektive Prägungen determiniert? Wie beeinflusst also Emotionalität im positiven wie im negativen Sinn die Forschungspraktiken und ihre Ergebnisse?

Beide Romane scheinen dabei, so eine vorläufige These, implizit oder explizit die historisch-kulturelle und/oder wissenschaftspolitische Produktion von Affektregimen aufzugreifen und verschiedene Haltungen zur Emotionalität in ihrer Konsequenz für die Forschungspraxis durchzuspielen. Folglich soll als Ethos im Folgenden eine Haltung verstanden werden, die zur Auseinandersetzung einlädt, ein „Ausdruck einer Selbstbeziehung, eines Sich-selbst-Positionierens als

\footnotetext{
${ }^{310}$ Dass es aber auch um Tierethik geht, steht außer Frage: Auswilderung ist durchdrungen von der Frage danach, was ,das Richtige " für die betroffenen Gorillas sei: Personenstatus oder Anerkennung als Spezies in ihrem eigenen Recht? Rückkehr in ihr gewohntes menschliches Zuhause oder ein Neuanfang mit, artgerechtem' Leben in der ,Wildnis'? Intellektuelle Überzeugung oder materieller Zwang? Entsprechend fiel das Augenmerk der Literaturkritik auch hauptsächlich auf diesen Aspekt, vgl. das Urteil „Moralische Bildung im besten Sinne“ bei Hayer, Björn: Im Paradies mit King Kong. In: Die Zeit vom 04.10.2014, http://www.zeit.de/kultur/literatur/2014-10/bettina-suleiman-auswilderung (03.01.2018). In Sieben Sprünge wird der aktivistische Furor des radikalen Tierschutzes ebenso angesprochen wie die (Un-)Zulässigkeit von invasiven Experimenten an Affen und Konzepte der artgerechten Haltung. Es dürfte auch kaum ein Zufall sein, dass recht früh in Sieben Sprünge auf eben jene Passage aus der Mailänder Ambrosiana-Bibel verwiesen wird, mit der Giorgio Agamben seine Überlegungen zum Tier-Mensch-Verhältnis in Das Offene eröffnet: die Darstellung des messianischen Gastmahls der Gerechten am letzten Tag der Menschheitsgeschichte, in der die Gerechten, das Fleisch des Leviathans und des Behemoth verspeisend, ,tierisches Anlitz“ (so die Formulierung in beiden Texten) tragen. Vgl. SB, S. 29; Agamben: Das Offene., S. $11 \mathrm{ff}$.
} 
der, der man ist und als der man handeln darf und soll“, 311 wie Martin Saar es im Anschluss an Michel Foucaults Beschäftigung mit der Geschichte ethischer Selbstpraktiken formuliert. Die beiden Romane können nun daraufhin untersucht werden, wie sie die obigen Fragen verhandeln und welche Rolle Emotionalität im Rahmen bestimmter abgebildeter Affektregime spielt, wie sich also die beiden Texte als Verhandlungsräume eine Ethik der Emotionalität in der Primatologie anbieten.

In Sieben Sprünge vom Rand der Welt präsentiert Draesner eine deutsch-polnische Familiengeschichte aus verschiedenen Perspektiven: Simone Grolmann, ihr Vater Eustachius, ihre Tochter Esther, die Großeltern Lili und Hannes Grolmann, der polnisch-deutsche Psychotherapeut Boris Nienalt, seine Mutter Halka und seine Tochter Jennifer bieten jeweils mit ihren eigenen Perspektiven Aufschluss über die Geschehnisse. In der Vergangenheit geht es um Kindheit und Jugend im Nationalsozialismus, um den Ersten und Zweiten Weltkrieg, die Flucht von Schlesien nach Bayern, das Nachkriegs(über)leben in Breslau für Halka und das Geflüchteten-Leben in Bayern für die Grolmanns. In der Gegenwart verfolgt Eustachius sein zunächst geheimes Bonoboprojekt und wird dabei in den Augen seiner Tochter immer verhaltensauffälliger. Simone versucht, sich dem verschlossenen Vater ergründend zu nähern und herauszufinden, warum er hoch verschuldet ist. Boris widmet sich der Erforschung der Traumata der Kriegskindergeneration und Simone und Boris verlieben sich über Boris' Arbeit mit Eustachius ineinander. In einer nahen Zukunft schließlich ist Esther erwachsen und trägt die Sorge für den greisen und sterbenden Eustachius, während Simone in Antwerpen ein Heim für Affen leitet. Der Prolog wird aus Sicht des Kleinkinds Eustachius erzählt und das letzte Kapitel gehört Emil, Eustachius' behindertem älteren Bruder, der auf der Flucht nach Bayern gestorben ist.

Während Sieben Sprünge derart mehrstimmig und komplex ist, bleibt Auswilderung einstimmig: Wir haben nur Marinas Ich, das uns von den Geschehnissen der Vergangenheit, der nahen und näheren Gegenwart erzählt. Die Erzählerin Marina berichtet autodiegetisch aus der Gegenwart des Auswilderungsprojektes, blendet aber immer wieder Analepsen auf die Anfänge ihrer Mitarbeit im Forschungsprojekt am DIAG ein. Auswilderung ist damit nah an diesem Forschungsprojekt und seinen Protagonist/innen. Da die Erzählerin jedoch, wie noch auszuführen sein wird, sehr um sich selbst kreist, ist die Glaubwürdigkeit des Wahrgenommenen nicht immer zweifelsfrei gegeben.

Eustachius, Simone und Marina sind die Figuren, die mit Affen forschen, und sie sind Figuren, an denen Emotionsdiskurse verhandelt werden. Die Romane arbeiten jeweils mit einer (oder mehreren) zugrunde liegenden und von den Figuren verhandelten These(n) zu Affekten: In Sieben Sprünge vom Rand der Welt ist

\footnotetext{
${ }^{311}$ Saar, Martin: Die Form des Lebens. Künste und Techniken des Selbst beim späten Foucault. In: Foucault, Michel: Ästhetik der Existenz. Schriften zur Lebenskunst. Hg. von Daniel Defert/ François Ewald. Frankfurt a. M. 2007, S. 321-343, hier S. 328; siehe auch Waldow, Stephanie: Schreiben als Begegnung mit dem Anderen. Zum Verhältnis von Ethik und Narration in philosophischen und literarischen Texten der Gegenwart. München 2013, S. 14.
} 
dies jene von der Übertragung von Emotionalität über Generationen hinweg, wie sie sich in der Forschung zu transgenerationalen Traumata findet: ,,[S]eelische Landschaften stempeln sich von einer Generation in die nächste hinüber" (14), wie Boris Nienalt in Sieben Sprünge feststellt. ${ }^{312}$ Auswilderung arbeitet dagegen mit der Vorstellung eines biologistischen Gefühlsdeterminismus: Empathie, so wiederholt hier die Erzählerin, sei weiblich und fehle Männern, zumindest jenen mit hohen Testosteronwerten. Beide Romane arbeiten diese Thesen anhand ihres Personals aus. In Sieben Sprünge betrifft dies vor allem die Figurenkonstellation von Eustachius und Simone, in Auswilderung die Erzählerin Marina und ihr Bedürfnis, sich in der akademischen Welt, in der sie sich bewegt, als Frau zu behaupten. Sie zeigen dabei, wie diese Figuren in historische, gesellschaftliche oder wissenschaftliche Affektregime eingebunden sind, die ihre Forschungsthemen ebenso bestimmen wie ihre Forschungsethik.

\subsubsection{Ulrike Draesners Sieben Sprünge vom Rand der Welt (2014)}

\subsubsection{Geheimgefühle}

Eustachius ,Stach“ Grolmann, „,international bekannter Neurologe und Hirnforscher, Koryphäe, Faktotum, Teil der aufblühenden Bundesrepublik, ängstlich im Gehirn, forsch nach außen, stur überall, ehrgeizig, geheimniskrämerisch“ (24), tritt mit 14 Jahren in den letzten Monaten des Zweiten Weltkrieges mit seiner Mutter Lilly und dem behinderten älteren Bruder Emil auf Geheiß der SS die Flucht aus dem schlesischen Oels in der Nähe Breslaus an. Auf dieser Flucht stirbt Emil. Im Laufe des Romans wird die Frage aufgeworfen, ob Stach seinen älteren Bruder getötet hat, weil dieser der SS beitreten wollte, nachdem die Familie ihn jahrelang unter größten Schwierigkeiten vor den nationalsozialistischen Euthanasieprogrammen beschützt hatte. Dies - die Fluchterfahrung und der Tod des Bruders - bilden das Trauma in Eustachius' Biographie. Nach der im Roman von Boris Nienalt formulierten These äußert sich dieses Trauma auch in der

\footnotetext{
${ }^{312}$ Boris greift hier ein psychotherapeutisches Forschungsfeld auf: Die zunächst im Kontext der Shoah-Aufarbeitung entstandene wissenschaftliche und therapeutische Beschäftigung mit ,vererbter" Traumatisierung wurde zunehmend auch auf andere historische oder individuell biographische Traumata übertragen, besonders im deutschen Kontext auch mit Hinblick auf die Nachkommen von Täter/innen. Vgl. Hardtmann, Gertrud: Children of Nazis: A Psychodynamic Perspective. In: Yael Danieli (Hg.): International Handbook of Multigenerational Legacies of Trauma. New York/London 1998, S. 85-95; Alberti, Bettina: Seelische Trümmer. Geboren in den 50er- und 60er-Jahren: Die Nachkriegsgeneration im Schatten des Kriegstraumas. München 2013; Rauwald, Marianne (Hg.): Vererbte Wunden. Transgenerationale Weitergabe traumatischer Erfahrungen. Weinheim 2013. Literatur nimmt dabei nicht nur im Rahmen therapeutischer Ansätze eine zentrale Rolle ein, vgl. Felman, Shoshana/Laub, Dori: Testimony. Crises of Witnessing in Literature, Psychoanalysis, and History. New York/London 1992; Schwab, Gabriele: Haunting Legacies. Violent Histories and Transgenerational Trauma. New York 2010.
} 
Weitergabe bestimmter Gefühle an die nächste Generation. ${ }^{313}$ Eustachius' Tochter Simone berichtet beispielsweise, der „Nachthimmel, egal, an welchem Ort, machte mir wenig Freude. Nachthimmel, den Laserstrahlen zerlegten, machte mir Angst“ (13). Auch hat sie „Angst vor Regen oder Schnee“ (13), ohne dass sie dafür einen Anlass in ihren eigenen Erlebnissen finden kann. Ihr ist bewusst: „Nun, wie erbärmlich, träumte ich die Alpträume eines anderen. Ein Stück kopiertes Leben im eigenen“ (19). Simone ist als Naturwissenschaftlerin, als „Biologin, Zellforscherin, Kennerin der Reaktionen von Natrium und Kalium, unerbittliche Gehirnstromreizerin und Gehirninterpretin“ (18), geradezu beschämt, dass Nienalts These auf sie zuzutreffen scheint, dass sie unter ,,mehreren geheimen Grundgefühlen“ leidet, „,die mich leiten und nicht sonderlich geheim“ (31) sind. Nienalt selbst, Sohn einer von Lemberg nach Breslau vertrieben Ostpolin und eines deutschen Breslauers, hat Angst vor abgedunkelten Räumen: „Rollos, Holzläden, zugenagelte Fenster machten mir Angst“ (82). Diese Angst geht auf Erfahrungen seiner Mutter Halka zurück, wie Boris erläutert: „Halka hatte im Sommer 1945 für Wochen im ersten Stock eines Breslauer Hauses hinter vernagelten Fenstern gelebt, zwischen Bränden, Leichen, Minen, Soldaten, Geflüchtete jeder Art, Repatriierten und Deutschen“ (83). Später ist aus Halkas Perspektive mehr über das zugrunde liegende Trauma zu erfahren: Halka erlebt die Vergewaltigung zweier ehemals deutscher Frauen im nun polnischen Breslau, entgeht nur knapp selbst der gleichen Gewalt, findet jedoch am selben Tag noch ein ihr (an-)vertrautes Kind verstümmelt im dunklen Hauseingang vor. ${ }^{314}$

Es geht jedoch um mehr als diese transgenerationalen Traumata, die als Vergangenheit den Damals-Betroffenen, so Eustachius' Enkelin Esther, „wie eine Pfeilspitze, knapp unter der Haut“ (505) stecken und die Nachgeborenen durch jene ,Geheimgefühle" heimsuchen. ${ }^{315}$ Nienalts Forschungsgebiet erstreckt sich auf die emotionalen Persönlichkeitsprofile derjenigen, die bei Kriegsende deutsche Jugendliche waren. Denn diese sind aufgewachsen in einer „Ideologieblase“,

\footnotetext{
${ }^{313}$ Vgl. zu dieser These in der psychologischen Forschung auch Radebold, Hartmut/Bohleber, Werner/Zinnecker, Jürgen (Hg.): Transgenerationale Weitergabe kriegsbelasteter Kindheiten. Interdisziplinäre Studien zur Nachhaltigkeit historischer Erfahrungen über vier Generationen. Weinheim 2008.

${ }^{314} \mathrm{Vgl}$. SB, S. $473 \mathrm{ff}$. In Halkas Worten wird der affektive Ursprung für das Trauma der Jalousie folgendermaßen umschrieben: „Ich sah nur, erstaunlich genug, eine Jalousie, obwohl vor dem Milchglas, das die obere Hälfte der Tür füllte, keine Jalousie hing, weder vor noch nach diesem Abend, nur jetzt hing sie da und flatterte im Takt meines Schluckkrampfes. Ich war blind, ich war taub. [...] In der Wohnung wühlte ich im Dunkeln nach neuer Kleidung, ich wagte nicht, Licht anzuschalten in diesem unheimlichen Haus in der noch unheimlicheren Stadt, die nicht einmal mehr die riesigen Brände erhellten. [...] Es war dunkel, ich sah dennoch deutlich. Keine Jalousie erschien, um mich zu retten".

${ }^{315}$ Gabriele Schwab verwendet in ihrer Beschäftigung mit dem Phänomen ebenfalls den Begriff einer solchen Heimsuchung, einem „,transgenerational haunting“, denn ,[i]t is the children or descendants [...], who will be haunted by what is buried in this tomb, even if they do not know of its existence or contents and even if the history that produced the ghost is shrouded in silence". Schwab: Haunting Legacies, S. 4 f.
} 
einer „braun-blutige[n] Eihaut, auf der Innenseite mit Heroen- und Kitschfilmen bespielt, mit Mythen und Blutzollliedern beschmiert, mit Wir-Gefühl tapeziert“" (14). Ihre Kindheit war ein ,ununterbrochene[r] Propagandafackelmarsch, manipuliert, eingeseift, auf Vaterland, Opfer, Kampf gedrillt, in eine enge Blut- und Rassewelt gesperrt" (36). Die nationalsozialistische Ideologie wurde, so die These hier, ,,[ü]ber alle Kinder gestülpt“ (14). Sie war ,[i] Kinder[]“"(22), die anderes als die Erwachsenen nur die Welt des ,Dritten Reiches“ kannten, ,seien nicht erzogen, sondern im wörtlichen Sinne verzogen gewesen: an ihrer Psyche habe man gezogen. Ihnen den Rahmen verzogen, die Menschlichkeit“ (22). Auch Eustachius’ Vater Hannes spricht davon, wie „sehr man sie [die Kinder] zu biegen vermochte“ (386), als er davon berichtet, wie das Kind Eustachius auf die Zwangssterilisierung seines älteren Bruders Emil reagiert (,Ich finde das richtig.“, 386):

Er war elf Jahre alt und wusste Bescheid. [...] Sie hätten das in Biologie gelernt. [...] Eustachius, die Augen blau, die Haut glatt, so klar und hart, saß unbeirrt und überzeugt an unserem Tisch. Er kannte keinen Zweifel. Ich erschrak, es war der Augenblick, als ich am tiefsten erschrak. (386)

Das Affektregime des Nationalsozialismus hat in seiner Kombination aus der propagierten „Abwesenheit elementarer menschlicher Gefühle [...] [wie] Empathie, Mitgefühl und liebevolle Zuwendung “316 und der ideologischen Produktion eines streng spezifizierten Gemeinschaftsgefühls ${ }^{317}$ eine besondere Relation von Emotionalität und Ethik produziert.

Im Roman stellt Boris' Tochter Jennifer angesichts eines Fernsehauftritts des alten Eustachius fest: „Grolmann wirkte unmenschlich. Etwas zutiefst Gefühlloses ging von ihm aus“(415). Simone empfindet ihren Vater als „exakt, kühl, kalkuliert“ (11). Er gehört zu den „,bundesrepublikanischen Karrieristen der Jahrgänge 1920-1936“, die Boris Nienalt als ,,[s]elbstüberzeugt, empathielos“ (74) beschreibt. Empathie-Mangelerscheinungen stellt sogar Eustachius selbst an sich fest, wenn er eingestehen muss, dass er Menschen fremder als Affen findet:

Bis heute war mir rätselhaft, dass diese anderen, Körper voller unberechenbarer Möglichkeiten, ein Innenleben haben mussten wie ich: Ständig dachten sie etwas, schmiedeten Pläne, beobachteten sich und erfanden neue Rollen; ständig hofften, träumten, liebten sie. (179)

Das Innenleben der Affen dagegen ist ihm ,vergleichsweise klar und liebenswert" (179). Diese Empathieproblematik im Umgang mit Menschen (aber nicht mit Affen) und der zumindest äußere Anschein der Gefühlskälte wird in

\footnotetext{
${ }^{316}$ Alberti: Seelische Trümmer, S. 92.

${ }^{317}$ Zum Stellenwert von Emotionalität für die nationalsozialistische Lebensphilosophie (etwas in der affektiven Mobilisierung der Hitlerjugend) bei gleichzeitiger Unterdrückung emotionaler Bindungen in der Eltern-Kind-Beziehung vgl. Alberti: Seelische Trümmer, S. 88 ff.
} 
Sieben Sprünge in Verbindung mit emotionaler Reife gebracht. Die Kriegskindergeneration erscheint im Roman als zugleich der Kindheit früh beraubt und emotional nicht ausgereift. Das Kind in Eustachius schwindet 1945 bis zur Unkenntlichkeit, wie sein Vater festhält:

Hannes hatte sich in Oels von einem 14-jährigen Kind verabschiedet. Dieses Kind hatte den Dackel zum Metzger gebracht. War nur mehr als Dreiviertelkind vom Metzger zurückgekehrt. Sah auf dem Bahnhof von Görlitz, wie die SS auf Frauen und Kinder einprügelte. Halbes Kind. Warf vorsichtig sein HJ-Koppel weg. Seither war, nahm Hannes an, nur mehr eine sehr kleine Kindergestalt in Eustachius versteckt. (370)

Doch aus diesem Verlust der Kindheit resultiert keine erwachsene Emotionalität. Wie Boris Nienalt ausführt, haben diese Kinder ,den Zweiten Weltkrieg und Hitlers Zusammenbruch nicht als verantwortliche Erwachsene“ erlebt, sondern ,als wache, empfindliche Jugendliche. Deren Gehirne sich soeben in der ,Pubertät‘ genannten letzten Reifungsphase ihrer Entscheidungs- und sozialen Wertungsinstanzen befanden, gemeinhin als Moral, Ethik und Gewissen bekannt“ (72). Kinder, geprägt durch ein System der mythologisierten Stärke und Sentimentalisierung der nationalen Gemeinschaft bei gleichzeitiger Empathieverweigerung für die vermeintlich Schwachen, der Gefühllosigkeit und zugleich des schwärmerischen Fanatismus, sind nun also in ihrer Pubertät, in der sich diese Prägung zu einer Haltung hätte ausbilden müssen, konfrontiert worden mit dem Zusammenbruch eben dieses Wertesystems und der Umkehr ihrer moralischen Leitwerte. ${ }^{318}$ Nicht umsonst interessiert Eustachius, wie seine Mutter Lilly später berichtet, am Milgram-Experiment ${ }^{319}$ weitaus mehr die Reaktion der, Täter' auf die Auflösung des Experiments als die ,Täterwerdung':

Leider endeten die Berichte an dieser Stelle, sagte Eustachius trocken, zu den Reaktionen der im Versuch so leichthin zu Tätern Mutierten finde sich nichts, dabei wäre eben das nun das Entscheidende, sagte er, wie diese Menschen sich fühlten, so unversehens zu Verbrechern geworden, und auch noch öffentlich. (430)

Durch diese ideologische (Ver-)Störung in der neurophysiologisch entscheidenden Phase der Pubertät, sei es, so Nienalts Einschätzung, Eustachius nicht möglich, ,seine Gefühle auf erwachsene Weise auszudrücken“ (142). Eustachius ist sich dieser „déformation Nazi“ (139), wie er es an einer Stelle ironisch

\footnotetext{
${ }^{318} \mathrm{Zu}$ den Kontinuitäten zwischen NS- und Nachkriegszeit gehört allerdings die „Verdrängung und Verleugnung von Gefühlen und Bedürfnissen“ (Alberti: Seelische Trümmer, S. 100) und ,the absence of any kind of emotional engagement at both the personal and collective levels" mit den historischen Fakten, wie Schwab anmerkt. Schwab: Haunting Legacies, S. 11.

${ }^{319}$ Vgl. Milgram, Stanley: Behavioral Study of Obedience. In: Journal of Abnormal and Social Psychology 67 (1963), S. 371-378.
} 
nennt, durchaus bewusst, sie ist schließlich trotz des großangelegten Programms der ,Entnazifizierung ' eine Volkskrankheit. ${ }^{320}$

Weitgehend unbewusst scheint für Eustachius jedoch der Einfluss des qua nationalsozialistischer Erziehung und ideologischen Werteumbruchs entstehenden Affektregimes auf sein Forschungsinteresse und -ethos. Informationen darüber erhalten wir nur aus der Perspektive der Anderen: „,Stärke', sagte mein Vater, ,Fühllosigkeit, Erfolg." Darum gehe es. Allemal im Beruf" (16), so gibt Simone Grolmann das Ethos ihres Vaters wieder. Doch bescheinigt sie ihm aus „Menschenfurcht“ mit Affen zu forschen: „Primaten, die große friedliche Utopie: ihre Ähnlichkeit mit uns, ihre Karamellaugen, ihr Schmelz“ (37). Eustachius' Anliegen als Primatologe war, ,an Affen etwas herausfinden über den freien Willen. Sie könne man nicht manipulieren, nicht wie uns. Nicht zum Töten von Artgenossen animieren“ (379), erinnert sich Hannes. Eustachius entlarvt schließlich den freien Willen als eine Fiktion, wie Simone zusammenfasst:

Endlich, nach Jahren, fand er die schützende Unfreiheit des Affen auch im Menschenkopf. Jedenfalls ihren Abglanz. Nicht der Wille des Subjektes bestimmte, was das Subjekt unternahm - sondern sein Gehirn. Seine Vernetzung und Taktung, seine Neigung, stets die gleichen elektrischen Zustandswolken zu bilden, gewohnte Simultanerregungen anderen Simultanerregungen vorzuziehen. Seine erlernte Gleichschaltung. (37)

Für Simone ist dieser Befund verbunden mit einem historisch und biographisch begründeten Anliegen Eustachius”: ,So rechtfertigte er sich - und andere“ (37).

\subsubsection{Die menschliche Schräglage}

Simone Grolmann betrachtet diese Forschungsarbeiten ihres Vaters also als Versuch, die eigene Teilnahme an einem ideologischen System aus den Mechanismen des Gehirnes heraus zu erklären: eine wissenschaftliche Ent-Schuldung des Kriegskindes. Aus den Ergebnissen dieser Forschung ihres Vaters zieht sie aber auch den wissenschaftlichen Beweis für die Affektthese Boris Nienalts: „Was du heute entscheidest, folgt stärker, als du annehmen möchtest, aus deiner neurologischen Geschichte. Woraus nicht philosophisch oder psychologisch, sondern

\footnotetext{
${ }^{320}$ Zum damit verbundenen Metaphernfeld siehe Hannes' Perspektive (SB, S. 365 ff.), wenn es in der Sicht der amerikanischen Militärbehörde auf die Nachkriegsdeutschen um Kontamination, Verseuchung, Infektion, Inflammation, um „Menschen mit verdorbenen, bösen Seelen“ geht. Auch die Traumaforschung greift dieses Metaphernfeld auf, um die Affizierung nachfolgender Generationen mit den Traumata der Ahnen abzubilden. So schreibt Gabriele Schwab über transgenerationale Traumata: „Violent histories generate psychic deformations passed on from generation to generation across the divide of victims and perpetrators. No one can completely escape the ravages of war or the dehumanizing effects of atrocities, not even those perpetrators who seem to have escaped unscathed or those who frantically rebuild their lives, their cities, and their nations. The damages of violent histories can hibernate in the unconscious, only to be transmitted to the next generation like an undetected disease". Schwab: Haunting Legacies, S. 3, Hervorheb. MS.
} 
physiologisch folgt: Was war, endet nicht“" (37). Sich selbst sieht sie nicht nur als Tochter, sondern auch als Wissenschaftlerin das Erbe ihres Vaters tragen: „Er hat 40 Jahre an Primaten geforscht. Bei ihm meinte Primaten: Menschenaffen. Ich forsche seit über 20 Jahren an Primaten: Affen und Menschen. Stach interessierte das Gehirn, mich interessieren Gehirn und Verhalten“ (12). Simone findet „den Affen im Menschen bemerkenswerter als den Menschen im Affen. Siehe Vater" (26). Ihre Arbeit baut auf der Forschung ebenso wie auf der Persönlichkeit ihres Vaters auf. Mit ihren Diplomand/innen beobachtet sie Gesten und Mimik von Schimpansen im Affenhaus in München als „,neueste[s] Projekt: Konzeption des Selbst beim Schimpansen“ (25); es geht ihr um „den Unterschied zwischen ihnen und uns" (35). Dabei entwickelt Simone in der Erzählzeit ihrer Perspektive eine Form der einfühlenden Forschung. Sie entdeckt, scheint es, ein Gefühl für die Forschung, das sie zu eher philosophischen als naturwissenschaftlichen Überlegungen führt:

\begin{abstract}
In den Genen finden wir eine Nähe, die wir nicht verstehen. Dabei fühlen wir, wie fremd sie sind: das Fell, der stark nach vorn gewölbte Oberkiefer, die tierische Hand, Klaue doch fast. Bis sich, mitten im Versuch, ein menschlicher Daumen auf dich zuschiebt. Zwar greift er anders als deiner, doch es ist die vertraute Form. Haarlose warme Haut. Du bist berührt. Spürst die Nähe. Da dreht der Affe den Arm. Du siehst Fingernägel, nicht Krallen. Doch wie grob die Nägel sind [...]. Du bist in einer Achterbahn: Jeder Blick zeigt dir Verwandtschaft und Differenz, und die Frage, wer diese Affen sind, verbindet sich, jetzt, endlich, denn du bist langsam und du hast dich dagegen gewehrt, mit der Frage nach dir selbst. (35)
\end{abstract}

Diese Erkundung der Gefühlsqualität der Verhaltensforschung als „vergleichende Menschenkunde“ (35) bringt ihr seitens ihrer eigenen Tochter, die Eustachius' Gefühlskälte geerbt zu haben scheint, den Vorwurf ein: „Sima verbonobot! [...] Sie verafft“ (107). Simone rede „nur mehr über Affengefühle“ (107). Tatsächlich aber, so stellt Boris fest, als er eine Vorlesung Simones besucht, beschäftigt sich Simone von einem epistemologischen Standpunkt aus mit der Rolle von Gefühlen in der Biologie des Verhaltens ebenso wie für die Erkenntnismöglichkeiten der Verhaltensforschung. So referiert Simone, man gehe heute davon aus, dass Menschenaffen keine kulturellen Systeme bildeten, weil es ihnen an „Vorstellungskraft in Bezug auf das Denk- und Gefühlsleben ihres Gegenübers“ (116) mangele. Sie wendet aber ein: „Sie sind empathiefähig, wenn auch in geringerem $\mathrm{Maß}$ als wir. Oder [...] anders empathiefähig" (116). Dass sie hier auch über ihren Vater Eustachius sprechen könnte, wird deutlich, wenn sie fortfährt: „Einige Forscher der älteren Generation halten Empathie für die wahre Folge des Sündenfalls. [...] Die Entstehung unserer Obsession, miteinander verbunden zu sein. Empathie, ein gehirnlicher Käfig, eine neurologische Zwangsjacke" (116). Wie in der aktuellen Diskussion um Anthropomorphismus als biologisch angelegtes Erkenntnissystem, ${ }^{321}$ sieht sie Empathie als „Human bias“, als „,[m]enschliche Schräglage“

\footnotetext{
${ }^{321}$ Vgl. Steiner: Anthropozentrismus, S. 29; Wild: Anthropomorphismus; Sober: Comparative Psychology. Siehe auch Abschn. 4.1.1.
} 
und ,,[u]nser[en] Neigungswinkel“ (118). ${ }^{322}$ Sie selbst, so trägt Simone laut Boris vor, sei in die Befragung des Gefühls durch einen Feldaufenthalt eingeführt worden, der sie im Alter von 19 Jahren, als sie ihren Vater zu einer Forschungsstation in den Kongo begleitete, verändert habe. Über den Aufenthalt selbst erfahren wir nichts, doch: „Nach meiner Rückkehr erkannte ich in den Augen unserer Forschungsaffen Gefühle. Angst, Spiellaune, Zärtlichkeit, Schabernack. Aber auch Trauer" (117). Simone blickt jedoch auf diese affektive Hermeneutik kritisch; es handelt sich ihrer Ansicht nach um einen trügerischen Effekt ihres Menschseins: „Ich ging in die Falle der Empathie. Eine Gehirnfalle. Ich stellte mir die Affen als Menschen vor" (117). ${ }^{323}$ Ulrike Draesner scheint hier auch auf die Tradition der affektiv fundierten Anthropomorphisierung zu rekurrieren, indem sie Simone von der ,pathetic fallacy “ der Nähe sprechen lässt und auf Ludwig Wittgensteins „Löwensatz“ verweist: „dass wir den Löwen nicht verstehen könnten, selbst wenn er unsere Sprache spräche“ (25). ${ }^{324}$ Simone fasst dies als: „Mensch mit Menschenmaske im Gehirn“ (25), wendet aber ein, Affen seien eben doch keine Löwen. Sie beschreibt die Versuche der Forschung ,den humanen Faktor im Experiment auszuschalten, [...] die große Geistesdehnung, hinüber zum anderen. In seine Affenheit" (118). Dabei unterlaufe jedoch immer wieder der Fehler, den Affen - wie in der Soziobiologie Sarah Blaffer Hrdys (vgl. Abschn. 4.2.1) - dann gar nicht als Gefühlswesen zu denken. ${ }^{325}$

Simone entwickelt ein affektiv gelenktes Nachdenken über die intellektuellen Voraussetzungen primatologischer Wissensgenerierung, das parallel zur Altersgefühligkeit ihres Vaters verläuft. Im Gegensatz zu dessen Emeritus-Forschung

\footnotetext{
${ }^{322}$ Die leitende Annahme der neueren Empathieforschung ist laut Breithaupt, ,dass Empathie nicht nur eine Eigenschaft oder Fähigkeit unter anderen ist, sondern wir als menschliche Wesen grundsätzlich von Empathie geprägt sind“. Empathie sei „zentraler Teil des Menschseins“. Breithaupt: Die dunklen Seiten der Empathie, S. 13 f. Siehe auch Abschn. 3.1.3.

${ }^{323}$ Auf die affektiv transformierende Funktion des Feldaufenthalts geht auch Eustachius ein. Als er Empathie und Mitgefühl bei Affen im Feld beobachtet, „hätte ich fast wirklich geweint, und der Atem stockte mir [...] aus Angst um mich: wie diese Nähe aushalten, dieses Mitgefühl, dieses so starke und unvermittelte" ( $S B$, S. 181). Der Feldaufenthalt bei den Affen bringt das ,Containment" in Gefahr, welches das Kriegskind Eustachius erlernt hat, um mit den Traumata der Vergangenheit umzugehen. Vgl. zu diesem ,Containment“ auch Alberti: Seelische Trümmer, S. $105 \mathrm{ff}$.

${ }^{324}$ Vgl. zum Sentimentalismus-Vorwurf des von John Ruskin geprägten Begriffs der ,pathetic fallacy ' für die Trope der Personifikation: Ruskin, John: Of the Pathetic Fallacy. In: Ders.: Modern Painters Volume III. Containing Part IV. On Many Things. London 1856, S. 157-172; Abrams, Meyer Howard/Harpham, Geoffrey Galt: A Glossary of Literary Terms. Boston ${ }^{9} 2008$, S. $241 \mathrm{f}$. Die Löwen-Bemerkung findet sich in Wittgenstein, Ludwig: Philosophische Untersuchungen. In: Ders.: Tractatus logico-philosophicus. Frankfurt a. M. 1999 (Werkausgabe, Bd. 1), S. 225-580, hier S. 568.

${ }^{325}$ Dabei vergesse man, so Simone, „die Affenwelt“, vergesse, ,[d] ass ein Gefühl, nicht ihr Trieb, ihr Verhalten bestimme: Angst vor dem anderen. Dass sie entscheiden konnten. Ein Stück Freiheit genossen. Dass sie auf Grund ihres auf sich und den anderen bezogenen Gefühls Gedanken entwickelten“ (SB, S. 120).
} 
wirft sie dabei ethische Fragen auf, die von epistemologischer Relevanz für die Primatologie der Gegenwart sind. Sie reiht sich aber als Figur damit auch ein in eine aus der Psychotherapie bekannte Beschäftigung der Kriegskinder-Nachfolgegeneration mit den Schwierigkeiten des Fühlens und der Befragung der ,pathologischen Normalität“ ${ }^{\text {326 }}$ ihrer Eltern. Doch in ihrer eigenen Familie hat immer noch die Vision des Vaters Vorrang. Während Esther ihrer Mutter empört vorwirft, sich mit einem Film auf der Website des Instituts ,zum Gespött aller Affenforscher“ (107) gemacht zu haben, weil sie „,[m]it Affen über den Boden gerollt und gelacht! Gelacht wie die Affen!“ (107) habe, sieht Esther in Eustachius' Alters-Projekt eine ,ureigene, lebensversöhnende Vision! [...] vollkommen anders als das Getue ihrer Mutter“ (108), wie Boris sie zitiert.

\subsubsection{Ethische Wenden des Emotionalen}

Eustachius Grolmann gibt sich mit der Existenzweise des Rentners nicht zufrieden, ist „entschlossen, nicht nur mitzuhalten, sondern noch einmal allen zuvorzukommen“ (159) mit seinem neuen Projekt, denn: „Meine besten Ideen hatte ich jetzt, jetzt den größten Lebensmut, die Lebenswut! Eine gänzlich neue Freiheit!“ (160). Befreit vom Anerkennungskarussel der wissenschaftlichen Gemeinschaft, in der Simones affektives Nachdenken und das Spiel mit den Affen bereits zum „Gespött“ dienen können, aber dennoch um die Anerkennung eben dieser Gemeinschaft bemüht, entwirft Eustachius die Vision eines Zusammenlebens von Affenkindern und Menschenalten und macht sich selbst dabei zum Untersuchungsobjekt. Er lässt auf seinem Grundstück ein unterirdisches Spielparadies für zwei illegal beschaffte Bonobos anlegen und sich selbst Elektroden einsetzen, die seine neuronalen Reaktionen auf das Zusammenleben mit den Affen abbildbar werden lassen. Er sieht darin einen von ihm einzuleitenden Paradigmenwechsel, der sich ebenfalls am , human factor' abarbeitet:

\footnotetext{
Wir mussten umdenken Richtung Interaktion, systematisch denken, das sprach sich endlich auch in der Affenforschung herum. Ich würde schneller sein, radikaler. An beiden Wurzeln ansetzen, Affe und Mensch. Wenn man sehen wollte, wie der human factor sich im Experiment auswirkte, wenn man die alte Erkenntnis umsetzen wollte, wirklich umsetzen, dass der Mensch sich aus keinem seiner Versuche, den er je mit der sogenannten Natur unternahm, herausrechnen ließ, weil exakt dort, in dieser Natur, sein blinder Fleck lag, dann musste man den Menschen, in diesem Fall mich, eben hineinrechnen. (160, Hervorheb. im Original)
}

Trotz des naturwissenschaftlich-experimentellen Tons, den Eustachius seinem Vorhaben verleiht, steht in dessen Zentrum ein affektives Anliegen: „,Affen für Alte' [...]. Alte Menschen sollten Menschenaffen bei sich zuhause aufnehmen. Zusammenleben, hätscheln, versorgen, spielen. Eine intensive, persönliche Form der Patenschaft [...]“" (186 f.). Es geht Eustachius um das Wohlergehen seiner Generation, deren emotionale Schwierigkeiten im Alter auszugleichen sind:

\footnotetext{
${ }^{326}$ Alberti: Seelische Trümmer, S. 73; auch S. 9 ff.
} 
Dank des Affen würden auch diese Menschen sich gebraucht fühlen. Eine Herzensfreude wären diese Fellwesen zudem, ein Menschenaffe eröffnet vollkommen andere Glücksund Unterhaltungstiefen als das herkömmliche Tier. Verwandte wirken nachhaltiger! (187)

Waren die Affen Eustachius zuvor Instrument, um die Entschuldung des ideologisch beeinflussten Tätervolks neurologisch zu betreiben, werden sie ihm nun eines, um das emotionale Leben der ehemaligen Kriegskinder und nun alternden Bundesrepublikaner/innen zu unterstützen. Dabei sind dem alten Eustachius die Affen nun Kind und ,mein Freund“ (164), „[m]ein Menschentier“ (177), „meine[] Fellkinder“ (178), „meine Jungbrunnen“ (177), „[m]ein Haustier“ (189), „[m]ein Lieblingstier“, „,meine[] urtümlichen Cousins“(191). Diese Hinwendung zum Emotionalen, das Propagieren eines ,[s]emanto-emotionale[n] Bonding[s]“ (179), ist im Roman wiederum konsequent Teil des von Boris Nienalt erforschten Phänomens. Denn Nienalt weist darauf hin, dass die durch die Biographie der Kriegskindergeneration verdrängten, begrabenen, verschütteten Gefühle im Alter wiederkämen. Simone sieht diese unerwartete Emotionalität ihres Vaters mit Beunruhigung, vielleicht sogar als Anzeichen einer beginnenden Demenz („Ich glaube, er wird weich.“, 11). In gewisser Weise zeugt die emotionale Wende tatsächlich von einer neuronalen Veränderung: „Das Gehirn über 70 werde durchlässiger, manches ordne es neu. Es produziere Direktheiten, geniale Rücksichtslosigkeiten. [...] ,Gefühle', sagte Nienalt, sind unsichtbare Menschen, sie gehen in deinem Haus umher“ (22). Diesem Bild der Emotionalität als etwas Weich-Wandelndes stimmt auch Simone zu, wenn sie eine neue Analogie für das Affektive verwendet:

Nach 20 Jahren Affen- und Menschenforschung stellte ich mir bisweilen vor, dass Menschen anders als ihre nächsten Verwandten innen aus Gel bestanden. Tiere waren verankert, eingebettet in ein sicheres Wissen davon, was man tat und was nicht. Im homo sapiens hingegen $s a ß$ an der Stelle des Instinktes etwas Weich-Zähes. Es ließ sich verformen. Als Wissenschaftlerin hätte ich gesagt: Das Gehirn ist ein distributives, der mesoskopischen Welt angepasstes, sich selbst organisierendes System nichtlinearer Dynamik. Sein Operationsraum ist nur endlich, endlich unmessbar, also menschlich unendlich. Das Innere, Mensch-Wabblige konnte man gelegentlich hören: Stöhnte es ,arrhh` oder ,grhh', wurde man krank; machte es Musik, war man verliebt. (239)

Das ,Weich-Zähe', ,Mensch-Wabblige' macht sich in Eustachius' Altersprozess immer weiter hörbar. Sein Altersprojekt scheitert, aber seine Hinwendung zum Emotionalen schreitet soweit voran, dass seine Enkelin Esther schließlich ,[m]it den Gefühlen“ (528) die Unterstützung ihres Freundes David benötigt, wenn sie ihren Großvater im Altersheim besucht. Auch Simone widmet sich in der Zukunft, von der wir durch Esther erfahren, ,neue[n] Studien zu Gefühlen und Spiegelungen [...], [der] Versorgung alter Primaten, ein[em] humane[n] Aspekt" (503). Sie greift also einen Gedanken ihres Vaters auf, der eine „,neue Humanität! Eine[] transgene[] Humanität! [...] Eine[] tragende[] Humanität“ (408) forderte, und kehrt ihn um - weg von der Instrumentalisierung des Affen für Forschung und emotionales Wohlergehen, hin zur Versorgung der Affen, die aus diesen beiden Nutzungsweisen des Tieres zurückbleiben. 


\subsubsection{Bettina Suleimans Auswilderung (2014)}

Bettina Suleiman setzt in ihrem Roman Auswilderung eine vergleichbare Frage an den Anfang ihrer Beschäftigung mit der Primatologie: Was geschieht mit den Affen, die in der Forschung für die Erkenntnisgewinnung benutzt werden, wenn sie ihren Zweck erfüllt haben? Und welches Recht hat die Forschung, darüber zu entscheiden, was für diese Affen ,das Beste“ sei? Suleiman bindet die Forschung in ihrem Roman an eine Institution, die als „Primatenforschungszentrum“ (25) des „Deutsche[n] Institut[s] für Anthropologie und Genetik“ (31) an das Wolfgang-Köhler-Primaten-Forschungszentrum des Max-Planck-Instituts für Evolutionäre Anthropologie in Leipzig erinnert. Durch diese institutionelle Identifikationsmöglichkeit wird nicht nur den gewichtigen tierethischen Fragen, die der Roman aufwirft, sondern auch der Forschungspraxis, die darin abgebildet wird, eine besondere ,realistische" Relevanz verliehen. Der Blick auf die tier- und forschungsethische Problematik sowie auf die Forschungspraxis der Primatologie ist jedoch in Auswilderung ausnahmslos durch die Perspektive der Erzählerin und Protagonistin Marina gestaltet. Diese Figur ist eine nicht ganz unproblematische. Sie scheint motiviert durch einen biologischen Gefühls- und Verhaltensdeterminismus, zerfressen von einem Gemisch aus Selbstzweifeln, Neid und Selbstbehauptungsdrang, und sie lässt ihr wissenschaftliches Verhältnis zu den Affen von ihren eigenen Bedürfnissen geleitet sein. Marina erscheint in der Folge nicht nur als verbitterte, sarkastische und selbstmitleidige, sondern auch als unzuverlässige Erzählerin. Denn ob das, was sie wahrnimmt, tatsächlich derart geschehen oder gemeint ist, steht desto mehr infrage, je weiter sie sich als Figur erschließt. Die Wahl einer solcherart problematischen Erzählerinnenfigur lässt sich aber verstehen als kritische Auseinandersetzung mit dem akademischen Betrieb und seinem Personal. Auswilderung, so die These, vermag mittels der Erzählperspektive dieser Figur auch danach zu fragen, wie die Primatologie und ihre Forschungsergebnisse durch epistemische Produktionsbedingungen und individuelle Persönlichkeiten bestimmt und zugleich interpretierbar werden.

Marina Heuter ist eine Mittdreißigerin, die seit ihrem 24. Lebensjahr am ,DIAG‘ in Leipzig arbeitet. Eingestellt wurde sie, wie sich im Laufe ihrer Erzählung herausstellt, im Herbst 1998 von Prof. Griffin Wilder für die Mitarbeit an einem Großprojekt, in dem experimentell über die Anerkennung des Personenstatus für Gorillas entschieden werden sollte. Mitte der 2000er Jahre, nach dem abschließenden negativen Befund zu dieser Forschungsfrage, schließt sich ein zweites Projekt an, in welchem die Auswilderung der Versuchstiere vorbereitet und schließlich durchgeführt werden soll. An diesem Projekt ist Marina nicht mehr beteiligt. Sie arbeitet aber dennoch - mittlerweile als Postdoktorandin - weiter mit einem eigenen Forschungsprojekt am selben Institut in Leipzig. In der erzählten Gegenwart, die sieben Jahre nach dem Ende des ersten Projektes liegt, wendet sich Griffin Wilder an Marina, da die Auswilderungsversuche kläglich gescheitert sind. Sie soll nun erneut in das Projekt eingebunden werden, um das Überleben der 
auszuwildernden Gorilla-,Subjects ‘ zu sichern. ${ }^{327}$ An dieser Stelle, an der Griffin Marina mit Video-Material von den gescheiterten Auswilderungsversuchen anzuwerben versucht, setzt Marinas Erzählung in Auswilderung ein.

Im Folgenden soll nun auf drei Verzerrungseffekte dieser Erzählperspektive eingegangen werden: Anhand von Marinas biologischem Determinismus (Abschn. 4.5.4.1), ihrer Performanz der Affektkultur des dargestellten akademischen Betriebs (Abschn. 4.5.4.2) und der affektiven Manipulationen (Abschn. 4.5.4.3), die sie vornimmt, zeigt sich die literarische Auseinandersetzung mit den epistemischen, affektiven und institutionellen Produktionsbedingungen der Primatologie in Auswilderung.

\subsubsection{Biologischer Determinismus}

Emotionalität ist für Marina als „,chemische Reaktion in meinem Hirn“ (49) „nichts, dem ich allzu viel Bedeutung beimessen musste“ (49), und doch ironischerweise von Bedeutung für alles, was die Protagonistin tut und als Erzählerin berichtet. Marina ist davon überzeugt, allein das biologische Geschlecht bzw. der dazugehörige Testosteronspiegel entscheide über die Befähigung zur Empathie. ${ }^{328}$ Sie hat diese Vorstellung so sehr verinnerlicht, dass sie sich selbst als Frau als einzig in der Lage sieht, aufgrund ihrer vermeintlich hormonellen Empathiebefähigung die ,richtige“ Lesart des Verhaltens anderer oder die ,richtige" moralische Entscheidung zu finden:

Ich hatte den niedrigen Testosteronspiegel, ich hatte die überdurchschnittliche Empathiefähigkeit. Kein Forschungsbericht dieser Welt hätte sagen können, wo die Subjects hingehörten. Ich wusste es einfach. (165)

Er [ein sudanesischer Koch] sagt es nicht, aber ich bin die mit dem außergewöhnlichen Einfühlungsvermögen und weiß, dass er mich merkwürdig findet, uns alle. (181)

Während Frauen in Marinas Perspektive intuitiv wissen, was die richtigen Entscheidungen sind, oder sich vollständig in andere hineinversetzen können, sind Männer ihrer Ansicht nach allein darauf aus, ihre Mitmenschen zu

\footnotetext{
${ }^{327}$ Im Roman wird die Bezeichnung ,Subjects` als englischer Begriff für Proband/innen herangezogen, weil ,Affen“ als ein zu besetzter Terminus erscheint: ,[W]ir verwendeten einen neutraleren Begriff - ,Subjects', Probanden -, der das Ergebnis unserer Arbeit nicht gedankenlos vorwegnahm" ( $A W$, S. 38). Auf die philosophischen Implikationen dieser Begriffswahl wird im Roman nicht explizit eingegangen, siehe hierzu aber Swiderski, Carla: Auswilderung. Eine experimentelle Vermessung von Affe und Mensch. In: Tierstudien 10 (2016), S. 80-89. Es ist dann eine Art ironische Fußnote, dass für den Erfolg der Auswilderung der Gorillas ohne Personalität, das „kognitive und emotionale Einverständnis der Versuchstiere in die Umsiedlung“, die Voraussetzung der ,explizite[n], verbalisierte[n] Befürwortung der Umsiedlung durch die Versuchstiere“ ( $A W$, S. 27), wichtig wird, also Kriterien, die nur ein rechtliches Subjekt gewährleisten kann.

${ }^{328}$ „Griffins Ringfinger etwa ist um einiges länger als sein Zeigefinger, was auf einen hohen Testosteronspiegel hinweist, unumgänglich verbunden mit geringer Empathiefähigkeit“ ( $A W, \mathrm{~S} .118$, Hervorheb. MS).
} 
dominieren, Raum zu besetzen und andere zu unterdrücken. In der Literaturkritik wurde dieses biologistische Programm der Erzählerin als konsequente Anwendung des ,evolutions- und kognitionsbiologischen Begriffsarsenals auch auf die Beschreibung der Forscherwelt“, 329 als „realistische[s] Wahrnehmungsraster einer Biologin, die überall genetisch programmierte Verhaltensmuster und Steinzeit-Männer sieht" ",330 bezeichnet. Das Problem mit diesem Interpretationsansatz ist nur: Marina ist keine Biologin. Sie ist eine Gebärdendolmetscherin (,ich bin aber auch nur Gebärdensprachdolmetscherin“, 236), deren Aufgabe im primatologischen Projekt darin besteht, die Kommunikation zwischen Mensch und Tier herzustellen. Marina wird zwar im Rahmen des Projektes zum Personenstatus der Gorillas promoviert, aber wie ihr anschließendes Postdoktorandinnenprojekt zu Babysprachgebärden („Mein Kreuzzug gegen das Baby Signing“, 16) zeigt, bleibt sie wissenschaftlich ihrer Disziplin, der Gebärdensprachforschung, treu.

Marinas Deutungsparadigma des biologisch determinierten Fühlens und Verhaltens scheint sich denn auch weniger auf den disziplinären Wissensstand der Verhaltensbiologie zu beziehen, als aus dem Fundus an (überwiegend nicht-fiktiver) Ratgeberliteratur zu speisen, die sie stetig konsultiert und zitiert: Psychologie für Führungskräfte (101, 102, 104, 153), Must-Haves für Ihre Karriere (69, 138, 162), Frauen im Management (17, 147, 152, 162, 172, 204), Sorge dich nicht, lebe! (162), Ethik für den Alltag (201), Erfolgreiche Erwachsene (201). Sie liest die Welt um sich herum und ihren eigenen Platz darin durch die Folie jener Populärbiologismen und -neurologismen, die im Rahmen eines solchen Arsenals von Technologien des Selbst ${ }^{331}$ zum Einsatz gebracht werden, um komplexe Verhaltensweisen komplexitätsreduziert zu begründen und häufig auch: bestehende Strukturen zu verstärken, etwa Geschlechterrollen festzuschreiben. ${ }^{332}$ Diese zwanghaft biologistische Hermeneutik führt in Auswilderung zum einen zu einer Konzentrationsschwäche der Protagonistin als Erzählerin. In Arbeitssituationen mit Männern gerät Marina beispielsweise häufig das Thema der Unterhaltung über den angelesenen Thesen zum räumlichen Dominanzverhalten und der vermeintlichen Notwendigkeit weiblicher Raumnahme im Dienste der Karriere aus dem Blick:

\footnotetext{
${ }^{329}$ Kämmerlings, Richard: Sind Gorillas vielleicht die besseren Menschen? In: Die Welt vom 15.11.2014, Literatur, https://www.welt.de/kultur/literarischewelt/article134369701/Sind-Gorillas-vielleicht-die-besseren-Menschen.html (03.01.2018).

${ }^{330}$ Kämmerlings: Sind Gorillas vielleicht die besseren Menschen?, o. S.

${ }^{331}$ „Technologien des Selbst“, so Michel Foucault, ermöglichen es dem oder der Einzelnen, ,aus eigener Kraft oder mithilfe anderer eine Reihe von Operationen an seinem Körper oder seiner Seele, seinem Denken, seinem Verhalten und seiner Existenzweise vorzunehmen, mit dem Ziel, sich so zu verändern, dass er einen gewissen Zustand des Glücks, der Reinheit, der Weisheit, der Vollkomenheit oder der Unsterblichkeit erlangt“. Foucault: Technologien des Selbst, S. 289.

${ }^{332} \mathrm{Vgl}$. Fine, Cordelia: Delusions of Gender. How Our Minds, Society, and Neurosexism Create Difference. New York/London 2010. Zum Zusammenhang von Ratgeberliteratur und Selbsttechniken im Neoliberalismus vgl. Illouz: Gefühle, S. 41 ff.; Kaindl, Christina: Frei sein, dabei sein. Siehe auch Abschn. 4.2.2.
} 
Damit er [Griffin] sich hinsetzt - im Stehen bin ich zwei Köpfe kleiner als er -, harre ich aus auf meinem Schreibtischstuhl, dessen Höhe man, anders als die meiner Besuchersessel, verstellen kann. Wenn es nach mir ginge, würde ich zwei oder drei Zentimeter auf ihn hinabblicken. Niemals justiere ich meinen Stuhl so hoch, dass die Leute es merken, es geht um die subliminale Wirkung. Nur einer von vielen hilfreichen Tipps aus dem Handbuch Frauen im Management: Nutzen Sie bei Verhandlungen im eigenen Büro Ihren Heimvorteil: Ihre Sitzhöhe verkörpert Ihre Position. [...] Da er keine Anstalten macht, auf meine Augenhöhe herunterzukommen, stemme ich mich jetzt doch aus dem Stuhl, mit beiden Armen. Eine Sekunde lang stehe ich ungeschützt im Raum. Dann ziehe ich mich ans Fenster zurück, zwei kleine Schritte, lehne mich an die kalte Heizung. Er blickt erwartungsvoll auf mich herab, immer noch, aber mit verringertem Gefälle. [...] Obwohl ich jetzt stehe und er sitzt, thront er immer noch über mir. (16ff., Hervorheb. im Original)

Erwartungsvoll schaut Griffin Wilder auf Martina herab, weil er sie zur Mitarbeit an seiner Studie überreden will. Aber Marina ist über drei Seiten hinweg zu sehr mit der Positionierung im Raum beschäftigt, als dass sie auf seine Argumente und Bemerkungen fachlich eingehen könnte. In einer ähnlichen Szene interpretiert sie eine Gesprächspause als Dominanzgeste und verfällt wieder in Territorialverhalten:

Die Pause ist als Gelegenheit für mich gedacht, mich bei ihm zu bedanken. Mich hinzusetzen, besser noch auf die Knie zu fallen, mich klein zu machen - aber warum? [...] Ich könnte mich beispielsweise auf seinen Schreibtisch pflanzen - endlich meine schweren Beine ausruhen und trotzdem größentechnisch dominieren. Aber wenn eine Frau so etwas macht, ist es eine Anmache. [...] Welches Territorium kann ich besetzen, wenn nicht seine Tischplatte? Noch ein Tipp aus dem Handbuch Frauen im Management: Nehmen Sie Raum ein - mit Ihrem Körper, Ihren Gesten. So schüchtern Sie Ihren Gegner ein. Ich beginne an den Buchreihen entlangzugehen, Griffins Besitz kritisch zu begutachten, ihn anzufassen, als wäre das mein gutes Recht. (152 f., Hervorheb. im Original)

Auch als Marina im Büro des Institutsdirektors Fischer das darin gehäufte materielle Arbeitspensum begutachtet - ,halbmeterhohe Papier-, Mappen-, Bücherund DVD-Stapel, im rechten Winkel zur Tischkante angeordnet, ein[] natürlicher Sichtschutz“ (209) -, deutet sie dieses Umfeld sogleich psychologisch und lässt ihr selbstverschriebenes Verhaltensprogramm anspringen:

Die Menge der von diesem Mann zu bewältigenden Arbeit, oder die Größe des von ihm besetzten Territoriums, sollen mir Ehrfurcht einflößen. Ich soll Angst bekommen, ihn zu stören. Nehmen Sie Raum ein - mit Ihrem Körper, Ihren Gesten. Ich rutsche in meinem Sessel noch tiefer, mache es mir bequem. Wenn er nicht auf Augenhöhe mit mir sein will, bitte sehr, kann er haben. Die Beine stelle ich so breit nebeneinander, es ist geradezu obzön. (210, Hervorheb. im Original)

In jeder dieser Passagen rekurriert Marina im Zitat auf die Ratgeberliteratur und kreiert erst durch die Kontextualisierung eine problematische Selbstwahrnehmung als Herabsetzung: Ihr Verhalten, ihre Position, ihr Stand dem Anderen gegenüber scheint mangelhaft und ist daher zu optimieren. Dies zeigt sich auch in Hinblick auf die Eigenschaften, die sie sich zuschreibt: Marinas zwanghafte Fokussierung auf ,Biologie‘ nimmt so zum anderen überwiegend (selbst-)abwertende Züge an, 
weil sie auf Stereotype zurückgreift und zugleich - indiziert durch die verinnerlichte Ratgeberliteratur - im Paradigma der Selbstoptimierung stattfindet. ${ }^{333}$ Auf ihren tätlichen Angriff ${ }^{334}$ auf einen Doktoranden Griffins zurückblickend heißt es von der Mittdreißigerin: „Ja, ich habe keine Ahnung, wie man kämpft, ja, ich bin nur ein Mädchen“" (210, Hervorheb. MS). Der Klang ihrer eigenen Stimme lässt sie bisweilen ,rot anlaufen, er ist weiblich, aber nicht im erotischen, sondern im traditionellen Sinne, schrill und hysterisch" (149, Hervorheb. MS). Um den offensichtlich empfundenen Mangel, Frau unter Männern zu sein, zu kompensieren, springt, wie schon dieser Angriff auf Arne Müller zeigt, Marinas Selbstbehauptungs- und Selbstverteidigungsreflex aggressiv auch dort an, wo dafür kein Anlass besteht. Als ihr Kollege John mit ihr ein freundschaftliches Gespräch darüber führt, was aus verhaltensbiologischer Sicht das ,beste Interesse ' für die Gorillas sei, und dabei eine andere Meinung vertritt, sieht Marina darin sogleich ,Mansplaining ‘:335 Sie gesteht ein, dass sie ,naiv klang in seinen Ohren“ (90), mit ihrer Behauptung, man müsse die Gorillas zu ihrem Glück zwingen,

[a]ber die Wahrheit ist unkompliziert, und wenn ein Mann das Gegenteil behauptet und dir jovial anbietet, sie dir zu erklären - oder es ungefragt tut, in den meisten Fällen, und dann auch noch erwartet, dass du dankbar bist dafür -, dann wehrst du dich besser beizeiten. So viel hatte ich begriffen. Ich war eine Frau, ja, aber ich war kein kleines Mädchen mehr. (90, Hervorheb. MS)

In einer anderen Situation überzeugt sie jemanden ,mit Hysterie wahrscheinlich, vor der Männer Respekt haben“ (239). Gerade in Situationen, in denen sie so gegen vermeintlich ,männliches 'Verhalten zu opponieren und sich als Frau im wissenschaftlichen Betrieb zu behaupten glaubt, schreibt sich Marina durch die Adaption eines Vulgärbiologismus in die Tradition eines misogynen Geschlechterdiskurses ein, der sie als Mängelwesen begreift. ${ }^{336}$ Dieser Diskurs scheint, so zeigt auch die Interpretation der Literaturkritik, in Zusammenhang mit der Primatologie

\footnotetext{
${ }^{333}$ Vgl. Mayer, Ralf/Thompson, Christiane: Inszenierung und Optimierung des Selbst. Eine Einführung. In: Ralf Mayer/Christiane Thompson/Michael Wimmer (Hg.): Inszenierung und Optimierung des Selbst. Zur Analyse gegenwärtiger Selbsttechnologien. Wiesbaden 2013, S. 7-28.

${ }^{334}$ Übermüdet und trotzig rammt Marina nach einer Art Zusammenbruch vor dem Gorillagehege Arne Müller den Kopf in den Magen, um den Gorillas, allen voran Yeh-teh, zu demonstrieren, dass sie nichts mit den ,[b]öse[n] Männer[n]“ ( $A W$, S. 140) des Projekts zu tun habe, sondern auf ihrer Seite stehe.

${ }^{335}$ Gemeinhin wird ,Mansplaining “ verstanden als ,explaining without regard to the fact that the explainee knows more than the explainer, often done by a man to a woman". Rothman, Lily: A Cultural History of Mansplaining. In: The Atlantic vom 01.11.2012, https://www.theatlantic.com/ sexes/archive/2012/11/a-cultural-history-of-mansplaining/264380/(03.01.2018). Vgl. auch Solnit, Rebecca: Men Explain Things to Me. Chicago 2014, S. 1-4.

${ }^{336}$ Zur Konstruktion der Frau als Mängelwesen vgl. Rohde-Dachser, Christa: Expedition in den dunklen Kontinent: Weiblichkeit im Diskurs der Psychoanalyse. Berlin u. a. 1991. Siehe auch Honegger: Die Ordnung der Geschlechter.
} 
als Verhaltenswissenschaft zu stehen, die im Menschen evolutionäres Verhalten ortet. Dabei wird durch die Erzählerinnenfigur jedoch deutlich durchgespielt, welche Konsequenzen eine unreflektierte Popularisierung naturwissenschaftlicher Theorien für die Handlungsoptionen eines Individuums und für dessen Verhalten im Arbeitsumfeld hat.

\subsubsection{Akademische Affektkultur}

Trotz Marinas Behauptung, Emotionalität müsse nicht allzu viel Bedeutung beigemessen werden, sind ihre ersten Erfahrungen im Personalitätsforschungsprojekt geradezu hyperbolisch affektiv. Bei der ersten Gorillabegegnung, die sie als Studentin im Primatenforschungszentrum erlebt, hat sie ,nachdem ich dem ersten Gorilla in die Augen geschaut hatte, ein Gefühl, wie wenn das Space Shuttle abhebt" (25). ${ }^{337}$ Danach kann sie sich nicht mehr vorstellen, ,die nächsten fünfundvierzig Jahre [...] mit Lehre und Erforschung solch atemberaubender Angelegenheiten wie Kontrastiver Syntax, Genderaspekten der Gebärdensprache und frühkindlichem Spracherwerb“ (25) zu verbringen. Bei der großangelegten ,Zugriffs'-Aktion, in der in einer Nacht alle Gorillas aus ihren menschlichen Pflegefamilien geholt werden, „ein freudiger Tag, für jeden Beteiligten“ (35), wie Wilder es nennt, schaut sich das versammelte Team in Live-Übertragung an, wie Polizeieinheiten und Pfleger mit Betäubungsgewehren die ,Subjects“ aus ihren Schlafzimmern holen, notfalls fesseln und narkotisieren. Bevor es losgeht, sind alle aufgeregt und Marina macht sich ,fast in die Hose vor Freude“ (35). Als Griffin ihr schmeichelt und sie auffordert, beim neuen Versuch der Auswilderung sieben Jahre später wieder dabei zu sein, heißt es schließlich: „,[M]ir [ist] nach Schreien und Jubeln zumute. Ich bin aufgeregt wie eine Braut kurz vor ihrer Entführung“ (28). Das Bild der Akademikerin als Braut, die sich mit der Wissenschaft vermählt, ist das einer säkularen Nonne. Es entspricht dem Affektregime, dass Marinas Blick auf die akademische Welt bestimmt. Durch den ersten, erweckungserlebnishaften Blick in Gorillaaugen geht sie eine scheinbar unauflösliche Bindung mit der Forschung ein, und diese wird mit dem beruflichen Eintritt in das Deutsche Institut für Anthropologie und Genetik als „Kathedrale der Wissenschaft“ (31) besiegelt. „Gott ist Prof. Dr. Griffin Wilder, Direktor der Vergleichenden Psychologie“ (31), behauptet Marina und definiert dadurch diese Beziehung als sakral. Dass sie diesen ,Gott' und Doktorvater auch unter erotischen Gesichtspunkten betrachten kann, macht das Verhältnis nicht weniger problematisch. ${ }^{338}$

\footnotetext{
${ }^{337}$ Eine ähnlich ungewöhnliche Affektanalogie bietet auch Marinas Kollege John in Bezug auf seine erste Gorillabegegnung: ,[I]ch starrte nur in diese großen braunen Augen da oben und dachte: Wow. Es ist wie eins dieser gigantischen Bilder, Oklahoma City Bombing, oder wenn eine Atombombe explodiert, und du denkst nur: Wow“ ( $A W$, S. 87). Diese Anspielung auf das (hier: schreckliche) Erhabene wird aber nicht weiter aufgegriffen.

${ }^{338}$ Auf den Anblick von Griffin Wilder im Freilandoutfit via Skype reagiert sie bspw. mit einem „,warmen[n] Kribbeln [...] zwischen meinem Bauch und meiner Schamgegend“ und konstatiert: „Kein Wunder, dass er mich so lange um den Finger wickeln konnte“ (AW, S. 231).
} 
Penibel listet Marina die Bedingungen der wissenschaftlichen Forschung am Institut auf:

Wenn man jeden Abend und jedes Wochenende arbeitet [...] wird Schlaf zu einer trostreichen Angelegenheit für die Leute, die es nicht schaffen, ihre Träume zu leben. [...] Wenn man sich dem hohen Qualitätsanspruch der Spitzenforschung stellt, wird Freizeit zu etwas, das andere Leute auf Messen besichtigen [...]. Wenn man rund um die Uhr arbeitet, befriedigt man auch sein Zugehörigkeitsbedürfnis besser auf der Arbeit, mit Gleichgesinnten, ehe man depressiv wird, kriminell oder suizidal. (32)

Die Formulierung dieser Bedingungssätze klingt bei Marina zunächst wie Kritik an den Arbeitsbedingungen der primatologischen Forschung am Institut - kippt dann aber um in die Affirmation. Marina zieht Stolz daraus, auf diese Art und Weise - ohne Freizeit, ohne Sozialleben jenseits der beruflichen Kontakte, auch ohne Schlaf - zu arbeiten, sich der Aufgabe hinzugeben. Diese ambivalente Strategie von Kritik und Affirmation zieht sich durch den Roman. Bereits zu Beginn heißt es:

Glaub bloß nicht, es sei leicht, sich seine [Griffin Wilders] Gunst zu erarbeiten. Du hast mit Auszeichnung studiert und Empfehlungen bekommen? Das hat die halbe Welt. Du machst Überstunden und dich nützlich, nimmst dabei klaglos hin, dass du nie auftauchst unter den Ko-Autoren und in den Danksagungen, auch wenn $d u$ die Daten erhoben hast, nicht Griffin? Erwarte kein Schulterklopfen für diese Selbstverständlichkeit. Es ist ein Akt der Gnade und nicht weniger, wenn dein Name eines Tages neben seiner Tür zu lesen ist, The super student is: Marina Heuter. Dann koste deine fünfzehn Minuten mit aller Macht aus. Sind sie erst vorbei, führt kein Weg zurück. (11, Hervorheb. im Original)

Die Gunst des primatologischen Gottes ist eine Gnade, die Aufopferung für sein Projekt und die wissenschaftliche (Selbst-)Ausbeutung eine Selbstverständlichkeit, Anerkennung ist kurzer, flüchtiger Ruhm. Ein solcherart funktionierendes System begünstigt in der Protagonistin vor allem eine affektive Regung: Neid.

Dieser Neid richtet sich - in weiterer Affirmation der Verhaltensbiologismen, denen Marina anhängt - auf andere Frauen im Wissenschaftsbetrieb und diejenigen Figuren wie den Doktoranden Arne Müller, die in Marinas Augen ihr Verhältnis zu dem zugleich bewunderten und gefürchteten Alphamännchen Griffin gefährden. Bei ihrem ersten Einsatz als Dolmetscherin soll sie in einem Versuch zwischen der Diplomandin Sandra und dem Gorilla Yeh-teh übersetzen. Sandra wird sogleich unter der Kategorie der Konkurrenz begutachtet: „[Sie] war zwei Jahre älter als ich und konnte nur hoffen, in ein oder zwei Jahren da zu sein, wo ich jetzt schon war" (63). Marina liest die Kommunikation zwischen ihr und Sandra ausschließlich als taktierendes Abwägen der Qualifikationen der anderen, und fürchtet sogleich um ihren Status in der Institutshierarchie. Daraus resultiert die Abwertung der Anderen: „Ich hätte sie gern gefragt, in welchem Ghetto sie denn aufgewachsen war, wie ihre Erklärung dafür lautete, dass sie mit fünfundzwanzig ihr Studium noch nicht beendet hatte" (63). Sie scheut jedoch die direkte Auseinandersetzung und reagiert stattdessen innerlich trotzig, als Yehteh gebärdet: „Sandra ist sehr schlau“ (64). Hierauf heißt es: „Ich war genauso 
schlau wie Sandra“, und auch als Yeh-teh Sandra ein zweites Mal „bewundernd“ (66) anblickt, weil sie ihm zeigt, was er im Schreibversuch falsch gemacht hat, vermerkt Marina wieder: „Ich konnte es auch, aber das würde ich schön für mich behalten“ (66). Marina neidet Sandra Yeh-tehs Bewunderung und sorgt daher subtil dafür, dass Yeh-tehs Frustration über die Versuchssituation größer ausfällt, als sie müsste.

Bei der Programmkoordinatorin bei UNEP in Genf, Teresa, steht demgegenüber die äußere Erscheinung und das Arbeitsethos im Vordergrund. Sie wird von Marina wie folgt beschrieben:

Wenn sie glaubt, etwas Kluges gesagt zu haben, worauf niemandem eine Antwort einfallen wird, reckt sie ihr spitzes Kinn in die Höhe, sodass die konkav um ihre untere Gesichtshälfte geföhnten Haarsträhnen nach hinten fliegen. Ein herausforderndes Funkeln aus dunkel geschminkten Augen, ein selbstgefälliges Lächeln auf roten Lippen. (20)

Damit unterscheidet sich die ,Externe‘ von den Frauen der Primatologie:

Am Institut trifft man eher Frauen wie mich, ungeschminkt, mit bunten Bändern in den Haaren, Frauen ohne Haarschnitt, deren Kleidung man die Outdoor-Erfahrung ansieht. Woher nimmt sie den Glauben, dass ausgerechnet sie sich Make-up leisten kann, ohne intellektuell unterschätzt zu werden? Oder weiß sie, dass das kaum möglich ist, und versucht es gleich mit anderen Mitteln? (20)

Dieser Überlegung geht die Information voran, dass Marina Teresa „hasst“ (20), weil diese gern auf Inseln in den Urlaub fährt, und: „Beim Wort ,Urlaub“ schießt mein Blutdruck einfach in die Höhe. Kann man nicht machen“ (20). Zudem ist Teresa mit Marinas Kollegen John befreundet, ,[b]ei ihren Kontrollbesuchen übernachtete sie sogar in seiner Wohnung"(19). Ebenso wie das selbstbewusste Auftreten sind Urlaub und die Beziehung zu John hier Glücksgüter, die Marina Teresa neidet - genug Anlass für sie, die andere Frau in ihrer Darstellung herabzusetzen.

Den Doktoranden Arne Müller findet Marina ,viel jünger, als ich ihn mir vorgestellt habe, noch jünger, als ich es war, als Griffin mich unter seine Fittiche nahm. Ein Wunderkind. Seine Eltern müssen wahnsinnig stolz auf ihn sein“ (67). Sie fühlt sich sogleich von ihm ,belehrt“ (67) und neidet ihm, dem Mitarbeiter Griffins am Institut, die „,selbstsichere[] Natürlichkeit, als wäre er hier [im Leipziger Institut] zu Hause“ (67 f.). Als Arne Müller sie duzt, heißt es: „Ich kann es nicht fassen und werfe ihm einen strafenden Blick zu: Du dutzt mich nicht!“ (68). Als Arne Müller sich daraufhin korrigiert, nimmt sie auch daran Anstoß, denn es geschieht ,in einem Ton, als würde er mir einen Gefallen tun“ (68). Endgültig verdorben hat es sich der Doktorand mit Marina, als er Griffin Wilder beim Vornamen nennt, denn: „Drei Jahre hat es gedauert, bis ich ihn so nennen durfte“ (70).

Diese auffällige hierarchische Prägung des emotionalen Verhaltens deutet darauf hin, dass es hier nicht schlicht um die Rivalität im Rahmen jenes beruflichen Wettbewerbs geht, den der Wissenschaftsbetrieb durch seine Flaschenhalsstruktur ausgerufen hat. Bereits in Aristoteles' Rhetorik ist die Voraussetzung für Neid die 
relative soziale Gleichheit von Neidenden und Beneideten. ${ }^{339}$ Neid ist die Unlustempfindung angesichts der Glücksgüter anderer, die man selbst zu verdienen glaubt, ${ }^{340}$ ähnlich wie dies auch in der - von Aristoteles und in der Folge vergleichsweise aufgewerteten - Rivalität der Fall ist. ${ }^{341}$ Der Unterschied zwischen Neid und Rivalität in Aristoteles' ,Logik des Neides ${ }^{642}$, liegt im Anliegen des Empfindenden: ,[D]enn der eine strebt aufgrund der Rivalitätsempfindung danach, die Güter zu erlangen, der andere aber aufgrund seiner Neidempfindung, daß sein Mitmensch sie nicht besitze“. ${ }^{343}$ Knut Eming sieht bei Aristoteles die Gemeinsamkeit zwischen Neid und Rivalität, „das jeweilige Gut leidenschaftlich zu bejahen, aber den wesentlichen Unterschied, dass der Neider anders als der Rivale um seine ihm fehlenden Möglichkeiten weiß“ “. ${ }^{344}$ Entscheidend ist, dass

\begin{abstract}
der Neider [...] [nicht] den Besitz [begehrt] [...], sondern er begehrt, dass der andere das Glücksgut nicht hat. Da er das aber nicht zu bewirken vermag, nagt an ihm das Gefühl der Zurücksetzung. Der Neider erleidet somit eine Kränkung (lype), denn ihm wird eine Anerkennung vorenthalten, worüber er sich aber nicht beschweren kann, weil er dann wie der Rivale seinen Gegner (den Beneideten) offen und direkt angreifen müsste. Daher greift er zur Herabsetzung und spricht dem Beneideten ab, verdientermaßen das Glücksgut erworben zu haben. Letztlich will er eigentlich, dass das beneidete Gut zerstört wird, um so seine eigene Zurücksetzung aufzuheben und den Rangunterschied zwischen sich und dem Beneideten zu nivellieren. ${ }^{345}$
\end{abstract}

In den obigen Textbeispielen nimmt Marina eine zumindest partielle, ihren Selbstwert bedrohende Gleichheit zwischen sich und den Kritisierten (Arne Müller als Nachfolger Marinas in der Gunst Griffins; Sandra als Mitarbeiterin im Projekt und konkurrierende Interaktionspartnerin der Gorillas; Teresa als andere Frau) an. Sie ist vor dem Hintergrund einer solchen angenommenen Gleichheit aber damit beschäftigt, einen Rangunterschied auszumachen und wahlweise zur Abgrenzung herzustellen oder zu ihren Gunsten zu nivellieren. Marinas Verhalten in Auswilderung lässt sich mit Emings Definition des Neiders als neidische Missgunst

${ }^{339}$ „Neid empfinden werden solche, denen gewisse andere gleich sind oder doch erscheinen“; „Gleich-sein definiere ich [als Gleichheit] hinsichtlich Herkunft, Verwandtschaft, Lebensalter, Verhalten, Ruf, Besitz“ (II.10, 1387b; Hervorheb. im Original); „Neid empfindet man nämlich denen gegenüber, denen man der Zeit, dem Ort, dem Lebensalter und dem Ruf nach nahe steht" (II.10.1388a). Aristoteles: Rhetorik. München 1989, S. 116 f.

${ }^{340}$ Vgl. Harreß, Birgit: Einleitung. In: Dies. (Hg.): Neid. Darstellung und Deutung in den Wissenschaften und Künsten. Berlin 2010, S. 7-15, hier S. 7.

${ }^{341}$ „Rivalität [ist] etwas Edles und edlen Menschen eigentümlich, das Beneiden aber etwas Niedriges und niedrigen Menschen eigen“(II.1.1388a). Aristoteles: Rhetorik, S. 118.

${ }^{342}$ Vgl. Eming, Knut: Die Logik des Neids. Aristoteles über das an uns nagende Gefühl der Ungleichheit. In: Birgit Harreß (Hg.): Neid. Darstellung und Deutung in den Wissenschaften und Künsten. Berlin 2010, S. 17-31, hier S. 21.

${ }^{343}$ Aristoteles: Rhetorik, S. 118 f. (II.11.1388a-b).

${ }^{344}$ Eming: Die Logik des Neides, S. 24.

${ }^{345}$ Eming: Die Logik des Neides, S. 26. 
verstehen: Sie begehrt weniger, die Glücksgüter der anderen (Yeh-tehs und Griffins Anerkennung, ein attraktives Äußeres und Johns Freundschaft) zu erlangen, als dass diese sie gar nicht erst hätten oder nicht mehr besitzen sollen. Dass Eming in seiner Beschäftigung mit Aristoteles' Neidlogik als illustrierendes Beispiel für eine Neidsituation ausgerechnet eines aus dem akademischen Betrieb heranzieht, verweist darauf, weshalb die Interpretation von Marina als aristotelischer Neiderin leicht fällt: ${ }^{346}$ Marina ist geprägt von dem sozialen Umfeld, indem sie wissenschaftlich erwachsen wird, d. h. dem akademischen System und dessen Affektkultur.

Bettina Suleiman illustriert mit Marina in Auswilderung, dass Neid ,den Versuch einer Selbstbehauptung, freilich einer unglücklichen, gescheiterten Selbstbehauptung“"347 zeigt, wie Birgit Harreß schreibt. Das Selbstbehauptungsbedürfnis scheint aus dem System des wissenschaftlichen Betriebes zu erwachsen. Dieses bezeichnet Marina selbst als „eine Art Zweiklassensystem“ (88), welches nicht nur zwischen denjenigen,, „die sich mit Tieren auskannten, Biologen, Zoologen, Primatologen, Verhaltensforscher[n] usw.“ (88), und den anderen, „de[m] Support Staff, Verwaltungsmitarbeiter[n], Statistiker[n], Techniker[n] und eben Dolmetscher[n]“ (89), unterscheidet, sondern auch zwischen den erfolgreichen Wissenschaftler/innen und den „Versager[n]“ (111). Erstere sind Primatolog/innen wie Griffin Wilder, die ein Institut und etliche experimentelle Forschungsprojekte im Labor leiten. Letztere sind in Marinas Verständnis deutlich: die Feldforscher/ innen. Ein Feldposten habe früher als ,letzter, aber sicherer Ausweg für alle Versager, die sonst nirgendwo zu gebrauchen waren“"(111), gegolten, jetzt sei er nur mehr ein „schlecht bezahlter Verwaltungsposten im Busch“(111). Erst später stellt sie fest, dass John, der nach Uganda gegangen war, weil am Ende des ersten Forschungsprojektes nicht die seiner Ansicht nach indizierte Empfehlung für die „Anerkennung der Subjects als Personen“ (111) stand, dort wohl glücklicher gewesen ist als sie in ihrer Arbeit am DIAG. So wirft sie ihm schließlich, wiederum retrospektiv neidisch, vor, „[d]ass ich sieben Jahre lang an meinem Schreibtisch verrottet sei, während er friedlich mit allen Tieren im Paradies gelebt habe“ (249). ${ }^{348}$ Marinas Selbstbehauptungsbestreben dient so nicht dazu, ihr die vermeintlichen Glücksgüter einzutragen, sondern äußert sich in einer neidischen strategischen Herabsetzung der anderen, die letztlich nicht dazu beiträgt, dass Marina sich besser fühlt oder mehr erreicht, sondern sie in der Unzufriedenheit verharren lässt.

\footnotetext{
${ }^{346}$ Vgl. Eming: Die Logik des Neides, S. 27.

${ }^{347}$ Harreß: Einleitung, S. 7.

${ }^{348}$ Diese Einschätzung entsteht auf der Insel, auf der die Gorillas ausgewildert werden sollen. Bei ihrem Wiedersehen bemerkt Marina über John: „Seine Bizepse sehen nach echter körperlicher Arbeit aus und nicht nach Fitnessstudio, nach einem Mann, der einen wirklich beschützen könnte auf dieser einsamen Insel“ ( $A W$, S. 170). Er wirkt ,jugendlicher und um einiges fitter“, „braungebrannt und mit schärferen, heldenhaften Gesichtszügen [...]. Seine Arbeit erledigt er vermutlich im Freien, in einer Baumkrone hockend und zahme Affen, Elefanten und Kobras versammeln sich um ihn und schauen ihm über die Schulter dabei“ (S. 170). Diese deutliche Anspielung auf die Tarzan-Figur entspricht wohl einem Selbstbild von Marina, die sich jedoch nicht entscheiden kann, ob sie Tarzan eine Jane oder King Kong (dem Gorilla Yeh-teh) eine Anne sein will.
} 


\subsubsection{Affektive Manipulationen}

Nicht nur steuert derart eine von institutionellen Strukturen begünstigte, wenn nicht sogar produzierte Affektkultur Marinas individuelles Empfinden und ihr professionelles Verhalten. Auch ihre Beziehung zu den ,Subjects“, deren Gesten sie interpretieren und mit denen sie im Sinne der Projektziele kommunizieren soll, wird durch Gefühle und Interessen bestimmt. Entgegen ihrer Überzeugung des intuitiven, empathisch fundierten Wissens sind Marinas Interpretationen der Gorillagesten Auslegungen im Sinne des gewünschten Forschungsergebnisses oder ihrer eigenen Bedürfnisse. Zunächst betrifft dies Belege dafür, dass Gorillas die Kriterien des Personenstatus nicht erfüllen, später deren Eignung für die Auswilderung. Dies wird schon recht früh im Text deutlich. Als Marina zu Beginn ihres Berichts auf einem Video sieht, wie die Gorillas des ersten Auswilderungsversuches aus dem Gehege ausbrechen und sich auf einen Marsch machen, heißt es: „Von Zivilisation ist weit und breit keine Spur und mir kommen fast die Tränen vor Rührung. [...] [I]ch kann ihre Gesichter nicht erkennen, aber ich bin mir sicher, dass sie ganz aus dem Häuschen sind vor Glück“ (11). Marina begeistert sich für die Zivilisationslosigkeit, unterstellt deswegen den Gorillas Glück angesichts ihrer Situation - in ihrem vertrauten Leipziger Gehege einzuschlafen und mitten in einer Savanne aufzuwachen - und verliert darüber aus dem Blick, was es bedeutet, dass die Gorillagruppe, die sie auf den Videoaufzeichnungen sieht, gerade ,[m]it der Disziplin und Hingabe von Pilgern“ (12) Richtung Zivilisation flüchtet. Glücklich sind diese Gorillas wohl erst, als sie „Anzeichen menschlicher Aktivität“ (13) mit „Jubelschreie[n]“ (13) quittieren.

Entgegen Griffin Wilders „Credo“, „[m]an müsse sich während der Forschung zwingen, die menschlichen Beziehungen [zu den Tieren] abzustellen [...]. Das könne man später machen, wenn man ,die Seite wechselt", wie er das nannte, wenn man zum Tierschützer wurde“ (41), unterhält Marina jenes affektive Verhältnis zu den Gorillas, welches hier ,menschlich` genannt wird. Besonders das Männchen Yeh-teh, mit dem sie in der ersten Versuchsreihe zusammengearbeitet hat, ist ihr freundschaftlich vertraut. Sie rechtfertigt dies auch forschungspraktisch aus den Prozessen ihrer Arbeit heraus:

Aber wenn man sieben Jahre lang mit jemandem arbeitet, wird der Umgang unweigerlich vertraut. Ich verstand nicht nur, was die Subjects sagten, ich wusste auch, was sie meinten. $[\ldots][\mathrm{M}]$ it mir redeten sie überhaupt nur, weil sie mich mochten, und nicht etwa, um der Wissenschaft zu dienen. Das waren doch keine Roboter. Der Übergang zwischen Experiment und freundschaftlicher Unterhaltung war unvermeidbar fließend. (41)

Die Notwendigkeit zu dieser Form der Beziehung ist im Wesen von Martinas Arbeit am Institut begründet: Kommunikation stellt eine Beziehung zwischen den Kommunizierenden her; je komplexer das Kommunizierte, desto notwendiger wird diese Beziehung für dessen Verständnis:

Mit Schaubildern, gezeigt von unbekannt, konnte man einzelne Vokabeln kommunizieren, komplexere Gespräche jedoch bedurften einer menschlichen Beziehung. Wir mussten mit Blicken und Gesten Kontakt aufnehmen, mit unseren Körpern [...]. Ob es Griffin passte oder nicht, anders konnten seine Experimente nicht durchgeführt werden. (41) 
Im Verlauf des Auswilderungsprojektes sieht auch Griffin ein, dass es einer Beziehung zwischen Forschenden und Beforschten bedarf, um die Gorillas davon zu überzeugen, in die Wildnis zu gehen. Selbst wenn durch die praktischen Bedingungen der Gebärdensprache die ,theoretische Möglichkeit, dass ich den Subjects hier und da unbeabsichtigt mittels meiner Körpersprache einen kleinen Tipp hätte geben können“ (41), besteht, werden Marinas „Gespräch[e] mit einem Vertreter einer fremden Spezies“ (63) somit zum rhetorischen Instrument für die Umsetzung des Forschungsziels. Im Rahmen der Fiktion wird in Auswilderung also nicht der tier-menschliche Rapport zum Problem, sondern Marinas interessensgeleitete Auslegung und Kommunikation dieses Rapports.

Wie bereits im obigen Zitat anklingt, nimmt Marina nicht nur an, dass sie verstehe, „was die Subjects sagten, ich wusste auch, was sie meinten“ (41, Hervorheb. MS). Ähnlich wie in Bezug auf ihre selbstbekundete Empathiefähigkeit geht sie hier, zusätzlich gestützt durch das Beziehungsband, davon aus, alleinig zu verstehen, was die Tiere wollen - und was für diese , das Beste' sei. Sie behauptet dies sogar im Angesicht besserer Argumente, so wenn sie, wie bereits erwähnt, jenen Hinweis des Verhaltensbiologen John auf die „neophoben Tiere“ (89) als ,Mansplaining ‘ aus dem Weg räumt. Wo eine affektiv gestützte Hermeneutik in der Feldforschung zur ,Lesbarkeit" von Primatenverhalten führen mag (vgl. Abschn. 3.2.3), ist sie vor dem Hintergrund der experimentellen Forschungssituation, um die es im Roman geht, ethisch problematisch, wie Marina selbst feststellt: „Man sollte nie unterschätzen, dass die Korrektheit oder Fehlerhaftigkeit einer Übersetzung manchmal alles entscheidet, ein ganzes Leben“ (48). Es steht in Auswilderung dabei nichts Geringeres auf dem Spiel als das (Über-)Leben der Gorillas.

Was auf den ersten Blick wie die Rache einer enttäuschten Akademikerin an ihrem Arbeitgeber wirken mag - Marinas Anzeige unethischer Forschungspraktiken und ihrer vermeintlich im Dienste Griffins vorgenommen Manipulationen -, stellt sich dann im Fortschreiten ihrer Erzählung als Grundzug von Marinas Forschungsethos und als Zuspitzung ihrer unzuverlässigen Erzählerinnenperspektive heraus. Sie enthüllt selbst, wie stark sie manipulativ in die Versuche mit den Gorillas eingegriffen hat, um das gewünschte Ergebnis herbeizuführen. Bereits bei ihrem ersten Einsatz in einem Versuch heißt es: „Gerade weil Yeh-tehs Scheitern das erwartete Ergebnis war, musste der Versuch so korrekt wie möglich durchgeführt werden [...]. Wir mussten uns wirklich bemühen, dem Subject etwas beizubringen“ (66). Marina aber sorgt durch emphatische Übersetzungen (,Nein, das stimmt ganz und gar nicht, mit offenem Mund, übertrieben, geschauspielert", 66) für eine erhöhte Frustration des Versuchstieres und dadurch für eine höhere Wahrscheinlichkeit des Scheiterns. Bei der Sichtung alter Versuchsprotokolle im 7. Kapitel wird deutlich, dass Marina den äffischen ,Subjects' zudem die ,richtigen“ Antworten auf die ihnen gestellten Fragen suggeriert hat:

Vergesslichkeit, hatte ich ihm [Yeh-teh] kurz davor verraten, bedeute, die Prüfung zu bestehen. (164) 
Wir machten jeden Versuch zweiundsiebzig Mal, einmal mit jedem Subject, und nach ein paar Malen konnte ich mir zusammenreimen, was Yeh-teh sagen müsste, um auf der sicheren Seite zu sein; der Seite, die in den Dschungel führte und nicht in den Zoo. Dann musste ich nur noch rechtzeitig vor Versuchsbeginn einen günstigen Moment abpassen, um ihm die richtigen Antworten zu verraten. (165)

Weil Marina von Griffins Projekt nicht nur überzeugt, sondern begeistert ist, weil sie sich, vor Freude fast in die Hose macht', weil sie an die impliziten Ziele der Forschung religiös glaubt, beeinflusst sie die Versuche durchweg im Sinne des gewünschten negativen Ergebnisses für die Anerkennung der Personalität und arbeitet auch im Rahmen des Auswilderungsprojektes mit Fehlübersetzungen, um sowohl Griffin Wilder und Arne Müller als auch den Gorilla Yeh-teh in ihrem Sinn zu manipulieren. Dass dahinter letztlich Marinas eigener Wunsch nach Ausbzw. Verwilderung steckt, realisiert sie selbst erst spät. Die unbewusst ersehnte Befreiung von Hierarchie und Erfolgsdruck der akademischen Welt, von deren Affektkultur und den gesellschaftlichen Einschnürungen, die sie empfindet, gesellt sich dem Gefühl für die andere Kreatur bei, das Marina als Begründung sieht, im Sinne des ,Besten“ für den Gorilla zu handeln (,Ich will doch nur dein Bestes“, 165). Auch wenn sie dann, wie sie später selbst sieht, nicht zu Yeh-tehs Besten handelt, sondern im Sinne ihrer Wunschvorstellung. Diese Einsicht beendet einen langsamen Prozess der individuellen Desintegration, den man in ihrer Erzählung untergründig verfolgen kann. Sie reicht von ersten Sprachschwierigkeiten $(19,26)$ über anhaltende Schlaflosigkeit $(129,130 \mathrm{f} ., 161)$ und die körperliche Symptomatik eines erschöpften Systems (156 f., 161), bis hin zur Verwahrlosung, Kommunikationsunfähigkeit (199 f.) und Infantilisierung. ${ }^{349}$ Der Endpunkt einer solchen Desintegration ist das Ankommen auf der Insel. Auch hier sorgt Martina noch durch ihre Manipulationen der Ethikkommission dafür, dass sie auf der Insel verbleiben darf und handelt damit in ihrem, nicht in Yeh-tehs Sinne. Denn dieser ist nur ihretwegen auf der Insel: Er ist durch seinen ,Beschützerinstinkt ${ }^{*}$, der letztlich auf einer emotionalen Manipulation des Affenmannes durch die Menschenfrau beruht, an sie gebunden. Gegen die in dieser Komposition implizite ,Interspecies-Romance' immunisiert schließlich die vollzogene Regredierung zur Kindlichkeit. Auf der Insel braucht Marina kaum Kleidung, muss sich - wie in den schönsten Erinnerungen an ihre Kindheit ${ }^{350}$ - nicht mehr waschen, spricht passenderweise von ihrem Treuhänder als ihrem „Vormund“ (248) und lässt sich von dem zum Festland aufbrechenden John am Handgelenk halten, „wie man ein

\footnotetext{
${ }^{349}$ Neben dem Rückzug in Angewohnheiten aus ihrer Kindheit zeigt sich die Infantilisierung vor allem in Marinas hilflosen Reaktionen auf das Verhalten anderer: Mehrfach wird Griffins Ton z. B. als ,gemein“ bezeichnet, bspw.: „Sein Ton ist so gemein, dass ich am liebsten auf der Stelle in Tränen ausbrechen würde“ (AW, S. 152), „Wie gemein“ (S. 192).

${ }^{350}$ Diese Erinnerungen drehen sich um die Freiheit von (hygienischen) Regeln, etwa sich nicht waschen und sich nicht die Zähne putzen zu müssen, oder ins Wasser zu urinieren, anstatt eine Toilette aufzusuchen (vgl. AW, S. 245).
} 
kleines Kind festhalten würde, um es vor Dummheiten zu bewahren“ (259). Der Gorilla Yeh-teh erscheint ihr konsequenterweise zum Schluss ,weich und kuschelig wie ein riesiges Stofftier“"(264).

\subsubsection{Was will die Gegenwartsliteratur mit der Primatologie?}

Ulrike Draesner elaboriert in Sieben Sprünge vom Rand der Welt, wie die Vergangenheit das Fühlen bestimmt. Anhand der Familien Grolmann und Nienalt illustriert sie die Reichweite transgenerationaler Traumata: Erstens zeigt sie die Übertragung spezifischer emotionaler Phänomene von Eltern auf Kinder (und Enkel/innen). Zweitens äußern sich diese genealogisch-historischen Konditionierungen in verschiedenen Verhaltensmustern und Haltungen gegenüber Emotionalität, die in Sieben Sprünge ein Nachdenken über den Sinn und Zweck von Gefühlen, besonders von Empathie als ,conditio humana' anregen. Drittens, und darin geht Draesners Roman über eine literarische Ausführung der in der psychologischen und psychotherapeutischen Forschung zutage geförderten Erkenntnisse über transgenerationale Traumata hinaus, stellt der Text die Frage danach, welche Rolle die Affektregime von Kriegskindern und die Traumata, die sie weitervererbt haben, im beruflichen, in diesem Fall im wissenschaftlichen Bereich spielen. Draesner verhandelt dabei wie bereits in anderen ihrer Texte erneut den Zusammenhang von (Natur-)Wissenschaft, Gesellschaft und Individuum: ${ }^{351}$ In welchem Zusammenhang stehen das anthropologische Forschungsinteresse der Primatologie und soziokulturell und historisch bedingte emotionale Persönlichkeitsprofile? Das ist auch eine Frage danach, wie ein Ethos als Haltung der (eigenen) Emotionalität gegenüber die Erkenntnisgewinnung der (primatologischen) Forschung bestimmt. Die Hinwendung beider primatologischer Figuren zur Affektivität - bei Eustachius als um das späte Nachholen lang entbehrter Nahaffekte bemühtes ,humanistisches“ Projekt, bei Simone als epistemologische Auseinandersetzung mit der Rolle von Emotionalität bzw. Empathie in der Erkenntnisgewinnung von Menschen an Affen - ist auch eine durch das Familiärbiografische bedingte ethische Wende in ihrer Forscher/innen-Biographie.

Bettina Suleimans Auswilderung verhandelt auf ganze andere Art ebenfalls, wie Ethiken der Emotionalität die Möglichkeiten der Erkenntnisgewinnung in der primatologischen Forschung bedingen. Die Rolle, welche die Erzählerin Marina als Mitarbeiterin eines Forschungsprojektes für diese Erkenntnisgewinnung spielt, ist in Auswilderung deutlich davon abhängig, wie sie fühlt und wie diese Affektivität ihre Handlungen steuert. Anhand von erstens Marinas (populär)biologistisch determiniertem Selbstbild, zweitens ihrem Verhältnis zu sich selbst und Anderen in der institutionell geprägten Gefühlskultur der Wissenschaft und drittens der

\footnotetext{
${ }^{351}$ Vgl. Catani, Stephanie/Marx, Friedhelm: Vorwort. In: Dies. (Hg.): Familien, Geschlechter, Macht. Beziehungen im Werk Ulrike Draesners. Göttingen 2008, S. 7-11, hier S. 7 f.
} 
durch das Affektive geleiteten manipulativen Handlungsweisen in der Forschungspraxis problematisiert Suleiman die Rolle von Emotionalität aus einer forschungsethischen Perspektive heraus. Dies zeigt sich auch daran, dass der Roman anhand anderer Figuren thesenhaft zwei alternative Positionen formuliert, wie ein Ethos der Emotionalität in der primatologischen Forschung aussehen kann: Zum einen äußert John auf Basis seines ethologischen Wissens Bedenken hinsichtlich der Chancen der Auswilderungspolitik des Instituts und Großprojektes: „Eine Art Rückkehr in die Wildnis ist kein realistisches Szenario [...] Das sind neophobe Tiere. Eine Umsiedlung in die Wildnis würde für sie Leid bedeuten“ (89). John sieht auch die moralischen Konsequenzen des ersten Forschungsprojektes zum Personenstand:

\begin{abstract}
Wenn Menschenaffen als Personen anerkannt werden, kannst du nicht mehr gegen ihre Interessen handeln. Das ändert sich. Sie werden rechtliche Betreuer haben, die verpflichtet sein werden, in ihrem besten Interesse zu handeln. [...] Du kannst nicht mehr auf ihre Kosten Forschungspreise gewinnen, in Nature und Animal Behavior veröffentlichen, dein Ego befriedigen. (90, Hervorheb. im Original)
\end{abstract}

Indem er diese Konsequenzen aufzeigt, stellt John zum einen ein ethisches Verhältnis zum Tier vor, das auf einer Logik der Empathie als Perspektivübernahme basierende moralische Argumente ins Feld führt: Die Tiere können Leid empfinden. Die Tiere sind neophob. Folglich würde eine Umsiedlung in eine ihnen unbekannte Umwelt diese neophoben Tiere leiden lassen. Die auf wissenschaftlichen Erkenntnissen beruhende Verantwortung für das Wohlergehen der Gorillas steht vor ihrem Gebrauch in der Wissenschaft. Der Roman greift darin auf den Konnex von Empathie und Ethik zurück, wie er für die Empathieforschung derzeit ausschlaggebend ist. Coplan und Goldie sehen Empathie etwa nicht nur als Befähigung zur, Theory of Mind" und zur Vorhersage und Erklärung des Denkens, Fühlens und Verhaltens anderer, wie John sie hier anwendet. Sie bezeichnen Empathie auch als ,important in relation to our capacity to respond to others ethically“. ${ }^{352}$ Zum anderen stellt John im Sinne dieses Forschungsethos die Motivation der im Projekt Forschenden infrage: Ein positiver Befund zur Frage der Personalität würde de facto und de jure auf eine Selbstabschaffung der bestehenden experimentellen Forschung mit Menschenaffen hinauslaufen. Eine Unvoreingenommenheit der in diesem Projekt Urteilenden kann daher gar nicht gewährleistet sein. Es ist dann wiederum nur konsequent, dass sich John zunächst aus der experimentellen Forschung zurückzieht und nach Uganda ins Feld geht, wo sich Fragen nach der Forschungsethik zumindest anders stellen als am Leipziger Institut. Johns Position in Auswilderung appliziert die von Lori Gruen geforderte verflochtene Empathie (,entangled empathy') im Rahmen einer neuen Tierethik als:

\footnotetext{
${ }^{352}$ Coplan/Goldie: Introduction, S. ix.
} 
ein[] komplexer Erkenntnisprozess, bei dem Individuen, die mit dem Wohlergehen anderer empathisieren, zunächst auf den Zustand des anderen Individuums eingehen [...] und sich dann unter Einbeziehung der Reflexion in die Lage des anderen hineinversetzen. Sie beurteilen dann, inwiefern die Umstände, in denen sich das andere Individuum befindet, $\mathrm{zu}$ dessen geistigem Zustand beitragen oder dessen Interessen beeinflussen können. Um diese Urteile zu treffen, muss die empathische Person die offensichtlichen Merkmale der Situation auswerten. Dazu ist erforderlich, dass sie versucht herauszufinden, welche Informationen relevant sind, um effektiv mit dem betreffenden Wesen zu empathisieren. Sie muss auch die Möglichkeit im Blick haben, dass sie nur ihre eigenen Interessen oder Wünsche auf die des anderen projiziert. Verflochtene Empathie involviert also sowohl Affekt als auch Kognition und üblicherweise motiviert sie zum Handeln. ${ }^{353}$

Steht John in Auswilderung für eine empathische, vernünftige Position zur Affenforschung und ihren tierlichen Akteur/innen, verkörpert Griffin zumindest partiell einen stoisch anmutenden Gleichmut im Umgang mit Erfolg oder Nicht-Erfolg:

Vergiss einfach nie, dass das, was du willst, und das, was ist, zwei verschiedene Dinge sind. Übe dich in Gleichgültigkeit. Lass sein, was ist, und dann notiere es, sortiere es, publiziere es. Es ist doch spannend herauszufinden, wie die Dinge sich entwickeln, wenn man nicht in sie eingreift. Auch wenn du mal falsch liegst. Dann lernst du eben dazu. [...] Du musst die Dinge einfach interesselos betrachten und nehmen, was kommt. (238)

Diese Passage scheint antike Epistemologie und Affektlehre zu kombinieren: Das Streben nach Erkenntnis begegnet der stoischen Apatheia als tugendhafter Haltung zur Regulierung des anthropologischen Wissensverlangen. ${ }^{354}$ Griffins wissenschaftliches Credo zeigt jedoch vor allem das herrschende affektive Leit- und Selbstbild primatologischer Forschung in Auswilderung auf. Es fordert möglichst umfassende Objektivität in der Forschungspraxis durch die Unterdrückung (und nicht wie bei Aristoteles: Mäßigung) affektiver Anteile. Dabei nimmt die Figur Griffin jenes Dogma der Objektivität als epistemischer Tugend auf, wie sie Lorraine Daston und Peter Galison als seit Mitte des 19. Jahrhunderts sich etablierendes Ideal in den Naturwissenschaften identifizieren und wie es in der Einleitung bereits diskutiert wurde (vgl. Abschn. 1.2): Erst die Entdeckung von Subjektivität als mögliches Problem für die Erschließung und Abbildung der Natur macht Objektivität als ihr vermeintliches Gegenteil in den Augen der modernen Naturforschung notwendig. Das Ziel ist die Selbstdisziplinierung der Forschenden, um den ,human factor', den Eustachius Grolmann in Sieben Sprünge wieder radikal ins Experiment integrieren will, zuallererst herauszurechnen: „First and foremost, objectivity is the suppression of some aspect of the self, the countering of subjectivity. [...] [T]he emergence of objectivity must tally with the emergence of a certain kind of willful self, one perceived as endangering scientific knowledge. ${ }^{\text {(355 }}$

\footnotetext{
${ }^{353}$ Gruen: Sich Tieren zuwenden, S. 398 f.

${ }^{354}$ Zum Wissensverlangen vgl. Lachmann, Michael: Problematik: Nichtwissen und Wissensverlangen im philosophischen Bewußtsein. Göttingen 1949.

${ }^{355}$ Daston/Galison: Objectivity, S. 36 f.
} 
Wie Griffins Position der interesselosen Erkenntnis verdeutlicht, führt der Weg zu einer solchen modernen Objektivität aber über seit der Antike diskutierte Praktiken des emotionalen Managements im Rahmen ethischer Überlegungen. Auch Daston und Galison sehen eine solche Verwicklung von epistemischen und ethisch Ansprüchen und Techniken des Selbst als Ergebnis der von ihnen identifizierten epistemologischen Wende im 19. Jahrhundert: ,[I]f objectivity and other epistemic virtues were intertwined with the historically conditioned person of the inquirer, shaped by scientific practices that blurred into techniques of the self, moralized epistemology was just what one would expect. “356

Griffin Wilder lässt sich damit als Vertreter eines älteren Paradigmas der naturwissenschaftlichen Forschung lesen, in dem die Beherrschung und Beseitigung der eigenen Affekte im Dienste interesseloser Erkenntnis (oder Objektivität) einen wesentlichen Teil des wissenschaftlichen Ethos ausmacht. Hier fallen antike Affektlehre und die Selbstdisziplinierung als Figur der modernen Forschung im Rahmen der Selbsttechniken der Optimierung, wie sie für die Gegenwart kennzeichnend sind, zusammen. John vertritt dagegen ein neues Ethos der Tierforschung, das sich der Nutzung und dem Wert der Affektivität hin öffnet, jedoch die Verantwortung für das Andere in den Vordergrund rückt. Marina allerdings ist jene Figur, die am deutlichsten unter dem Affektregime einer Gegenwart steht, in dem Emotionalität zur individuellen Ressource erhoben (vgl. Abschn. 4.2.2.1) und Selbstoptimierung als andauernder Anpassungsprozess im Dienste der Arbeitsmarktfähigkeit betrachtet wird. ${ }^{357}$

Dass Suleiman die Positionen der modernen wissenschaftlichen Objektivität und des postmodernen empathischen Ethos in einem Roman zwei männlichen Figuren und jene des überforderten neoliberalen Selbst ${ }^{358}$ einer Figur aneignet, die sich in diesem Roman gerade daran abarbeitet, eine Frau im wissenschaftlichen Betrieb zu sein, verweist auf eine weitere Schicht der Verhandlung eines emotionalen Ethos in der Wissenschaft: Wer sich nicht vordergründig mit der Selbstbehauptung als Unterprivilegierte im wissenschaftlichen Betrieb und mit der Relevanz geschlechtlicher Kodierungen von Emotionalität für das eigene Fühlen und Handeln auseinandersetzt, gelangt vielleicht rascher zu dem von Griffin propagierten Gleichmut in der Forschungspraxis oder Johns selbstbewusster Argumentation für einen empathischen Umgang mit dem Tier. Der Roman Auswilderung macht auch dieses strukturell angelegte Ungleichgewicht, das für die (Feld-)Primatologie seit ihren Anfängen mit den sogenannten ,Trimates' relevant ist, zum Thema seiner Auseinandersetzung mit Ethiken der Emotionalität in der Forschung(spraxis).

\footnotetext{
${ }^{356}$ Daston/Galison: Objectivity, S. 39. Daston/Galison folgen damit Foucaults Verständnis von Subjektivität als ,nichts Anderes als der Name für das, was sich als Gegenstand eines spezifischen Ensembles von Wissen, Normen und Selbstpraktiken ergibt“. Saar: Die Form des Lebens, S. 329.

${ }^{357} \mathrm{Vgl}$. Mayer/Thompson: Inszenierung und Optimierung des Selbst, S. 7 f.

${ }^{358}$ Vgl. zu dieser Deutungsmöglichkeit Ehrenberg: Das erschöpfte Selbst, S. 219 ff.
} 
Mit dieser Beschäftigung mit Ethiken der Emotionalität und ihrer Rolle in der Forschungspraxis partizipieren die beiden Romane an einem Trend der Gegenwartsliteratur. Literatur sei, so zitiert Stephanie Waldow in ihrer Studie zum Schreiben als Begegnung mit dem Anderen Paul Ricœur, das ,erste Laboratorium des moralischen Urteils“"359 und daher ohnehin immer ethisch und niemals neutral. In der Gegenwartsliteratur im engeren Sinn trete dieser Zug aber - etwa im Vergleich zur postmodernen Literatur in der zweiten Hälfte des 20. Jahrhunderts - wieder deutlicher in den Vordergrund:

Verstärkt werden in der Literatur [nach 1989] Fragen nach einem ,sinnerfüllten Leben aufgeworfen, dies aber nicht mehr im Sinne einer für alle verbindlichen moralisch-normativen Handlungsanweisung oder teleologischen Ausrichtung, sondern in Form eines ethischen Aushandelns. Gefordert wird also eine Orientierung jenseits normativer Setzungen im Sinne eines ethisch-dynamischen Dialogs mit Alteritäten. ${ }^{360}$

Sieben Sprünge vom Rand der Welt und Auswilderung arbeiten an einer in diesem Sinne ,ethischen Wende‘. Denn in den beiden Romanen stehen nicht so sehr die Wissensbestände der Primatologie, die Gefühlsqualität und ihr epistemischer Wert oder der Interspezies-Kontakts im Vordergrund, wie dies bei Peter Goldsworthys Wish und William Boyds Brazzaville Beach der Fall war. Durch ihre hinterfragenden Perspektiven auf die auch in der vorliegenden Studie diskutierten affektiv bedingten Produktionsbedingungen von Wissen über Affen (und in der genetischen und evolutionären Konsequenz auch über Menschen) in der Primatologie regen beide Texte stattdessen zu einer Auseinandersetzung mit Ethiken der Emotionalität als Ethiken der Forschung an. Sie wollen dabei keine literarischen Leitfäden im Sinne einer normativen Ethik anbieten, sondern auf grundlegende Strukturen - historisch und kulturelle bedingte Affektregime, individuelle Persönlichkeitsprofile, institutionelle Gefühlskulturen - hinweisen und diese ganz wörtlich zur Sprache bringen, um Alternativen zur Diskussion zu stellen.

Waldow sieht die ,ethische Wende“ der Gegenwartsliteratur seit 1989 durch im weitesten Sinn zeitgenössische philosophische Überlegungen geschult, deren Ausgangspunkt das Anliegen darstellt,

auf gängige Herrschafts- und Machtstrukturen hinzuweisen und diese dann in einem zweiten Schritt subversiv zu durchbrechen. Gefordert und erprobt wird ein neuer Umgang mit Diskursmustern. Wurde das Subjekt ehemals noch vom herrschenden Diskurs beschrieben, erkennt es nun die Notwendigkeit an, sich im Diskurs selbst zu positionieren. Es ist aber gerade nicht das souveräne Subjekt, welches eine Renaissance erfährt und nun Haltung einnimmt. Voraussetzung für die Notwendigkeit einer Selbstpositionierung ist die Einsicht in die Grenzen und Unvollkommenheiten des eigenen Daseins. ${ }^{361}$

\footnotetext{
${ }^{359}$ Waldow: Schreiben als Begegnung, S. 16; Ricœur, Paul: Das Selbst als ein Anderer. München 1996 (frz. 1990), S. 200.

${ }^{360}$ Waldow: Schreiben als Begegnung, S. 18.

${ }^{361}$ Waldow: Schreiben als Begegnung, S. 198.
} 
Besonders mit Suleimans Protagonistin scheint ein solches unsouveränes literarische Subjekt auf, das ,sich aufgrund der Einsicht in die eigene Unzulänglichkeit und die Grenzen des Wissbaren nicht mehr länger in der Lage [sieht], moralische Entscheidungen zu treffen und normative Wertmaßstäbe zu installieren. "362 Auch Simone Grolmann in Draesners Sieben Sprünge lässt sich als diese Art neues literarisches Subjekt lesen, ist doch ihr Nachdenken über Gefühle ein Versuch einer neuen Selbstpositionierung. Das „,scheinbare ,ethische Scheitern“ wird nun zum Ausgangspunkt ethischen Handelns“, ${ }^{363}$ wie sich an der forschungspraktischen Wende der beiden primatologischen Figuren in Sieben Sprünge zeigt.

Diesem dem Scheitern produktiv offenen Subjekt der Literatur korrespondiert, dass sich die Autor/innen auch selbst offenbaren, sich wieder in einem ethischen Diskurs jenseits des ,Tods des Autors“ positionieren und Verantwortung auch für ihre poetologischen Verfahren übernehmen. Dies äußert sich häufig in den Authentizitätsverweisen der literarischen Texte: ${ }^{364}$ So ist Bettina Suleiman promovierte Philosophin und mit den strukturellen Anforderungen und Hierarchien des akademischen Betriebs vertraut. Auch wenn die Autorin Auswilderung nicht als Schlüsselroman des Max-Planck-Instituts in Leipzig gelesen wissen möchte, bietet sie mit dem extratextuellen Hinweis auf ihre eingehende Recherche vor Ort und mit den intratextuellen Verortungen doch den Hinweis darauf, dass die detaillierte Abbildung eines akademischen Instituts, seiner Arbeitswelt und die affektive Konditionierung einer Wissenschaftlerin ein reales Korrelat hat. ${ }^{365}$ Draesner dagegen ist selbst Kind eines Kriegskindes. Teile der Fluchtbiographie von Eustachius Grolmann sind, wie verschiedenen Essays Draesners aus dem Jahr 2007 zu entnehmen ist, auch Teil von Draesners eigener Familiengeschichte: „Geflohen war nur Großmutter mit meinem 15-jährigen Vater und seinem 25-jährigen, gehbehinderten Bruder. 1947 trafen sie in Bayern ein, nur mehr zu zweit. [...] Der ältere Bruder, verletzt, entkräftet, bekam eine Lungenentzündung und starb“. ${ }^{366}$ Bis in Daten und Anekdoten hinein ist Sieben Sprünge

\footnotetext{
${ }^{362}$ Waldow: Schreiben als Begegnung, S. 198.

${ }^{363}$ Waldow: Schreiben als Begegnung, S. 198.

${ }^{364} \mathrm{Vgl}$. Waldow: Schreiben als Begegnung, S. 327 ff. Waldow sieht in diesem Zusammenhang einen neuen Authentizitätsbegriff sich etablieren: „Eine Authentizität, die nicht mehr länger nur das tatsächlich Erfahrene gelten lässt [...], sondern ein Erzählen, welches den eigenen Wahrnehmungshorizont zur authentischen Ausgangsbasis des Schreibens werden lässt“ (S. 373).

${ }^{365}$ Persönliche Kommunikation, Seminar-Autor/innengespräch mit Bettina Suleiman und Rolf Lappert an der Universität Bern im HS 2015 (Science/Fiction - Lektüren der Primatologie).

${ }^{366}$ Draesner, Ulrike: Zauber im Zoo. Vier Reden von Herkunft und Literatur. Göttingen 2007, S. 68 .
} 
daher von Draesners eigener Familiengeschichte und der damit verbundenen Affektproblematik inspiriert. ${ }^{367}$

Im Rahmen der auf ihrer Bamberger Poetikvorlesung beruhenden Essays hat Draesner bereits Position bezogen zum Stellenwert der Literatur nicht nur als Resonanzraum von Gesellschaft und Wissensdiskurs. Es geht Draesner vor allem darum, „wie Literatur an unseren kulturellen Konstrukten mitbaut“. 368 Insofern scheint der Gegenwartsliteratur eine weitaus größere Rolle zuzukommen, als nur die herrschenden epistemologischen Auseinandersetzungen mit Emotionalität im Rahmen eines ,Affective Turn' abzubilden. In der Form des Gedankenexperiments bietet sie einen Verhandlungsraum für die Ethik des Emotionalen in der Forschung, aus dem ein formendes Potenzial für den Umgang mit Affekten ergehen kann: Sei es in der Anerkennung ihrer epistemischen Anteile, ihrem Einfluss auf die Repräsentationsformen des Wissens oder ihrer Einbettung in historische, soziokulturelle oder institutionelle Affektregime und Gefühlskulturen. „Wir brauchen Fiktion und Wirklichkeit als etwas, das sich unterscheiden lässt", schreibt Draesner, aber ,nicht Unterscheidung ist der Ausgangspunkt, sondern Ähnlichkeit. [...] Mich interessiert eine sachliche Erzählkunst, kombiniert mit Gefühlen, die das Erzählen zeigt und erzeugt" 369 Sie bezieht damit aus poetologischer Warte Stellung zu ihrer eigenen Literatur ebenso wie zu den Forschungserzählungen der Primatologie.

\footnotetext{
${ }^{367}$ Bspw. beruht die Erinnerung, wie Hannes Grolmann seiner Frau Lilly einen Dackel schenkt und ihn im Ofen versteckt, um sie mit dem Geschenk zu überraschen, auf einer Anekdote von Draesners eigenen Großeltern, siehe Draesner: Zauber im Zoo, S. 63 f. Im Roman beginnt die Flucht von Lilly und ihren Söhnen am 19. Januar 1945 mittags (SB, S. 209), Draesner gibt für den Beginn der Flucht ihrer Großmutter mit ihrem Vater und Onkel den 20. Januar 1945, mittags, an, vgl. Draesner: Zauber im Zoo, S. 69.

${ }^{368}$ Draesner: Zauber im Zoo, S. 60.

${ }^{369}$ Draesner: Zauber im Zoo, S. 105.
} 
Open Access Dieses Kapitel wird unter der Creative Commons Namensnennung 4.0 International Lizenz (http://creativecommons.org/licenses/by/4.0/deed.de) veröffentlicht, welche die Nutzung, Vervielfältigung, Bearbeitung, Verbreitung und Wiedergabe in jeglichem Medium und Format erlaubt, sofern Sie den/die ursprünglichen Autor(en) und die Quelle ordnungsgemäß nennen, einen Link zur Creative Commons Lizenz beifügen und angeben, ob Änderungen vorgenommen wurden.

Die in diesem Kapitel enthaltenen Bilder und sonstiges Drittmaterial unterliegen ebenfalls der genannten Creative Commons Lizenz, sofern sich aus der Abbildungslegende nichts anderes ergibt. Sofern das betreffende Material nicht unter der genannten Creative Commons Lizenz steht und die betreffende Handlung nicht nach gesetzlichen Vorschriften erlaubt ist, ist für die oben aufgeführten Weiterverwendungen des Materials die Einwilligung des jeweiligen Rechteinhabers einzuholen. 